\title{
Digital pre-distortion for multiple antenna transmitters
}

\author{
DISSERTATION \\ zur Erlangung des akademischen Grades \\ Doktor \\ im Doktoratsstudium der \\ Technischen Wissenschaften
}

Eingereicht von:

Padmanabhan Madampu Suryasarman

Angefertigt am:

Institut für Nachrichtentechnik und Hochfrequenzsysteme

Beurteilung:

Univ. Prof. Dr. Andreas Springer (Betreuung)

Univ. Prof. Dr. Roman Maršálek

Linz, 25. Februar 2015 



\section{Declaration}

I hereby declare under oath that the submitted doctoral dissertation has been written solely by me without any outside assistance, information other than provided sources or aids have not been used and those used have been fully documented. The dissertation here present is identical to the electronically transmitted text document.

Place:

Date:

Signature: 


\section{Acknowledgments}

I would like to express my deepest gratitude to my adviser, Prof Andreas Springer for giving me the opportunity to carry out my doctoral studies at Johannes Kepler University Linz. Without his continuous support and guidance, this work would not have been possible.

I want to thank the Austrian Center of Competence in Mechatronics (ACCM) and Danube Mobile Communications Engineering (DMCE) for funding my research.

I express my gratitude to many of the members of the system engineering team at DMCE for introducing me to the world of RF systems and their support and valuable suggestions. I am grateful to DMCE for the experience and knowledge I gained by being a member of their projects, and for the lab facilities for my measurements.

I would like to thank all the members of the doctoral evaluation committee for accepting my request and attending my doctoral thesis defense.

Last, but not the least, I want to thank my colleagues, Mr. Liu Peng, Mr. Markus Hoflehner, Mr.Anas Saudi and Dr. Jan Kietlinsky for their friendship and insightful discussions. 


\section{Abstrakt}

Der Bedarf an immer höheren Datenraten in modernen drahtlosen Kommunikationssystemen hat zu der Verwendung von Modulationsschemata geführt welche spektral effizient sind aber nicht konstante Hüllkurven besitzen. Deshalb sind Linearisierungstechniken für Mobilfunkleistungsverstärker (PA) entscheidend für die effiziente, lineare Verstärkung dieser Signale. Die Digitale Vorverzerrung (DPD) ist eine der kosteneffektiven und flexibelsten Linearisierungstechniken. Moderne drahtlose Kommunikationssysteme verbessern die Spektrale-Effizienz und Ausfallssicherheit durch den Einsatz von Mehrantennen-Systemen (MIMO). An der Sendeseite des Mehrantennen-Systems entsteht ein Übersprechen in den unterschiedlichen am Chip integrierten Sendepfaden welches die Leistungsfähigkeit herkömmlicher Vorverzerrung Methoden nachteilig beeinflusst.

Die Cross-over-Vorverzerrung (CO-DPD) kompensiert gemeinsam das Übersprechen und die Nichtlinearität des Leistungsverstärkers und verbessert damit die Qualität der Linearisierung im Vergleich zur herkömmlichen Vorverzerrung erheblich. Allerdings ist CO-DPD eine problematische Wahl wenn die Anzahl von Übertragungswege groß ist, weil ihr Rechenaufwand quadratisch mit der Anzahl der Übertragungswege steigt. Wir präsentieren daher einen Übersprechunterdrückenden Vorverzerrer (CTCDPD) der das Übersprechen und die Nichtlinearität getrennt kompensiert. Der Schwerpunkt dieser Dissertation ist die Untersuchung der Parameteridentifikation, Linearisierungsleistung und des Rechenaufwands von CO-DPD und CTC-DPD. Wir verwendeten die Methode der kleinsten Fehlerquadrate (LS) zur Parameteridentifikation für CTC-DPD Koeffizienten und verglichen die Linearisierungsleistung und den Rechenaufwand mit der CO-DPD. Die elektromagnetische und kapazitive Kopplung zwischen den einzelnen Sendepfaden ist ein frequenzabhängiger Prozess. Diese Frequenzabhängigkeit kann auf die Linearisierungsleistung bzw. auf die Koeffizienten erhebliche Auswirkung haben. Die CTC-DPD-Struktur kann so erweitert werden dass das frequenzselektive Übersprechen berücksichtigt werden kann, wozu die CO-DPD nicht fähig ist. Wir wenden weiters auch adaptive Algorithmen wie den RLS-Algorithmus und den LMS-Algorithmus zur Identifikation der Entzerrerkoeffizienten an und verglichen die Ergebnisse der CTC-DPD mit den Ergebnissen der CO-DPD. Die Linearisierungsleistung der CTC-DPD ist vergleichbar mit der CODPD, jedoch besitzt die CTC-DPD einen niedrigeren Rechenaufwand. 


\section{Abstract}

The requirement for high data rates in modern wireless communication systems has led to the use of spectrally efficient modulation schemes, which result in nonconstant envelope communication signals. Thus, linearization techniques for nonlinear microwave power amplifiers (PA) are crucial for the efficient, linear amplification of such signals. Digital predistortion (DPD) is one of the most cost effective and flexible ways among all linearization techniques. Modern wireless communication systems employ multiple-input multiple-output (MIMO) technology to improve spectral efficiency and link-reliability. At the transmit side of a MIMO communication system, RF cross-talk which occur before the PAs between the different paths which are integrated on the same chip-set adversely affects the performance of conventional DPD techniques.

A cross-over predistortion (CO-DPD) compensates the cross-talk and non-linearity of the PAs jointly and improves the quality of predistortion significantly compared to the conventional DPD. However, CO-DPD becomes a problematic choice when the number of transmit paths is large because its computational complexity increases quadratically with the number of transmit paths. We present a cross-talk canceling predistorter (CTC-DPD) which compensates the cross-talk and PA non-linearity separately. The focus of this dissertation is the parameter identification, linearization performance and computational complexity of CO-DPD and CTC-DPD. We applied the least squares (LS) method for parameter identification for CTC-DPD coefficients and compared its linearization performance and computational complexity to CO-DPD. The electromagnetic and capacitive coupling between the transmit paths are frequency dependent processes and sometimes its effect can be considerable. The CTC-DPD structure can be extended to pre-cancel frequency selective cross-talk, while CO-DPD can not. We also applied the recursive least squares (RLS) and least mean squared (LMS) adaptive algorithms for CTC-DPD and similarly compared it to adaptive CO-DPD. CTC-DPD is found to be performing equally good as CO-DPD, while it is computationally much simpler. 


\section{Contents}

1. Introduction 1

1.1. Background and motivation . . . . . . . . . . . . . . 1

1.2. Outline of the thesis and Contributions . . . . . . . . . . . . . 4

2. Power amplifier non-idealities and digital predistortion $\quad 6$

2.1. Linearity and efficiency of power amplifiers . . . . . . . . . . . . . 6

2.2. PA non-linearity and memory effects . . . . . . . . . . . . . . 8

2.3. Behavioral modeling of PA characteristics . . . . . . . . . . . . 9

2.4. Digital predistortion . . . . . . . . . . . . . . 12

2.5. Direct and indirect learning architectures . . . . . . . . . . . . 13

3. Digital predistortion for MIMO transmitters $\mathbf{1 5}$

3.1. Power amplifier non-linearity and RF cross-talk in MIMO transmitters 16

3.2. Cross-over and cross-talk canceling predistortion techniques . . . . . 18

3.2.1. Estimation of coupling factors for CTC-DPD . . . . . . . . 23

3.2.2. Frequency selective cross-talk . . . . . . . . . . . . . . 24

3.3. Measurement setup . . . . . . . . . . . . . . . . . 26

3.4. LInearization performance indicators . . . . . . . . . . . . . . 31

3.5. Measurement results and discussion . . . . . . . . . . . . . 35

3.5.1. Frequency flat cross-talk . . . . . . . . . . . . . . 38

3.5.2. Frequency selective cross-talk . . . . . . . . . . . . . . 41

3.6. Measurements with cross-talk using RF components . . . . . . . . . . 43

4. Adaptive predistortion for MIMO transmitters 46

4.1. Adaptive predistortion . . . . . . . . . . . . . . . . . . 47

4.2. Adaptive algorithms for cross-over and cross-talk canceling DPDs . . 49

4.3. Simulation results and discussion . . . . . . . . . . . . . . 51

5. Complexity Analysis $\quad \mathbf{5 9}$

5.1. Complex and real operations . . . . . . . . . . . . . . . . . . 59

5.2. Least squares estimation of DPD . . . . . . . . . . . . . 60

5.3. Estimation of coupling coefficients for CTC-DPD . . . . . . . . . 61

5.4. Memory polynomial DPD . . . . . . . . . . . . . . 62

5.5. Cancelling the cross-talk . . . . . . . . . . . . . . . 62

5.6. Comparing the complexity of CO-DPD and CTC-DPD . . . . . . . 63

5.7. Complexity of adaptive MIMO predistortion . . . . . . . . . . . 66 
$\begin{array}{lll}\text { 6. Conclusions } & 68\end{array}$

$\begin{array}{ll}\text { Appendix A. Measurement results (Two antenna Tx) } & 71\end{array}$

Appendix B. Measurement results (Single antenna Tx) 76

Appendix C. Accuracy of cross-talk estimation $\quad 84$

$\begin{array}{lr}\text { Bibliography } & 89\end{array}$ 


\section{List of Figures}

1.1. Cross-over DPD . . . . . . . . . . . . . . . . . . . . 3

1.2. Cross-talk canceling DPD . . . . . . . . . . . . . 4

2.1. The drain efficiency of an ideal class-A and class-B power amplifier . 7

2.2. Power amplifier response . . . . . . . . . . . . . . . . 8

2.3. Wiener and Hammerstein models . . . . . . . . . . . . . . . . . . . . 11

2.4. Predistortion concept . . . . . . . . . . . . . . . . 12

2.5. Direct and indirect learning architectures . . . . . . . . . . . . . 13

3.1. Indirect learning architecture for predistortion . . . . . . . . . . . . 16

3.2. Conventional predistortion (C-DPD) in the presence of non-linear cross-talk . . . . . . . . . . . . . . . . . 17

3.3. Cross-over digital predistortion (CO-DPD) for 2 antenna transmitter 19

3.4. Cross-talk canceling digital predistortion (CTC-DPD) for 2 antenna

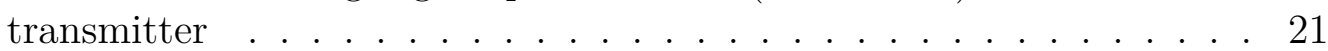

3.5. CO-DPD for 4 antenna Transmitter . . . . . . . . . . . . . . . . . 22

3.6. CTC-DPD for 4 antenna Transmitter . . . . . . . . . . . . . . . 23

3.7. The measurement setup . . . . . . . . . . . . . . . . . 27

3.8. AM-AM plots (signal E) . . . . . . . . . . . . . . . . . . . . . . . . . . . . . . . . 28

3.9. PSD spectra (signal E) . . . . . . . . . . . . . . . . . . . . . . . . . . . . . . . 38

3.10. Empirical PDF of the input signal magnitude . . . . . . . . . . . 30

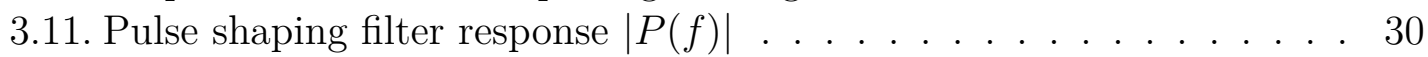

3.12. Residual leakage with CO-DPD . . . . . . . . . . . . . . . . . . . . . . . . 34

3.13. Residual leakage with CTC-DPD . . . . . . . . . . . . . 35

3.14. Comparison of C-DPD, CO-DPD and CTC-DPD, signal E, PEP in $=$

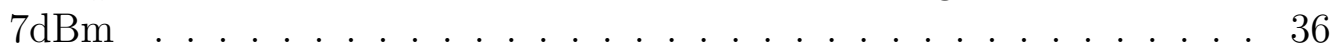

3.15. Measurement results, $\Delta \mathrm{EVM}_{\mathrm{IB}}, \Delta \mathrm{NMSE} \ldots \ldots \ldots \ldots$

3.16. Measurement results (EVM \& ACLR) for signal F, with frequency flat cross-talk . . . . . . . . . . . . . . . 38

3.17. Measurement results (EVM \& ACLR) for signal E with frequency flat cross-talk . . . . . . . . . . . . . . . . . . 39

3.18. Measurement results (EVM \& ACLR) for signal E with frequency selective cross-talk, $\mathrm{PEP}_{\text {in }}=7 \mathrm{dBm}$, using filter 1a from Fig. 3.20 . . 41

3.19. Impulse responses of the coupling filters, $T_{s}=1 / F_{s}$ is the sampling duration , $M=40$ for filter $1, M=100$ for filter 2,3 . . . . . . . . 42 
3.20. Frequency responses of the coupling filters applied to UMTS signals $\left(F_{s}=38.4 \mathrm{MHz}\right) . \ldots \ldots \ldots \ldots$. . . . . . . . . 42

3.21. Measurement setup for MIMO-DPDs, with cross-talk using RF com-

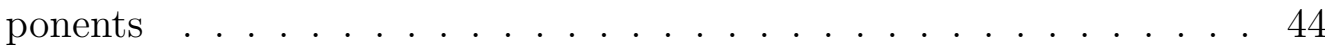

3.22. Measurement results (EVM \& ACLR) for signal $\mathrm{F}$, with frequency flat cross-talk using RF components, PEP of $x_{1}(n)$ and $x_{2}(n)=7 \mathrm{dBm} 45$

4.1. Adaptive transversal filter . . . . . . . . . . . . . . . . . . . . . . . 47

4.2. Comparison of C-DPD and MIMO-DPDs, LMS-DPD, $N_{t}=2$, signal $\mathrm{B}, \mathrm{PA}_{1}$, Frequency flat cross-talk. . . . . . . . . . . . 51

4.3. AM-AM plots (LMS-DPD), Signal D. . . . . . . . . . . . 52

4.4. Spectra (LMS-DPD), Signal D. . . . . . . . . . . . . . 52

4.5. Simulation results, $N_{t}=2$, LMS-DPD, LMS cross-talk estimation. x-axis: $n_{s c}=4\left(n_{s}-1\right)+n_{c}$. $n_{s}$ is 1-7 for signals A-G and $n_{c}$ is $1,2,3,4$ for cross-talk levels $-15 \mathrm{~dB},-20 \mathrm{~dB},-30 \mathrm{~dB}$, and no cross-talk. . . . . . 54

4.6. Filtered error energy $\xi(n), N_{t=2}$, LMS-DPD, signal B, $\mathrm{PA}_{1}$, Frequency flat cross-talk . . . . . . . . . . . . . . . . 56 56

4.7. Frequency responses of the coupling filters . . . . . . . . . . . . 57

4.8. Simulation results, $N_{t}=2$, signal B, $\mathrm{PA}_{1},-20 \mathrm{~dB}$ frequency selective cross-talk . . . . . . . . . . . . . . . 57

A.1. Measurement results, $\triangle \mathrm{NMSE}_{\mathrm{IB}}, \triangle \mathrm{EVM}, \triangle \mathrm{ACLR}$ and $\triangle \mathrm{ACEPR}$. . 73

A.2. Measurement results, NMSE $\mathrm{IB}_{\mathrm{B}}, \mathrm{EVM}, \mathrm{ACLR}$ and ACEPR . . . . . . . 74

A.3. Measurement results, $\varepsilon_{L S}, \varepsilon_{\text {Leak }}, \mathrm{P}_{\text {out }}$ and $\mathrm{PEP}_{\text {out }} \ldots \ldots . . . .75$

B.1. PA output power . . . . . . . . . . . . . . 76

B.2. AM-AM plots (signal E), $k=1,6 \ldots \ldots \ldots 78$

B.3. PSD spectra $($ signal E), $k=1,6 \ldots \ldots \ldots \ldots$

B.4. AM-PM plots (signal E) . . . . . . . . . . . . . . . . . 79

B.5. $\varepsilon_{\mathrm{amp}} \ldots \ldots \ldots \ldots \ldots \ldots \ldots$

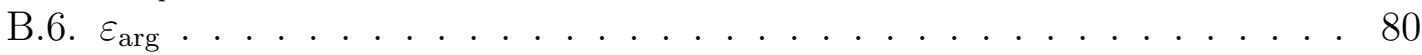

B.7. Constellation diagrams, $\mathrm{PEP}_{\mathrm{in}}=7 \mathrm{dBm} \ldots \ldots \ldots . . \ldots 81$

B.8. Constellation diagrams, $\mathrm{PEP}_{\text {in }}=10 \mathrm{dBm}$. . . . . . . . . . . . 82

B.9. Set 1: EVM \& ACLR . . . . . . . . . . . . . . . . . . . . 83

B.10.Set 2: EVM \& ACLR . . . . . . . . . . . . . . . . . 83

C.1. Average error in cross-talk estimation $\left(\varepsilon_{\alpha}\right)$ in block based cross-talk estimation, signal $\mathrm{G}, k$ is the block number. . . . . . . . . 85

C.2. Average error in cross-talk estimation $\left(\varepsilon_{\alpha}\right)$ in sample-by-sample crosstalk estimation (LMS), signal G . . . . . . . . . . . . . . 85

C.3. Average error in cross-talk estimation $\left(\varepsilon_{\alpha}\right)$ from the simulations as described in (C.4), signal G, $\mathrm{PEP}_{\text {in }}=7 \mathrm{dBm},-20 \mathrm{~dB}$ cross-talk, $k$ is the block number. 


\section{List of Tables}

3.1. Details of the measurements . . . . . . . . . . . . . . . . . 29

3.2. Details of the Transmit signals . . . . . . . . . . . . . . . . . 32

3.3. Details of UMTS signals . . . . . . . . . . . . . . . . . 32

3.4. Measurement results for signal E \& F . . . . . . . . . . . . . . . . . . 40

3.5. Measurement results for signal $\mathrm{E}, \mathrm{PEP}_{\text {in }}=7 \mathrm{dBm},-20 \mathrm{~dB}$, Frequency selective cross-talk . . . . . . . . . . . . . . 43

4.1. Details of the simulation setup . . . . . . . . . . . . . . 53

4.2. Comparison of cross-talk estimation methods, $N_{t}=2,-20 \mathrm{~dB}$, Fre-

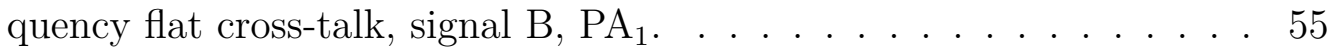

4.3. Simulation results, $N_{t}=4$, frequency flat cross-talk, signal $\mathrm{B}, \mathrm{PA}_{1}$, LMS-DPD, LMS cross-talk estimation . . . . . . . . . . . . 58

5.1. Comparison of complexity, $N_{t}=2, K=9, Q=3, M=1 \ldots$. . . 63

5.2. Complexity ratio (\%), $N_{t}=2, N=10^{3} \ldots \ldots \ldots$. . . . . 64

5.3. Complexity ratio $\eta[\%], M=1, Q=3, N=10^{3} \ldots \ldots$. . . . . . 64

5.4. Comparison of complexity of adaptive MIMO predistortion, $K=9$,

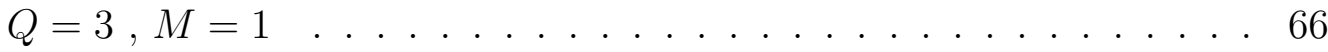

5.5. Complexity ratio $\eta[\%]$, adaptive MIMO predistortion, $N_{t}=2 \ldots$. . 66

5.6. Complexity Ratio $\eta[\%]$, adaptive MIMO predistortion, $M=1, Q=367$

B.1. PA output power $[\mathrm{dBm}] \ldots \ldots \ldots \ldots$. . . . . . . . . . . . 77 



\section{Chapter 1.}

\section{Introduction}

\subsection{Background and motivation}

The tremendous growth in the demand for wireless connectivity in recent years has caused an increasing need for high data rates in new wireless applications. Modern wireless communication systems achieve high data rates by employing multipleinput-multiple-output (MIMO) technology and advanced modulation schemes. The use of multiple antennas at transmitter and receiver, popularly known as MIMO, has attracted a great deal of attention in wireless communications, because it offers significant increases in data throughput and link range without additional bandwidth or increased transmit power. The performance improvements resulting from the use of MIMO systems are due to array gain, diversity gain, spatial multiplexing gain, and interference reduction [1]. Array gain is the increase in average receive SNR achieved through the use of multiple antennas at the receiver and/or transmitter. If the $N_{t} \times N_{r}$ links ${ }^{1}$ composing the MIMO channel fade independently and the transmitted signal is suitably constructed, the receiver can combine the arriving signals to achieve $N_{t} \times N_{r}$ th-order diversity. Spatial multiplexing gain refers to the linear increase in capacity when the MIMO channels are used to transmit multiple (up to $\min \left(N_{t}, N_{r}\right)$ ) independent data streams. Under favorable channel conditions, such as rich scattering, the receiver can separate the different streams. Multiple antennas at the transmitter/receiver can also be used to reduce the co-channel interference arising from the frequency reuse in wireless channels.

The requirements for both high data rates and efficient utilization of the increasingly crowded spectrum leads to the use of shaped data pulses (e.g. root raised cosine pulse shaping), higher order and spectrally efficient modulation schemes (e.g. 16-QAM, 64-QAM, OFDM) and advanced multiple access schemes such as CDMA. To permit multiple access without undue interference between the users, CDMA employs spread-spectrum technology. CDMA is used as the access method in the $3^{\text {rd }}$ generation (3G) cellular standards such as CDMA2000 and WCDMA. The $4^{\text {th }}$ generation $(4 \mathrm{G})$ cellular standards such as LTE and LTE-Advanced employ OFDM, which is a spectrally efficient multi-carrier modulation method. OFDM can adapt to severe

\footnotetext{
${ }^{1} N_{t}$ and $N_{r}$ are the number of transmit and receive antennas, respectively.
} 
channel conditions without complex time domain equalization, as it can perform simple and efficient single-tap frequency domain channel equalization. OFDMAthe multi-user version of OFDM - can efficiently utilize the multi-user diversity in time and frequency domains by opportunistic user scheduling, and thereby achieves a very high system throughput. LTE and LTE-Advanced can achieve maximum link-spectral efficiencies of 16 and $30 \mathrm{bits} / \mathrm{s} / \mathrm{Hz}$ with the use of $4 \mathrm{x} 4$ and $8 \times 8$ MIMO respectively [2]. While such technologies are successful in achieving high spectral efficiencies, they pose new challenges to multi-antenna transmitter design.

One of the main drawbacks of the sophisticated non-constant envelope modulation schemes such as WCDMA and OFDM is that they have a very high Peak-to-AveragePower-Ratio (PAPR) often exceeding $10 \mathrm{~dB}$ [3]. This makes the design of the power amplifier (PA) and the liniearization techniques tougher. The PA - which is usually the major power-consuming block in wireless devices - must operate with high power efficiency and meet strict linearity requirements to avoid spectrum re-growth, especially when the transmit power is high. Power amplifiers typically operate most efficiently at or near the saturation point. However, the response of the power amplifier at or near saturation point is non-linear. Therefore, there is a trade-off between efficiency and linearity. Digital predistortion (DPD) is one of the widely used techniques to improve the PA linearity while keeping its efficiency, by digitally applying an inverse of the PA response on the input signal to compensate for the distortion introduced by the power amplifier.

At the transmit side of a MIMO communication system, the PA non-linearity and the RF cross-talk between the different paths which are integrated on the same chip-set are the two major sources of impairments [4-7]. Crosstalk can be classified as linear or nonlinear. It is considered linear when the effect of the crosstalk at the output of the transmitter can be modeled as a linear combination of the desired and interference signals. The antenna crosstalk, which is the leakage of the transmitted signal from one antenna element to another antenna element, can be modeled as linear cross-talk. The compensation of linear cross-talk is usually done at the receiver along with combating the effects of the wireless channel and receiver cross-talk. On the other hand, the effect of the cross-talk which takes place before the PA is nonlinear, due to the non-linear behavior of the PA [8]. In the presence of non-linear cross-talk in MIMO transmitters, the performance of the conventional DPD (CDPD) algorithms degrades considerably. The focus of this thesis is thus put on the deleterious effect of non-linear cross-talk on predistortion in MIMO transmitters, and the methods proposed to circumvent it. We do not consider the linear crosstalk, as it has no effect on predistortion.

A cross-over predistortion (CO-DPD) as introduced in [8] and shown in Fig. 1.1, compensates the cross-talk and non-linearity of the PAs and significantly improves the quality of predistortion compared to C-DPD. However, the structure of the CO-DPD can become complex as the number of transmit paths increases. The CODPD becomes impractical in situations where the number of transmit paths becomes large because the complexity of the digital predistorter increases quadratically with 


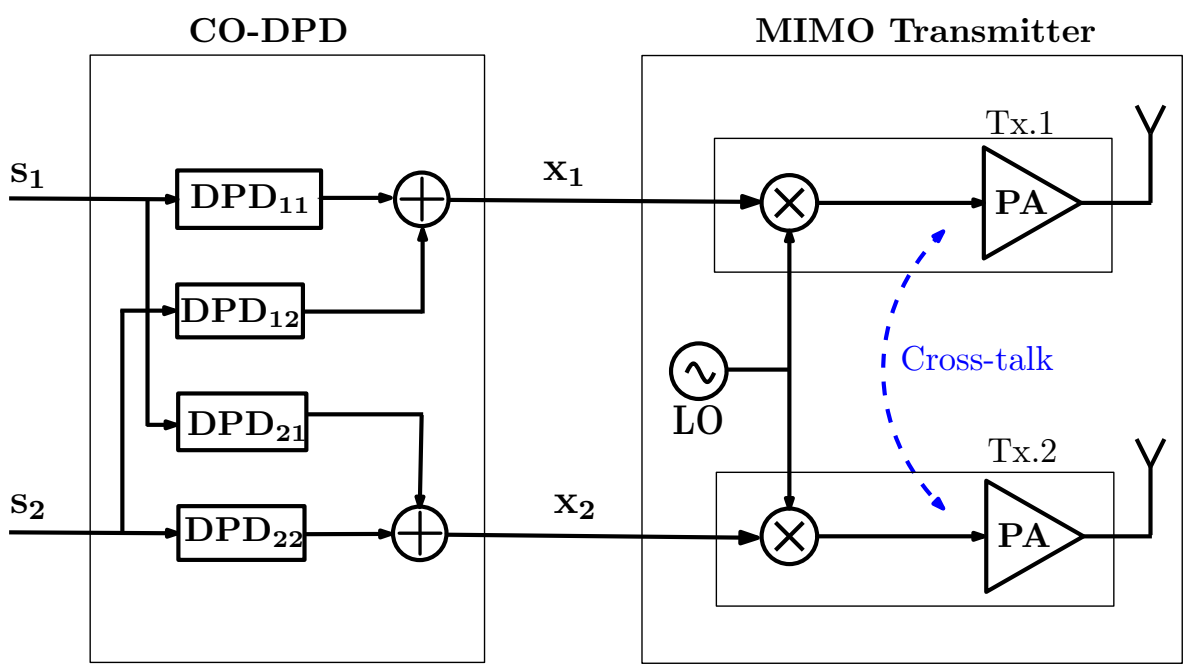

Fig. 1.1: Cross-over DPD

the number of RF transmit paths. Moreover, as the cross-over model for MIMO transmitters ignores the higher order cross-terms between the input signals, the linearization performance of CO-DPD degrades, especially at high cross-talk levels.

In [9], we proposed a cross-talk canceling predistorter (CTC-DPD) which accurately models and compensates cross-talk and non-linearity. In the CTC-DPD structure, the DPDs are followed by a block which pre-cancels the cross-talk that occurs before the PAs, as shown in Fig. 1.2. The estimation of the coupling factors - by which we model the cross-talk - for CTC-DPD is discussed in [9], [10] and [11]. The leakage between the transmitters is sometimes frequency selective, and its effect can be considerable in the case of broad-band transmit signals. The frequency selective coupling can be modeled using a finite impulse response (FIR) filter and the CTCDPD structure can be extended to pre-cancel it. The main properties of CO-DPD and CTC-DPD can be summarized as

- CO-DPD jointly compensates the RF cross-talk and the PA nonlinearity.

- In CTC-DPD, cross-talk cancellation and PA linearization are decoupled. The RF cross-talk occurs before the PA in the transmit path, so the cross-talk cancellation is done after the predistortion.

- The cross-over model ignores the higher order cross-terms between the input signals, affecting the performance of CO-DPD at high cross-talk levels. CTCDPD does not have this problem.

- CO-DPD does not require the knowledge of the coupling coefficients.

- The number of branch predistorters required, is equal to the the square of the number of RF transmit paths for CO-DPD and is equal to the number 


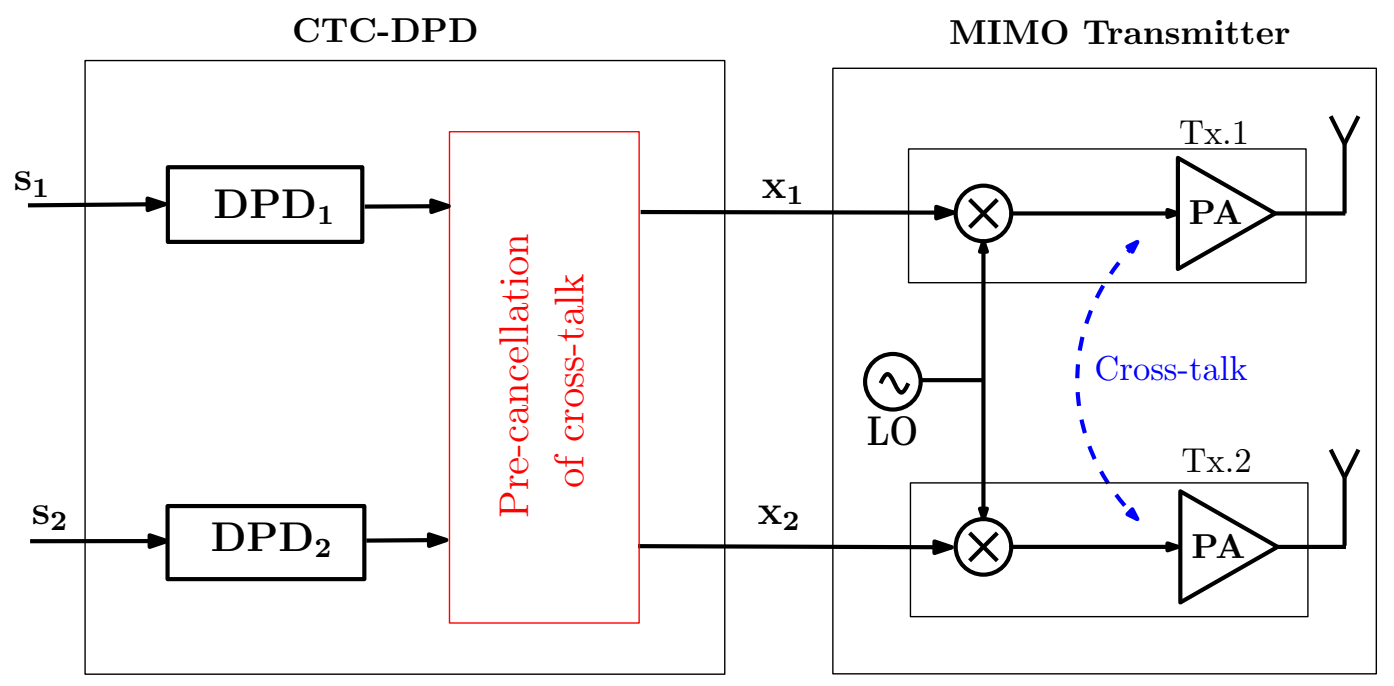

Fig. 1.2: Cross-talk canceling DPD

of RF transmit paths for CTC-DPD. This makes CTC-DPD computationally simpler than CO-DPD.

- CTC-DPD can be extended to deal with the frequency selectivity of the crosstalk, but CO-DPD can not.

\subsection{Outline of the thesis and Contributions}

In this section, we present the organization and the main contributions of the thesis.

Chapter 2 explains the importance of the PA linearization in wireless transmitters. Firstly, the trade-off between linearity and efficiency in PAs is discussed. Terms such as $1 \mathrm{~dB}$ compression point, third order intercept point, drain efficiency, power added efficiency and overall efficiency, which are important in PA design and linearization are explained. The non-linear distortions introduced by the PA (AM-AM, AMPM conversions, memory effects) and several Volterra-based behavioral models of power amplifiers such as Wiener-, Hammerstein- and memory polynomial models are also described in this chapter. Finally, the methods of PA linearization (such as feedforward, feedback and predistortion) and the direct and indirect learning architectures for predistortion are discussed.

Chapter 3 presents a detailed description of the indirect learning architectures, parameter identification and linearization performance of MIMO-DPDs (i.e., CO-DPD and CTC-DPD) and C-DPD for a two antenna transmitter. Firstly, the least squares (LS) estimation of the DPD coefficients for a single antenna transmitter is explained, This method can be extended for CO-DPD [8] and CTC-DPD, which we proposed 
in [9]. We show that the CO-DPD coefficients extracted using the indirect learning architecture form an accurate post-distorter for a MIMO transmitter, but not an accurate predistorter. For the construction of CTC-DPD, the coupling factors which model the cross-talk need to be estimated. We describe a method to estimate the coupling factors using the linear section of the PA response [10]. The extension of CTC-DPD for frequency selective cross-talk which is described in this chapter was also introduced in [10]. Finally, the results of measurements with two different PA responses and transmit signals such as QPSK, 16-QAM, WCDMA (used in UMTS uplink) and SC-FDM (used in LTE uplink) for a two antenna transmitter are presented.

Chapter 4 is about the applicability of adaptive algorithms (LMS and RLS) for the MIMO-DPDs. Firstly, the conventional adaptive predistortion - where the DPD coefficients are updated every sample duration - for a single antenna transmitter is explained. These algorithms can be extended for MIMO-DPDs. In the case of CTC-DPD, the coupling factors are also adaptively estimated along with the DPD coefficients. We proposed the adaptive algorithms for CTC-DPD in [12], and presented a detailed analysis of adaptive CTC-DPD and CO-DPD in [13]. The adaptive MIMO-DPD algorithms are evaluated based on simulations. PA models which are extracted from the measurements (chapter 3) and the same set of transmit signals used in the measurements, are used in the simulations.

Chapter 5 compares the computational complexities of CTC-DPD and CO-DPD. CTC-DPD is computationally simpler compared to that of CO-DPD, as it has only $N_{t}$ predistorter branches ( $N_{t}$ is the number of transmit paths) and a cross-talk precancellation block, whereas CO-DPD has $N_{t}^{2}$ predistorter branches. 


\section{Chapter 2.}

\section{Power amplifier non-idealities and digital predistortion}

Efficient, linear amplification of RF signals with non-constant envelope is one of the major design challenges in modern wireless transceiver design. Digital predistortion is one of the most popular and cost-effective techniques used to achieve this goal. This chapter discusses the trade-off between linearity and efficiency in power amplifiers, the non-linear distortions and the behavioral modeling of power amplifiers and the techniques to improve the linearity, especially digital predistortion.

\subsection{Linearity and efficiency of power amplifiers}

Amplification of information bearing signals is an integral part of every wireless transmitter. The perfectly linear ideal memoryless amplifier produces an output signal that is a multiple of its input signal. In reality, no amplifier is able to provide linear gain for all possible signal power levels. Practical amplifiers generally exhibit non-linearity and memory effects. The need for linearity is one of the principal drivers in the design of modern power amplifiers (PA). In wireless communication systems, an essential constraint implies that communication in other frequency bands must not be disturbed excessively, while the transmitter boosts the signal to a sufficient power level in order to supply the receiver with a sufficiently high level of signal power. The distortions caused by the power amplifier include amplitude nonlinearity, amplitude-to-phase conversion and memory effects. The result is unwanted leakage of signal energy into adjacent channels and distortion of the signal within the transmission bandwidth.

The requirements for both high data rates and efficient utilization of the increasingly crowded spectrum necessitates the use of shaped data pulses (e.g. root raised cosine pulse shaping) and higher order and spectrally efficient modulation schemes (e.g. 16-QAM, 64-QAM, OFDM). The improved spectral efficiency comes at the expense of an increased Peak-to-Average-Power-Ratio (PAPR) of the time domain signal. Signals such as CW, FM, classical FSK, and GMSK have constant envelopes and therefore do not require linear amplification. In contrast, signals with both 


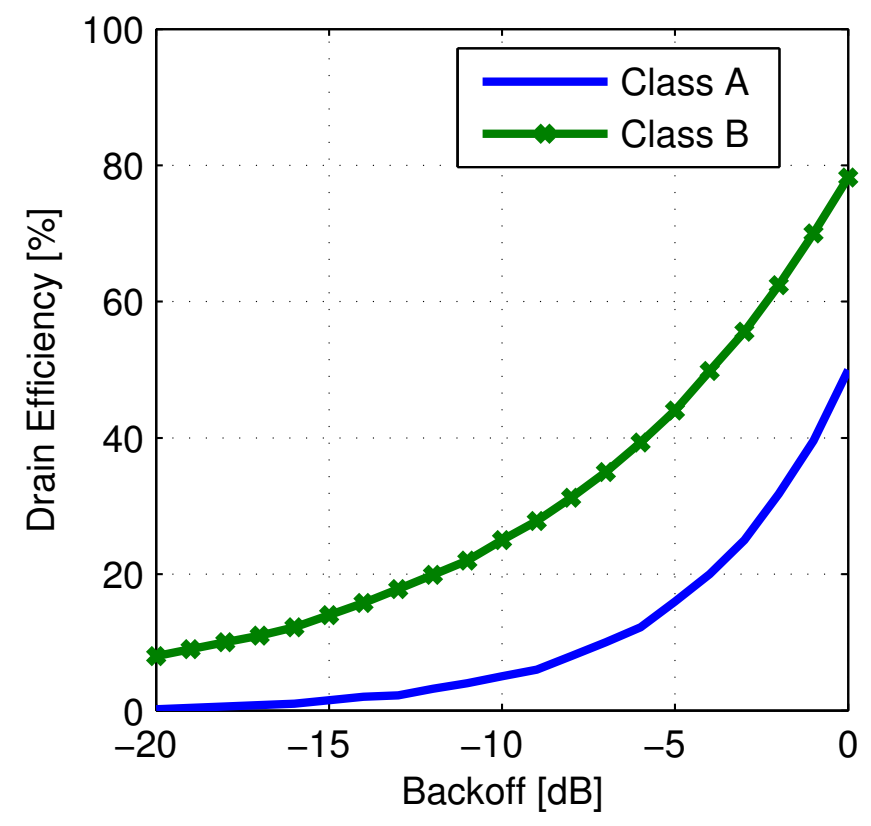

Fig. 2.1: The drain efficiency of an ideal class-A and class-B power amplifier

amplitude and phase modulation (e.g. SSB, vestigial-sideband, digital modulation formats like QAM, QPSK and CDMA and OFDM signals) have non-constant envelopes (and thus high PAPR) and require a highly linear PA.

Efficient conversion of supplied power into radiated signal power is a key requirement in wireless communications. The energy efficiency of mobile handsets heavily depends on the PA efficiency because the PA is usually the major power-consuming block, despite an excessive and increasingly complex digital circuitry $[14,15]$. So, improving the PA efficiency is important in increasing the battery life of the mobile phones. PA efficiency in the base station results in lower operating costs due to a reduced energy supply and smaller cooling units [16].

In PA design, three definitions of efficiency are generally used [17]. The first one is the drain efficiency defined as $\eta_{D}=P_{O} / P_{\mathrm{DC}}$, where $P_{O}$ is the RF output power and $P_{\mathrm{DC}}$ is the total power supplied by the power supply. As the drain efficiency does not take the PA input power in to account, it can over-estimate the PA efficiency, when the PA is operated with a low gain. So, the power added efficiency is defined as $\eta_{\mathrm{PAE}}=\left(P_{O}-P_{i}\right) / P_{\mathrm{DC}}$, where $P_{i}$ is the $\mathrm{RF}$ input power to the $\mathrm{PA}$. An overall efficiency $\eta_{D}=P_{O} /\left(P_{\mathrm{DC}}+P_{i}\right)$ can also be defined. These three expressions are the instantaneous efficiencies at one specific output level, and exactly characterize the efficiency in the case of constant-envelope signals at PA input. For non-constantenvelope signals, the time-averaged efficiency can be evaluated by integrating the product of the instantaneous efficiency and the PDF of the envelope over the range of the envelope. For most PAs, the instantaneous efficiency is highest at the peak output power and decreases as output decreases. The drain efficiency of class A and 


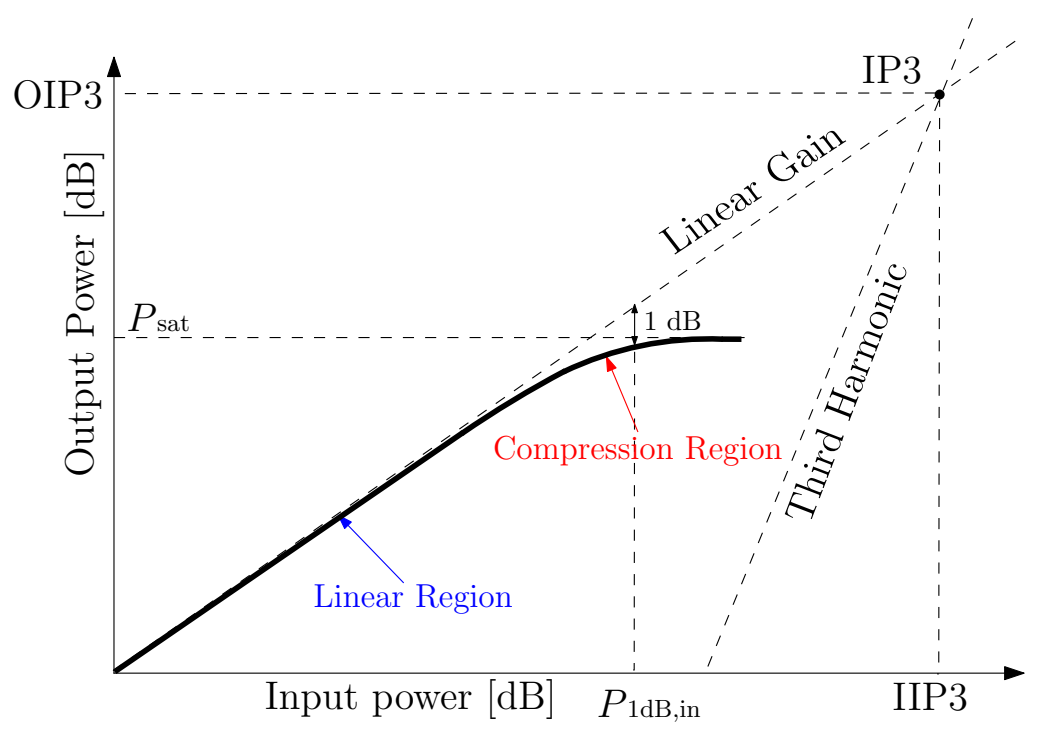

Fig. 2.2: Power amplifier response

B PAs are shown in Fig. 2.1. The amplification of non-constant envelope signals are usually carried out by the linear class (A, AB and B) of PAs (The term 'linear amplifier' does not mean generally that such an amplifier is perfectly linear). If the PA is operated with a large back-off from its saturated output power $\left(P_{\text {sat }}\right)$ to maintain linear amplification, the average power efficiency is severely compromised [18]. On the other hand, if the PA is operated close to the compression region to obtain high power efficiency, it results in non-linear distortions in the output signal. Several techniques which are used to improve the linearity of the PA, without compromising much on its power efficiency are briefly described in section 2.4.

\subsection{PA non-linearity and memory effects}

As the input power is increased above the linear region where the amplifier gain is constant, the PA enters in to the compression region where the gain drops and the output power does not increase linearly with the input power anymore. This is illustrated in Fig. 2.2. The 1- $\mathrm{dB}$ compression point $\left(P_{1 \mathrm{~dB} \text {,in }}\right)$ indicates the input power level that causes the gain to drop by $1 \mathrm{~dB}$ from its small signal value. The 1$\mathrm{dB}$ compression point is a good measure of the linear range of the PA. Now, consider a single tone voltage signal $v_{i}(t)=A \cos \left(\omega_{1} t\right)$ at the input of a memoryless PA. The output signal can be represented as

$$
v_{o}(t)=\sum_{n=1}^{\infty} \alpha_{n} v_{i}^{n}(t)
$$


This can be expressed as a Fourier series [19]

$$
v_{o}(t)=0.5 a_{0}(A)+\sum_{n=1}^{\infty} a_{n}(A) \cos \left(n \omega_{1} t\right)+\sum_{n=1}^{\infty} b_{n}(A) \sin \left(n \omega_{1} t\right)
$$

The component at frequency $\omega_{1}$ is

$$
v_{o 1}(t)=a_{1}(A) \cos \left(\omega_{1} t\right)+b_{1}(A) \sin \left(\omega_{1} t\right)=F_{A}(A) \cos \left(\omega_{1} t+F_{P}(A)\right)
$$

For a modulated narrow-band signal $v_{i}(t)=A(t) \cos \left(\omega_{1} t+\phi(t)\right)$ at the PA input, the output component around the frequency $\omega_{1}$ is

$$
v_{o 1}(t)=F_{A}(A(t)) \cos \left(\omega_{1} t+\phi(t)+F_{P}(A(t))\right)
$$

where $F_{A}(A(t))$ and $F_{P}(A(t))$ are the AM/AM and AM/PM characteristics, respectively. Now consider a two tone signal at the input of the PA

$$
v_{i}(t)=A\left(\cos \left(\omega_{1} t\right)+\cos \left(\omega_{2} t+\phi\right)\right)
$$

The two types of distortions produced due to the non-linear behavior of the PA are harmonics and intermodulation products. The harmonic distortion produces frequency components that are multiples of the fundamental frequencies $\omega_{1}$ and $\omega_{2}$, and their influence to communication systems can be easily removed by filtering. The intermodulation products create frequency components at $2 \omega_{1}-\omega_{2}, 2 \omega_{1}+\omega_{2}$, $2 \omega_{2}-\omega_{1}, 2 \omega_{2}+\omega_{1}, 3 \omega_{1}-\omega_{2}$ etc. The intermodulation products from all the even order non-linear terms fall out-of-band and are not harmful. Some of the odd order intermodulation products fall in to the frequency band of the input signal. The intercept point of third order, IP3 is a good indicator of the linearity of the PA as the strongest in-band intermodulation components are usually created from the $3^{\text {rd }}$ order non-linearity $\left(2 \omega_{1}-\omega_{2}\right.$ and $\left.2 \omega_{2}-\omega_{1}\right)$. IP3, as shown in Fig. 2.2 , is the intersection of the linear and $3^{\text {rd }}$ order components. IIP3 and OIP3 represent the input and output IP3 points respectively.

As the signal bandwidth increases, power amplifiers used in wideband telecommunication systems exhibit memory effects, i.e. frequency dependence, besides nonlinearities. Asymmetries between upper and lower intermodulation products and spectral regrowth are attributed to PA memory effects [20,21]. RF PAs are affected by short-term and long-term memories. Short-term memory is primarily caused by the frequency response of matching networks, filter group delays and transit time within the transistors. Long-term memory is due to biasing circuits, automatic gain control circuits, transistor self-heating and trapping effects $[22,23]$.

\subsection{Behavioral modeling of PA characteristics}

Power amplifier models can be classified as physical models and behavioral models [16,24]. Physical models [25] are the ones requiring knowledge of the electronic 
elements that comprise the PA, their constitutive relations, and the theoretical rules describing their interactions. When no a-priori knowledge of the internal composition is available, power amplifiers are usually modeled as parametric black boxes which rely exclusively on a set of wisely selected input-output observations. The parameters do not reflect physical entities in the system. They are commonly referred as behavioral models. The volterra based behavior models which are capable of modeling a very large class of non-linear systems are discussed in this section. The equivalent baseband volterra series of order $2 P-1$, which model the dynamic non-linear behavior of the PA can be written as $[16,26-28]$

$$
y(t)=\sum_{p=0}^{P-1} \int \ldots \int h_{2 p+1}\left(\boldsymbol{\tau}_{2 p+1}\right) \prod_{i=1}^{p+1} x\left(t-\tau_{i}\right) \prod_{i=p+2}^{2 p+1} x^{*}\left(t-\tau_{i}\right) d \boldsymbol{\tau}_{2 p+1},
$$

$x(t)$ and $y(t)$ are the complex baseband input and output signals. $h_{2 p+1}\left(\boldsymbol{\tau}_{2 p+1}\right)=$ $h_{2 p+1}\left(\tau_{1}, \tau_{2}, \ldots, \tau_{2 p+1}\right)$ is the $2 p+1$ dimensional basband volterra kernel, and it is related to the passband kernel $\tilde{h}_{2 p+1}\left(\boldsymbol{\tau}_{2 p+1}\right)$ as

$$
h_{2 p+1}\left(\boldsymbol{\tau}_{2 p+1}\right)=\left(\frac{1}{2}\right)^{2 p}\left(\begin{array}{c}
2 p+1 \\
p
\end{array}\right) \tilde{h}_{2 p+1}\left(\boldsymbol{\tau}_{2 p+1}\right) e^{-j 2 \pi f_{0}\left(\sum_{i=1}^{p+1} \tau_{i}-\sum_{i=p+2}^{2 p+1} \tau_{i}\right)}
$$

where $f_{0}$ is the carrier frequency. It is assumed that none of the even order terms in the passband volterra series contribute to the output signal, as they produce frequency components which can be removed by a filter centered at $f_{0}$. So, the equivalent baseband representation in (2.6) contains only the odd order terms. The discrete time representation of the volterra series is

$$
y(n)=\sum_{p=0}^{P-1} \sum_{l_{1}} \sum_{l_{2}} \ldots \sum_{l_{2 p+1}} h_{2 p+1}\left(l_{1}, l_{2}, \ldots, l_{2 p+1}\right) \prod_{i=1}^{p+1} x\left(n-l_{i}\right) \prod_{i=p+2}^{2 p+1} x^{*}\left(n-l_{i}\right)
$$

As the memory length and the nonlinear order are increased, the number of kernel coefficients of the Volterra series increases exponentially. This drawback makes the Volterra series unattractive for real-time applications. Several simplified special cases of the Volterra series which are suitable for different applications exist in literature. In this section we consider three such special cases, the Wiener model, the Hammerstein model, and the memory polynomial model. The Wiener model [29] as shown in Fig. 2.3a, is a linear time-invariant (LTI) system followed by a memoryless nonlinearity (NL). The input and output signals are related as

$$
\begin{aligned}
& u(n)=\sum_{l=0}^{L-1} a_{l} x(n-l) \\
& y(n)=\sum_{\substack{k=1 \\
k \text { odd }}}^{K} b_{k}|u(n)|^{k-1} u(n)
\end{aligned}
$$




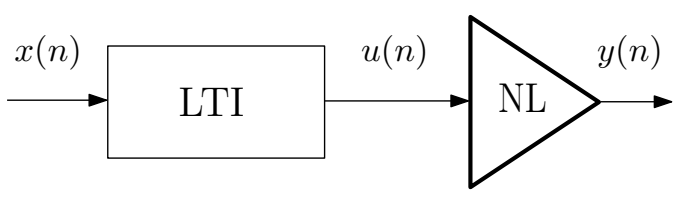

(a) The Wiener model

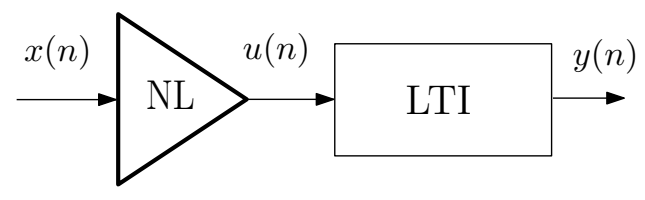

(b) The Hammerstein model

Fig. 2.3: Wiener and Hammerstein models

The Hammerstein model [30,31] as shown in Fig. 2.3b, is a memoryless nonlinearity followed by a LTI system. The input and output signals are related as

$$
\begin{aligned}
& u(n)=\sum_{\substack{k=1 \\
k \text { odd }}}^{K} b_{k}|x(n)|^{k-1} x(n) \\
& y(n)=\sum_{l=0}^{L-1} a_{l} u(n-l)
\end{aligned}
$$

The memory polynomial model $[28,32,33]$ only uses the so-called diagonal kernels of the Volterra series. In (2.8), if we assign

$$
h_{2 p+1}\left(l_{1}, l_{2}, \ldots, l_{2 p+1}\right)= \begin{cases}h_{k, q}, & \text { if } l_{1}=l_{2}=\ldots=l_{2 p+1}=q \\ 0, & \text { else }\end{cases}
$$

and $k=2 p+1$ and $K=2 P-1$, the discrete time volterra series reduces to the memory polynomial model

$$
y(n)=\sum_{q=0}^{Q-1} \sum_{\substack{k=1 \\ k \text { odd }}}^{K} h_{k, q}|x(n-q)|^{k-1} x(n-q)
$$

In [34], it is shown that memory polynomial model is equivalent to the parallel Hammerstein model and a special case of parallel Wiener model ${ }^{1}$. As the Wiener and Hammerstein models are only partially linear in parameters, the parameter estimation using the conventional least-squares approach is not applicable in a straightforward manner [30,35-38]. The memory polynomial model is linear in its parameters, and the conventional least-squares approach can be easily applied. Moreover, the memory polynomial model gives a good compromise between complexity and accuracy [39].

\footnotetext{
${ }^{1} \mathrm{~A}$ parallel Wiener/Hemmerstein model contains multiple Wiener/Hemmerstein models connected in parallel, so that the output signal is the superposition of the component outputs.
} 


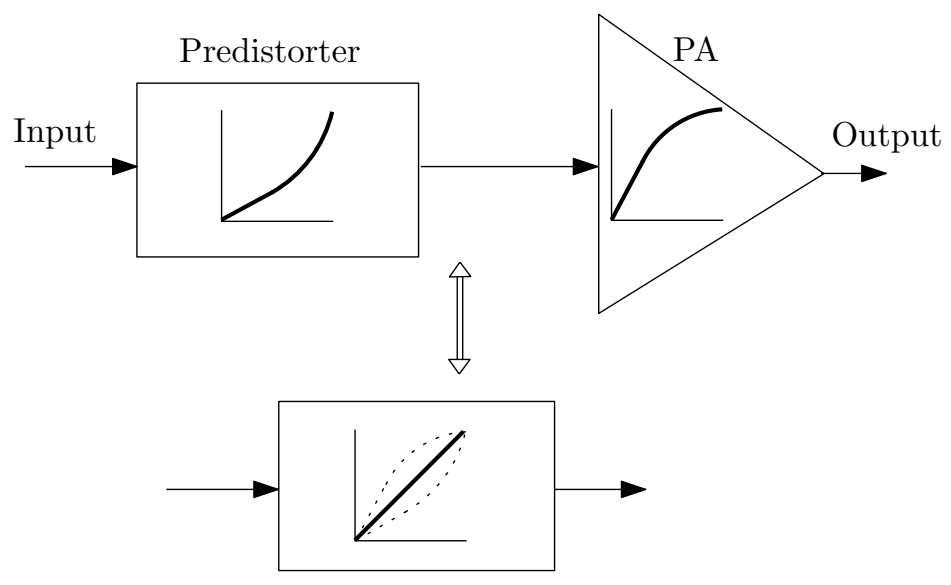

Fig. 2.4: Predistortion concept

\subsection{Digital predistortion}

The three principal types of linearization are feedforward, feedback, and predistortion $[16,17,38,40]$. The feedforward technique consists of two amplifiers (the main and error amplifiers), directional couplers, delay lines, and loop control. The input signal is first split into two paths, with one path going to the high-power main amplifier, while the other signal path goes to a delay element. The output signal from the main amplifier contains both the desired signal and distortion. The delayed portion of the input signal which is regarded as distortion free is subtracted from a scaled version of the output signal of the main amplifier. The resulting error signal ideally contains only the distortion components in the output of the main amplifier. The error signal is then amplified by the low-power high-linearity error amplifier, and then subtracted from a delayed version of the main amplifier output. This second combination ideally cancels the distortion components in the main-amplifier output while leaving the desired signal unaltered. In feedback linearization, a portion of the RF output signal from the amplifier is fed back to and subtracted from the input signal. RF feedback, envelope feedback, polar loop and cartesian feedback are some of the widely used feedback linearization techniques.

The basic idea of predistortion is to insert a nonlinear element that inversely models the amplifier's gain and phase characteristics, prior to the RF PA as shown in Fig. 2.4. The predistorter, when combined with the amplifier, produces an overall system that is linear. Predistortion can be accomplished at either RF or baseband. An RF predistorter typically creates the expansive characteristic by subtracting a compressive transfer function from a linear transfer function. Digital baseband predistortion (or simply digital predistortion) is one of the most cost effective ways among all linearization techniques, and it can exploit the considerable processing 


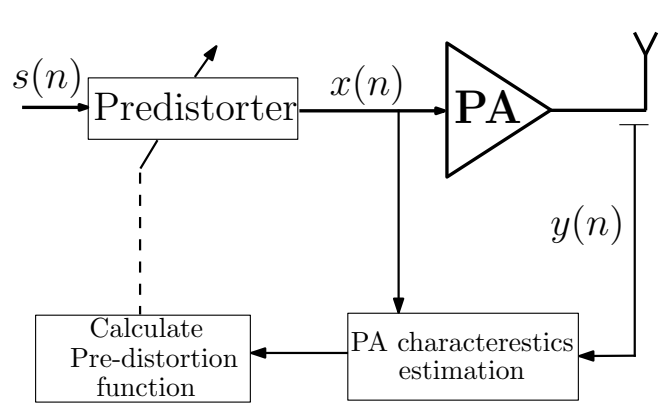

(a) Direct learning architecture

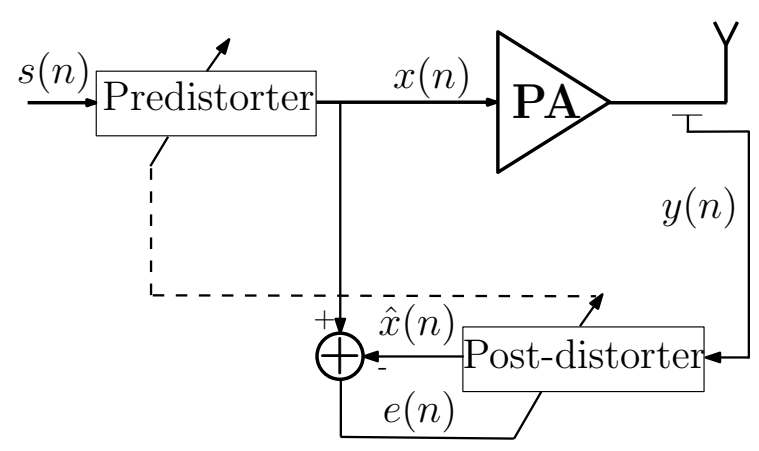

(b) Indirect learning architecture

Fig. 2.5: Direct and indirect learning architectures

power now available from DSP devices.

There are mainly two categories of digital predistortion techniques, data predistorters and signal predistorters [34]. Data predistortion is applied directly to each of the input signal constellation points. The main drawback of data predistorters is their dependence on the signal constellation and the pulse shaping filter. Moreover, data predistorters do not work well if the input signal has almost continuous amplitude levels, e.g. OFDM, WCDMA etc. In contrast to data predistorters, signal predistorters which are applied at the last stage of the baseband processing, can deal with arbitrary input waveforms. Signal predistorters can be classified in to look-up table (LUT)-based and model-based predistorters. In this work, we focus on memory polynomial model-based signal predistorters.

\subsection{Direct and indirect learning architectures}

Two methods for constructing a predistorter are commonly used, namely the direct and the indirect learning architectures as shown in Fig. $2.5^{2}$. In direct learning, the input-output relation of the power amplifier is estimated, and the predistortion is obtained directly by 'pre-inverting' the PA characteristics [32,41]. For a memory polynomial PA model, the inverse model can be expressed as [32]

$$
x(n)=\frac{1}{\beta_{0}(|x(n)|)}\left(s(n)-\sum_{q=0}^{Q-1} \beta_{q}(|x(n-q)|) x(n-q)\right),
$$

\footnotetext{
${ }^{2}$ For simplicity, the baseband equivalent models are depicted here, and they do not illustrate the digital to analog conversion and up-conversion of $x(n)$ to form the RF signal at the PA input, and the attenuation, down-conversion and analog to digital conversion of the RF signal at the PA output, to form $y(n)$.
} 
where $\beta_{q}(|x|)=\sum_{\substack{k=1 \\ k \text { odd }}}^{K} h_{k, q}|x|^{k-1}$. However, obtaining the inverse of a nonlinear system with memory is generally a difficult task. Another type of approach is to use the indirect learning architecture to design the predistorter directly. For a nonlinear system that can be expressed using Volterra series, it is shown in [42] that the $p^{\text {th }}$ order pre-inverse is identical to the $p^{\text {th }}$ order post-inverse. So, the post-inverse is used as the pre-inverse in the indirect learning architecture $[41,43]$. 


\section{Chapter 3.}

\section{Digital predistortion for MIMO transmitters}

One of the challenges in MIMO transceiver design is the cross-talk between multiple transmit/receive paths which are integrated on the same chip-set [4-7]. Electromagnetic coupling and the leakage of the modulated signal through a common local-oscillator (LO) are the main causes of the cross-talk. Several isolation techniques $[6,44-47]$ are proposed to suppress the coupling and crosstalk effects in the IC design. The usability and overall performance of those isolation techniques are dependent on the design process and the accessibility of the methods. In general, the proposed techniques may minimize the crosstalk, but they cannot completely eliminate it. In multi-antenna transmitters, the effect of this cross-talk which takes place before the power amplifier (PA) is non-linear, due to the non-linear behavior of the PA [8]. In the presence of non-linear cross-talk in MIMO transmitters, the performance of the conventional DPD (C-DPD) algorithms degrades considerably.

A cross-over predistortion (CO-DPD) algorithm [8] that compensates the cross-talk and non-linearity of the PAs significantly improves the quality of predistortion compared to the C-DPD. But, as the cross-over model for MIMO transmitters ignores the higher order cross-terms between the input signals, the linearization performance of CO-DPD degrades, especially at high cross-talk levels. We show that the cross-over coefficients extracted using the indirect learning architecture form an accurate post-distorter for a MIMO transmitter, but not an accurate predistorter. We proposed a cross-talk canceling predistorter (CTC-DPD) which accurately models and compensates cross-talk and non-linearity in [9]. A comprehensive study of the linearization performance and computational complexity of MIMO-DPDs (ie., CO-DPD and CTC-DPD) and C-DPD was presented in [10]. An architecture to compensate post-amplifier and pre-amplifier crosstalk by matrix operations before and after the predistorters was proposed in [48]. But, no further details of its parameter identification and operation was presented in this patent. In [11], a so-called streamlined cross-over DPD is proposed, which is similar to CTC-DPD, but no detailed analysis is given. Generalized memory polynomial models for linear and nonlinear crosstalk in MIMO transmitters are discussed in [49].

The linearization performances of CO-DPD and CTC-DPD for a two-antenna trans- 


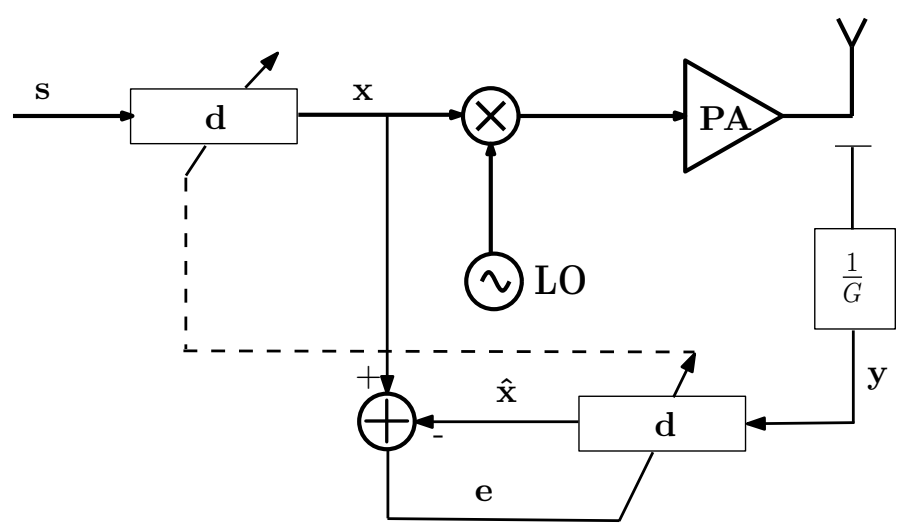

Fig. 3.1: Indirect learning architecture for predistortion

mitter are evaluated through measurements with QPSK, 16-QAM, UMTS and LTE signals. Performance measures such as in-band normalized mean squared error (NMSE $\left.E_{\mathrm{IB}}\right)$, error vector magnitude (EVM), adjacent channel leakage ratio (ACLR) and adjacent channel error power ratio (ACEPR) are analyzed in section 3.5. It is found that CTC-DPD achieves a slight performance improvement over CO-DPD at higher cross-talk levels, when the PA is operated with high non-linearity with signals having low peak-to-average-power ratios (PAPR). In the presence of frequency selective cross-talk, an extension of CTC-DPD which can pre-cancel such cross-talk, performs considerably better than CO-DPD, which can not take the the frequency selectivity of the cross-talk in to account.

The architectures and the parameter identification methods of CO-DPD and CTCDPD can be extended to any number of transmit paths. The CO-DPD requires $N_{t}^{2}$ branch predistorters ( $N_{t}$ is the number of transmit paths), whereas CTC-DPD requires $N_{t}$ predistorters and a cross-talk pre-cancellation block which involves the inversion of an $N_{t} \times N_{t}$ matrix. Because of this, CTC-DPD is computationally less complex compared to CO-DPD. A detailed discussion on the complexity of these schemes for a two-antenna transmitter is presented in chapter 5 .

\subsection{Power amplifier non-linearity and RF cross-talk in MIMO transmitters}

The indirect learning architecture of predistortion is shown in Fig. $3.1^{1}$. The process of predistortion can be described as

$$
y=h(x), x=d(s) \text { and } h(d(s))=s
$$

\footnotetext{
${ }^{1}$ For simplicity, this diagram does not illustrate down-conversion of the output RF signal of the PA to form the baseband equivalent output signal.
} 


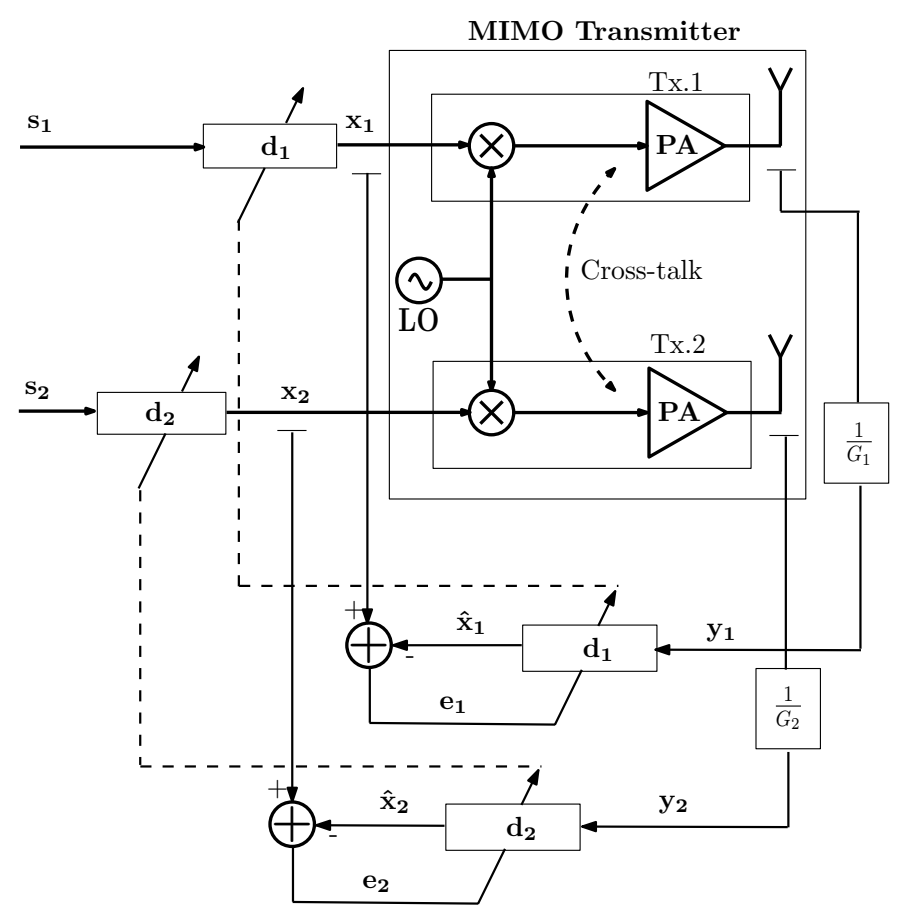

Fig. 3.2: Conventional predistortion (C-DPD) in the presence of non-linear crosstalk

where $s$ and $x$ are the input and predistorted baseband signals, $y$ is the complex envelope of the RF signal at the PA output attenuated by $G$ which is the small signal gain of the PA, $h($.$) is the non-linear response of the PA and d($.$) is the predistortion$ function. As the multiple RF paths in a MIMO transmitter operate at the same frequency, a limited RF isolation between the paths in MIMO transmitters leads to an unavoidable cross-talk between the RF signals. The PA non-linearity and the RF cross-talk between the different paths of the transmitters are the two major sources of impairments at the transmit side of a MIMO communication system [4]. This cross-talk, which occurs before the PA in the transmit path, has a non-linear effect at the output of the PA and it is thus termed as non-linear cross-talk. On the other hand, the antenna crosstalk, which is the effect of the transmitted signal from one antenna element to another antenna element, is modelled as linear cross-talk. The compensation of linear cross-talk is done mostly at the receiver side concurrently for the composite linear crosstalk generated at the transmitter and receiver antennas and by the channel [8]. In our work, we do not consider the linear cross-talk, as it has no effect on predistortion. The PA outputs including only the effects of PA nonlinearity and non-linear cross-talk can be expressed as

$$
\begin{aligned}
& y_{1}=h_{1}\left(x_{1}+\alpha_{1} x_{2}\right) \\
& y_{2}=h_{2}\left(x_{2}+\alpha_{2} x_{1}\right)
\end{aligned}
$$


where, $\alpha_{1}$ and $\alpha_{2}$ are the coupling factors which model the leakage of the RF signal from one transmit path to the other. $h_{1}($.$) and h_{2}($.$) are the nonlinear PA responses.$ In the case of a C-DPD, the predistorter functions are obtained independently for each transmitter, as shown in Fig. 3.2. $d_{1}($.$) and d_{2}($.$) are the inverses of h_{1}($.$) and$ $h_{2}($.$) respectively. The outputs of the PAs are$

$$
\begin{gathered}
y_{1}=h_{1}\left(d_{1}\left(s_{1}\right)+\alpha_{1} x_{2}\right) \neq s_{1} \\
y_{2}=h_{2}\left(d_{2}\left(s_{2}\right)+\alpha_{2} x_{1}\right) \neq s_{2} .
\end{gathered}
$$

This shows that the non-linear cross-talk adversely affects the performance of CDPD. In the next section, we discuss the cross-over and cross-talk canceling predistortion techniques (Fig. 3.3 and Fig. 3.4) which can pre-compensate the non-linear cross-talk and the PA non-linearity.

\subsection{Cross-over and cross-talk canceling predistortion techniques}

In our investigations, we use the memory polynomial model for describing the dynamic non-linear behavior of the PA according to

$$
y(n)=\sum_{q=0}^{Q-1} \sum_{\substack{k=1 \\ k \text { odd }}}^{K} h_{k, q}|x(n-q)|^{k-1} x(n-q)+w(n)
$$

where $x(n), y(n)$ and $w(n)$ are the complex envelopes of the input and output signals of the PA and the measurement noise, respectively. $h_{k, q}$ is the $k^{\text {th }}$ polynomial coefficient of the $q^{\text {th }}$ filter tap, and $K$ and $Q$ are the maximum polynomial order and memory depth, respectively. For a block of $N$ samples, (3.4) can be expressed in a matrix form as

$$
\boldsymbol{y}=\boldsymbol{A}_{x} \boldsymbol{h}+\boldsymbol{w}
$$

where $\boldsymbol{y}=[y(1) y(2) \ldots y(N)]^{T}$ and $\boldsymbol{w}=[w(1) w(2) \ldots w(N)]^{T}$ are vectors representing the complex envelope signals of the PA output signal and the measurement noise, respectively. $\boldsymbol{h}=\left[\begin{array}{llllll}h_{1,0} & h_{3,0} & \ldots & h_{K, 0} & \ldots & h_{1, Q} \\ h_{3, Q} & \ldots & h_{K, Q}\end{array}\right]^{T}$ is the $L \times 1$ vector of the memory polynomial coefficients which model the PA response, where $L=Q(K+1) / 2$. The data matrix $\boldsymbol{A}_{\boldsymbol{x}}$ is

$$
\boldsymbol{A}_{\boldsymbol{x}}=\left[\begin{array}{llllll}
\boldsymbol{a}_{x}{ }^{0} & \boldsymbol{a}_{x}{ }^{1} & \ldots & \boldsymbol{a}_{x}{ }^{q} & \ldots & \boldsymbol{a}_{x}{ }^{Q-1}
\end{array}\right]
$$

where

$$
\boldsymbol{a}_{x}{ }^{q}=\left[\begin{array}{cccc}
\mathbf{0}_{q \times 1} & \mathbf{0}_{q \times 1} & \ldots & \mathbf{0}_{q \times 1} \\
a_{1}(x(1)) & a_{3}(x(1)) & \ldots & a_{K}(x(1)) \\
\ldots & \ldots & \ldots & \ldots \\
a_{1}(x(N-q)) & a_{3}(x(N-q)) & \ldots & a_{K}(x(N-q))
\end{array}\right]
$$




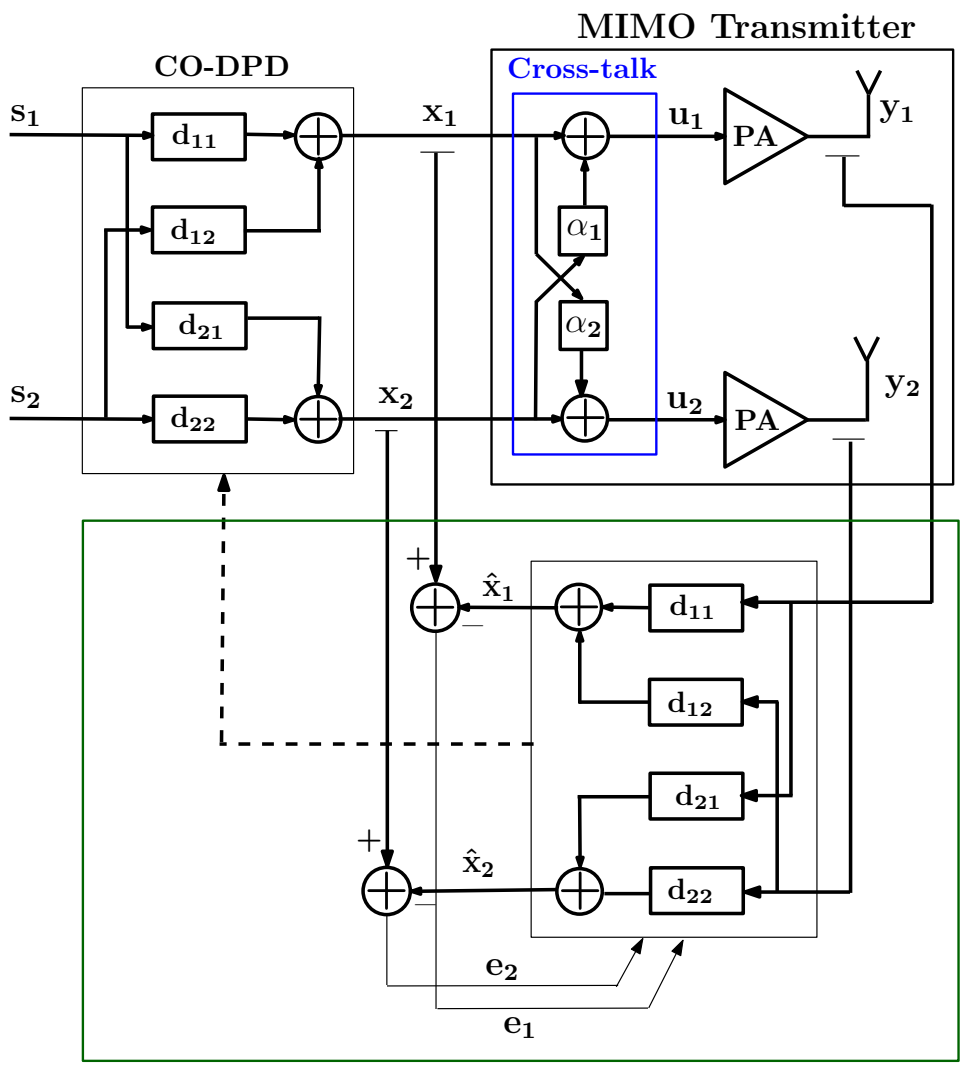

DPD Identification

Fig. 3.3: Cross-over digital predistortion (CO-DPD) for 2 antenna transmitter

and $a_{k}(x)=|x|^{k-1} \cdot x$. The output signal vector of the post-distorter $\boldsymbol{d}$ can be written as

$$
\hat{\boldsymbol{x}}=\boldsymbol{A}_{y} \boldsymbol{d}
$$

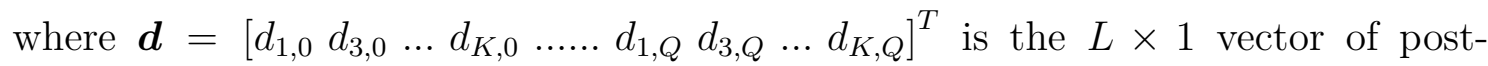
distorter coefficients. The least-squares (LS) estimate of the post-distorter coefficients, which minimizes the energy in the error signal $\boldsymbol{e}$ is

$$
\boldsymbol{d}=\operatorname{pinv}\left(\boldsymbol{A}_{y}\right) \boldsymbol{x}
$$

where $\boldsymbol{x}=[x(1) x(2) \ldots x(N)]^{T}$ is the input signal vector and the pseudo-inverse of $\boldsymbol{A}_{\boldsymbol{y}}$ is

$$
\operatorname{pinv}\left(\boldsymbol{A}_{\boldsymbol{y}}\right)=\left(\boldsymbol{A}_{\boldsymbol{y}}{ }^{H} \boldsymbol{A}_{\boldsymbol{y}}\right)^{-1} \boldsymbol{A}_{\boldsymbol{y}}{ }^{H}
$$

This method is called data least squares and is explained in more detail in [50]. In the indirect learning architecture which we use in this work, the post-distorter is used as the predistorter, as discussed earlier in chapter 2 .

The cross-over model, which was proposed in [8], is an extension of the memory polynomial model for a MIMO transmitter which deals with the cross-talk and non- 
linearity. For a two-antenna transmitter it can be expressed as

$$
\left[\begin{array}{ll}
\boldsymbol{y}_{1} & \boldsymbol{y}_{2}
\end{array}\right]=\left[\begin{array}{ll}
\boldsymbol{A}_{x_{1}} & \boldsymbol{A}_{x_{2}}
\end{array}\right]\left[\begin{array}{ll}
\boldsymbol{h}_{1,1} & \boldsymbol{h}_{2,1} \\
\boldsymbol{h}_{1,2} & \boldsymbol{h}_{2,2}
\end{array}\right]+\left[\begin{array}{ll}
\boldsymbol{w}_{1} & \boldsymbol{w}_{2}
\end{array}\right],
$$

where $\boldsymbol{y}_{1}, \boldsymbol{y}_{2}$ and $\boldsymbol{w}_{1}, \boldsymbol{w}_{2}$ are the $N \times 1$ vectors of PA output signal and the measurement noise, respectively. $\boldsymbol{A}_{y_{1}}$ and $\boldsymbol{A}_{y_{2}}$ are data matrices of size $N \times L$, $\boldsymbol{h}_{i, j}, i, j \in\{1,2\}$ are the cross-over polynomial coefficient vectors of size $L \times 1$. This model does not take into account all the high-order cross terms between the input signals in the polynomials, as it models the MIMO system as the sum of weighted nonlinear functions. Hence, it is anticipated that this model would not offer good accuracy in modeling a multiple antenna transmitter, especially for strong cross-talk effects between the branches of the MIMO system [51]. The cross-over predistorter (CO-DPD) is based on the cross-over model and it is shown in Fig. 3.3 applying the indirect learning architecture. The CO-DPD coefficients are computed as

$$
\left[\begin{array}{ll}
\boldsymbol{d}_{1,1} & \boldsymbol{d}_{2,1} \\
\boldsymbol{d}_{1,2} & \boldsymbol{d}_{2,2}
\end{array}\right]=\operatorname{pinv}\left(\left[\begin{array}{ll}
\boldsymbol{A}_{y_{1}} & \boldsymbol{A}_{y_{2}}
\end{array}\right]\right)\left[\begin{array}{ll}
\boldsymbol{x}_{1} & \boldsymbol{x}_{2}
\end{array}\right]
$$

where $\boldsymbol{x}_{1}$ and $\boldsymbol{x}_{2}$ are the input signal vectors and $\boldsymbol{d}_{1,1}, \boldsymbol{d}_{1,2}, \boldsymbol{d}_{2,1}$ and $\boldsymbol{d}_{2,2}$ are the CO-DPD coefficient vectors. The error in the estimation of DPD coefficients and the linearization performance measures, which will be defined in section 3.4 and section 3.5, suggest that the cross-over coefficients extracted according to (3.11) form an accurate post-distorter for a MIMO transmitter, but not an accurate predistorter. This will be discussed again in section 3.5.

A cross-talk canceling predistorter (CTC-DPD), which accurately pre-compensates cross-talk and non-linearity of the MIMO transmitter was proposed in [9]. As the complex envelopes of the input signals to the PAs are

$$
\begin{aligned}
& u_{1}(n)=x_{1}(n)+\alpha_{1} x_{2}(n) \\
& u_{2}(n)=x_{2}(n)+\alpha_{2} x_{1}(n)
\end{aligned}
$$

the cross-talk can be pre-cancelled as

$$
\begin{aligned}
& x_{1}(n)=\frac{1}{1-\hat{\alpha}_{1} \hat{\alpha}_{2}}\left(v_{1}(n)-\hat{\alpha}_{1} v_{2}(n)\right) \\
& x_{2}(n)=\frac{1}{1-\hat{\alpha}_{1} \hat{\alpha}_{2}}\left(v_{2}(n)-\hat{\alpha}_{2} v_{1}(n)\right)
\end{aligned}
$$

where $\hat{\alpha}_{1}$ and $\hat{\alpha}_{2}$ are the estimates of the coupling factors. This condition makes $u_{1}(n)$ and $u_{2}(n)$ equal to $v_{1}(n)$ and $v_{2}(n)$ respectively, if the estimation of the coupling factors is accurate. The estimation of the coupling factors is explained in the following part of this section, and the estimation accuracy is discussed in Appendix B. The indirect learning architecture for CTC-DPD is illustrated in Fig. 3.4. The DPD coefficients for CTC-DPD can be estimated using the least squares method as 


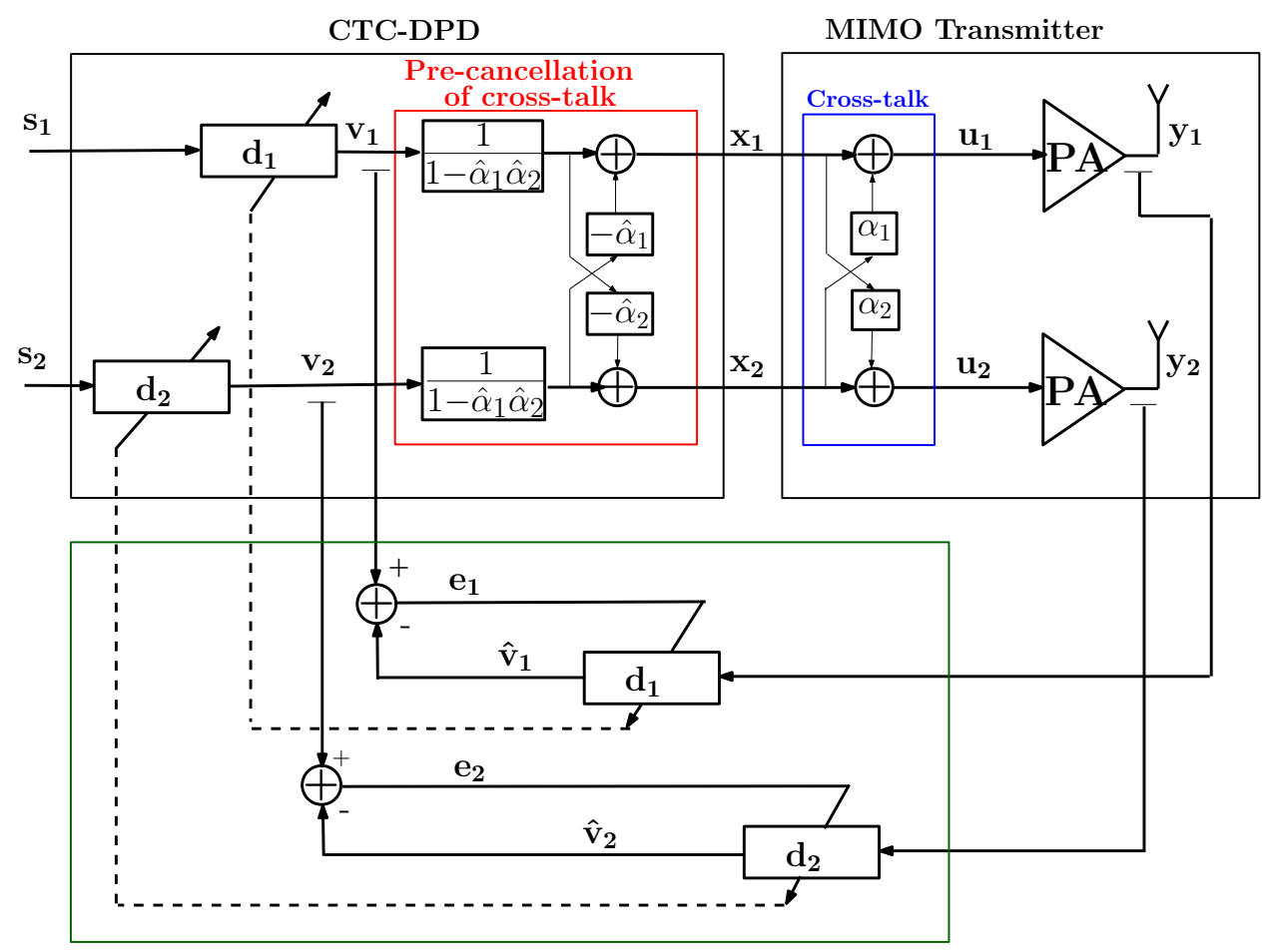

DPD Identification

Fig. 3.4: Cross-talk canceling digital predistortion (CTC-DPD) for 2 antenna transmitter

$$
\begin{aligned}
& \boldsymbol{d}_{1}=\operatorname{pinv}\left(\boldsymbol{A}_{y_{1}}\right) \boldsymbol{v}_{1} \\
& \boldsymbol{d}_{2}=\operatorname{pinv}\left(\boldsymbol{A}_{y_{2}}\right) \boldsymbol{v}_{2}
\end{aligned}
$$

where $\boldsymbol{v}_{1}$ and $\boldsymbol{v}_{2}$ are the vectors of length $N$, containing the samples of $v_{1}(n)$ and $v_{2}(n)$ respectively.

The CO-DPD and CTC-DPD structures can be easily extended to any number of transmit antennas. As an example, we briefly discuss CO-DPD and CTC-DPD structures for a 4 antenna transmitter, here. However we consider only the two antenna transmitter case in the rest of the thesis, for simplicity. The cross-over model for a 4 antenna transmitter would be a simple extension of (3.10), with 16 branch polynomials

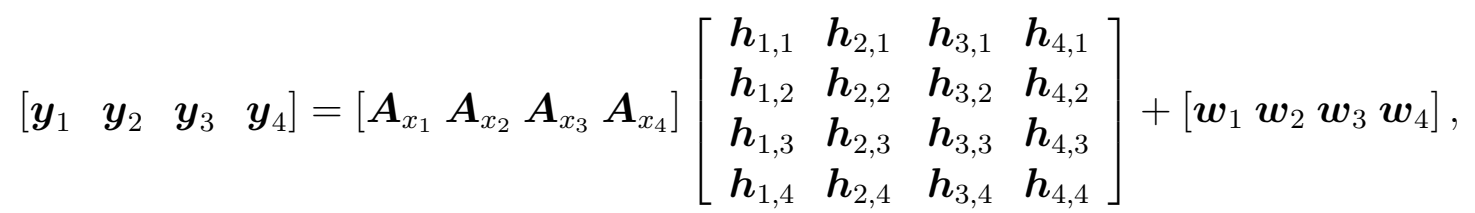

where $\boldsymbol{h}_{i, j}, i, j \in\{1,2,3,4\}$ are the $L \times 1$ vectors containing branch polynomial coefficients. $\boldsymbol{y}_{i}$ and $\boldsymbol{w}_{i}$ are the output signal and noise vectors of size $N \times 1$ and 


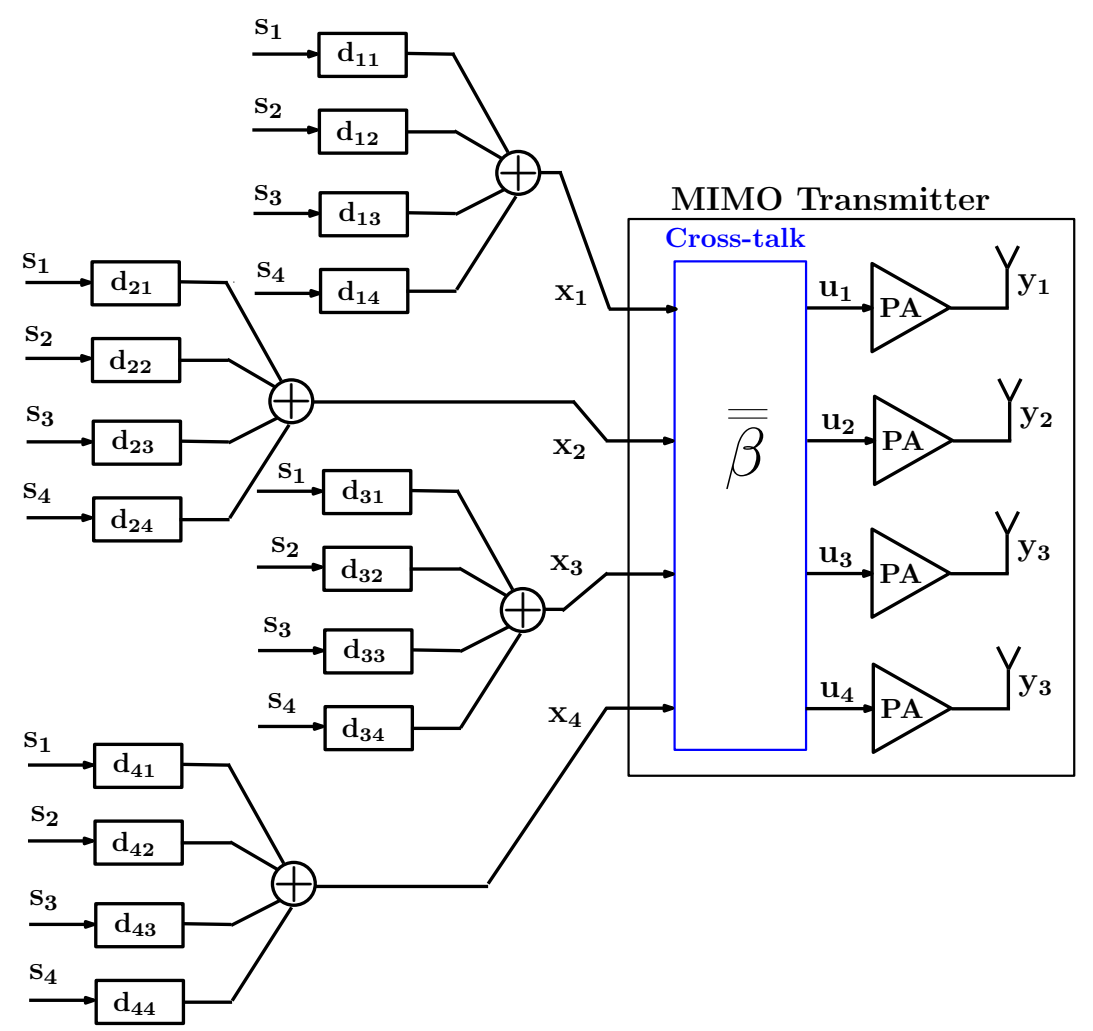

Fig. 3.5: CO-DPD for 4 antenna Transmitter

$\boldsymbol{A}_{x_{i}}$ are the data matrices of size $N \times L$. The CO-DPD structures for a 4 antenna transmitter with 16 branch predistorters is shown in Fig. 3.5. To construct the CTCDPD for a 4 antenna transmitter, we consider the complex envelopes of the input signals to the PAs which can be expressed as

$$
\boldsymbol{u}_{4 T X}(n)=\overline{\overline{\boldsymbol{\beta}}} \boldsymbol{x}_{4 T X}(n)
$$

where $\boldsymbol{u}_{4 T X}(n)=\left[u_{1}(n) u_{2}(n) u_{3}(n) u_{4}(n)\right]^{T}, \overline{\overline{\boldsymbol{\beta}}}=\left[\begin{array}{cccc}1 & \beta_{12} & \beta_{13} & \beta_{14} \\ \beta_{21} & 1 & \beta_{23} & \beta_{24} \\ \beta_{31} & \beta_{32} & 1 & \beta_{34} \\ \beta_{41} & \beta_{42} & \beta_{43} & 1\end{array}\right]$ and $\boldsymbol{x}_{4 T X}(n)=\left[x_{1}(n) x_{2}(n) x_{3}(n) x_{4}(n)\right]^{T} \cdot \beta_{i j}$ is the coupling factor ${ }^{2}$ which model the cross-talk from the $j^{\text {th }}$ transmit path to the $i^{\text {th }}$ transmit path. The CTCDPD structure for a 4 antenna transmitter is shown in Fig. 3.6. The cross-talk precancellation can be done using the inverse of an estimate of the cross-talk matrix, $\hat{\overline{\overline{\boldsymbol{\beta}}}}$. The DPD coefficients can be separately estimated similar to (3.14).

\footnotetext{
${ }^{2}$ The coupling factors $\beta_{i j}$ s here are similar to $\alpha_{i} \mathrm{~s}$ in $(3.12)$
} 


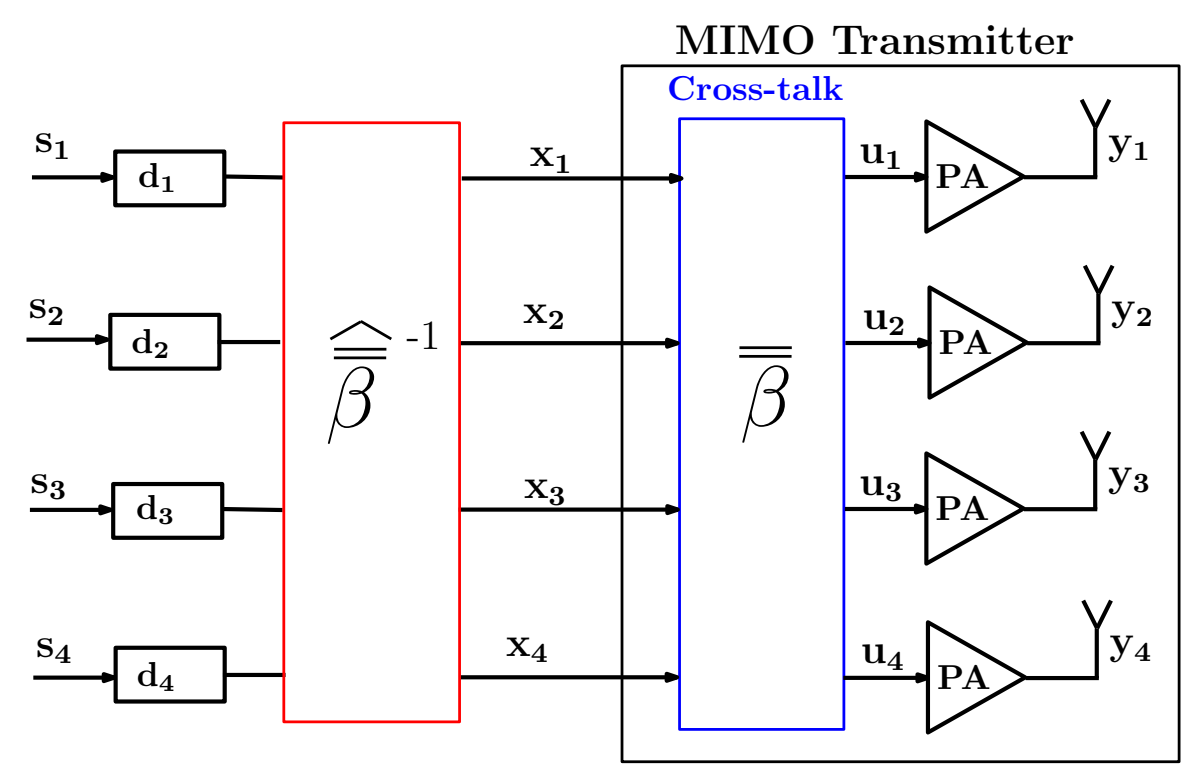

Fig. 3.6: CTC-DPD for 4 antenna Transmitter

\subsubsection{Estimation of coupling factors for CTC-DPD}

The estimates of coupling factors, $\hat{\alpha}_{1}$ and $\hat{\alpha}_{2}$ can be obtained from the linear section of the PA response. We define a vector operator $\mathcal{F}_{T, y}$ as follows: $\mathcal{F}_{T, y}(\boldsymbol{x})$ is a vector containing only those elements of $\boldsymbol{x}$ corresponding to index $n$ which satisfy $|y(n)|<T$. This is equivalent to the removal of the elements from $\boldsymbol{x}$ for which the magnitude of the corresponding elements of $\boldsymbol{y}$ are larger than a threshold $T$. Now we define

$$
\begin{array}{ll}
\tilde{\boldsymbol{y}}_{1}=\mathcal{F}_{T, y_{1}}\left(\boldsymbol{y}_{1}\right), & \grave{\boldsymbol{y}}_{2}=\mathcal{F}_{T, y_{2}}\left(\boldsymbol{y}_{2}\right) \\
\tilde{\boldsymbol{x}}_{1}=\mathcal{F}_{T, y_{1}}\left(\boldsymbol{x}_{1}\right), & \grave{\boldsymbol{x}}_{1}=\mathcal{F}_{T, y_{2}}\left(\boldsymbol{x}_{1}\right) \\
\tilde{\boldsymbol{x}}_{2}=\mathcal{F}_{T, y_{1}}\left(\boldsymbol{x}_{2}\right), & \grave{\boldsymbol{x}}_{2}=\mathcal{F}_{T, y_{2}}\left(\boldsymbol{x}_{2}\right) .
\end{array}
$$

$\tilde{\boldsymbol{y}}_{1}$ is the PA output signal vector after the removal of the elements with magnitude larger than $T . \quad \tilde{\boldsymbol{x}}_{1}$ and $\tilde{\boldsymbol{x}}_{2}$ are the input signal vectors after the removal of the elements corresponding to output signal values with a magnitude larger than $T$. The threshold $T$ is chosen such that $\tilde{\boldsymbol{x}}_{1}, \tilde{\boldsymbol{x}}_{2}$ and $\tilde{\boldsymbol{y}}_{1}$ contain the input and output signal values for which the PA in the first transmit path is operating in the linear region. Similarly, $\grave{\boldsymbol{x}}_{1}, \grave{\boldsymbol{x}}_{2}$ and $\grave{\boldsymbol{y}}_{2}$ contain the input and output signal values for which the PA in the second transmit path is operating in the linear region. So the newly constructed signal vectors are related as

$$
\begin{aligned}
& \tilde{\boldsymbol{y}}_{1}=c_{1}\left(\tilde{\boldsymbol{x}}_{1}+\alpha_{1} \tilde{\boldsymbol{x}}_{2}\right)+\tilde{\boldsymbol{n}}_{1} \\
& \grave{\boldsymbol{y}}_{2}=c_{2}\left(\grave{\boldsymbol{x}}_{2}+\alpha_{2} \grave{\boldsymbol{x}}_{1}\right)+\grave{\boldsymbol{n}}_{2}
\end{aligned}
$$


where $c_{1}$ and $c_{2}$ are the small signal gains of the PAs in the linear region and $\tilde{\boldsymbol{n}}_{\mathbf{1}}$ and $\grave{\boldsymbol{n}}_{\mathbf{2}}$ represent the measurement noise. Note that $c_{1}$ and $c_{2}$ are not necessarily equal to unity, as they depend on the choice of the attenuation factors $G_{1}$ and $G_{2}$. (3.18) can be written as

$$
\begin{aligned}
& \tilde{\boldsymbol{y}}_{1}=\left[\begin{array}{ll}
\tilde{\boldsymbol{x}}_{1} & \tilde{\boldsymbol{x}}_{2}
\end{array}\right]\left[\begin{array}{c}
c_{1} \\
c_{1} \alpha_{1}
\end{array}\right]+\tilde{\boldsymbol{n}}_{1} \\
& \grave{\boldsymbol{y}}_{2}=\left[\begin{array}{ll}
\grave{\boldsymbol{x}}_{2} & \grave{\boldsymbol{x}}_{1}
\end{array}\right]\left[\begin{array}{c}
c_{2} \\
c_{2} \alpha_{2}
\end{array}\right]+\grave{\boldsymbol{n}}_{2} .
\end{aligned}
$$

The least square estimates of $\left[\begin{array}{c}c_{1} \\ c_{1} \alpha_{1}\end{array}\right]$ and $\left[\begin{array}{c}c_{2} \\ c_{2} \alpha_{2}\end{array}\right]$ can be obtained as

$$
\begin{aligned}
\tilde{\boldsymbol{\alpha}} & =\operatorname{pinv}\left(\left[\begin{array}{ll}
\tilde{\boldsymbol{x}}_{1} & \tilde{\boldsymbol{x}}_{2}
\end{array}\right]\right) \tilde{\boldsymbol{y}}_{1} \\
\grave{\boldsymbol{\alpha}} & =\operatorname{pinv}\left(\left[\begin{array}{ll}
\grave{\boldsymbol{x}}_{2} & \grave{\boldsymbol{x}}_{1}
\end{array}\right]\right) \grave{\boldsymbol{y}}_{2} .
\end{aligned}
$$

The estimates of coupling factors $\hat{\alpha}_{1}$ and $\hat{\alpha}_{2}$ are

$$
\hat{\alpha}_{1}=\frac{\tilde{\boldsymbol{\alpha}}(2)}{\tilde{\boldsymbol{\alpha}}(1)}, \quad \hat{\alpha}_{2}=\frac{\grave{\boldsymbol{\alpha}}(2)}{\grave{\boldsymbol{\alpha}}(1)} .
$$

\subsubsection{Frequency selective cross-talk}

The electromagnetic and capacitive coupling between the transmitters are, in general frequency dependent processes. As signals with higher frequencies usually experience higher losses in the coupling path, a low-pass filter can be used to model this frequency dependency. We model the frequency selective coupling using a finite impulse response (FIR) filter.

$$
\begin{aligned}
& u_{1}(n)=x_{1}(n)+\sum_{j=0}^{M-1} \alpha_{1, j} x_{2}(n-j) \\
& u_{2}(n)=x_{2}(n)+\sum_{j=0}^{M-1} \alpha_{2, j} x_{1}(n-j) .
\end{aligned}
$$

(3.22) can be written as

$$
\begin{aligned}
& \boldsymbol{u}_{1}=\boldsymbol{x}_{1}+\boldsymbol{B}_{x_{2}} \boldsymbol{\alpha}_{1} \\
& \boldsymbol{u}_{2}=\boldsymbol{x}_{2}+\boldsymbol{B}_{x_{1}} \boldsymbol{\alpha}_{2} .
\end{aligned}
$$

where $\boldsymbol{u}_{1}$ and $\boldsymbol{u}_{2}$ are vectors of complex envelopes of the input signals to the PAs, $\boldsymbol{\alpha}_{1}=\left[\begin{array}{llll}\alpha_{1,0} & \alpha_{1,1} & \ldots & \alpha_{1, M-1}\end{array}\right]^{T}$ and $\boldsymbol{\alpha}_{2}=\left[\begin{array}{llll}\alpha_{2,0} & \alpha_{2,1} & \ldots & \alpha_{2, M-1}\end{array}\right]^{T}$ are the vectors of FIR filter coefficients modeling the frequency selective cross-talk, and

$$
\boldsymbol{B}_{x_{i}}=\left[\begin{array}{ccclc}
x_{i}(1) & 0 & 0 & \ldots & 0 \\
x_{i}(2) & x_{i}(1) & 0 & \ldots & 0 \\
. \ddot{n} & . . & . . & \ldots & . . \\
x_{i}(N) & x_{i}(N-1) & x_{i}(N-2) & \ldots & x_{i}(N-M+1)
\end{array}\right]
$$


for $i=1,2$. We follow the same method introduced in section 3.2.1 to estimate the frequency selective coupling filter coefficients. The sections of input and output signal vectors and $\boldsymbol{B}_{x_{i}}$ matrices, corresponding to the linear regions of the PAs, are selected as

$$
\begin{array}{cl}
\tilde{\boldsymbol{y}}_{1}=\mathcal{F}_{T, y_{1}}\left(\boldsymbol{y}_{1}\right), & \tilde{\boldsymbol{y}}_{2}=\mathcal{F}_{T, y_{2}}\left(\boldsymbol{y}_{2}\right) \\
\tilde{\boldsymbol{x}}_{1}=\mathcal{F}_{T, y_{1}}\left(\boldsymbol{x}_{1}\right), & \tilde{\boldsymbol{x}}_{2}=\mathcal{F}_{T, y_{2}}\left(\boldsymbol{x}_{2}\right) \\
\tilde{\boldsymbol{B}}_{x_{2}}=\mathcal{F}_{T, y_{1}}\left(\boldsymbol{B}_{x_{2}}\right), & \tilde{\boldsymbol{B}}_{x_{1}}=\mathcal{F}_{T, y_{2}}\left(\boldsymbol{B}_{x_{1}}\right) .
\end{array}
$$

Note that $\mathcal{F}_{T, y}\left(\boldsymbol{B}_{x_{i}}\right)$ retains the rows of $\boldsymbol{B}_{x_{i}}$ corresponding to the linear region. The newly constructed signal vectors are related as

$$
\begin{aligned}
& \tilde{\boldsymbol{y}}_{1}=c_{1}\left(\tilde{\boldsymbol{x}}_{1}+\tilde{\boldsymbol{B}}_{x_{2}} \boldsymbol{\alpha}_{1}\right)+\tilde{\boldsymbol{n}}_{1} \\
& \tilde{\boldsymbol{y}}_{2}=c_{2}\left(\tilde{\boldsymbol{x}}_{2}+\tilde{\boldsymbol{B}}_{x_{1}} \boldsymbol{\alpha}_{2}\right)+\tilde{\boldsymbol{n}}_{2}
\end{aligned}
$$

where $c_{1}$ and $c_{2}$ are the small signal gains of the PAs in the linear region. (3.26) can be written as

$$
\begin{aligned}
& \tilde{\boldsymbol{y}}_{1}=\left[\begin{array}{ll}
\tilde{\boldsymbol{x}}_{1} & \tilde{\boldsymbol{B}}_{x_{2}}
\end{array}\right]\left[\begin{array}{c}
c_{1} \\
c_{1} \boldsymbol{\alpha}_{1}
\end{array}\right]+\tilde{\boldsymbol{n}}_{1} \\
& \tilde{\boldsymbol{y}}_{2}=\left[\begin{array}{ll}
\tilde{\boldsymbol{x}}_{2} & \tilde{\boldsymbol{B}}_{x_{1}}
\end{array}\right]\left[\begin{array}{c}
c_{2} \\
c_{2} \boldsymbol{\alpha}_{2}
\end{array}\right]+\tilde{\boldsymbol{n}}_{2} .
\end{aligned}
$$

The least square estimates of $\left[\begin{array}{c}c_{1} \\ c_{1} \boldsymbol{\alpha}_{1}\end{array}\right]$ and $\left[\begin{array}{c}c_{2} \\ c_{2} \boldsymbol{\alpha}_{2}\end{array}\right]$ can be obtained as

$$
\begin{aligned}
\tilde{\boldsymbol{\alpha}}_{\mathbf{1}} & =\operatorname{pinv}\left(\left[\begin{array}{ll}
\tilde{\boldsymbol{x}}_{1} & \tilde{\boldsymbol{B}}_{x_{2}}
\end{array}\right]\right) \tilde{\boldsymbol{y}}_{1} \\
\tilde{\boldsymbol{\alpha}}_{\mathbf{2}} & =\operatorname{pinv}\left(\left[\begin{array}{ll}
\tilde{\boldsymbol{x}}_{2} & \tilde{\boldsymbol{B}}_{x_{1}}
\end{array}\right]\right) \tilde{\boldsymbol{y}}_{2} .
\end{aligned}
$$

The estimates of coupling filter coefficients $\hat{\boldsymbol{\alpha}}_{1}$ and $\hat{\boldsymbol{\alpha}}_{2}$ can be obtained by dividing the last $M$ elements by the first element of $\tilde{\boldsymbol{\alpha}}_{\mathbf{1}}$ and $\tilde{\boldsymbol{\alpha}}_{\mathbf{2}}$ respectively, i.e.,

$$
\hat{\boldsymbol{\alpha}}_{1}=\frac{\tilde{\boldsymbol{\alpha}}_{1}(2: M+1)}{\tilde{\boldsymbol{\alpha}}_{1}(1)}, \quad \hat{\boldsymbol{\alpha}}_{2}=\frac{\tilde{\boldsymbol{\alpha}}_{2}(2: M+1)}{\tilde{\boldsymbol{\alpha}}_{2}(1)} .
$$

The frequency selective cross-talk as expressed in (3.22) can be written in the $z$ domain as

$$
\begin{aligned}
& U_{1}(z)=X_{1}(z)+\sum_{j=0}^{M-1} \alpha_{1, j} z^{-j} X_{2}(z) \\
& U_{2}(z)=X_{2}(z)+\sum_{j=0}^{M-1} \alpha_{2, j} z^{-j} X_{1}(z)
\end{aligned}
$$

The cross-talk pre-cancellation is done as

$$
\begin{aligned}
& X_{1}(z)=A_{11}(z) V_{1}(z)+A_{12}(z) V_{2}(z) \\
& X_{2}(z)=A_{22}(z) V_{2}(z)+A_{21}(z) V_{1}(z)
\end{aligned}
$$


where $U_{i}(z), X_{i}(z)$ and $V_{i}(z)$ are the $z$-transforms of $u_{i}(n), x_{i}(n)$ and $v_{i}(n)$ respectively, and

$$
\begin{aligned}
& A_{11}(z)=A_{22}(z)=\frac{1}{1-\sum_{k=0}^{2 M-2} c_{k} z^{-k}} \\
& A_{12}(z)=\frac{-\sum_{j=0}^{M-1} \alpha_{1, j} z^{-j}}{1-\sum_{k=0}^{2 M-2} c_{k} z^{-k}} \\
& A_{21}(z)=\frac{-\sum_{j=0}^{M-1} \alpha_{2, j} z^{-j}}{1-\sum_{k=0}^{2 M-2} c_{k} z^{-k}}
\end{aligned}
$$

where

$$
\sum_{k=0}^{2 M-2} c_{k} z^{-k}=\sum_{i=0}^{M-1} \sum_{j=0}^{M-1} \hat{\alpha}_{1, i} \hat{\alpha}_{2, j} z^{-(i+j)}
$$

This condition is satisfied for

$$
c_{k}=\sum_{i=\max (0, k-(M-1))}^{\min (k, M-1)} \hat{\alpha}_{1, i} \hat{\alpha}_{2, k-i}
$$

The frequency responses of the cross-talk pre-cancellation filters $A_{11}, A_{12}, A_{21}$ and $A_{22}$ can be realized using IIR filters or approximated as FIR filters.

\subsection{Measurement setup}

The indirect learning architectures for predistrortion as shown in Fig. 3.1-Fig. 3.4 require an update of the DPD coefficients every sample duration. This will lead to prohibitively complex hardware requirements. So, we choose a block adaptive approach, where blocks of $N$ samples representing the input signal are used to excite the PA. As there is no information about the PA response available to construct a $\mathrm{DPD}$, the input to the PA is not a predistorted signal during the first block. The DPD coefficients are computed from the input and output signal blocks. From the second block onward the predistorted signal is used to excite the PA, and the DPD coefficients are updated every block. The estimation of predistorter coefficients in the case of CTC-DPD requires $\boldsymbol{v}_{1}$ and $\boldsymbol{v}_{2}$ according to (3.14), which are not available during the first block. However the DPD coefficients can be estimated using $\boldsymbol{u}_{1}$ and $\boldsymbol{u}_{2}$ which can be constructed from $\boldsymbol{x}_{1}, \boldsymbol{x}_{2}$ and the estimates of the coupling coefficients.

The measurement setup is shown in Fig. 3.7. The base-band transmit signals are generated and the cross-talk is applied in a computer. Data blocks of $N$ samples, which represent these signals are sent to the Rohde \& Schwarz SMU-200A signal generator. The RF signals of the two ports of the signal generator, which emulate the MIMO transmitter, are fed to the input ports of the power amplifiers. We use 


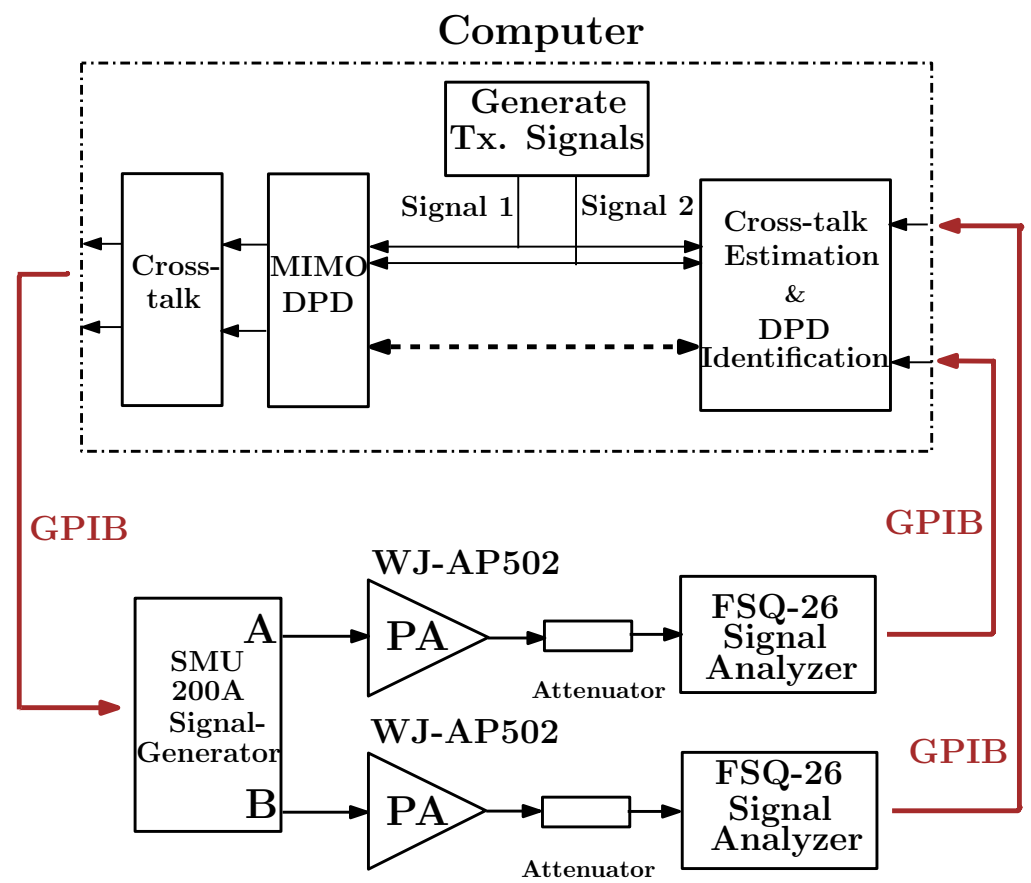

Fig. 3.7: The measurement setup

InGaP HBT power amplifiers AP-502 operating at $2140 \mathrm{MHz}$, from WJ Communications Inc. The PA output signals are captured using Rohde \& Schwarz FSQ-26 signal analyzers, and the input and output signals are synchronized offline before the estimation of the coupling factors which model the cross-talk, and the DPD coefficients.

The minimum required sampling rate for an accurate representation and thus compensation of $n^{\text {th }}$ order intermodulation products is equal to $n$ times the RF bandwidth of the input signal [52]. As we use a $9^{\text {th }}$ order memory polynomial model predistorter in our measurements, the sampling rate of the input and output signals are chosen to be 10 times the input signal bandwidth.

The attenuation factors $G_{1}$ and $G_{2}$ applied to the output signals are chosen as the ratio of maximum envelope values of the PA output and the input signals [53]. The signal generator and analyzer communicate with the computer using general-purpose interface bus (GPIB). This process is repeated 5 more times with the predistorted signals. The peak envelope power of the PA output signal $\left(\mathrm{PEP}_{\text {out }}\right)$ is kept constant by fixing the peak envelope power of the $\mathrm{PA}$ input signal $\left(\mathrm{PEP}_{\text {in }}\right)$ for all the 6 blocks transmitted. This is done so that all the signals used in the measurements experience similar AM-AM response. We use the notation $k=1,2, \ldots 6$ to denote these blocks in the next section. Finally this process is repeated with C-DPD, CO-DPD and CTC-DPD for different values of $\mathrm{PEP}_{\text {in }}$, different levels of frequency flat/selective cross-talk and different transmit signals. Further details of the measurements are given in Table 3.1 and some details regarding the transmit signals are given in 


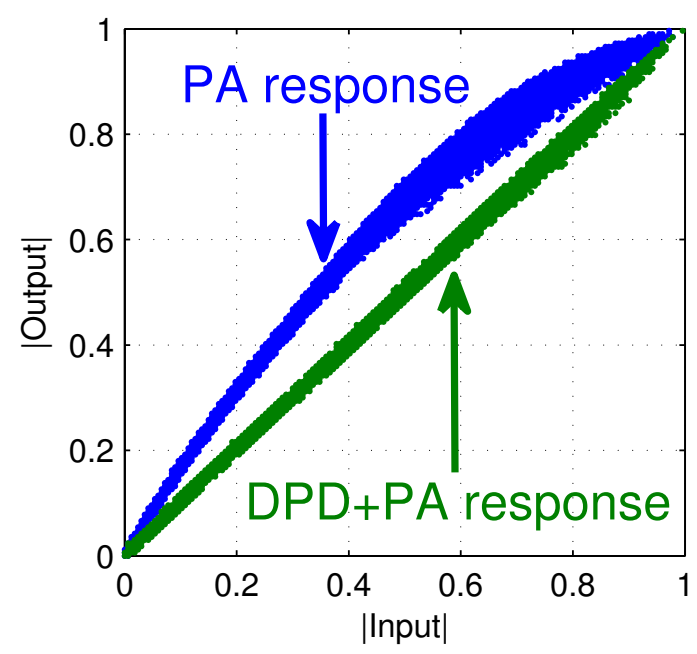

(a) $\mathrm{PEP}_{\text {in }}=7 \mathrm{dBm}$

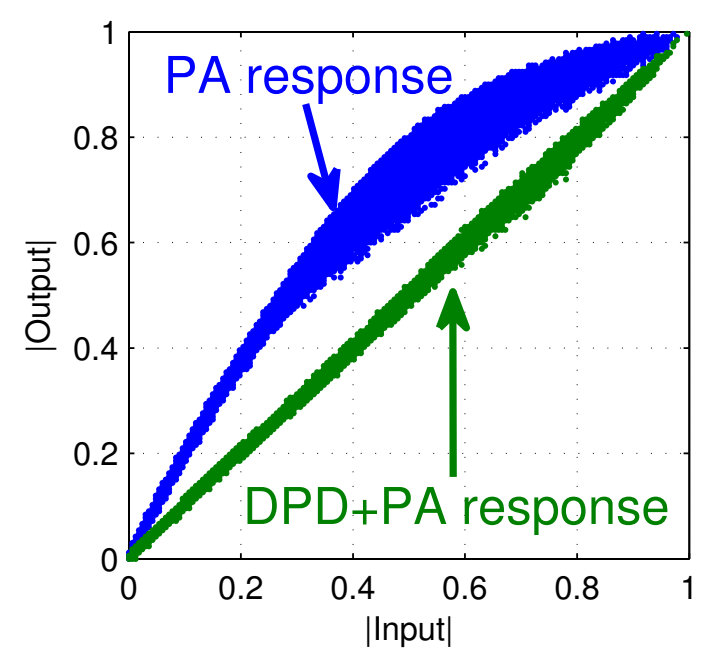

(b) $\mathrm{PEP}_{\text {in }}=10 \mathrm{dBm}$

Fig. 3.8: AM-AM plots (signal E)

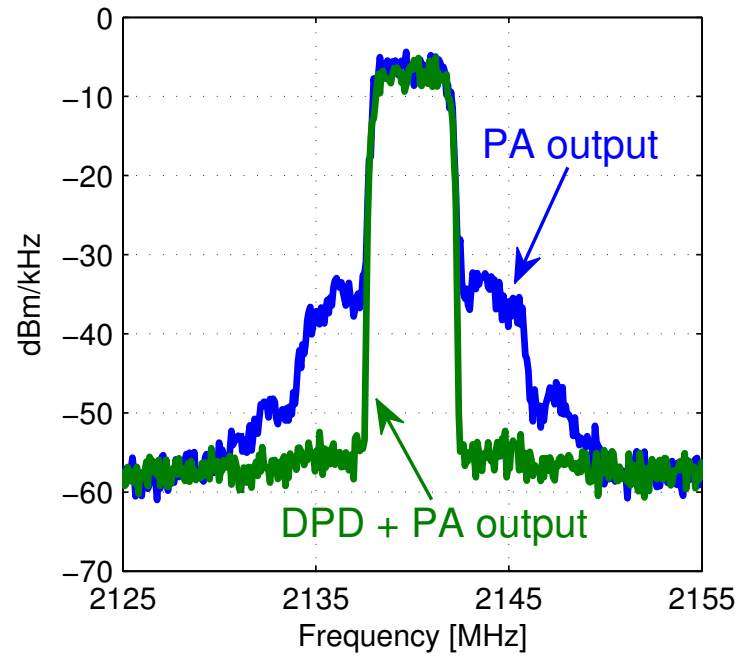

(a) $\mathrm{PEP}_{\text {in }}=7 \mathrm{dBm}$

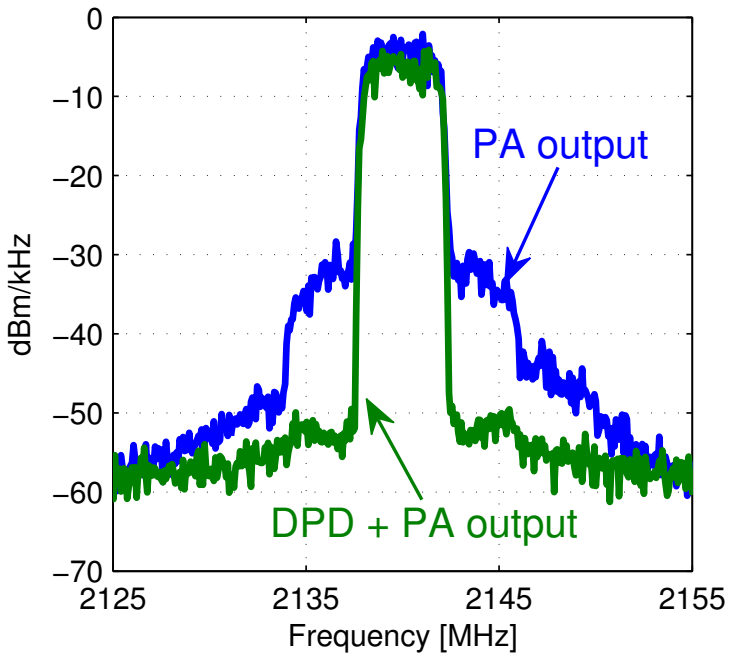

(b) $\mathrm{PEP}_{\text {in }}=10 \mathrm{dBm}$

Fig. 3.9: PSD spectra (signal E) 
Table 3.1.: Details of the measurements

\begin{tabular}{|c|c|c|}
\hline \multicolumn{2}{|c|}{ Power amplifier (PA) } & WJ-AP502 \\
\hline \multicolumn{2}{|l|}{ Supply voltage } & $7 \mathrm{~V}$ \\
\hline \multicolumn{2}{|l|}{ Bias voltage } & $3.6 \mathrm{~V}$ \\
\hline \multicolumn{2}{|l|}{ Center frequency } & $2140 \mathrm{MHz}$ \\
\hline \multicolumn{2}{|l|}{ Signal } & Rohde \& Schwarz \\
\hline \multicolumn{2}{|l|}{ Generator } & SMU-200A \\
\hline \multicolumn{2}{|l|}{ Signal } & Rohde \& Schwarz \\
\hline \multicolumn{2}{|l|}{ Analyzer } & FSQ-26 \\
\hline \multicolumn{2}{|l|}{ Number of samples } & 153600 \\
\hline \multirow{2}{*}{\multicolumn{2}{|c|}{$\begin{array}{c}\text { in a block } \\
(N)\end{array}$}} & (QPSK,16-QAM,UMTS) \\
\hline & & 150000 (LTE) \\
\hline \multirow{2}{*}{$\begin{array}{l}\text { Memory polynomial } \\
\text { for DPD }\end{array}$} & $K$ & 9 \\
\hline & $Q$ & 3 \\
\hline
\end{tabular}

Table 3.2. The threshold value for the estimation of the coupling factors is chosen as $T=0.3$ in all our measurements.

The AM-AM plots and the power spectral densities of the PA output signals, corresponding to $\mathrm{PEP}_{\text {in }}=7 \mathrm{dBm}$ and $\mathrm{PEP}_{\text {in }}=10 \mathrm{dBm}$, for signal E are shown in Fig. 3.8 and Fig. 3.9 respectively. The transmit signals (A-G) considered in our study include QPSK, 16-QAM, UMTS and LTE signals with different bandwidths, pulse-shaping filters and PAPRs. The PDFs of the input signal magnitudes are shown in Fig. 3.10. The QPSK and 16-QAM signals are generated by upsampling the symbols, which have a symbol-rate of $1 \mathrm{MHz}$ by a factor $R=10$ and filtering using RRC filters. The bandwidth of the QPSK/16-QAM signals is $1 \mathrm{MHz}$ and the out of band roll-off depends on the roll-off factor of the pulse-shaping RRC filter. A block of $N$ samples contains 15360 QPSK/16-QAM symbols. The UMTS signals are generated by up-sampling the spread data sequences which are at a chip rate of $3.84 \mathrm{MHz}$ by a factor $R=10$ and filtering using a root raised cosine (RRC) filter [54]. A block of $N$ samples of the UMTS signal contains 15360 chips. The details of the data and control channels in the UMTS signals are given in Table 3.3. DPCCH (Dedicated Physical Control Channel), DPDCH (Dedicated Physical Data Channel), HSDPCCH (High Speed-Dedicated Physical Control Channel), E-DPCCH (EnhancedDedicated Physical Control Channel) and E-DPDCH (Enhanced-Dedicated Physical Data Channel) denote data and control channels in UMTS [54]. $N_{c h}, S F$ and $\beta$ represent the number of channels, spreading factor and weight factors, respectively. The LTE signal of bandwidth $10 \mathrm{MHz}$ is generated by mapping QPSK symbols on to 600 sub-carriers using single carrier frequency division multiplexing (SC-FDM) [55]. A 


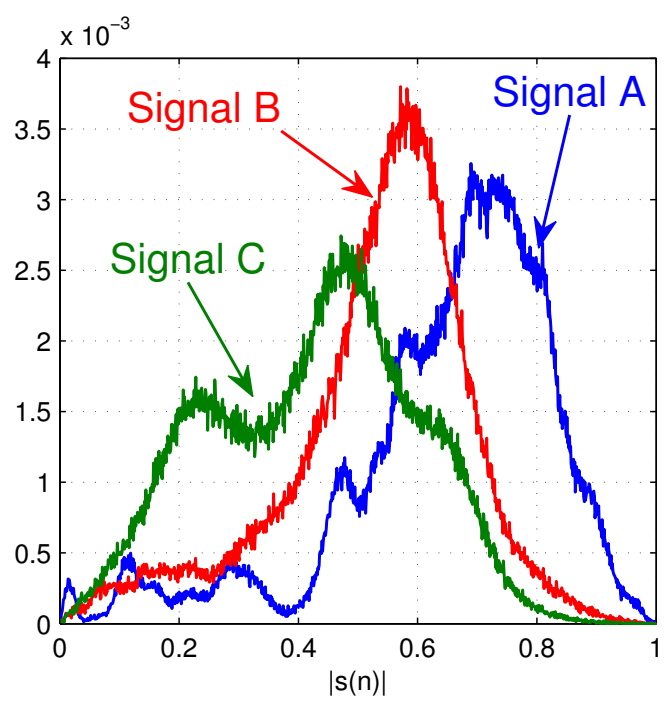

(a) QPSK and 16-QAM

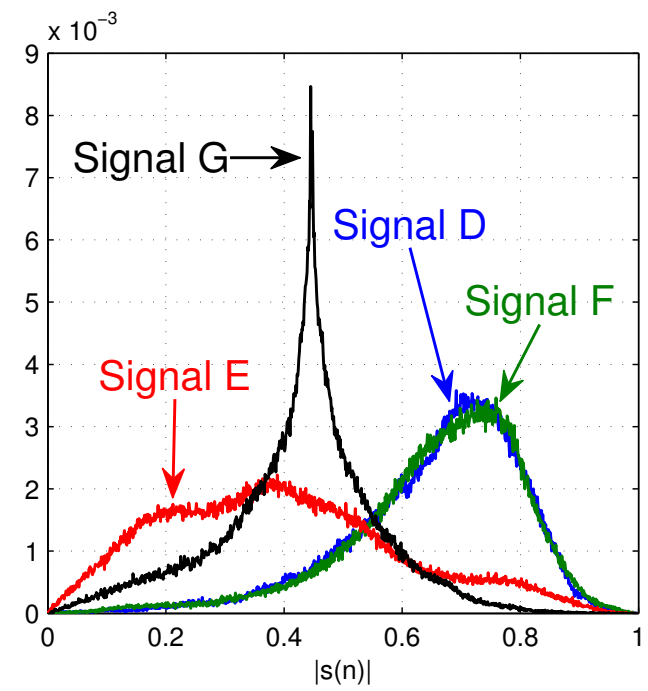

(b) UMTS and LTE

Fig. 3.10: Empirical PDF of the input signal magnitude

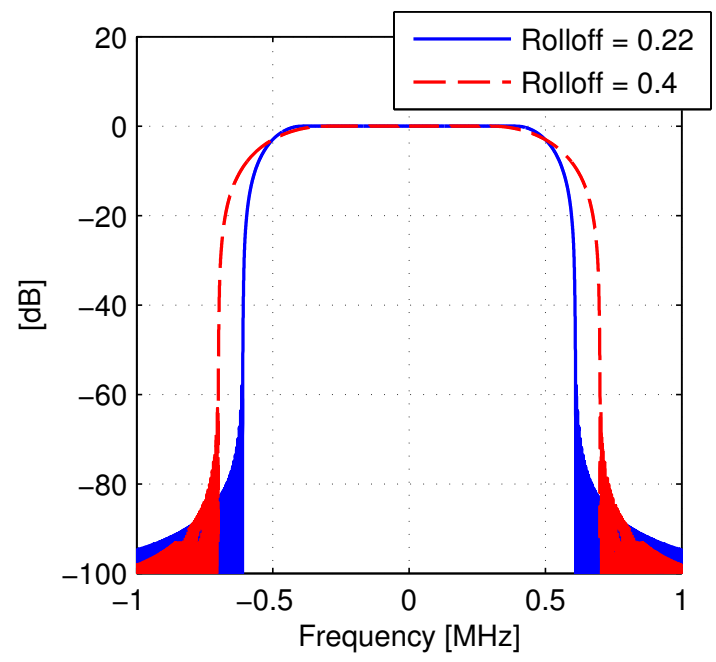

(a) QPSK and 16-QAM

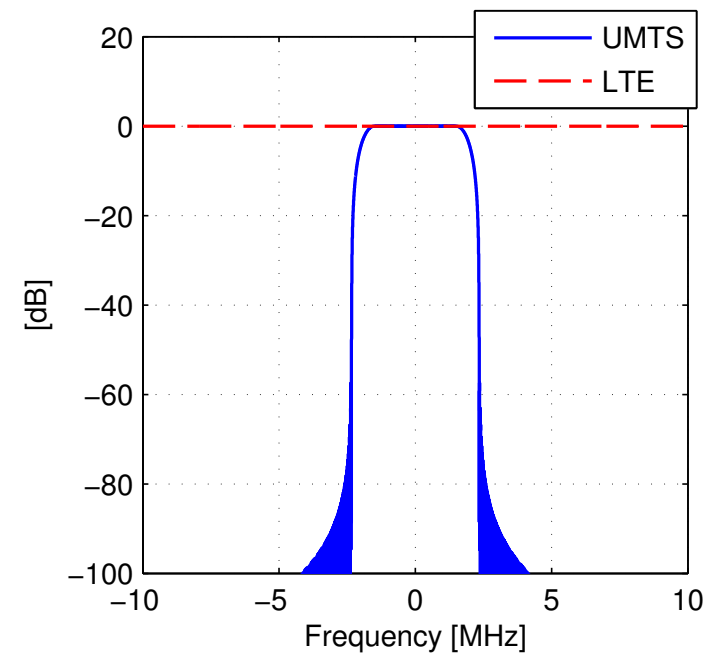

(b) UMTS and LTE

Fig. 3.11: Pulse shaping filter response $|P(f)|$ 
block of $N$ samples of the LTE signal contains 21 SC-FDM symbols. The frequency responses of the pulse shaping filters are shown in Fig. 3.11.

\subsection{LInearization performance indicators}

The linearization performances of C-DPD, CO-DPD and CTC-DPD in a two-antenna transmitter are compared based on the following performance criteria: $\mathrm{NMSE}_{\mathrm{IB}}$, EVM, ACLR and ACEPR. We also introduce definitions of the error in the least squares estimation $\left(\varepsilon_{L S}\right)$ and residual leakage $\left(\varepsilon_{L e a k}\right)$, which are suitable for our analysis. These performance measures are used to interpret the measurement results and support our arguments. The In-band Normalized Mean Squared Error $\left(\mathrm{NMSE}_{\mathrm{IB}}\right)$ between the input and output signals is [56]

$$
\begin{gathered}
\operatorname{nmse}_{\mathrm{IB}, \mathrm{i}}=\frac{\int\left|\left(S_{i}(f)-Y_{i}(f)\right) P(f)\right|^{2} d f}{\int\left|Y_{i}(f) P(f)\right|^{2} d f}, i=1,2 . \\
\mathrm{NMSE}_{\mathrm{IB}}=10 \log \left\{\frac{1}{2}\left(\mathrm{nmse}_{\mathrm{IB}, 1}+\mathrm{nmse}_{\mathrm{IB}, 2}\right)\right\}
\end{gathered}
$$

where $S_{i}(f), Y_{i}(f)$ and $P(f)$ are the discrete time Fourier transforms of the input signal $s_{i}(n)$, output signal $y_{i}(n)$ and the impulse response of the pulse shaping filter $p(n)$ respectively and $f$ is the normalized frequency. The error vector magnitude (EVM) is computed as follows: First, the input and output signals are filtered with $p(n)$

$$
s_{p, i}(n)=s_{i}(n) * p(n), y_{p, i}(n)=y_{i}(n) * p(n) .
$$

Then, in the case of QAM and UMTS, these filtered signals are down-sampled by a factor $R=10$.

$$
s_{\text {sym }, i}(n)=s_{p, i}(R n), \quad y_{\text {sym }, i}(n)=y_{p, i}(R n)
$$

These are the sequences of symbols for QPSK and 16-QAM and sequences of chips for UMTS. For the LTE signal, SC-FDM demodulation [55] is performed on the filtered signals to obtain the input and output symbols $s_{\text {sym }, i}$ and $y_{s y m, i}$. These symbols are packed together to form input and output symbol vectors $\boldsymbol{s}_{\text {sym }, i}$ and $\boldsymbol{y}_{\text {sym }, i}$. Finally the EVM is computed as

$$
\begin{gathered}
\mathrm{EVM}_{\mathrm{i}}=\frac{\left\|\boldsymbol{s}_{\text {sym }, i}-\boldsymbol{y}_{\text {sym }, i}\right\|}{\left\|\boldsymbol{s}_{\text {sym }, i}\right\|} \times 100 \% \\
\mathrm{EVM}=\frac{1}{2}\left(\mathrm{EVM}_{1}+\mathrm{EVM}_{2}\right) .
\end{gathered}
$$


Table 3.2.: Details of the Transmit signals

\begin{tabular}{c|c|c|c|c|c}
\hline $\begin{array}{c}\text { Tx. } \\
\text { Signal }\end{array}$ & Type & $\begin{array}{c}\text { PAPR } \\
{[\mathrm{dB}]}\end{array}$ & $\begin{array}{c}\text { Rolloff- } \\
\text { factor }(r)\end{array}$ & $\begin{array}{c}\text { BW } \\
{[\mathrm{MHz}]}\end{array}$ & $\begin{array}{c}F_{s} \\
{[\mathrm{MHz}]}\end{array}$ \\
\hline \hline A & QPSK & 3.4 & 0.40 & 1 & 10 \\
\hline B & QPSK & 5.1 & 0.22 & 1 & 10 \\
\hline C & 16-QAM & 6.8 & 0.22 & 1 & 10 \\
\hline D & UMTS & 3.5 & 0.22 & 3.84 & 38.4 \\
\hline E & UMTS & 7.2 & 0.22 & 3.84 & 38.4 \\
\hline F & UMTS & 3.4 & 0.22 & 3.84 & 38.4 \\
\hline G & LTE & 6.9 & NA & 10 & 100 \\
\hline
\end{tabular}

Table 3.3.: Details of UMTS signals

\begin{tabular}{c|c|c|c|c}
\hline \multicolumn{2}{c|}{} & Signal D & Signal E & Signal F \\
\hline \hline \multirow{4}{*}{ DPCCH } & $N_{c h}$ & 1 & 1 & 1 \\
\cline { 2 - 5 } & $S F$ & 256 & 256 & 256 \\
\cline { 2 - 5 } & $\beta_{c}$ & 0.5333 & 1 & 0.1333 \\
\hline \multirow{4}{*}{ DPDCH } & $N_{c h}$ & 1 & 0 & 1 \\
\cline { 2 - 5 } & $S F$ & 64 & - & 64 \\
\cline { 2 - 5 } & $\beta_{d}$ & 1 & - & 1 \\
\hline \multirow{4}{*}{ ES-DPCCH } & $N_{c h}$ & 0 & 1 & 1 \\
\cline { 2 - 5 } & $S F$ & - & 256 & 256 \\
\cline { 2 - 5 } & $\beta_{h s}$ & - & 1 & 0.2667 \\
\hline \multirow{5}{*}{ E-DPDCH } & $N_{c h}$ & 0 & 1 & 0 \\
\cline { 2 - 5 } & $S F$ & - & 256 & - \\
\cline { 2 - 5 } & $\beta_{e c}$ & - & 0.5333 & - \\
\cline { 2 - 5 } & $N_{c h}$ & 0 & 4 & - \\
\cline { 2 - 5 } & $S F$ & - & {$[224$} & 4 \\
\cline { 2 - 5 } & $\beta_{e d}$ & - & $0.80 .60 .6]$ & - \\
\hline
\end{tabular}


The Adjacent Channel Leakage Ratio (ACLR) is defined as

$$
\begin{gathered}
\operatorname{aclr}_{\text {upper }, \mathrm{i}}=\frac{\int\left|Y_{i}(f) P(f)\right|^{2} d f}{\int\left|Y_{i}(f) P\left(f-f_{a d j}\right)\right|^{2} d f} \\
\operatorname{aclr}_{\text {lower }, \mathrm{i}}=\frac{\int\left|Y_{i}(f) P(f)\right|^{2} d f}{\int\left|Y_{i}(f) P\left(f+f_{a d j}\right)\right|^{2} d f} \\
\mathrm{ACLR}=10 \log \left\{\frac { 1 } { 4 } \left(\operatorname{aclr}_{\text {lower }, 1}+\operatorname{aclr}_{\text {lower }, 2}\right.\right. \\
\left.\left.+\operatorname{aclr}_{\text {upper }, 1}+\operatorname{aclr}_{\text {upper }, 2}\right)\right\} .
\end{gathered}
$$

The Adjacent Channel Error Power Ratio (ACEPR) is defined as [56]

$$
\begin{aligned}
\operatorname{acepr}_{\text {upper }, \mathrm{i}} & =\frac{\int_{-\infty}^{\infty}\left|\left(s_{i}(f)-y_{i}(f)\right) w\left(f-f_{\text {adj }}\right)\right|^{2} d f}{\int_{-\infty}^{\infty}\left|s_{i}(f) w(f)\right|^{2} d f} \\
\operatorname{acepr}_{\text {lower }, \mathrm{i}} & =\frac{\int_{-\infty}^{\infty}\left|\left(s_{i}(f)-y_{i}(f)\right) w\left(f+f_{\text {adj }}\right)\right|^{2} d f}{\int_{-\infty}^{\infty}\left|s_{i}(f) w(f)\right|^{2} d f} \\
\mathrm{ACEPR} & =10 \log \left\{\frac { 1 } { 4 } \left(\operatorname{acepr}_{\text {lower }, 1}+\text { acepr }_{\text {lower }, 2}\right.\right. \\
& \left.\left.+ \text { acepr }_{\text {upper }, 1}+\operatorname{acepr}_{\text {upper }, 2}\right)\right\}
\end{aligned}
$$

where $f_{a d j}$ is the frequency separation between the desired channel where the signal is present and the adjacent channel. $f_{a d j}=(1+r) \times 1 \mathrm{MHz}$ for QAM signals (where $r$ is the roll-off factor of the pulse shaping filter), $5 \mathrm{MHz}$ for UMTS signals and $10 \mathrm{MHz}$ for the LTE signal. The EVM and ACLR computations for UMTS and LTE signals are as described in [57] and [58], respectively.

The average energy in the error signals associated with the LS estimation of the post-distorter coefficients ${ }^{3}$ for a two-antenna transmitter can be defined as

$$
\varepsilon_{L S}=10 \log \left\{\frac{1}{2}\left(\left\|\boldsymbol{e}_{1}\right\|^{2}+\left\|\boldsymbol{e}_{2}\right\|^{2}\right)\right\}
$$

where

$$
\begin{aligned}
& \boldsymbol{e}_{1}=\boldsymbol{x}_{1}-\hat{\boldsymbol{x}}_{1}, \quad \boldsymbol{e}_{2}=\boldsymbol{x}_{2}-\hat{\boldsymbol{x}}_{2} \text { for CO-DPD } \\
& \boldsymbol{e}_{1}=\boldsymbol{v}_{1}-\hat{\boldsymbol{v}}_{1}, \quad \boldsymbol{e}_{2}=\boldsymbol{v}_{2}-\hat{\boldsymbol{v}}_{2} \text { for CTC-DPD }
\end{aligned}
$$

and $\|$.$\| represents the L_{2}$ norm of a vector. $\varepsilon_{L S}$ depends on the signal characteristics, PA response, and the accuracy of the MIMO-DPD in compensating the cross-talk and PA non-linearity as a post-distorter.

The residual leakage associated with a DPD structure for a MIMO transmitter is defined as the signal component present in the complex envelope of the input signal to one of the PAs from the input signals of the other paths (e.g., $s_{2}(n)$ in $u_{1}(n)$ ). The dotted lines in Fig. 3.12 and Fig. 3.13 show the signal paths that create the

\footnotetext{
${ }^{3}$ The post-distorter is used as the predistorter in indirect learning architecture
} 


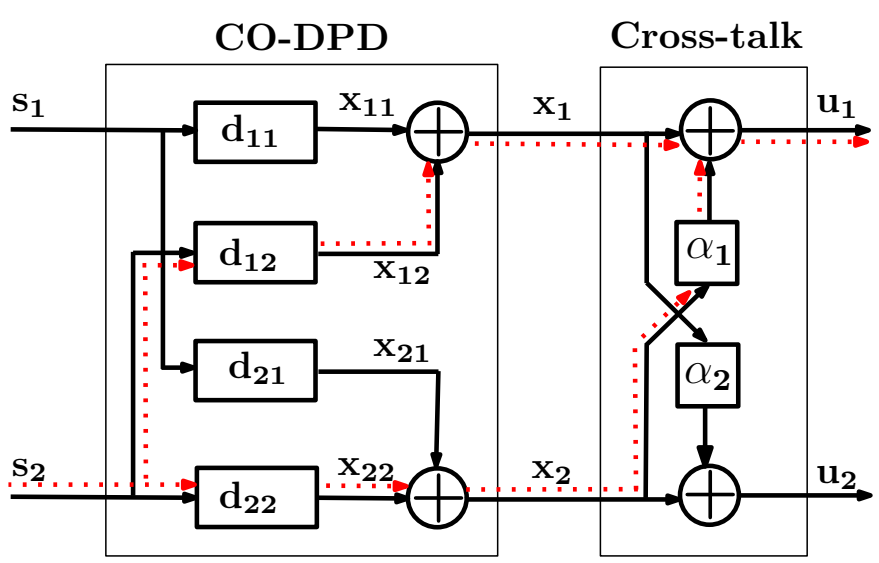

Fig. 3.12: Residual leakage with CO-DPD

residual leakage in the first path due to the input signal in the second path, in a two-antenna transmitter employing CO-DPD and CTC-DPD respectively. The signal components in CO-DPD as shown in Fig. 3.12 can be expressed as

$$
\begin{array}{ll}
\boldsymbol{x}_{11}=\boldsymbol{A}_{s_{1}} \boldsymbol{d}_{11}, & \boldsymbol{x}_{12}=\boldsymbol{A}_{s_{2}} \boldsymbol{d}_{12} \\
\boldsymbol{x}_{22}=\boldsymbol{A}_{s_{2}} \boldsymbol{d}_{22}, & \boldsymbol{x}_{21}=\boldsymbol{A}_{s_{1}} \boldsymbol{d}_{21}
\end{array}
$$

The DPD output signals in CTC-DPD are

$$
\begin{aligned}
& \boldsymbol{v}_{1}=\boldsymbol{A}_{s_{1}} \boldsymbol{d}_{1} \\
& \boldsymbol{v}_{2}=\boldsymbol{A}_{s_{2}} \boldsymbol{d}_{2}
\end{aligned}
$$

The signal components in CTC-DPD, for frequency flat cross-talk are

$$
\begin{array}{lll}
\boldsymbol{x}_{11}=\frac{1}{1-\hat{\alpha}_{1} \hat{\alpha}_{2}} \boldsymbol{v}_{1}, & \boldsymbol{x}_{12}=\frac{-\hat{\alpha}_{1}}{1-\hat{\alpha}_{1} \hat{\alpha}_{2}} \boldsymbol{v}_{2} \\
\boldsymbol{x}_{22}=\frac{1}{1-\hat{\alpha}_{1} \hat{\alpha}_{2}} \boldsymbol{v}_{2}, & \boldsymbol{x}_{21}=\frac{-\hat{\alpha}_{2}}{1-\hat{\alpha}_{1} \hat{\alpha}_{2}} \boldsymbol{v}_{1}
\end{array}
$$

and for frequency selective cross-talk

$$
\begin{array}{ll}
\boldsymbol{x}_{11}=x_{1}(n) * a_{11}(n), & \boldsymbol{x}_{12}=x_{2}(n) * a_{12}(n) \\
\boldsymbol{x}_{22}=x_{2}(n) * a_{22}(n), & \boldsymbol{x}_{21}=x_{1}(n) * a_{21}(n)
\end{array}
$$

where $a_{i j}(n)$ is the inverse $z$-transform of $A_{i j}(z)$ for $i, j \in 1,2$. The residual leakage signals for both the MIMO-DPDs, in the case of frequency flat cross-talk are

$$
\begin{aligned}
& \boldsymbol{u}_{\text {leak }, 1}=\boldsymbol{x}_{12}+\alpha_{1} \boldsymbol{x}_{22} \\
& \boldsymbol{u}_{\text {leak }, 2}=\boldsymbol{x}_{21}+\alpha_{2} \boldsymbol{x}_{11}
\end{aligned}
$$

and in the case of frequency selective cross-talk

$$
\begin{aligned}
\boldsymbol{u}_{\text {leak }, 1} & =\boldsymbol{x}_{12}+\sum_{j=0}^{M-1} \alpha_{1, j} \boldsymbol{x}_{22}(n-j) \\
\boldsymbol{u}_{\text {leak }, 2} & =\boldsymbol{x}_{21}+\sum_{j=0}^{M-1} \alpha_{2, j} \boldsymbol{x}_{11}(n-j) .
\end{aligned}
$$




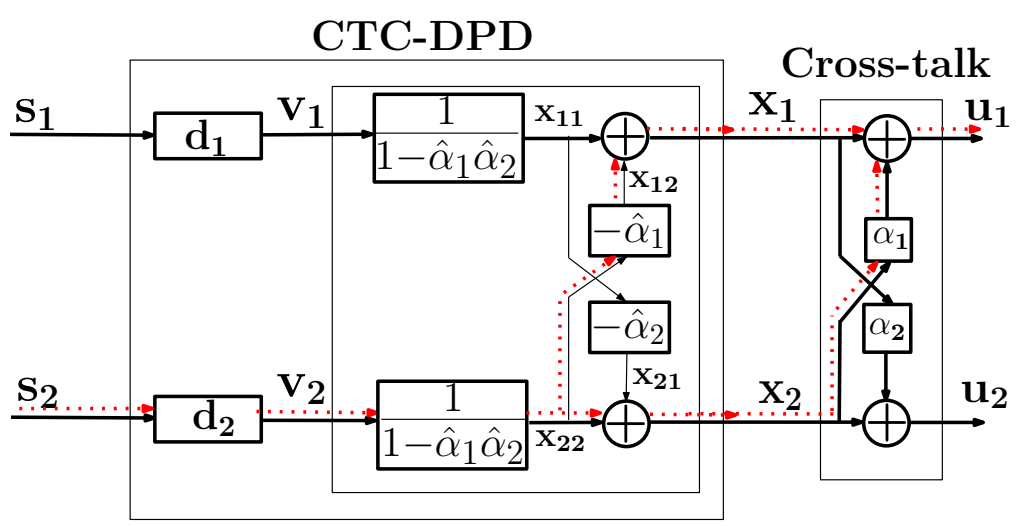

Fig. 3.13: Residual leakage with CTC-DPD

The average energy in the residual leakage signals is

$$
\varepsilon_{\text {Leak }}=10 \log \left\{\frac{1}{2}\left(\left\|\boldsymbol{u}_{\text {leak }, 1}\right\|^{2}+\left\|\boldsymbol{u}_{\text {leak }, 2}\right\|^{2}\right)\right\}
$$

The cross-over coefficients are estimated to minimize the error between the inputs to the MIMO transmitter and the outputs of the post-distorters in the least square sense. So, the use of these coefficients as a MIMO-DPD will invariably create some residual leakage. In the case of $\mathrm{CTC}-\mathrm{DPD}$, the error in the estimation of the coupling coefficients is solely responsible for the residual leakage, and the amount of residual leakage can be reduced by improving the quality of the estimation of the coupling coefficients.

\subsection{Measurement results and discussion}

Fig. 3.14 shows the EVM and ACLR averaged over the blocks $k=3 \ldots 6$, for CDPD and the MIMO-DPDs for different levels of frequency flat cross-talk. The performance of the C-DPD degrades as the cross-talk level increases, whereas the CO-DPD and CTC-DPD performances are unaffected by the cross-talk level. The linearization performances of MIMO-DPDs in the presence of frequency-flat and frequency-selective cross-talks are discussed in detail in section 3.5.1 and section 3.5.2 respectively. The measurements were carried out in a block-by-block manner as described in section 3.3. Only the results for $k=2,3 . .6$ are presented here, as $k=1$ is without predistortion.

As the input to the PA is not a predistorted signal during the the first block and a predistorted signal for subsequent blocks, there is a difference in the input signal characteristics between the blocks $k=1$ and $k=2$. The DPD usually expands the signal values with higher magnitude and compresses those with lower magnitude, 


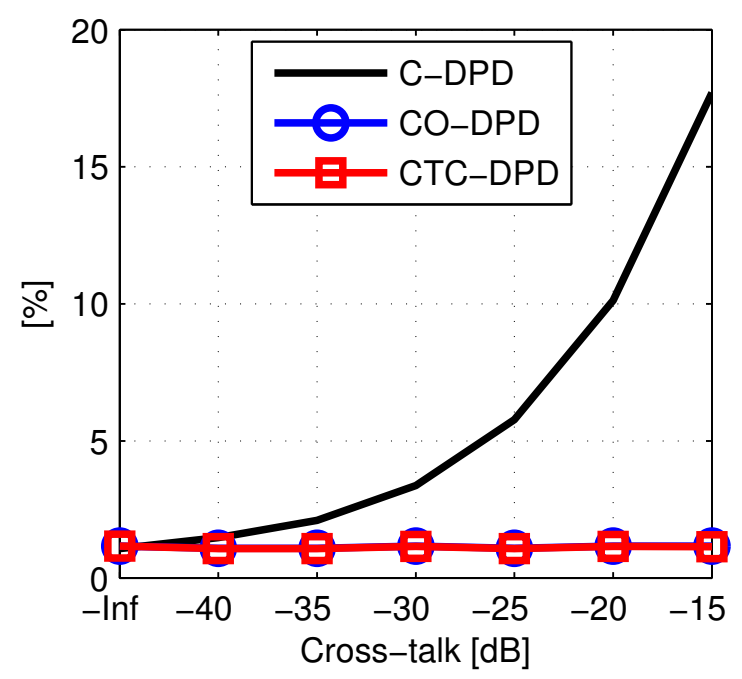

(a) EVM

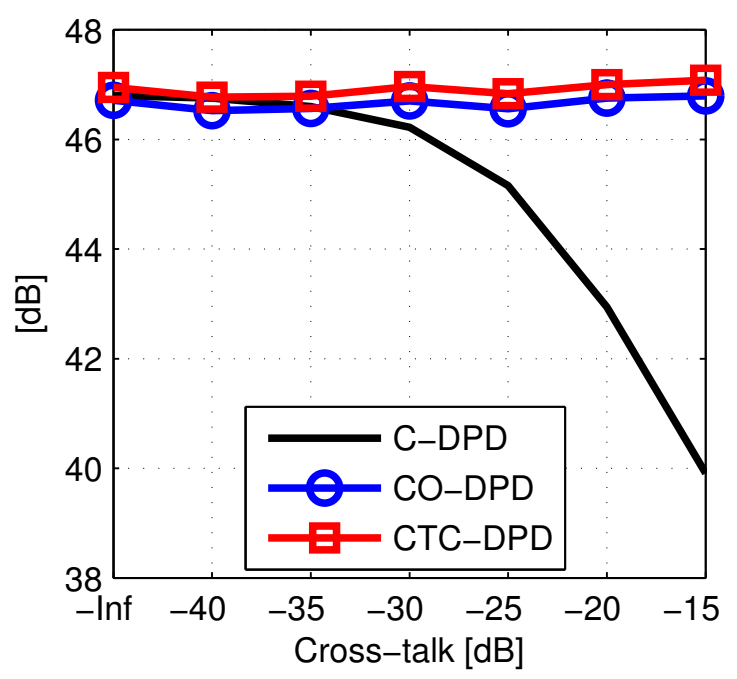

(b) ACLR

Fig. 3.14: Comparison of C-DPD, CO-DPD and CTC-DPD, signal E, $\mathrm{PEP}_{\text {in }}=$ $7 \mathrm{dBm}$

as it tries to invert the PA behavior, which compresses the signal in the higher magnitude region. This means that the PAPR of predistorted signal is higher than that of the input signal. In our measurements, we keep the $\mathrm{PEP}_{\text {out }}$ the same for all the blocks $(k=1,2, \ldots 6)$ by fixing $\mathrm{PEP}_{\text {in }}$. As the PAPR of $x(n)$ is larger for the blocks $k=2,3 . .6$ compared to $k=1$, the average input and output powers are lower for $k=2,3 . .6$ compared to $k=1$. This average power mismatch and the differences and input signal characteristics [59,60] between the blocks $k=1$ and $k=2$ result in a slight degradation of linearization performance for the second block compared to the subsequent blocks.

$\mathrm{NMSE}_{\mathrm{IB}}, \mathrm{ACLR}, \mathrm{EVM}$ and $\varepsilon_{\text {Leak }}$ (for $k=2 \ldots 6$ ) are the quantities directly related to the linearization performance and $\varepsilon_{L S}$ (for $k=1 \ldots 5$ ) is a measure of the accuracy of DPD estimation. As $\mathrm{NMSE}_{\mathrm{IB}}$, ACLR, EVM and $\varepsilon_{\text {Leak }}$ are approximately unchanging for $k=3 \ldots 6$, we present their values at $k=2$ and the average value for $k=3 \ldots 6$. Similarly $\varepsilon_{L S}$ is approximately unchanging for $k=2 \ldots 5$, we present its values at $k=1$ and the average value for $k=2 \ldots 5$.

The differences in the performance measures between CO-DPD and CTC-DPD are

$$
\begin{gathered}
\Delta \mathrm{NMSE}_{\mathrm{IB}}=\mathrm{NMSE}_{\mathrm{IB}}(\mathrm{CO})-\mathrm{NMSE}_{\mathrm{IB}}(\mathrm{CTC}) \\
\Delta \mathrm{EVM}=\operatorname{EVM}(\mathrm{CO})-\operatorname{EVM}(\mathrm{CTC})
\end{gathered}
$$

$\Delta \mathrm{NMSE}_{\mathrm{IB}}$ and $\triangle \mathrm{EVM}$ are plotted against $n_{s c}$ in Fig. 3.15 , where $n_{s c}$ is used to index the transmitted signal and the cross-talk level jointly, according to

$$
n_{s c}=4\left(n_{s}-1\right)+n_{c}
$$




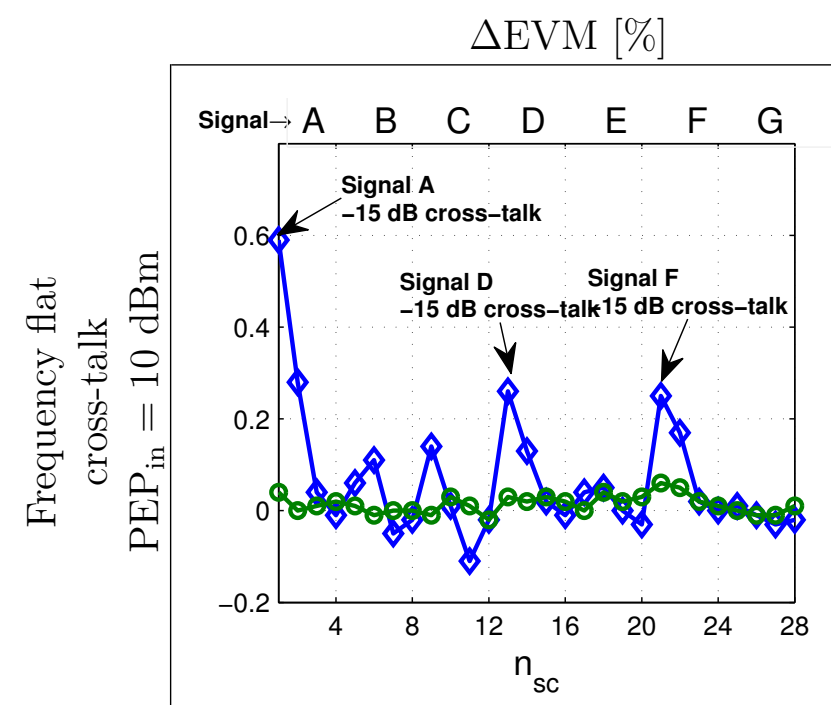

(a)
$\Delta \mathrm{NMSE}_{\mathrm{IB}}[\mathrm{dB}]$

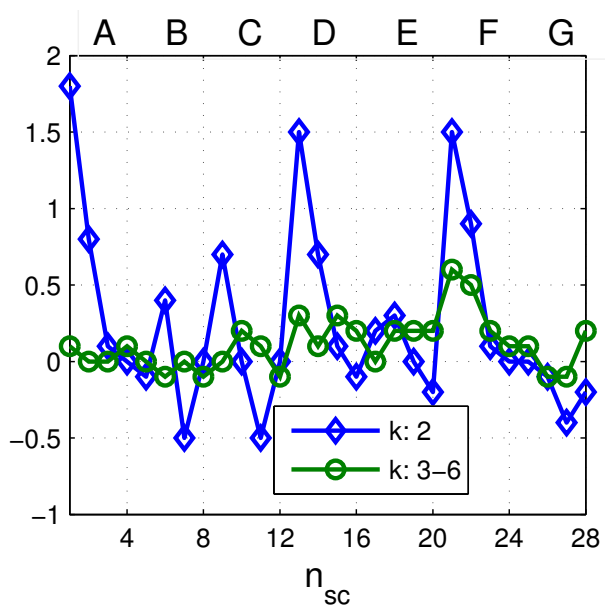

(b)

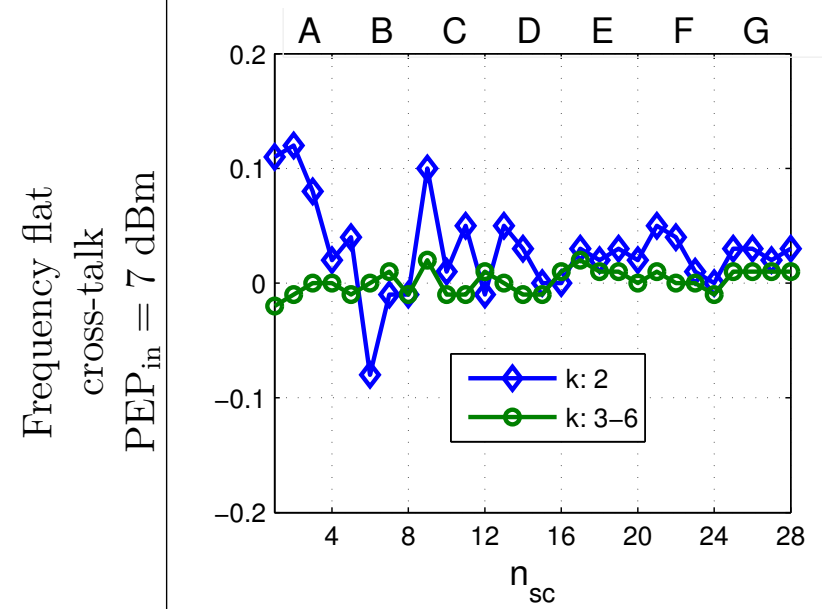

(c)

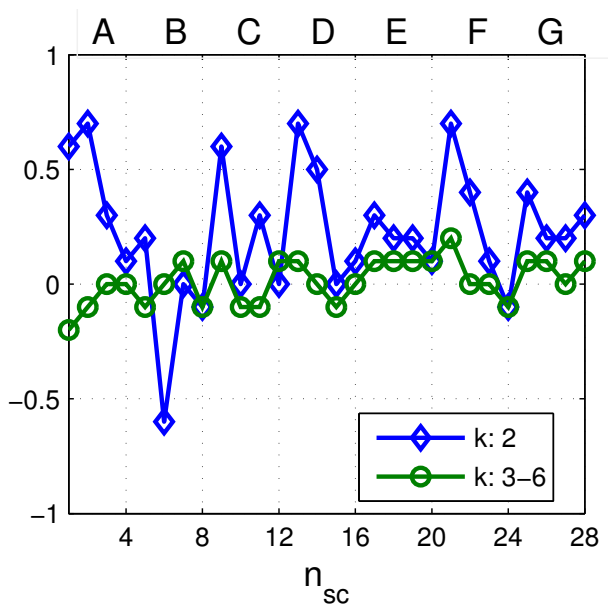

(d)

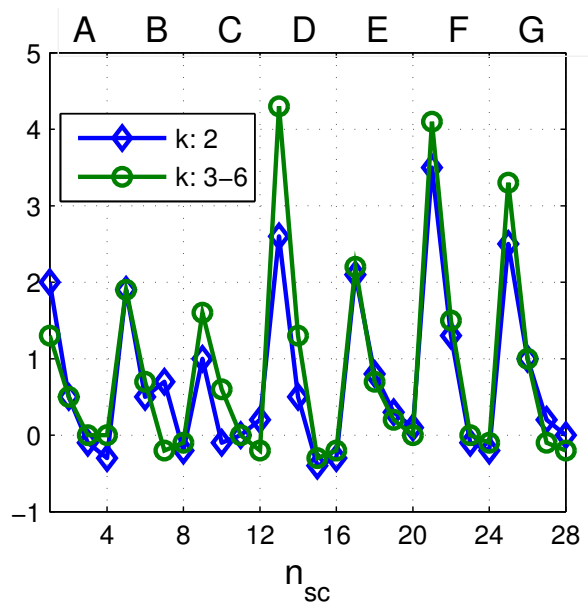

(f)

Fig. 3.15: Measurement results, $\Delta \mathrm{EVM}_{\mathrm{IB}}, \Delta \mathrm{NMSE}$ 


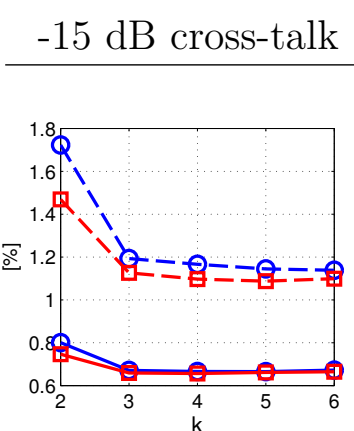

(a) EVM

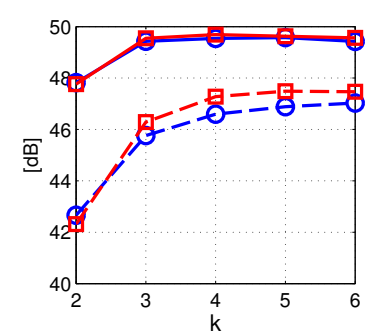

(e) ACLR

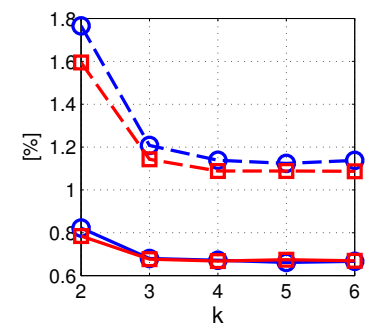

(b) EVM

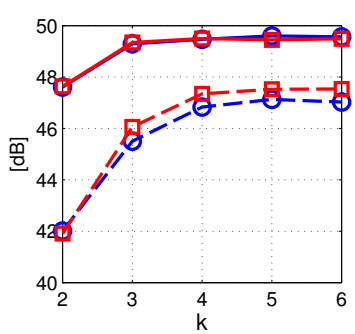

(f) ACLR

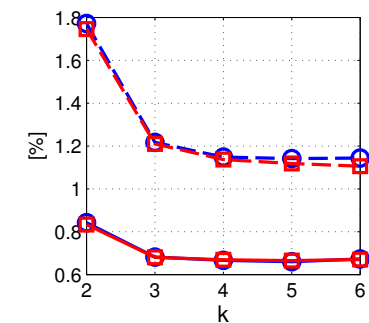

(c) EVM

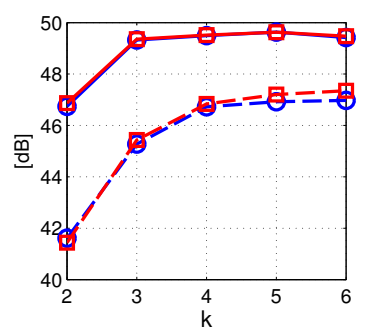

(g) ACLR

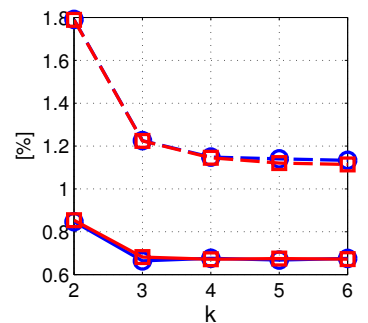

(d) EVM

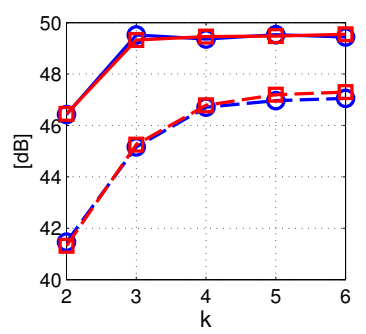

(h) ACLR

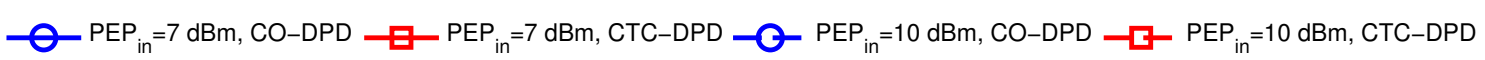

Fig. 3.16: Measurement results (EVM \& ACLR) for signal F, with frequency flat cross-talk

where $n_{s}$ is the signal index which is 1-7 for signal A-G, and $n_{c}$ is $1,2,3$ and 4 for crosstalk levels $-15 \mathrm{~dB},-20 \mathrm{~dB},-30 \mathrm{~dB}$ and the case without cross-talk respectively. $n_{s c}$ varies from 1 to 28 representing 7 signals at 4 different cross-talk levels. All the performance measures defined in this section are evaluated from our measurement data for different cases and presented in Appendix A.

\subsubsection{Frequency flat cross-talk}

The EVM and ACLR performances of CO-DPD and CTC-DPD at PEP in $=7 \mathrm{dBm}$ and $10 \mathrm{dBm}$ for signals $\mathrm{F}$ (UMTS signal with PAPR=3.4 dB) and E (UMTS signal with PAPR=7.2 dB) are shown in Fig. 3.16 and Fig. 3.17, respectively. Table 3.4 highlights the cases where CTC-DPD achieves a performance improvement over CODPD. At high cross-talk levels, CTC-DPD achieves a slight performance improvement compared to CO-DPD with signal $\mathrm{F}(\mathrm{PAPR}=3.4 \mathrm{~dB})$ at $\mathrm{PEP}_{\text {in }}=10 \mathrm{dBm}$. In this case, $\varepsilon_{\text {Leak }}$ is $-47.4 \mathrm{~dB}$ for CO-DPD and $-82.4 \mathrm{~dB}$ for CTC-DPD. This difference in $\varepsilon_{\text {Leak }}$ causes the difference in EVM between CO-DPD and CTC-DPD at $k=2$. For $k>2, \varepsilon_{\text {Leak }}$ is very low for both CO-DPD and CTC-DPD and their EVM performances are almost identical. For the other cases, the linearization performances of CO-DPD and CTC-DPD are very close. For these cases $\varepsilon_{\text {Leak }}$ is 


\section{$-15 \mathrm{~dB}$ cross-talk $\quad-20 \mathrm{~dB}$ cross-talk $\quad-30 \mathrm{~dB}$ cross-talk $\quad$ No cross-talk}

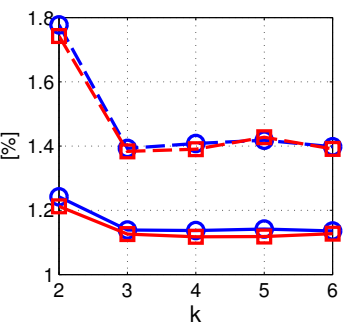

(a) EVM

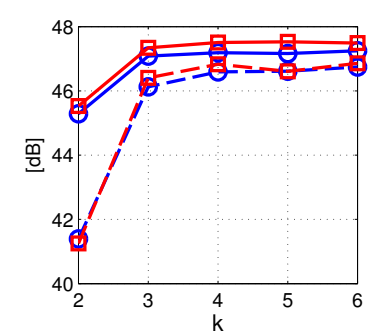

(e) ACLR

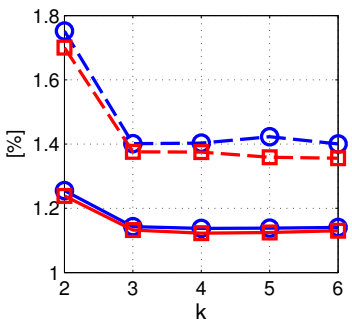

(b) EVM

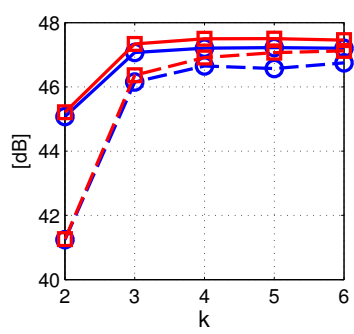

(f) ACLR

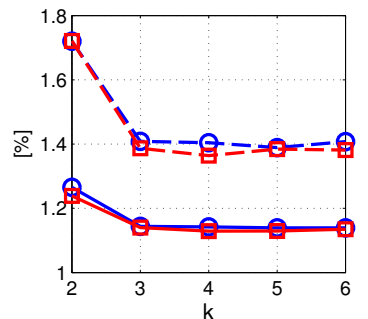

(c) EVM

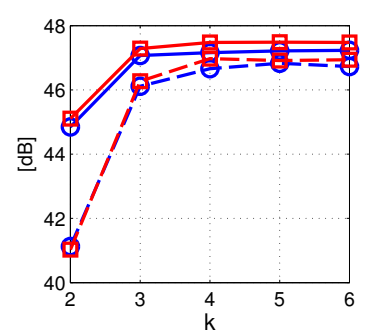

(g) ACLR

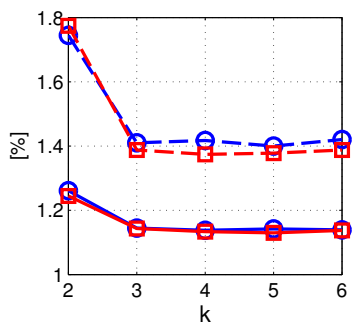

(d) EVM

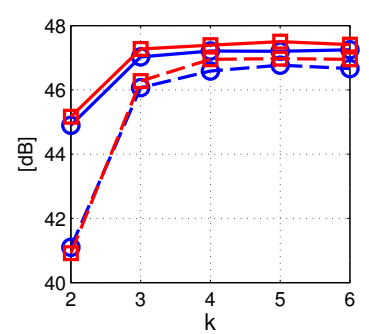

(h) ACLR

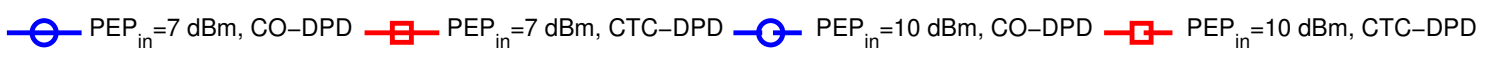

Fig. 3.17: Measurement results (EVM \& ACLR) for signal E with frequency flat cross-talk

very low for both CO-DPD and CTC-DPD as given in Table 3.4. Fig. 3.15 shows that CTC-DPD performs better only with signals A, D and F-which are all signals with low PAPR - at PEP in $=10 \mathrm{dBm}$, at high cross-talk levels (signals $\mathrm{A}, \mathrm{D}$ and $\mathrm{F}$ at $-15 \mathrm{~dB}$ cross-talk corresponds to $n_{s c}=1,13$ and 21 ). These are the same cases in which $\varepsilon_{\text {Leak }}$ is much higher for CO-DPD than CTC-DPD. This can be seen in Fig. A.3 in Appendix A. In all the other cases CO-DPD and CTC-DPD perform almost identical. There is a direct correlation between the linearization performance loss and high values of $\varepsilon_{\text {Leak }}$ for CO-DPD. This suggests that CTC-DPD improves the linearization performance compared to CO-DPD only for low PAPR signals and relatively high PA non-linearity at strong cross-talk levels.

The error in the post-distorter estimation, $\varepsilon_{L S}$, is considerably higher for $k=1$ compared to $k=2, . .5$ because the average output power is higher for $k=1 \mathrm{com}$ pared to $k=2, \ldots 5$. This can also be seen as the predistorted input signal with a higher PAPR $(k=2 \ldots 5)$ drives the PA in to the non-linear region less frequently compared to $k=1$. The lower the non-linearity, the lower is $\varepsilon_{L S}$ and vice versa. So, $\varepsilon_{L S}$ is higher for low PAPR signals and PA responses which are highly non-linear and vice versa. $\varepsilon_{L S}$ is almost the same for CO-DPD and CTC-DPD with frequency flat cross-talk, in all the examined cases. This suggests that the cross-over structure performs as good as the cross-talk canceling structure as a post-distorter. But the 


\begin{tabular}{|c|c|c|c|c|c|c|c|c|c|c|c|c|c|c|c|c|c|c|c|c|}
\hline \multicolumn{4}{|c|}{ 己 } & \multicolumn{4}{|c|}{$\underset{E}{\stackrel{m}{*}}$} & \multicolumn{4}{|c|}{ ᄅ } & \multicolumn{4}{|c|}{ 워 } & & & & & \\
\hline \multicolumn{2}{|c|}{$c_{0}^{1}$} & \multicolumn{2}{|c|}{$\frac{1}{c r}$} & \multicolumn{2}{|c|}{ d. } & \multicolumn{2}{|c|}{$\stackrel{1}{e r}$} & \multicolumn{2}{|c|}{ d. } & \multicolumn{2}{|c|}{$\frac{1}{c r}$} & \multicolumn{2}{|c|}{$\underbrace{\prime}_{0}$} & \multicolumn{2}{|c|}{$\stackrel{1}{e r}$} & \multicolumn{4}{|c|}{ 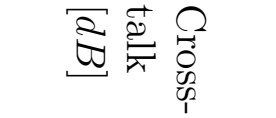 } & \\
\hline $\begin{array}{l}\text { pu } \\
1\end{array}$ & $N$ & $\begin{array}{l}0 \\
1 \\
0\end{array}$ & $\sim$ & $\begin{array}{l}\stackrel{N}{c} \\
\dot{c}\end{array}$ & $\mapsto$ & $\begin{array}{l}\text { v } \\
\dot{c}\end{array}$ & $\bullet$ & 感 & $N$ & $\begin{array}{l}0 \\
\vdots \\
b\end{array}$ & $N$ & $\begin{array}{l}c \\
1 \\
b\end{array}$ & $N$ & o & $N$ & $\begin{array}{l}\forall \\
\forall \\
\downarrow \\
\downarrow\end{array}$ & $\stackrel{5}{\leftarrow}$ & 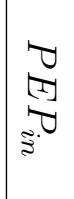 & 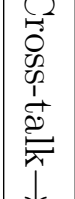 & \\
\hline$\stackrel{\infty}{\infty}$ & 光 & $\stackrel{\infty}{\stackrel{\infty}{\bullet}}$ & $\stackrel{b}{\stackrel{b}{c}}$ & 怘 & 峁 & 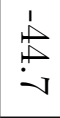 & 崩 & $\begin{array}{l}6 \\
0\end{array}$ & $\stackrel{\bullet}{\stackrel{\bullet}{\bullet}}$ & $\begin{array}{l}\text { के } \\
\text { is }\end{array}$ & $\stackrel{\oplus}{i}$ & $\stackrel{\bullet}{\oplus}$ & $\stackrel{+}{N}$ & $\stackrel{\bullet}{\triangleright}$ & $\stackrel{\bullet}{\infty}$ & $\Omega$ & $\frac{\Omega}{\sigma 0}$ & & & טقِ \\
\hline 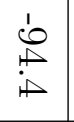 & $\begin{array}{l}1 \\
\infty \\
0 \\
0\end{array}$ & 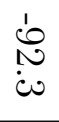 & 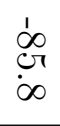 & 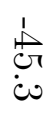 & 虫 & $\begin{array}{l}\vec{c} \\
\text { cr }\end{array}$ & 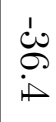 & $\stackrel{\infty}{\infty}$ & 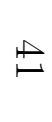 & : & $\stackrel{\bullet}{* 0}$ & $\stackrel{\bullet}{\dot{C}_{\infty}}$ & $\stackrel{\sim}{N}$ & $\stackrel{\triangleright}{\triangleright}$ & $\stackrel{F}{\varpi}$ & $\stackrel{\Omega}{\Omega}$ & $\stackrel{2}{2}$ & 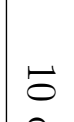 & 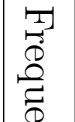 & نे \\
\hline$\stackrel{1}{\infty}$ & $\begin{array}{l}\text { dr } \\
\text { av } \\
\text { v }\end{array}$ & $\begin{array}{l}\text { ป̀ } \\
\text { v }\end{array}$ & $\stackrel{1}{\stackrel{1}{v}}$ & $\begin{array}{l}1 \\
\frac{1}{0} \\
0\end{array}$ & vِّن & $\begin{array}{l}1 \\
\stackrel{1}{0} \\
i\end{array}$ & $\underset{i \infty}{\stackrel{i}{i \infty}}$ & क्ष & $\stackrel{\bullet}{\sigma}$ & $\begin{array}{l}\text { 它 } \\
\dot{0}\end{array}$ & $\stackrel{\text { N }}{v}$ & $\stackrel{\bullet}{\sigma}$ & $\stackrel{v}{v}$ & $\stackrel{\varpi}{\sigma}$ & $\stackrel{\bullet}{N}$ & $\Omega$ & $\underset{0}{\sigma 0}$ & $\underset{B}{E}$ & 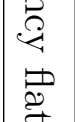 & 通 \\
\hline 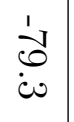 & $\frac{1}{0}$ & $\begin{array}{l}1 \\
0 \\
0 \\
\dot{c}\end{array}$ & $\begin{array}{l}1 \\
\infty \\
N \\
i\end{array}$ & $\underset{\infty}{+}$ & טِّن & 占 & مَّنْ & 宫 & $\stackrel{⿱ t}{i}$ & $\stackrel{\bullet}{i v}$ & $\begin{array}{l}\stackrel{N}{0} \\
\dot{c}\end{array}$ & $\stackrel{\bullet}{\vec{\perp}}$ & نै & $\vdash$ & $\stackrel{\bullet}{i}$ & $\stackrel{\Omega}{\Omega}$ & $\begin{array}{l}\stackrel{D}{2} \\
-1\end{array}$ & & & $\begin{array}{l}3 \\
8 \\
8 \\
\end{array}$ \\
\hline $\begin{array}{l}1 \\
\stackrel{1}{N} \\
\stackrel{1}{\vdash}\end{array}$ & $\begin{array}{l}1 \\
c \\
\dot{c} \\
\dot{c}\end{array}$ & $\stackrel{\infty}{\infty}$ & 家 & $\stackrel{1}{\stackrel{1}{c}}$ & $\begin{array}{l}\text { co } \\
\text { iv } \\
\text { iv }\end{array}$ & $\stackrel{1}{\stackrel{1}{*}}$ & $\begin{array}{l}\stackrel{1}{0} \\
i \\
i \infty\end{array}$ & $\stackrel{i v}{i v}$ & $\stackrel{+}{\infty}$ & $\stackrel{\vec{v}}{i v}$ & $\begin{array}{l}\vec{C} \\
\dot{c}\end{array}$ & $\stackrel{\bullet}{\oplus}$ & iv & $\stackrel{\bullet}{\vec{t}}$ & $\stackrel{\sim}{i}$ & 0 & $\underbrace{\infty}_{0 .}$ & & & $\begin{array}{l}\overrightarrow{0} \\
\stackrel{0}{D} \\
\vec{F}\end{array}$ \\
\hline $\begin{array}{l}1 \\
\infty \\
0 \\
\infty\end{array}$ & $\begin{array}{l}1 \\
0 \\
c \pi \\
\dot{c} 0\end{array}$ & $\begin{array}{l}1 \\
0 \\
\infty \\
\infty\end{array}$ & $\stackrel{\infty}{\infty}$ & $\begin{array}{l}\text { 离 } \\
\text { iv } \\
\text { iv }\end{array}$ & 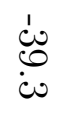 & $\begin{array}{l}1 \\
\text { cr } \\
\text { co }\end{array}$ & $\stackrel{t}{0}$ & $\stackrel{i v}{i t}$ & $\begin{array}{l}\stackrel{c}{c r} \\
i\end{array}$ & $\frac{v}{v}$ & $\begin{array}{l}\vec{c} \\
\dot{c r}\end{array}$ & $\stackrel{\bullet}{*}$ & $\stackrel{\sim}{i}$ & $\dot{\Gamma}$ & i & $\frac{\Omega}{2}$ & $\stackrel{2}{D}$ & $e^{v}$ & 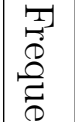 & 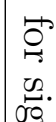 \\
\hline 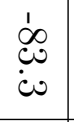 & 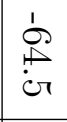 & $\begin{array}{l}1 \\
\text { do } \\
\text { do }\end{array}$ & 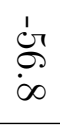 & 㑨 & 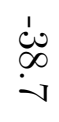 & 党 & مِ & $\begin{array}{l}\overrightarrow{0} \\
\dot{c}\end{array}$ & $\stackrel{\theta}{\circ}$ & $\begin{array}{l}6 \\
6 \\
07\end{array}$ & $\frac{v}{v}$ & $\begin{array}{l}0 \\
\dot{g} \\
\text { g }\end{array}$ & $\begin{array}{l}\circ \\
\infty \\
\infty\end{array}$ & $\begin{array}{l}\stackrel{0}{1} \\
\dot{g}\end{array}$ & $\underset{\infty}{0}$ & 3 & $\tau^{\Omega}$ & $\underset{B}{\sigma}$ & $\begin{array}{l}\overrightarrow{2} \\
\overrightarrow{4} \\
\vec{D}\end{array}$ & $\stackrel{\nu}{[-1]}$ \\
\hline$\stackrel{1}{\perp}$ & $\begin{array}{l}1 \\
\stackrel{1}{\circ}\end{array}$ & 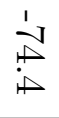 & $\frac{1}{c}$ & 怘 & $\begin{array}{l}\dot{1} \\
\infty \\
\infty \\
\infty\end{array}$ & $\begin{array}{l}1 \\
\frac{1}{c 0} \\
0\end{array}$ & 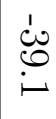 & $\begin{array}{l}\text { to } \\
\text { cr }\end{array}$ & $\begin{array}{l}6 \\
0 \\
0\end{array}$ & $\begin{array}{l}6 \\
0 \\
0\end{array}$ & $\begin{array}{l}\stackrel{+}{v} \\
\infty\end{array}$ & $\begin{array}{l}\stackrel{0}{0} \\
\stackrel{9}{g}\end{array}$ & $\begin{array}{l}\stackrel{0}{\infty} \\
\dot{\infty}\end{array}$ & $\begin{array}{l}\circ \\
\dot{8}\end{array}$ & $\stackrel{\circ}{\text { जे }}$ & $\stackrel{\Omega}{\beta}$ & $\overbrace{T}^{2}$ & & & $T_{1}$ \\
\hline 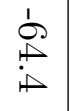 & $\begin{array}{l}1 \\
0 \\
0 \\
0\end{array}$ & & 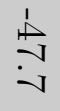 & 竞 & $\begin{array}{l}\dot{d} \\
\infty \\
0 \\
0\end{array}$ & $\begin{array}{l}\frac{1}{N} \\
\stackrel{0}{0}\end{array}$ & $\begin{array}{l}\dot{c} \\
\infty \\
\infty\end{array}$ & $\stackrel{i v}{i v}$ & $\stackrel{P}{\stackrel{P}{*}}$ & $\begin{array}{l}\vec{c} \\
\infty\end{array}$ & $\stackrel{\bullet}{\circ}$ & $\stackrel{i}{\infty}$ & •̈. & $\stackrel{\bullet}{\ddot{c} g}$ & $\dot{\sigma}$ & 0 & 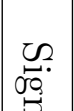 & & 3 & \\
\hline 'ّ & $\frac{1}{\partial}$ & $\begin{array}{l}1 \\
\text { a } \\
i\end{array}$ & $\begin{array}{c}\dot{b} \\
\stackrel{1}{1} \\
\infty\end{array}$ & $\stackrel{+}{\stackrel{1}{*}}$ & $\begin{array}{l}\dot{d} \\
\infty \\
\infty \\
\infty\end{array}$ & 点 & $\stackrel{1}{0}$ & $\stackrel{i v}{i v}$ & $\begin{array}{l}\vec{c} \\
\text { iv }\end{array}$ & $\stackrel{i}{i}$ & $\begin{array}{l}\text { हr } \\
\dot{c}\end{array}$ & î̀ & ט. & $\stackrel{i}{\oplus}$ & ن. & $\frac{\beta}{\beta}$ & 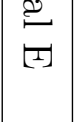 & $\begin{array}{l}\checkmark \\
2\end{array}$ & 苞 & \\
\hline $\begin{array}{l}e_{0}^{1} \\
0 \\
0\end{array}$ & $\stackrel{d}{d}$ & $\underset{0}{+1}$ & $\begin{array}{l}\dot{1} \\
\dot{c}\end{array}$ & 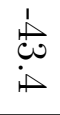 & $\begin{array}{l}\dot{1} \\
\infty \\
0 \\
0\end{array}$ & $E$ & 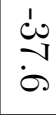 & $\begin{array}{l}60 \\
00\end{array}$ & סे & $\frac{\theta}{-v}$ & $\stackrel{t}{N}$ & $\stackrel{\circ}{-}$ & 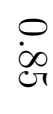 & $\stackrel{\bullet}{\ominus}$ & 官 & 2 & 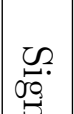 & $\vec{B}$ & $\frac{0}{0}$ & \\
\hline $\begin{array}{l}1 \\
\stackrel{1}{0} \\
\dot{c} 0\end{array}$ & $\begin{array}{l}1 \\
d \\
0 \\
\infty\end{array}$ & $\begin{array}{l}1 \\
8 \\
0\end{array}$ & $\begin{array}{l}\text { c } \\
\text { ĭ } \\
\text { i }\end{array}$ & 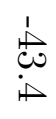 & $\begin{array}{l}c_{0} \\
\infty \\
0\end{array}$ & $\underset{i}{c}$ & $\overbrace{}^{!}$ & $\begin{array}{l}\overrightarrow{0} \\
\dot{0}\end{array}$ & $\triangleq$ & $\begin{array}{l}\overrightarrow{6} \\
i \overrightarrow{0}\end{array}$ & $\frac{1}{6}$ & $\stackrel{\circ}{-}$ & $\begin{array}{l}\stackrel{0}{\infty} \\
\stackrel{\infty}{\circ}\end{array}$ & $\stackrel{0}{v}$ & $\begin{array}{l}\stackrel{\circ}{\infty} \\
\stackrel{\theta}{ }\end{array}$ & $\frac{\Omega}{\beta}$ & $\stackrel{2}{2}$ & & $\overleftarrow{\delta}$ & \\
\hline
\end{tabular}




\section{$-15 \mathrm{~dB}$ cross-talk $\quad-20 \mathrm{~dB}$ cross-talk $\quad-30 \mathrm{~dB}$ cross-talk No cross-talk}

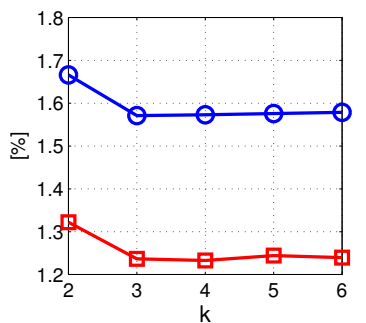

(a) EVM

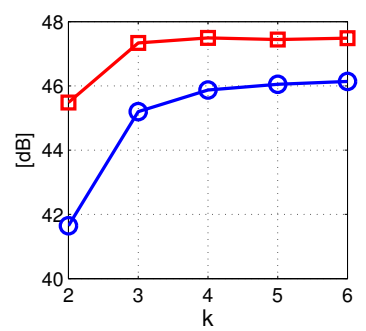

(e) ACLR

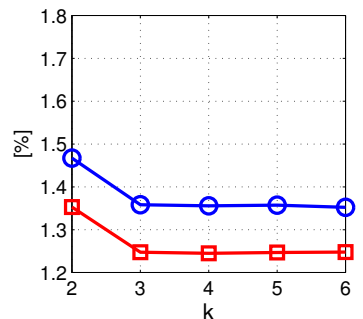

(b) EVM

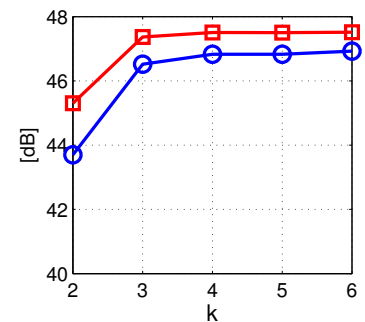

(f) ACLR

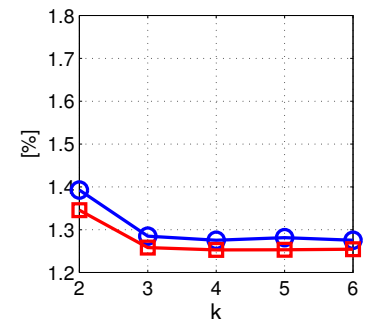

(c) EVM

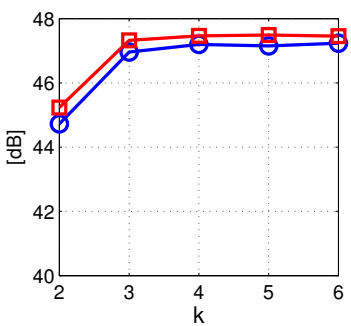

(g) ACLR

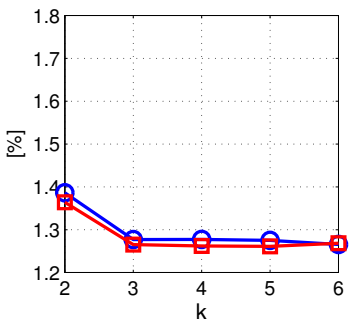

(d) EVM

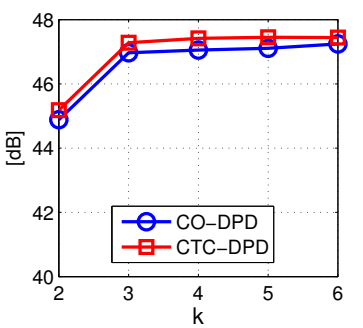

(h) ACLR

Fig. 3.18: Measurement results (EVM \& ACLR) for signal E with frequency selective cross-talk, $\mathrm{PEP}_{\text {in }}=7 \mathrm{dBm}$, using filter 1a from Fig. 3.20

differences in $\varepsilon_{\text {Leak }}$ and the linearization performance measures such as $\mathrm{NMSE}_{\mathrm{IB}}$ and EVM suggest that CO-DPD is not an accurate predistorter for MIMO transmitter. $\varepsilon_{\text {Leak }}$ for CO-DPD is higher at higher power levels for signals with lower PAPR, especially at $k=2$. This is because a higher error in DPD estimation (corresponding to $\varepsilon_{L S}$ at $k=1$ ) causes higher residual leakage for CO-DPD.

\subsubsection{Frequency selective cross-talk}

The EVM and ACLR curves for signal $\mathrm{E}$ at $\mathrm{PEP}_{\text {in }}=7 \mathrm{dBm}$ in Fig. 3.18 shows a considerable performance improvement for CTC-DPD compared to CO-DPD in the presence of strong frequency selective cross-talk. This is because the CO-DPD structure does not have a mechanism to compensate the frequency selective crosstalk, whereas the CTC-DPD structure enables the estimation of the coupling filter coefficients and thus the pre-cancellation of the cross-talk. Fig. 3.15 shows that CTCDPD performs better than CO-DPD with all the chosen transmit signals at high cross-talk levels. $\varepsilon_{L S}$ and $\varepsilon_{\text {Leak }}$ are higher for CO-DPD at higher cross-talk levels. The impulse and frequency responses of the filters used to model the cross-talk are shown in Fig. 3.19 and Fig. 3.20 respectively. In the measurements which gave the results presented in Fig. 3.15, Fig. 3.18 and Table 3.4, we used filter 1a. Fig. 3.20 shows the filter responses at $-20 \mathrm{~dB}$ cross-talk level, and other measurements use appropriately scaled versions of this filter to model the cross-talk. 


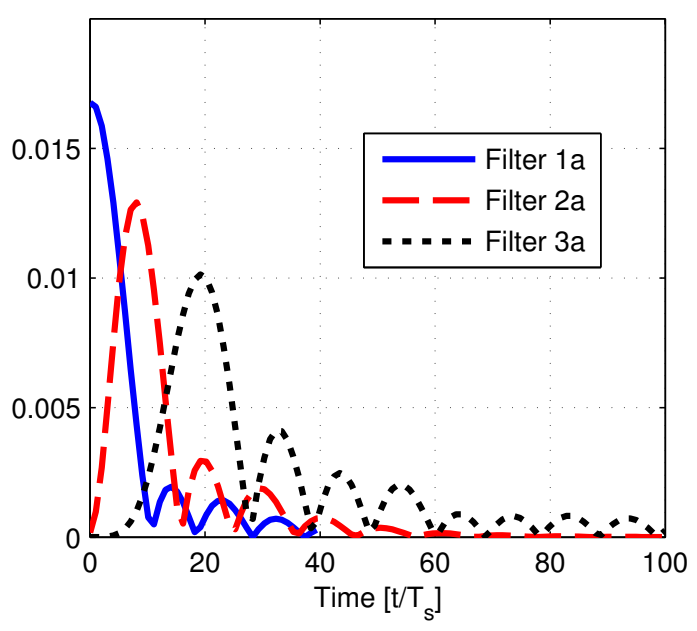

(a) Filters 1a, 2a, 3a

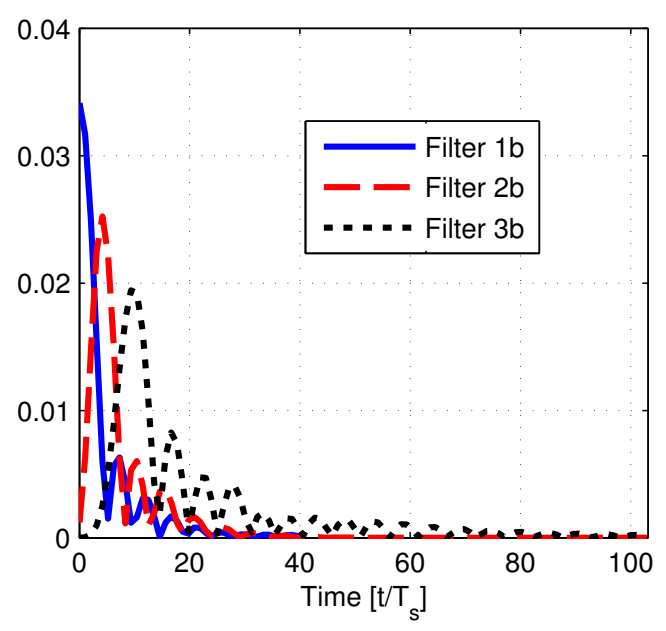

(b) Filters1b, 2b, 3b

Fig. 3.19: Impulse responses of the coupling filters, $T_{s}=1 / F_{s}$ is the sampling duration , $M=40$ for filter $1, M=100$ for filter 2,3 .

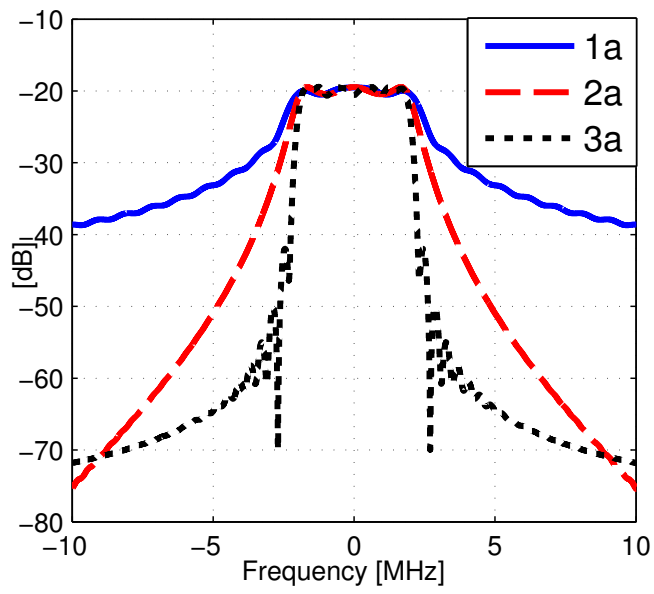

(a) Filters 1a, 2a, 3a

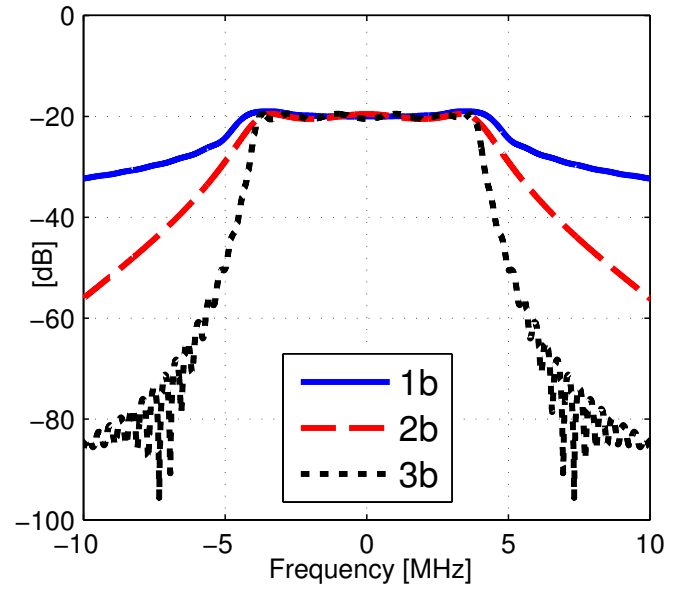

(b) Filters1b, 2b, 3b

Fig. 3.20: Frequency responses of the coupling filters applied to UMTS signals $\left(F_{s}=38.4 \mathrm{MHz}\right)$. 
Table 3.5.: Measurement results for signal E, $\mathrm{PEP}_{\text {in }}=7 \mathrm{dBm},-20 \mathrm{~dB}$, Frequency selective cross-talk

\begin{tabular}{c|c|c|c|c}
\hline \multirow{2}{*}{ Filter } & \multicolumn{2}{|c|}{ EVM [\%] } & \multicolumn{2}{c}{ ACLR [dB] } \\
\cline { 2 - 5 } & CO-DPD & CTC-DPD & CO-DPD & CTC-DPD \\
\hline \hline 1a & 1.36 & 1.25 & 46.8 & 47.5 \\
\hline 1b & 1.04 & 1.02 & 47.2 & 47.7 \\
\hline $2 \mathrm{a}$ & 2.53 & 1.23 & 44.3 & 47.2 \\
\hline 2b & 1.15 & 1.01 & 46.6 & 47.5 \\
\hline 3a & 10.04 & 1.25 & 43.8 & 47.2 \\
\hline 3b & 2.87 & 1.02 & 43.7 & 47.6 \\
\hline
\end{tabular}

We investigated the effect of the frequency selectivity of the cross-talk on the performances of CO-DPD and CTC-DPD with a UMTS signal (Signal E). We chose six low-pass filters whose impulse and frequency responses are shown in Fig. 3.19 and Fig. 3.20 respectively. The cut-off frequencies are in the order of the signal bandwidth. In reality the situation can be less severe. The filters 1a, $2 \mathrm{a}$ and $3 \mathrm{a}$ have the same cutoff frequencies as the signal, i.e, $\frac{3.84 \mathrm{MHz}}{2}=1.92 \mathrm{MHz}$ and $1 \mathrm{~b}, 2 \mathrm{~b}$ and $3 \mathrm{~b}$ have cutoff frequencies which are twice the cutoff frequency of the signal, ie. $3.84 \mathrm{MHz}$. Filters $2 \mathrm{a}$ and $2 \mathrm{~b}$ are Chebyshev filters of order 3 and $3 \mathrm{a}$ and $3 \mathrm{~b}$ are Chebyshev filters of order 6 . The pass-band ripple for all the filters is $1 \mathrm{~dB}$. All the poles of the corresponding cross-talk canceling filters $A_{i j}(z)$ for $i, j \in 1,2$, are found to be inside the unit circle, for various cross-talk levels, suggesting the possibility of a stable IIR implementation of the filters that pre-cancel the frequency selective cross-talk. The EVM and ACLR values given in Table 3.5 shows that the stronger the frequency selectivity, the larger is the improvement achieved by CTC-DPD over CO-DPD at strong cross-talk conditions.

\subsection{Measurements with cross-talk using RF components}

In the measurements presented in section 3.3 and section 3.5, the cross-talk before the PAs is applied on the baseband equivalent signals after the MIMO-DPD, in the computer. This is shown in Fig. 3.7. The resulting RF signals from the signal generator emulate the input signals to the PAs. To verify the the application of the crosstalk in software is reasonable, we carried out some measurements where the cross-talk is realized using power dividers, power combiners and attenuators as shown in Fig. 3.21. We used Huber-Suhner 4901.01A for power dividers and power combiners and Huber-Suhner 6620.19.AC and 6630.19.AC attenuators. The EVM and ACLR performance of CO-DPD and CTC-DPD for cross-talk levels -20 dB, 


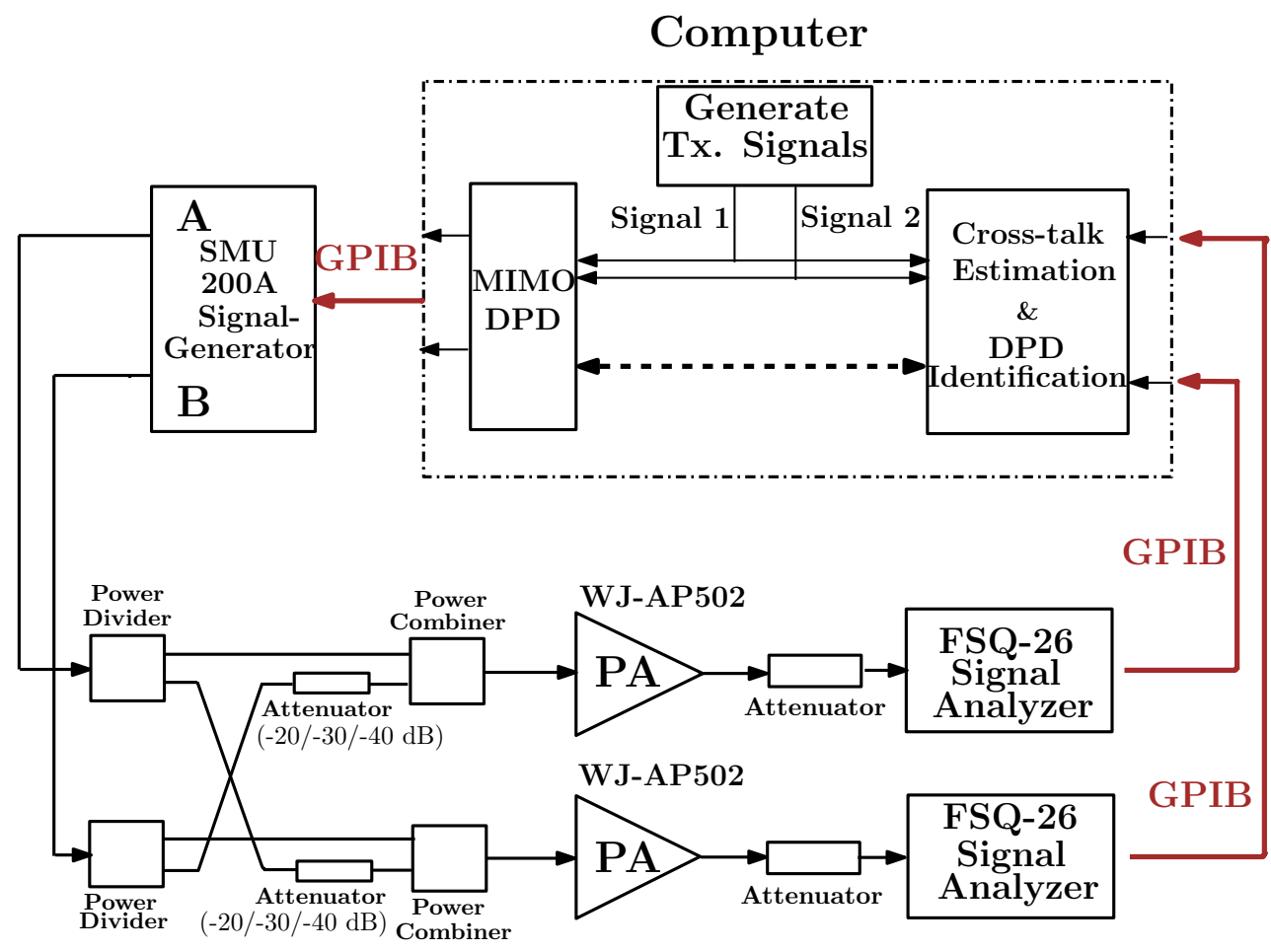

Fig. 3.21: Measurement setup for MIMO-DPDs, with cross-talk using RF components

$-30 \mathrm{~dB}$ and $-40 \mathrm{~dB}$ are shown in Fig. 3.22. In the measurement results presented in section 3.5, the peak envelope powers (PEP) of the output signals $\left(\mathrm{PEP}_{\text {out }}\right)$ were kept constant by fixing the peak envelope power of $u_{1}(n)$ and $u_{2}(n)\left(\mathrm{PEP}_{\text {in }}\right)$. Here, this is not possible. So we fix the PEP of $x_{1}(n)$ and $x_{2}(n)$ to be $7 \mathrm{dBm}$. Because of this, there is a slight difference in EVM and ACLR performance compared to what is presented in Fig. 3.16. The solid graphs represent the measurements with cross-talk generated using RF components. The dotted lines represent the measurements with cross-talk simulated in computer, which is presented in Fig. 3.16. These measurement results show that CO-DPD and CTC-DPD perform as expected, in the presence of cross-talk generated using RF components. This means that the measurement results with the computer generated cross-talk are reliable. 


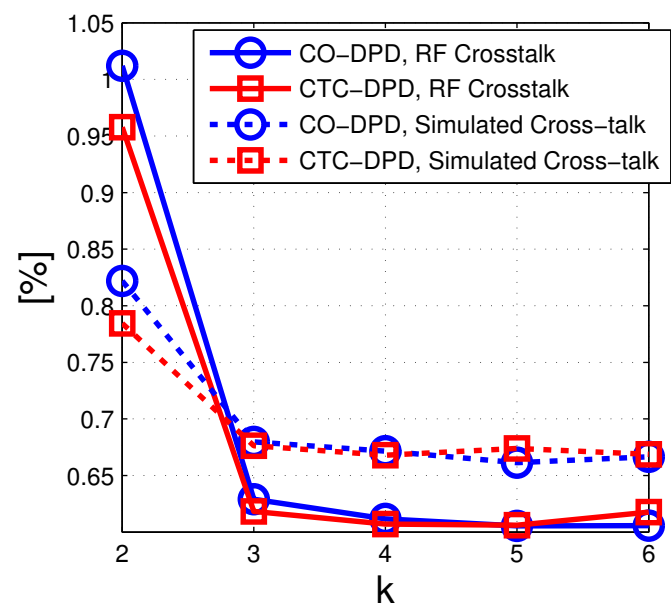

(a) EVM, $-20 \mathrm{~dB}$ cross-talk

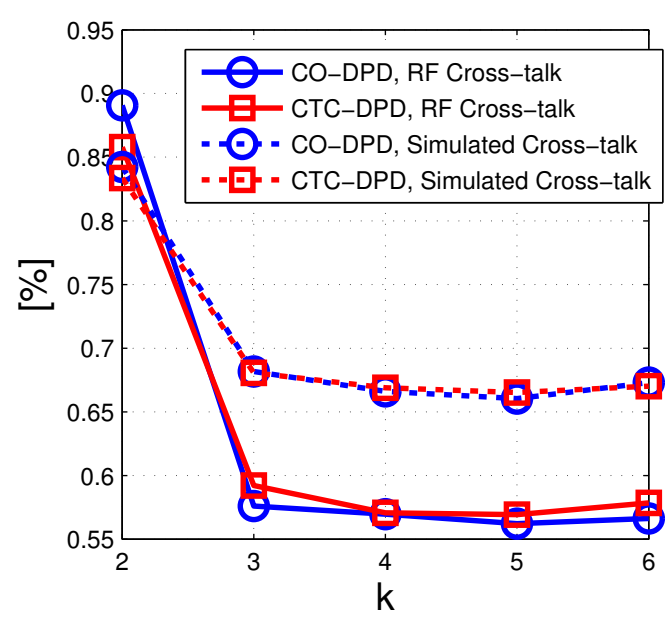

(c) EVM, -30 dB cross-talk

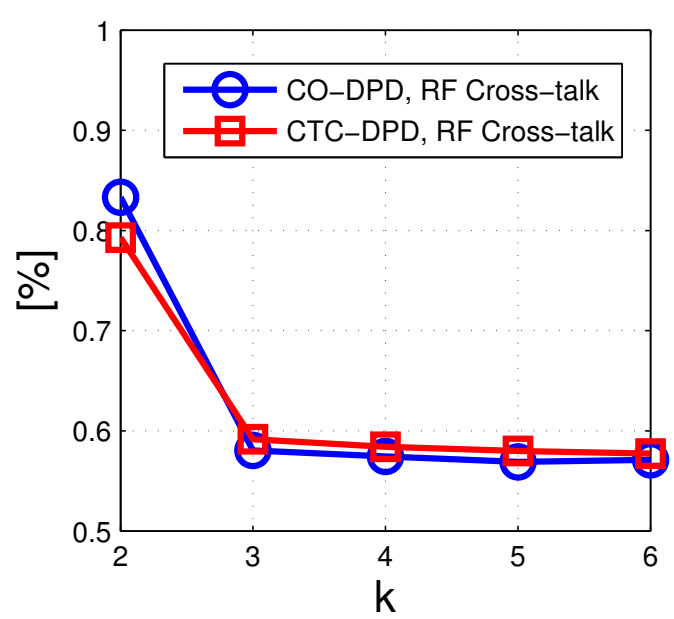

(e) EVM, -40 dB cross-talk

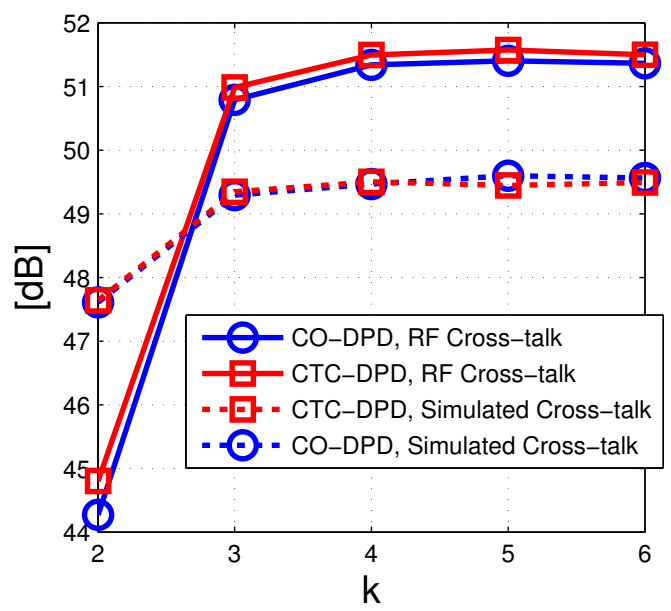

(b) ACLR, $-20 \mathrm{~dB}$ cross-talk

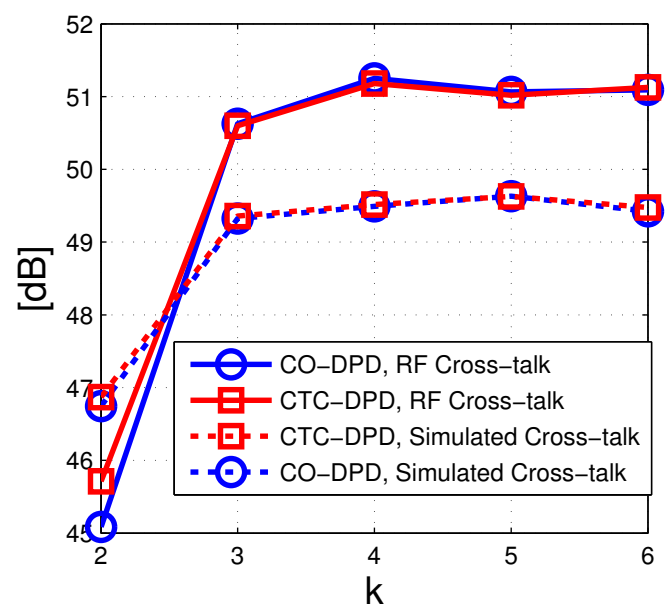

(d) ACLR, $-30 \mathrm{~dB}$ cross-talk

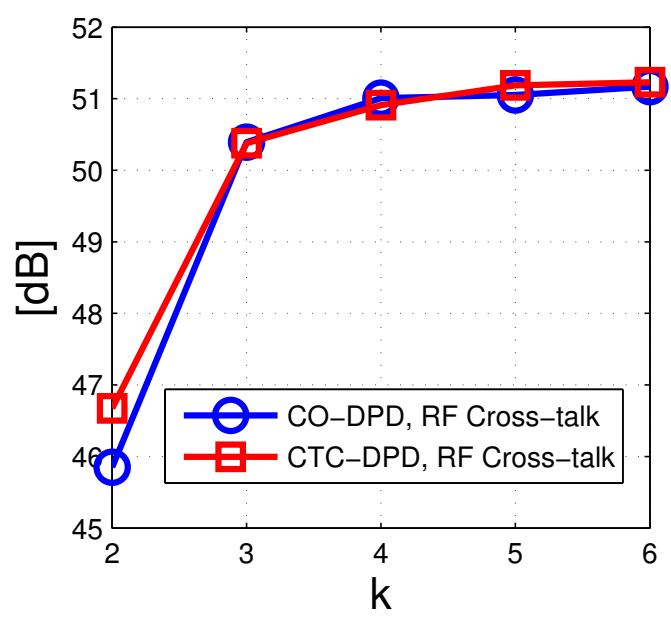

(f) ACLR, $-40 \mathrm{~dB}$ cross-talk

Fig. 3.22: Measurement results (EVM \& ACLR) for signal F, with frequency flat cross-talk using RF components, PEP of $x_{1}(n)$ and $x_{2}(n)=7 \mathrm{dBm}$ 


\section{Chapter 4.}

\section{Adaptive predistortion for MIMO transmitters}

In this chapter, we discuss the adaptive algorithms for CO-DPD and CTC-DPD for multiple antenna transmitters. Ideally, adaptive predistortion requires an update of the DPD coefficients every sample duration. This can lead to prohibitively complex hardware requirements in terms of speed and complexity. Moreover, to adaptively adjust the predistorter function, a receiver must down-convert the PA output signal, for the computation of the DPD coefficients [61]. In a half-duplex communication system, the already present receiver can be utilized for this purpose. In full-duplex systems, a dedicated feed-back receiver is required for this purpose. In addition, the timing of the received signal must be synchronized with a delayed version of the transmitted signal. Large delays in this transmit-receive loop can affect the convergence of the adaptive predistortion. However, adaptive DPD algorithms can be modified/simplified to circumvent the problems of complexity and loop-delays and to make them feasible for the implementation. An exmaple of such modification is the block adaptive predistortion proposed in [62].

The recursive least squares (RLS) and stochastic gradient (SG) adaptive algorithms for CO-DPD are discussed in [63]. A MIMO-PD system that linearizes the power amplifier response and compensates nonlinear crosstalk and IQ imbalance effects for each branch of the multiantenna system is proposed in this paper. We proposed the use of the RLS adaptive algorithm for CTC-DPD and gave preliminary simulation results in [12]. In this chapter, we discuss the least mean squared (LMS ${ }^{1}$ ) and RLS adaptive algorithms applied to CO-DPD and CTC-DPD. While there are advanced versions of LMS and RLS applied to DPD [64-68], our focus here is to present the basic version of these algorithms applied to CO-DPD and CTC-DPD in MIMO transmitters. So, we use the well-known standard versions [69] of LMS and RLS. We have presented a detailed discussion of the linearization performances and the computational complexities of LMS and RLS algorithms for CO-DPD and CTC$\mathrm{DPD}$ in [13]. The linearization performances of adaptive algorithms for MIMO DPDs (ie., CO-DPD and CTC-DPD) for a two-antenna transmitter are evaluated

\footnotetext{
${ }^{1}$ The LMS algorithm is an improtant member of the family of stochastic gradient algorithms.
} 
through simulations with the same set of transmit signals (QPSK, 16-QAM, UMTS and LTE) used for the measurements described in chapter 3. It is found that the performance of CO-DPD and CTC-DPD are almost identical. In the presence of frequency selective cross-talk, the extension of CTC-DPD which can pre-cancel such cross-talk, performs considerably better than CO-DPD, which can not take the frequency selectivity of the cross-talk in to account. A detailed discussion on the simulation results is presented in section 4.3. While the adaptive algorithms for CODPD and CTC-DPD can be extended to any number of transmit paths, we consider only two and four antenna transmitter cases for simplicity.

\subsection{Adaptive predistortion}

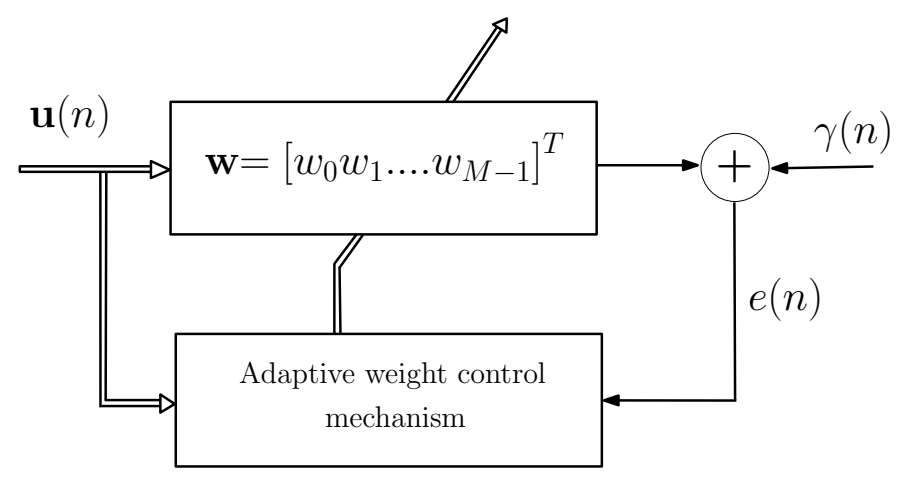

Fig. 4.1: Adaptive transversal filter

Fig. 4.1 shows an adaptive transversal filter for the linear optimum filtering problem. $\boldsymbol{u}(n)=[u(n) u(n-1) \ldots u(n-M+1)]^{T}$ denotes the vector of input samples, $\gamma(n)$ is the desired output signal and $e(n)$ is the error between the output and the estimated output signals. The filter coefficient vector $\boldsymbol{w}$ is updated every sample duration, in order to minimize some cost function $J(n)$, as the time progresses. In the steepest-descent algorithm, the cost function is chosen as the expectation of the square of the error signal, i.e, $J(n)=\mathbb{E}\left[|e(n)|^{2}\right]$. This requires the prior knowledge of the correlation matrix of $\boldsymbol{u}(n)$ and the cross-correlation vector between $\boldsymbol{u}(n)$ and $\gamma(n)$. The simplest choice for estimates of these quantities, which in general are not known, are their instantaneous values. This choice leads to the least mean squares (LMS) adaptive filter [69,70]. It immediately follows that the correlation matrix of $\boldsymbol{u}(n)$ is approximated as $\boldsymbol{u}(n) \boldsymbol{u}^{H}(n)$ and the cross-correlation vector between $\boldsymbol{u}(n)$ and $\gamma(n)$ as $\boldsymbol{u}(n) \gamma^{*}(n)$. The LMS algorithm can be described as

$$
\begin{gathered}
\boldsymbol{w}_{L M S}(0)=\boldsymbol{w}_{\text {init }} \\
e(n)=\gamma_{L M S}(n)-\boldsymbol{w}_{L M S}^{H}(n-1) \boldsymbol{u}_{L M S}(n) \\
\boldsymbol{w}_{L M S}(n)=\boldsymbol{w}_{L M S}(n-1)+\mu \boldsymbol{u}_{L M S}(n) e^{*}(n)
\end{gathered}
$$


where $\boldsymbol{w}_{L M S}(n)$ is the vector of the LMS filter coefficients, $\boldsymbol{u}_{L M S}(n)$ is the input vector and $\gamma_{L M S}(n)$ is the desired output signal at the $n^{\text {th }}$ sample duration. $\boldsymbol{w}_{\text {init }}$ is the initialization vector and $\mu$ is the step-size parameter in LMS algorithm. The filter coefficients are updated every sample according to (4.1c).

In the recursive least square (RLS) method $[69,71]$, the cost function is

$$
J(n)=\sum_{i=1}^{n} \lambda^{n-i}|e(i)|^{2}+\delta \lambda^{n}\|w(n)\|^{2}
$$

The RLS algorithm can be described as

$$
\begin{gathered}
\boldsymbol{w}_{R L S}(0)=\boldsymbol{w}_{\text {init }}, \quad \boldsymbol{P}(0)=\delta^{-1} \boldsymbol{I} \\
\boldsymbol{\pi}(n)=\boldsymbol{P}(n-1) \boldsymbol{u}_{R L S}(n) \\
\boldsymbol{k}(n)=\frac{\boldsymbol{\pi}(n)}{\lambda+\boldsymbol{u}_{R L S}^{H}(n) \boldsymbol{\pi}(n)} \\
e(n)=\gamma_{R L S}(n)-\boldsymbol{w}_{R L S}^{H}(n-1) \boldsymbol{u}_{R L S}(n) \\
\boldsymbol{w}_{R L S}(n)=\boldsymbol{w}_{R L S}(n-1)+\boldsymbol{k}(n) e^{*}(n) \\
\boldsymbol{P}(n)=\lambda^{-1} \boldsymbol{P}(n-1)-\lambda^{-1} \boldsymbol{k}(n) \boldsymbol{u}_{R L S}^{H}(n) \boldsymbol{P}(n-1)
\end{gathered}
$$

where $\boldsymbol{w}_{R L S}(n)$ is the vector of the RLS filter coefficients, $\boldsymbol{u}_{R L S}(n)$ is the input vector and $\gamma_{R L S}(n)$ is the desired output signal at the $n^{\text {th }}$ sample duration. $\boldsymbol{w}_{\text {init }}$ is the initialization vector, $\boldsymbol{I}$ is the identity matrix, $\lambda$ is the forgetting factor and $\delta$ is the regularization parameter in the RLS algorithm. $\boldsymbol{P}(n)$ is the inverse correlation matrix and $\boldsymbol{k}(n)$ is the gain vector. The filter coefficients are updated every sample according to (4.3e).

The LMS and RLS filters can be used for the adaptive predistortion as described later in this section, and for the estimation of coupling factors for CTC-DPD as described in section 4.2. The post-distorter output of a single antenna transmitter is

$$
\hat{x}(n)=\sum_{q=0}^{Q} \sum_{\substack{k=1 \\ k \text { odd }}}^{K} d_{k, q}|y(n-q)|^{k-1} y(n-q),
$$

where $y(n)$ is the complex envelope of the RF signal at the PA output attenuated by the small signal gain of the PA. $K$ and $Q$ are the maximum polynomial order and memory depth, respectively. $d_{k, q}$ is the $k^{\text {th }}$ polynomial coefficient of the $q^{\text {th }}$ filter tap. (4.4) can be written as

$$
\hat{x}(n)=\boldsymbol{d}^{H} \boldsymbol{y}(n),
$$

where $\boldsymbol{d}=\left[\begin{array}{lllllll}d_{1,0} & d_{1,1} & \ldots & d_{1, Q} & d_{3,0} \ldots d_{K, 0} & \ldots & d_{K, Q}\end{array}\right]^{H}$ is the $L \times 1$ DPD coefficient vector, where $L=Q(K+1) / 2 . \boldsymbol{y}(n)=\left[a_{1}(y(n)) a_{1}(y(n-1)) \ldots a_{1}(y(n-Q)) \ldots\right.$ $\left.a_{3}(y(n)) \ldots a_{K}(y(n)) \ldots a_{K}(y(n-Q))\right]^{T}$ and $a_{k}(y)=|y|^{k-1} \cdot y$. The DPD coeffi- 
cient vector $\boldsymbol{d}(n)$ can be updated every sample by the LMS or RLS algorithm.

$$
\begin{aligned}
& \boldsymbol{w}_{\text {init }}=\left[\begin{array}{lll}
1 & 0 & 0 \ldots 0
\end{array}\right]^{T} \\
& \boldsymbol{w}_{L M S / R L S}(n)=\boldsymbol{d}(n) \\
& \boldsymbol{u}_{L M S / R L S}(n)=\boldsymbol{y}(n) \\
& \gamma_{L M S / R L S}(n)=x(n)
\end{aligned}
$$

The adaptive algorithms for CO-DPD and CTC-DPD are discussed in section 4.2.

\subsection{Adaptive algorithms for cross-over and cross-talk canceling DPDs}

We apply the LMS and RLS adaptive algorithms to CO-DPD and CTC-DPD structures which are discussed in section 3.2. The post-distorter outputs in the CO-DPD structure for a two antenna transmitter are

$$
\hat{x}_{1}(n)=\boldsymbol{D}_{1}^{H} \boldsymbol{\psi}(n), \hat{x}_{2}(n)=\boldsymbol{D}_{2}^{H} \boldsymbol{\psi}(n),
$$

where $\boldsymbol{\psi}(n)=\left[\begin{array}{lll}\boldsymbol{y}_{1}^{T}(n) & \boldsymbol{y}_{2}^{T}(n)\end{array}\right]^{T}$ is the output signal vector, $\boldsymbol{D}_{1}=\left[\begin{array}{ll}\boldsymbol{d}_{11}^{T} & \boldsymbol{d}_{12}^{T}\end{array}\right]^{T}$ and $\boldsymbol{D}_{2}=\left[\begin{array}{ll}\boldsymbol{d}_{21}^{T} & \boldsymbol{d}_{22}^{T}\end{array}\right]^{T}$ are the CO-DPD coefficients. $\boldsymbol{y}_{i}(n)=\left[\begin{array}{ll}a_{1}\left(y_{i}(n)\right) & a_{1}\left(y_{i}(n-1)\right)\end{array}\right.$ $\left.\ldots a_{1}\left(y_{i}(n-Q)\right) a_{3}\left(y_{i}(n)\right) \ldots a_{K}\left(y_{i}(n)\right) \ldots a_{K}\left(y_{i}(n-Q)\right)\right]^{T}$ for $i=1,2$. The LMS/RLS adaptive algorithm for $\boldsymbol{D}_{1}$ in CO-DPD is

$$
\begin{aligned}
& \boldsymbol{w}_{\text {init }}=\left[\begin{array}{lll}
1 & 0 & 0 \ldots . .0
\end{array}\right]^{T} \\
& \boldsymbol{w}_{L M S / R L S}(n)=\boldsymbol{D}_{1}(n) \\
& \boldsymbol{u}_{L M S / R L S}(n)=\boldsymbol{\psi}(n) \\
& \gamma_{L M S / R L S}(n)=x_{1}(n)
\end{aligned}
$$

$\boldsymbol{D}_{2}$ can be estimated in a similar way. In the case of CTC-DPD, the two predistorters $\boldsymbol{d}_{1}$ and $\boldsymbol{d}_{2}$ are separately estimated using the LMS/RLS algorithms. The accuracy of predistortion depends on the quality of cross-talk cancellation. The cross-talk coefficients can be estimated from the samples of input signals to the MIMO transmitter $\left(x_{1}\right.$ and $\left.x_{2}\right)$ and post-distorter output $\left(\hat{v}_{1}\right.$ and $\left.\hat{v}_{2}\right)$ as shown in Fig. 3.4. These signals are related $\mathrm{as}^{2}$

$$
\begin{aligned}
& \hat{v}_{1}(n)=x_{1}(n)+\boldsymbol{\alpha}_{1}^{T} \boldsymbol{x}_{2}(n)+n_{1}(n) \\
& \hat{v}_{2}(n)=x_{2}(n)+\boldsymbol{\alpha}_{2}^{T} \boldsymbol{x}_{1}(n)+n_{2}(n)
\end{aligned}
$$

where $\boldsymbol{x}_{i}=\left[\begin{array}{llll}x_{i}(n) & x_{i}(n-1) & \ldots . x_{i}(n-M+1)\end{array}\right]^{T}$ and $\boldsymbol{\alpha}_{i}=\left[\begin{array}{lll}\alpha_{i, 0} & \alpha_{i, 1} & \ldots . \alpha_{i, M-1}\end{array}\right]^{T}$ for $i=1,2$ and $n_{1}$ and $n_{2}$ are noise samples modeling the measurement noise and

\footnotetext{
${ }^{2}$ Unlike the cross-talk estimation for block-based CTC-DPD described in chapter 3, we don't need to select the linear section of the PA response here.
} 
the non-perfect linearization. In the case of frequency flat cross-talk $(M=1)$, $\boldsymbol{\alpha}_{i}$ and $\boldsymbol{x}_{i}$ are scalars. We proposed the LMS, RLS and low-pass filtering (LPF) methods for adaptively estimating the cross-talk coefficients in [12]. The estimates of the coupling coefficients - which are used in the pre-cancellation of cross-talk in CTC-DPD - are updated every sample duration using these methods. Adaptive cross-talk estimation run along with the adaptive predistortion algorithms for the two transmitters, but it is not shown in Fig. 3.4 for simplicity. Now, we discuss these adaptive cross-talk estimation methods in detail.

The RLS/LMS estimation for $\boldsymbol{\alpha}_{1}$ is

$$
\begin{aligned}
& \boldsymbol{w}_{\text {init }}=\left[\begin{array}{lll}
0 & 0 & 0 \ldots 0
\end{array}\right]^{T} \\
& \boldsymbol{w}_{L M S / R L S}(n)=\hat{\boldsymbol{\alpha}}_{1}^{*}(n) \\
& \boldsymbol{u}_{L M S / R L S}(n)=\boldsymbol{x}_{2}(n) \\
& \gamma_{L M S / R L S}(n)=\hat{v}_{1}(n)-x_{1}(n)
\end{aligned}
$$

An estimate of $\boldsymbol{\alpha}_{2}$ can be obtained in a similar way. A block of $M$ samples of $\hat{v}_{1}(n)$ and $\hat{v}_{2}(n)$ can be written as

$$
\begin{aligned}
& \hat{\boldsymbol{v}}_{1}(n)=\boldsymbol{x}_{1}(n)+\boldsymbol{X}_{2}(n) \boldsymbol{\alpha}_{1}+\boldsymbol{n}_{\mathbf{1}}(n) \\
& \hat{\boldsymbol{v}}_{2}(n)=\boldsymbol{x}_{2}(n)+\boldsymbol{X}_{1}(n) \boldsymbol{\alpha}_{2}+\boldsymbol{n}_{2}(n)
\end{aligned}
$$

where $\hat{\boldsymbol{v}}_{i}=\left[\begin{array}{llll}\hat{v}_{i}(n) & \hat{v}_{i}(n-1) \ldots \hat{v}_{i}(n-M+1)\end{array}\right]^{T}$ and

$$
\boldsymbol{X}_{i}(n)=\left[\begin{array}{cccc}
x_{i}(n) & x_{i}(n-1) & . . & x_{i}(n-M+1) \\
x_{i}(n-1) & x_{i}(n-2) & . . & x_{i}(n-M) \\
. . & . . & . . & . . \\
x_{i}(n-M+1) & x_{i}(n-M) & . . & x_{i}(N-2 M+1)
\end{array}\right]
$$

for $i=1,2$. From (4.11), it can be seen that $\boldsymbol{X}_{2}^{-1}(n)\left(\hat{v}_{1}(n)-x_{1}(n)\right)$ gives a sample by sample estimate of $\alpha_{1}$. A low pass-filter (LPF) can be applied to this estimate to reduce the effect of noise. The LPF method for cross-talk estimation is

$$
\begin{gathered}
\hat{\boldsymbol{\alpha}}_{1}(0)=0 \\
\hat{\boldsymbol{\alpha}}_{1}(n)=r \cdot \hat{\boldsymbol{\alpha}}_{1}(n-1)+ \\
(1-r) \cdot\left(\boldsymbol{X}_{2}(n)+\delta^{\prime} \boldsymbol{I}\right)^{-1}\left(\hat{v}_{1}(n)-x_{1}(n)\right)
\end{gathered}
$$

where $\delta^{\prime}$ is a regularization parameter.

The CO-DPD and CTC-DPD structures for a four antenna transmitter are described in section 3.2. The LMS and RLS adaptive algorithms can be easily applied to this case. For a four antenna transmitter, the cross-talk before the PAs is described in (3.16). Similar to (4.8), the LMS/RLS adaptive algorithm for CO-DPD can be 


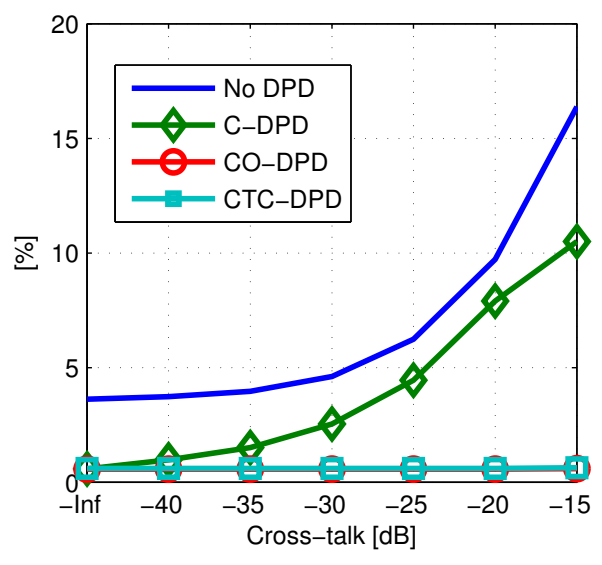

(a) EVM

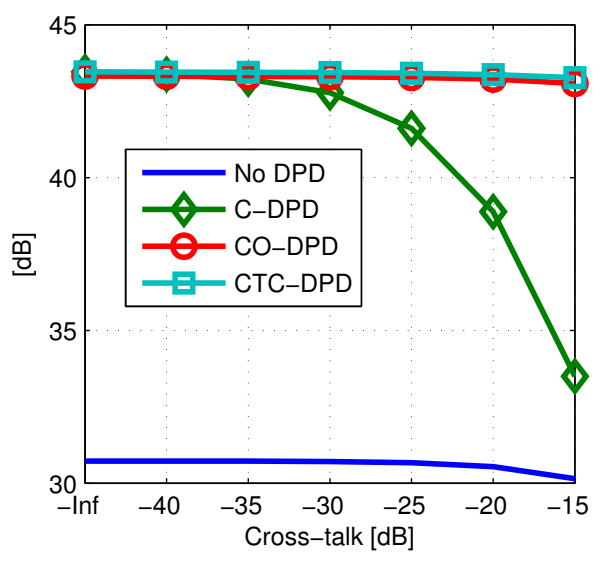

(b) ACLR

Fig. 4.2: Comparison of C-DPD and MIMO-DPDs, LMS-DPD, $N_{t}=2$, signal B, $\mathrm{PA}_{1}$, Frequency flat cross-talk.

expressed as

$$
\begin{aligned}
& \boldsymbol{w}_{\text {init }}=\left[\begin{array}{lll}
1 & 0 & 0 \ldots .
\end{array}\right]^{T} \\
& \boldsymbol{w}_{L M S / R L S}(n)=\boldsymbol{D}_{i}(n) \\
& \boldsymbol{u}_{L M S / R L S}(n)=\boldsymbol{\psi}(n) \\
& \gamma_{L M S / R L S}(n)=x_{i}(n)
\end{aligned}
$$

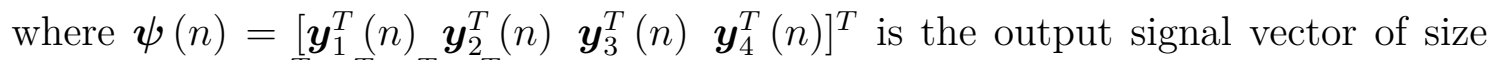

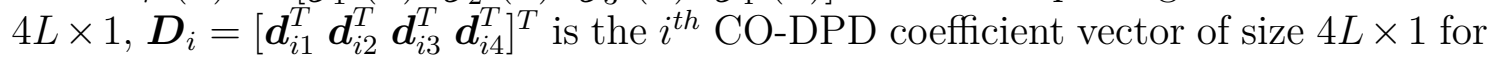
$i=1,2,3,4$. For the operation of CTC-DPD, the LMS/RLS cross-talk estimation methods described in (4.10) can be extended as

$$
\begin{aligned}
& \boldsymbol{w}_{\text {init }}=\left[\begin{array}{lll}
0 & 0 & 0
\end{array}\right]^{T} \\
& \boldsymbol{w}_{L M S / R L S}(n)=\hat{\boldsymbol{\beta}}_{i}^{*}(n) \\
& \boldsymbol{u}_{L M S / R L S}(n)=\boldsymbol{x}_{i}^{c}(n) \\
& \gamma_{L M S / R L S}(n)=\hat{v}_{i}(n)-x_{i}(n)
\end{aligned}
$$

for $i \in\{1,2,3,4\} . \hat{\boldsymbol{\beta}}_{i}=\left[\hat{\beta}_{i r} \hat{\beta}_{i s} \hat{\beta}_{i t}\right]^{T}$ is the estimate of crosstalk coefficient vector and $\boldsymbol{x}_{i}^{c}(n)=\left[x_{r}(n) x_{s}(n) x_{t}(n)\right]^{T}$, where $r, s, t \in\{1,2,3,4\}$ and $r, s, t \neq i$. $\beta_{i j}$ is the coupling factor which model the cross-talk from the $j^{\text {th }}$ transmit path to the $i^{\text {th }}$ transmit path.

\subsection{Simulation results and discussion}

The performance of C-DPD and the MIMO-DPDs are evaluated through simulations. The PA responses used in the simulations are estimated from the input 


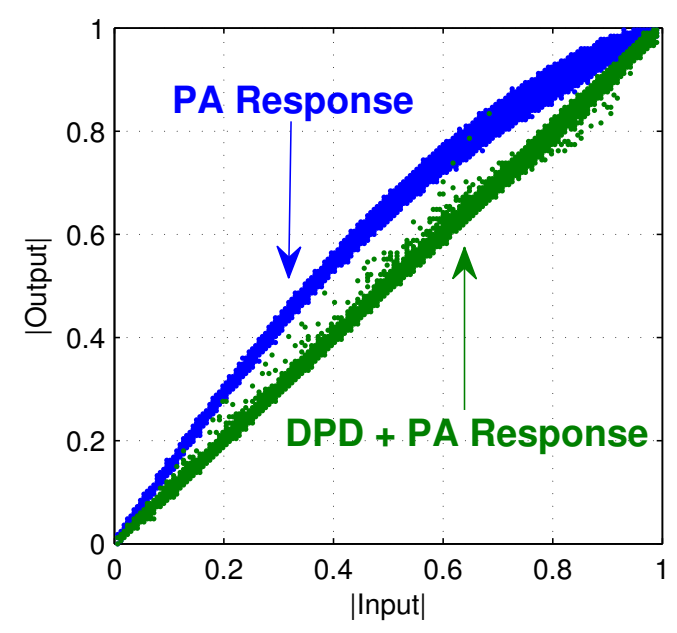

(a) $\mathrm{PA}_{1}$

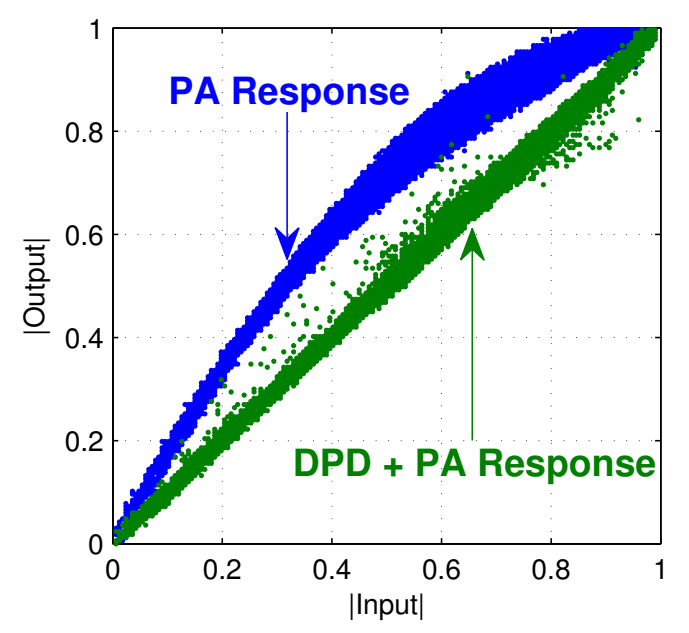

(b) $\mathrm{PA}_{2}$

Fig. 4.3: AM-AM plots (LMS-DPD), Signal D.

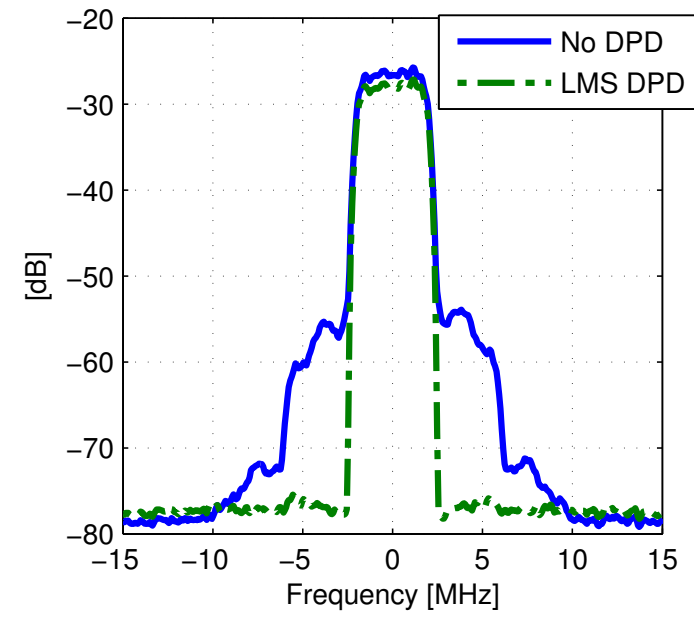

(a) $\mathrm{PA}_{1}$

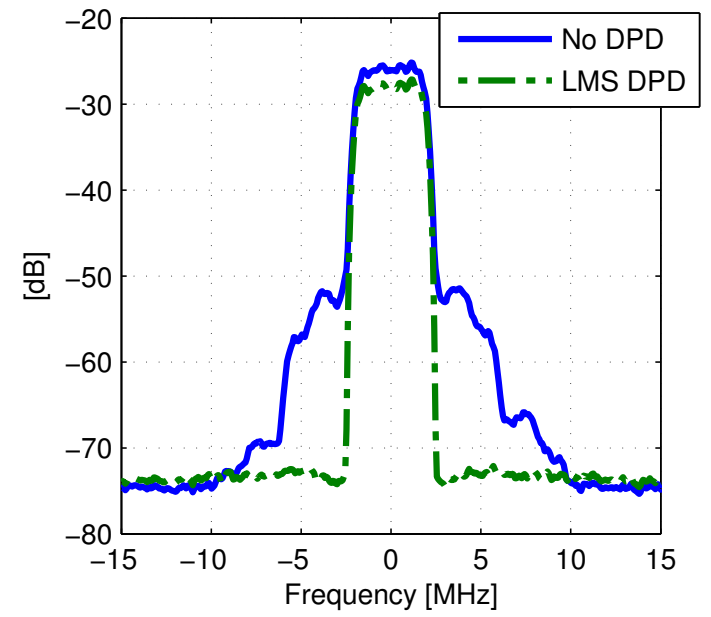

(b) $\mathrm{PA}_{2}$

Fig. 4.4: Spectra (LMS-DPD), Signal D. 
Table 4.1.: Details of the simulation setup

\begin{tabular}{|c|c|c|c|}
\hline \multirow{2}{*}{\multicolumn{2}{|c|}{$\begin{array}{l}\text { Memory polynomial } \\
\text { model }\end{array}$}} & $K$ & 9 \\
\hline & & $Q$ & 3 \\
\hline \multicolumn{3}{|c|}{ Power amplifier (PA) } & WJ-AP502 \\
\hline \multicolumn{3}{|c|}{ Supply voltage to the PA } & $7 \mathrm{~V}$ \\
\hline \multicolumn{3}{|c|}{ Bias voltage to the PA } & $3.6 \mathrm{~V}$ \\
\hline \multicolumn{3}{|c|}{ Center frequency } & $2140 \mathrm{MHz}$ \\
\hline \multicolumn{3}{|c|}{ Signal to modeling error ratio } & $38 \mathrm{~dB}$ to $45 \mathrm{~dB}$ \\
\hline \multicolumn{3}{|c|}{ Signal to measurement noise ratio } & $80 \mathrm{~dB}$ to $90 \mathrm{~dB}$ \\
\hline \multirow{2}{*}{\multicolumn{2}{|c|}{$\begin{array}{l}\text { Adaptive } \\
\text { DPD }\end{array}$}} & RLS & $\lambda=0.99, \quad \delta=0.1$ \\
\hline & & LMS & $\mu=0.1$ \\
\hline \multirow{6}{*}{$\begin{array}{c}\text { Estimation of } \\
\text { Coupling Coefficients } \\
\text { (CTC-DPD) }\end{array}$} & \multirow{3}{*}{$\begin{array}{l}\text { Frequecy } \\
\quad \text { flat }\end{array}$} & $\mathrm{LPF}$ & $r=0.98$ \\
\hline & & LMS & $\mu=0.1$ \\
\hline & & RLS & $\lambda=0.99, \quad \delta=0.1$ \\
\hline & \multirow{3}{*}{$\begin{array}{l}\text { Frequecy } \\
\text { selective }\end{array}$} & $\mathrm{LPF}$ & $r=0.98$ \\
\hline & & LMS & $\mu=0.01$ \\
\hline & & RLS & $\lambda=0.999, \quad \delta=0.1$ \\
\hline
\end{tabular}

and output signal samples obtained from measurements which are explained in chapter 3. The two PA responses $\mathrm{PA}_{1}$ and $\mathrm{PA}_{2}$ correspond to the measurements at $\mathrm{PEP}_{\text {in }}=7 \mathrm{dBm}\left(\mathrm{PEP}_{\text {out }}=34 \mathrm{dBm}\right)$ and $\mathrm{PEP}_{\text {in }}=10 \mathrm{dBm}\left(\mathrm{PEP}_{\text {out }}=35 \mathrm{dBm}\right)$ respectively, where $\mathrm{PEP}_{\text {in }}$ and $\mathrm{PEP}_{\text {out }}$ are the peak envelope powers of the input and output signals of the PA. Fig. 4.3 and Fig. 4.4 show the AM-AM and spectra plots. The PA modeling error is found to be dependent on the input signal magnitude. So, we use additive Gaussian noise whose variance is dependent on the input signal magnitude, to represent this dependence of the modeling error. This Gaussian noise is added to the simulated PA output signal. Measurement noise is modeled as AWGN. The range of values of signal to modeling error ratio and signal to measurement noise ratio for different transmit signals and the two PA responses are given in Table 4.1. The transmit signals, A-G considered here, are the same (QPSK, 16-QAM, UMTS and LTE signals) as discussed in chapter 3. Further details of the simulation setup are given in Table 4.1. The particular choice of $\lambda, \delta$ and $\mu$ for the RLS/LMS adaptive DPD algorithms is made to ensure acceptable levels of convergence time and stability. The values of $\lambda, \delta, \mu$ and $r$ for adaptive cross-talk estimation are also selected based on similar criteria.

The linearization performances of C-DPD, CO-DPD and CTC-DPD in a two-antenna transmitter are compared based on Error Vector Magnitude (EVM), In-band Nor- 


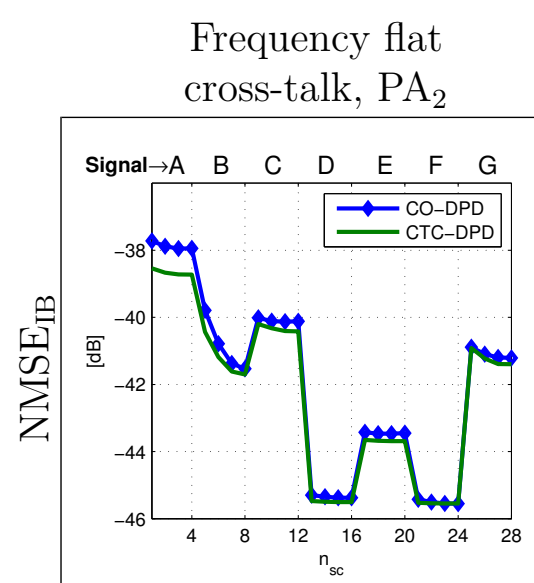

(a)

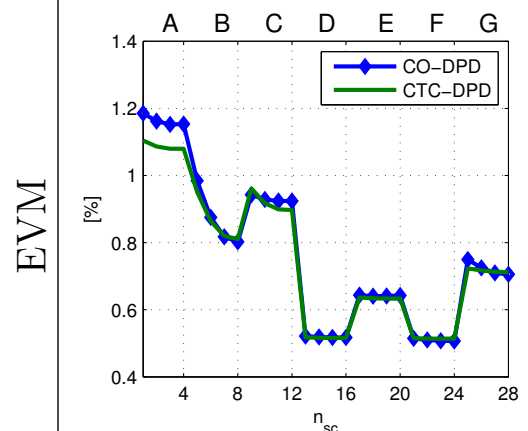

(d)
Frequency flat cross-talk, $\mathrm{PA}_{1}$
Frequency selective cross-talk, $\mathrm{PA}_{1}$

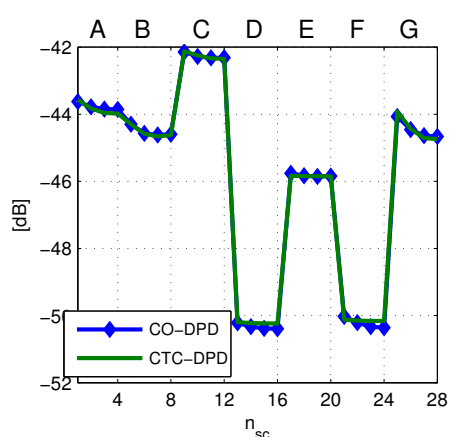

(b)

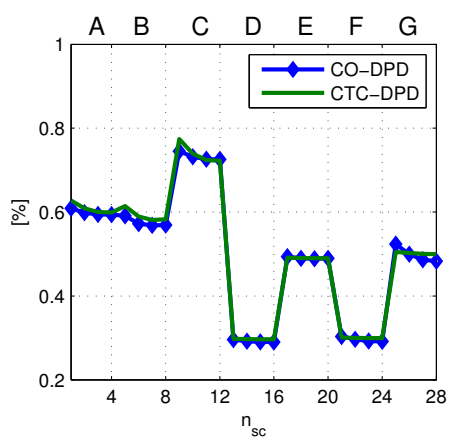

(e)

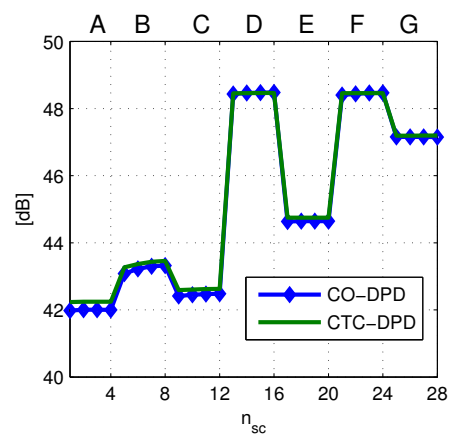

(h)

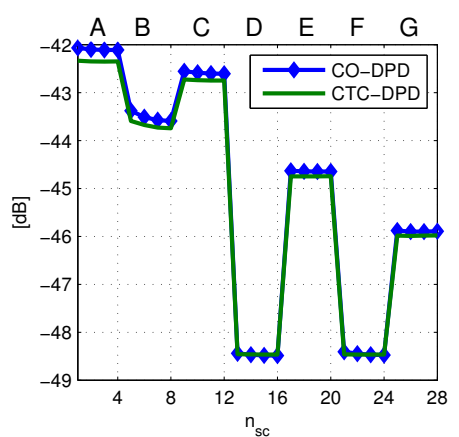

(k)

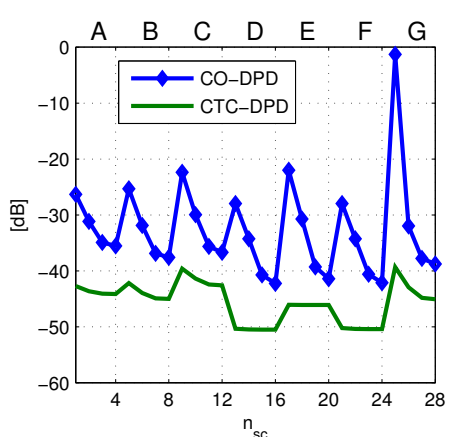

(c)

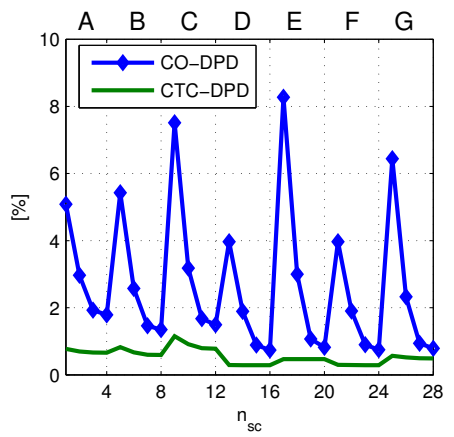

(f)

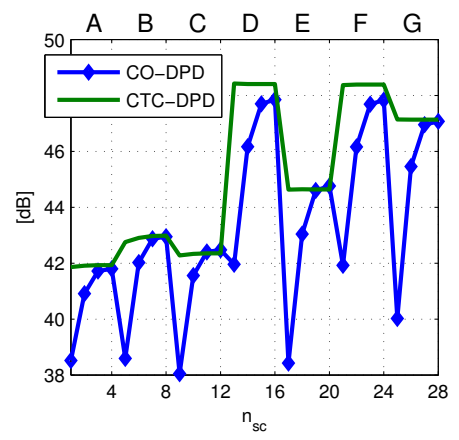

(i)

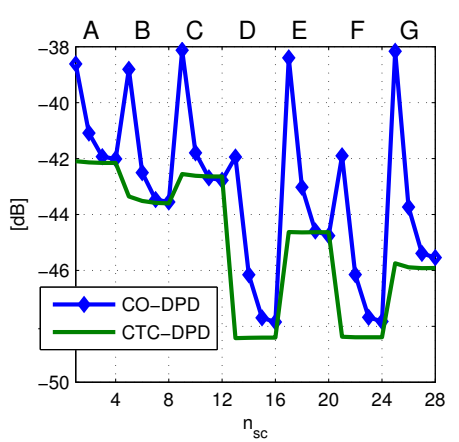

(l)

Fig. 4.5: Simulation results, $N_{t}=2$, LMS-DPD, LMS cross-talk estimation. x-axis: $n_{s c}=4\left(n_{s}-1\right)+n_{c} . n_{s}$ is 1-7 for signals A-G and $n_{c}$ is $1,2,3,4$ for cross-talk levels 54

$-15 \mathrm{~dB},-20 \mathrm{~dB},-30 \mathrm{~dB}$, and no cross-talk. 
Table 4.2.: Comparison of cross-talk estimation methods, $N_{t}=2,-20 \mathrm{~dB}$, Frequency flat cross-talk, signal $\mathrm{B}, \mathrm{PA}_{1}$.

\begin{tabular}{c|c|c|c|c}
\hline $\begin{array}{c}\text { Adaptive } \\
\text { DPD }\end{array}$ & & $\begin{array}{c}\text { Cross-talk } \\
\text { Estimation }\end{array}$ & $\begin{array}{c}\text { NMSE }_{\mathrm{IB}} \\
{[\mathrm{dB}]}\end{array}$ & $\begin{array}{c}\text { ACEPR } \\
{[\mathrm{dB}]}\end{array}$ \\
\hline \hline \multirow{4}{*}{ LMS } & CO-DPD & - & -43.6 & -43.5 \\
\cline { 3 - 5 } & \multirow{2}{*}{ CTC-DPD } & LPF & -41.9 & -43.7 \\
\cline { 3 - 5 } & & LMS & -44.2 & -43.6 \\
\cline { 3 - 5 } & & RLS & -44.3 & -43.7 \\
\hline \multirow{3}{*}{ RLS } & \multirow{2}{*}{ CO-DPD } & - & -42.2 & -43.6 \\
\cline { 3 - 5 } & \multirow{2}{*}{ CTC-DPD } & LPF & -41.1 & -43.9 \\
\cline { 3 - 5 } & & LMS & -42.9 & -44.1 \\
\cline { 3 - 5 } & & RLS & -43.3 & -44.1 \\
\hline
\end{tabular}

malized Mean Squared Error (NMSE $\mathrm{IB}$ ), Adjacent Channel Leakage Ratio (ACLR) and Adjacent Channel Error Power Ratio (ACEPR), which are defined in chapter 3, and the instantaneous error energy. The instantaneous error energy $\xi(n)$ is defined as

$$
\begin{gathered}
\epsilon_{1}(n)=s_{1}(n)-y_{1}(n), \epsilon_{2}(n)=s_{2}(n)-y_{2}(n) \\
\xi_{I}(n)=10 \log \left\{\frac{1}{2}\left(\left|\epsilon_{1}(n)\right|^{2}+\left|\epsilon_{2}(n)\right|^{2}\right)\right\}
\end{gathered}
$$

A moving average filter of window length $L_{w}=25$ samples is applied to visualize the average behavior of $\xi(n)$. The filtered error energy is

$$
\xi(n)=\frac{1}{L_{w}} \sum_{k=-\left(L_{w}-1\right) / 2}^{\left(L_{w}-1\right) / 2} \xi_{I}(n-k)
$$

Fig. 4.2 and Fig. 4.6 shows that the performance of C-DPD degrades at high crosstalk levels, whereas the performances of CO-DPD and CTC-DPD are unaffected by the cross-talk level. NMSE $\mathrm{IB}, \mathrm{ACLR}, \mathrm{EVM}$ and ACEPR are plotted against $n_{s c}$ in Fig. 4.5. $n_{s c}$ which is defined in chapter 3 , is used to index the transmitted signal and the cross-talk level jointly. CTC-DPD performs equally good as CO-DPD for all the examined cases with frequency flat cross-talk. Table 4.2 shows that the linearization performances of CTC-DPD and CO-DPD are almost the same with LMS and RLS algorithms and with different cross-talk estimation methods. This is true for all the cases considered for simulation. We found that the LMS adaptive DPD converges to steady state within 4000 samples (400 $\mu$ s for signal B) and the RLS in 200 samples (20 $\mu s$ for signal B) for all the cases considered, for the particular choice of $\mu, \lambda$ and $\delta$ as specified in Table 4.1. No difference in the convergence behavior is observed between CO-DPD and CTC-DPD. 


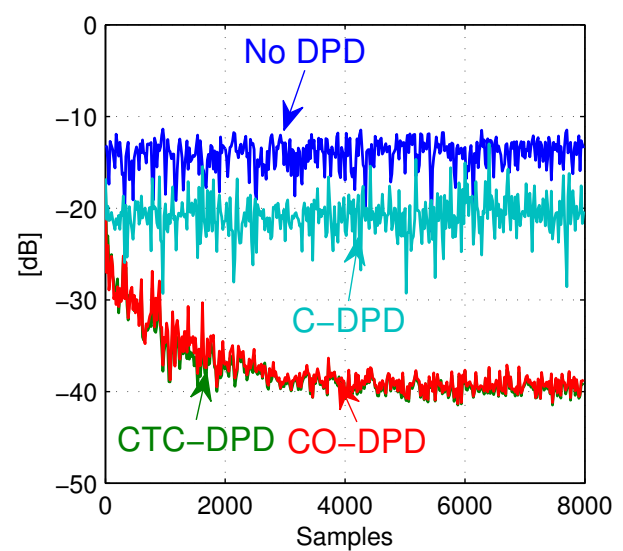

(a) $-15 \mathrm{~dB}$ cross-talk

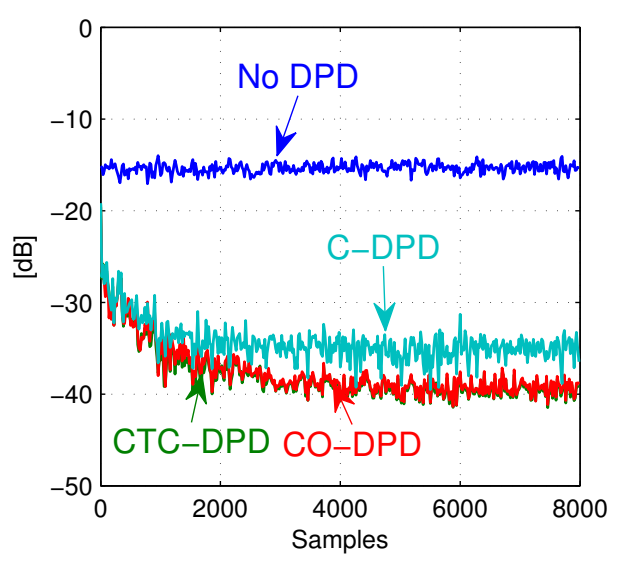

(c) $-30 \mathrm{~dB}$ cross-talk

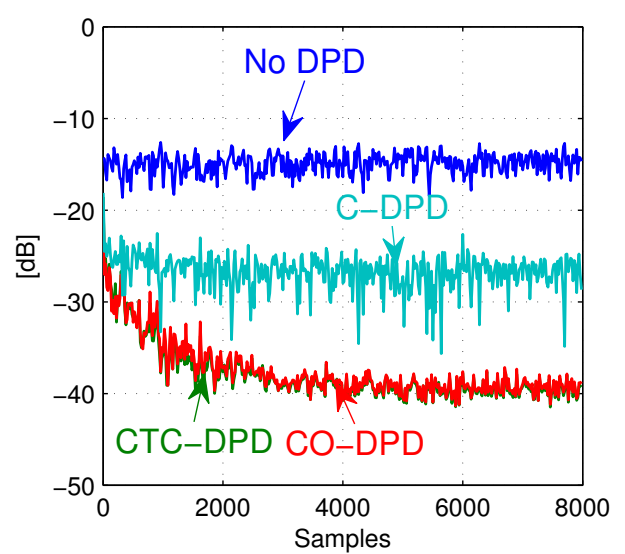

(b) $-20 \mathrm{~dB}$ cross-talk

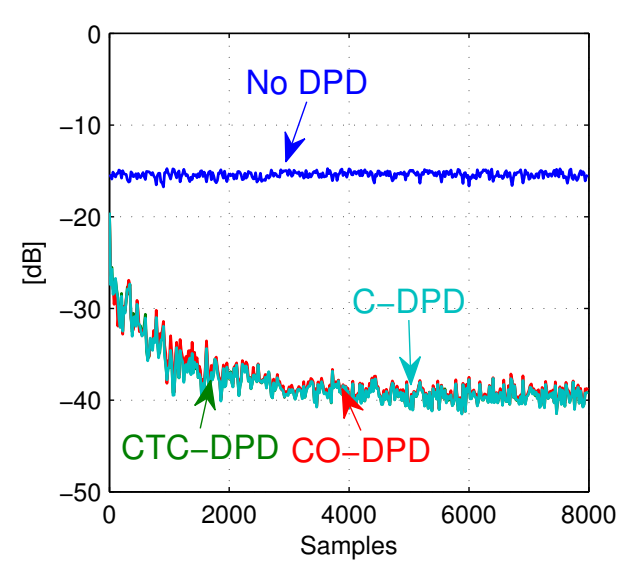

(d) No cross-talk

Fig. 4.6: Filtered error energy $\xi(n), N_{t=2}$, LMS-DPD, signal B, $\mathrm{PA}_{1}$, Frequency flat cross-talk

Fig. 4.5 shows that CTC-DPD performs much better than CO-DPD in the presence of frequency selective cross-talk. The EVM is found to be less than $1.5 \%$ with CTC-DPD for all the examined cases, whereas it is $2-8 \%$ for strong cross-talk levels $(\geq-20 \mathrm{~dB})$ with CO-DPD. We also investigated the effect of the frequency selectivity of the cross-talk on the performances of CO-DPD and CTC-DPD with a QPSK signal (Signal B). We chose six filters whose frequency responses are shown in Fig. 4.7. This is similar to what we have considered in chapter 3. The filters 1a, $2 \mathrm{a}$ and $3 \mathrm{a}$ have the same cutoff frequencies as the signal, i.e. $0.5 \mathrm{MHz}$, and $1 \mathrm{~b}, 2 \mathrm{~b}$ and $3 \mathrm{~b}$ have cutoff frequencies which are twice the cutoff frequency of the signal, i.e. $1 \mathrm{MHz}$. Filters $2 \mathrm{a}$ and $2 \mathrm{~b}$ are Chebyshev filters of order 3 and $3 \mathrm{a}$ and $3 \mathrm{~b}$ are Chebyshev filters of order 6 . The pass-band ripple for all the filters is $1 \mathrm{~dB}$. The EVM and ACLR plots in Fig. 4.8 show that the stronger the frequency selectivity, 


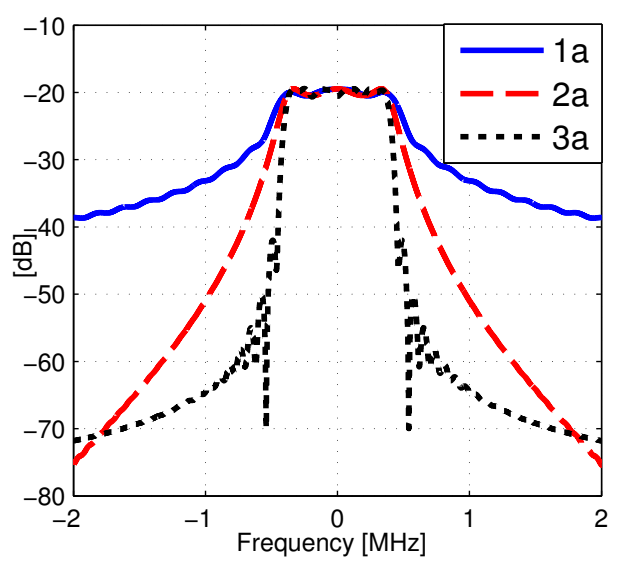

(a) Filters 1a, 2a and 3a

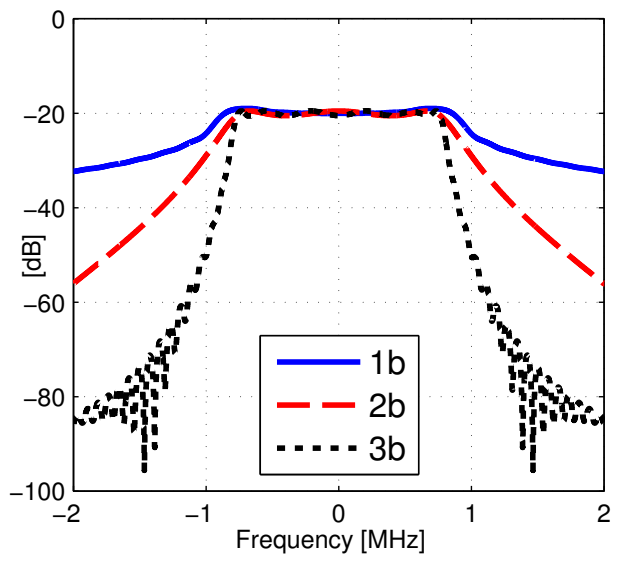

(b) Filters 1b, 2b and 3b

Fig. 4.7: Frequency responses of the coupling filters

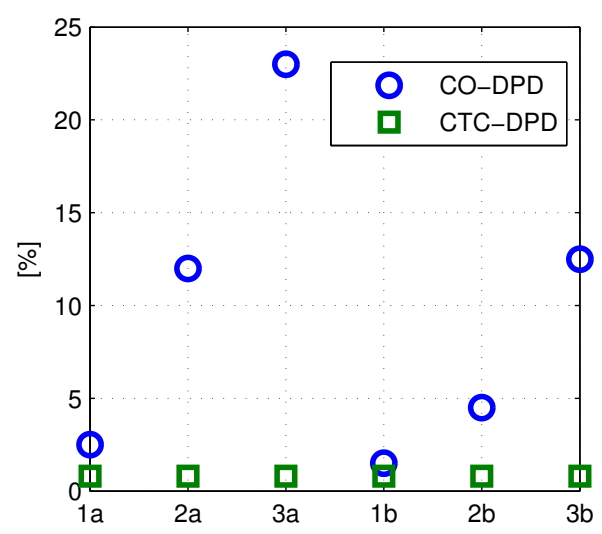

(a) EVM

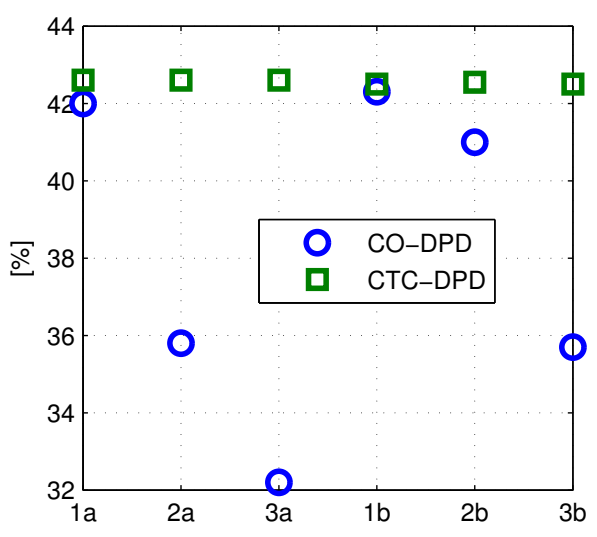

(b) ACLR

Fig. 4.8: Simulation results, $N_{t}=2$, signal $\mathrm{B}, \mathrm{PA}_{1},-20 \mathrm{~dB}$ frequency selective cross-talk 
Table 4.3.: Simulation results, $N_{t}=4$, frequency flat cross-talk, signal B, $\mathrm{PA}_{1}$, LMS-DPD, LMS cross-talk estimation

\begin{tabular}{|c|c|c|c|c|}
\hline \multirow{2}{*}{$\left.\begin{array}{ccc}\beta_{1} & \beta_{2} & \beta_{3}\end{array}\right]$} & \multicolumn{2}{|c|}{$\mathrm{NMSE}_{\mathrm{IB}}[\mathrm{dB}]$} & \multicolumn{2}{|c|}{$\mathrm{ACEPR}[\mathrm{dB}]$} \\
\hline & CO-DPD & CTC-DPD & CO-DPD & CTC-DPD \\
\hline$-20-20-20$ & -42.7 & -43.2 & -43.3 & -43.6 \\
\hline$-20-25-30$ & -42.7 & -43.6 & -43.3 & -43.6 \\
\hline$-20-30-40$ & -42.8 & -43.6 & -43.3 & -43.7 \\
\hline$-30-30-30$ & -42.8 & -43.7 & -43.4 & -43.7 \\
\hline$-30-35-40$ & -42.9 & -43.7 & -43.4 & -43.7 \\
\hline$-30-40-50$ & -42.8 & -43.7 & -43.4 & -43.7 \\
\hline
\end{tabular}

the larger is the improvement achieved by CTC-DPD over CO-DPD, because the CTC-DPD is not affected by the different filter settings, while the performance of the CO-DPD degrades if the frequency selectivity is stronger.

For a four antenna transmitter, we make the following assumption for the cross-talk coefficients

$$
\begin{aligned}
\beta_{12}=\beta_{21}= & \beta_{23}=\beta_{32}=\beta_{43}=\beta_{34}=\beta_{1} \\
\beta_{13}= & \beta_{31}=\beta_{24}=\beta_{42}=\beta_{2} \\
& \beta_{14}=\beta_{41}=\beta_{3} .
\end{aligned}
$$

Simulations are carried out for six cases with different levels of cross-talk. Table 4.3 shows that the linearization performances are almost the same with CO-DPD and CTC-DPD for all the examined cases. 


\section{Chapter 5.}

\section{Complexity Analysis}

In general, the operation of CTC-DPD is computationally less complex compared to CO-DPD, as it involves $N_{t}$ predistorters $\left(N_{t}\right.$ is the number of transmit paths) and the cross-talk pre-cancellation block, whereas the CO-DPD involves $N_{t}^{2}$ predistorters. First, the number of operations (additions, multiplications and divisions) needed for the parameter estimation for a block of $N$ samples and the number of operations needed per sample duration for running the predistorter in real time are counted. In the last section, the computational complexities of the sample-by-sample adaptive algorithms for CO-DPD and CTC-DPD are considered. There are certain operations (e.g. computing the magnitude of the signal values, comparing the signal magnitudes to a threshold, etc.) which we ignore assuming that their computational complexities are negligible compared to additions, multiplications and divisions.

\subsection{Complex and real operations}

All the computations involved in our study require operations on complex numbers. Here, we explain how the complex operations can be realized using real operations. The addition of two complex numbers

$$
(X+i Y)+(U+i V)=(X+U)+i(Y+V)
$$

requires 2 real additions. The multiplication of two complex numbers

$$
\begin{array}{r}
(X+i Y) \times(U+i V)=(X U-Y V)+i(X V+Y U) \\
=(X U-Y V)+i((X+Y) \times(U+V)-X U-Y V)
\end{array}
$$

requires 3 real multiplications and 5 real additions [72]. The division of two complex numbers

$$
\begin{aligned}
& \frac{X+i Y}{U+i V}=\frac{(X+i Y) \times(U-i V)}{U^{2}+V^{2}} \\
& =\frac{(X U+Y V)+i(Y U-X V)}{U^{2}+V^{2}}
\end{aligned}
$$




$$
=\frac{X+Y \times \frac{V}{U}}{U+V \times \frac{V}{U}}+i \frac{Y-X \times \frac{V}{U}}{U+V \times \frac{V}{U}}
$$

requires 3 real divisions, 3 real multiplications and 3 real additions [72]. We denote the number of complex additions, multiplications and divisions used in a certain computation as $N_{c}^{+}, N_{c}^{\times}$and $N_{c}^{\div}$respectively. From (5.1- 5.3), the equivalent number of real additions, multiplications and divisions $N^{+}, N^{\times}$and $N^{\div}$are

$$
\begin{gathered}
N^{+}=2 N_{c}^{+}+5 N_{c}^{\times}+3 N_{c}^{\doteqdot} \\
N^{\times}=3 N_{c}^{\times}+3 N_{c}^{\doteqdot} \\
N^{\div}=3 N_{c}^{\doteqdot}
\end{gathered}
$$

It might be possible to reduce the number of real operations with a different scheme to realize complex operations. However, we assume all the complex operations are realized as in $(5.1-5.3)$, and use (5.4) to convert the number of complex operations to real operations throughout of this chapter.

\subsection{Least squares estimation of DPD}

The least square estimation of the DPD coefficients involves the generation of an $N \times L$ matrix $\boldsymbol{A}_{\boldsymbol{y}}$ and the calculation of $\boldsymbol{d}=\left(\boldsymbol{A}_{\boldsymbol{y}}{ }^{H} \boldsymbol{A}_{\boldsymbol{y}}\right)^{-1} \boldsymbol{A}_{\boldsymbol{y}}{ }^{H} \boldsymbol{x}$, where $\boldsymbol{x}$ is an $N \times 1$ vector and $L=Q(K+1) / 2$ is the size of the DPD coefficient vector $\boldsymbol{d}$. The matrix $\boldsymbol{A}_{\boldsymbol{y}}$ is constructed similar to $\boldsymbol{A}_{\boldsymbol{x}}$ in (3.6). The computation of $a_{k}(y(n))=|y(n)|^{k-1} \cdot y(n)$ requires $k$ real multiplications. So, to compute $\boldsymbol{a}_{\boldsymbol{y}}{ }^{q}$ requires approximately $N((K+1) / 2)^{2}$ real multiplications. To construct $\boldsymbol{A}_{\boldsymbol{y}}=$ $\left[\begin{array}{llllll}\boldsymbol{a}_{\boldsymbol{y}}{ }^{0} & \boldsymbol{a}_{\boldsymbol{y}}{ }^{1} & \ldots & \boldsymbol{a}_{\boldsymbol{y}}{ }^{q} & \ldots & \boldsymbol{a}_{\boldsymbol{y}}{ }^{Q-1}\end{array}\right]$, we need $N((K+1) / 2)^{2} Q$ real multiplications.

$$
N_{A_{y}}^{\times}=N\left(\frac{K+1}{2}\right)^{2} Q
$$

Now, we consider the complexity associated with the computation of $\operatorname{pinv}\left(\boldsymbol{A}_{y}\right)=$ $\left(\boldsymbol{A}_{\boldsymbol{y}}{ }^{H} \boldsymbol{A}_{\boldsymbol{y}}\right)^{-1} \boldsymbol{A}_{\boldsymbol{y}}{ }^{H}$. To multipy $\boldsymbol{A}_{\boldsymbol{y}}$ with its hermitian transpose, we only need $(N-1) L(L+1) / 2$ complex additions and $N L(L+1) / 2$ complex multiplications, if the conjugate symmetry of $\boldsymbol{A}_{\boldsymbol{y}}{ }^{H} \boldsymbol{A}_{\boldsymbol{y}}$ is utilized. The inversion of the $L \times L$ matrix $\boldsymbol{A}_{\boldsymbol{y}}{ }^{H} \boldsymbol{A}_{\boldsymbol{y}}$ require $\left(2 L^{3}+3 L^{2}-5 L\right) / 6$ complex additions, $\left(2 L^{3}+3 L^{2}-5 L\right) / 6$ complex multiplications and $L(L-1) / 2$ complex divisions if the Gaussian elimination method $[73,74]$ is used. Now, this inverse matrix is multiplied with $\boldsymbol{A}_{\boldsymbol{y}}{ }^{H}$ to form the pseudo-inverse $\left(\boldsymbol{A}_{\boldsymbol{y}}{ }^{H} \boldsymbol{A}_{\boldsymbol{y}}\right)^{-1} \boldsymbol{A}_{\boldsymbol{y}}{ }^{H}$. This requires $(L-1) L N$ complex additions 
and $L^{2} N$ complex multiplications

$$
\begin{gathered}
N_{c, \text { pinv }}^{+}(N, L)=\frac{(N-1) L(L+1)}{2}+(L-1) L N+\frac{2 L^{3}+3 L^{2}-5 L}{6} \\
N_{c, \text { pinv }}^{\times}(N, L)=\frac{N L(L+1)}{2}+L^{2} N+\frac{2 L^{3}+3 L^{2}-5 L}{6} \\
N_{c, \text { pinv }}^{\div}(L)=\frac{L(L-1)}{2} .
\end{gathered}
$$

Finally, the multiplication of $\operatorname{pinv}\left(\boldsymbol{A}_{y}\right)$ with $\boldsymbol{x}$ to compute the DPD coefficients, requires $L(N-1)$ complex additions and $L N$ complex multiplications.

$$
\begin{gathered}
N_{c, \mathrm{LS}}^{+}(N, L)=N_{c, \text { pinv }}^{+}(N, L)+L(N-1) \\
N_{c, \mathrm{LS}}^{\times}(N, L)=N_{c, \text { pinv }}^{\times}(N, L)+L N \\
N_{c, \mathrm{LS}}^{\dot{亠}}(L)=N_{c, \text { pinv }}^{\dot{亠}}(L) .
\end{gathered}
$$

\subsection{Estimation of coupling coefficients for CTC-DPD}

The methods for cross-talk estimation are explained in section 3.2. The numbers of computations required for the least squares estimation of the cross-talk coefficients for CTC-DPD for a two antenna transmitter are

$$
\begin{aligned}
N_{c, \hat{\alpha}}^{+}= & N_{c, \mathrm{LS}}^{+}\left(N_{1}, M+1\right)+N_{c, \mathrm{LS}}^{+}\left(N_{2}, M+1\right) \\
N_{c, \hat{\alpha}}^{\times}= & N_{c, \mathrm{LS}}^{\times}\left(N_{1}, M+1\right)+N_{c, \mathrm{LS}}^{\times}\left(N_{2}, M+1\right) \\
& N_{c, \hat{\alpha}}^{\leftarrow}=2 N_{c, \mathrm{LS}}^{\div}(M+1)+2 M,
\end{aligned}
$$

where $M$ is the number of filter taps that model the cross-talk. (5.8) can be applied to frequency flat $(M=1)$ and frequency selective cross-talk $(M>1) . N_{1}$ and $N_{2}$ are the lengths of $\tilde{\boldsymbol{y}}_{1}$ and $\check{\boldsymbol{y}}_{2}$ respectively, which are constructed by removing the elements from the output signal vectors corresponding to the PA nonlinear region. The maximum value of $N_{1}$ or $N_{2}$ among all of our measurements with $T=0.3$ (threshold for cross-talk estimation) using different signals and PA responses was found to be $0.17 N$. So, in this section, we use $N_{1}=N_{2}=0.17 N$ in order to have a reasonable estimate of the complexity associated with the estimation of coupling coefficients. To apply CTC-DPD, we need to invert the estimate of cross-talk matrix in the case of frequency flat cross-talk. The numbers of computations needed for this operation are

$$
\begin{aligned}
& N_{c, \text { inv } \alpha}^{+}=1 \\
& N_{c, \text { inv } \alpha}^{\times}=1 \\
& N_{c, \text { inv } \alpha}^{\doteqdot}=2 .
\end{aligned}
$$

In the case of frequency selective cross-talk, $c_{k}$ in (3.34) need to be computed. The numbers of computations needed are 


$$
\begin{gathered}
N_{c, \text { inv } \alpha}^{+}=M^{2} \\
N_{c, \text { inv } \alpha}^{\times}=(M-1)^{2} \\
N_{c, \text { inv } \alpha}^{\doteqdot}=0 .
\end{gathered}
$$

\subsection{Memory polynomial DPD}

To apply the memory polynomial predistorter on the input signal according to (2.12), we need $Q((K+1) / 2)^{2}$ real multiplications, $Q((K+1) / 2)$ complex multiplications and $(Q-1)(K-1) / 2$ complex additions per sample. The equivalent numbers of real operations are

$$
\begin{gathered}
N_{\mathrm{DPD}}^{+}=(Q-1)(K-1)+5 Q\left(\frac{K+1}{2}\right) \\
N_{\mathrm{DPD}}^{\times}=3 Q\left(\frac{K+1}{2}\right)+Q\left(\frac{K+1}{2}\right)^{2} \\
N_{\mathrm{DPD}}^{\dot{ }}=0 .
\end{gathered}
$$

\subsection{Cancelling the cross-talk}

The numbers of operations per sample required for the cancellation of frequency flat cross-talk for a two antenna transmitter according to (3.13)

$$
\begin{aligned}
& N_{c, \alpha^{-1}}^{+}=2 \\
& N_{c, \alpha^{-1}}^{\times}=4 \\
& N_{c, \alpha^{-1}}^{\dot{\div}}=0 .
\end{aligned}
$$

The numbers of complex operations per sample, needed for the cancellation of frequency selective cross-talk as explained in ((3.31) are

$$
\begin{aligned}
& N_{c, \alpha^{-1}}^{+}=6 M-6 \\
& N_{c, \alpha^{-1}}^{\times}=6 M-6 \\
& N_{c, \alpha^{-1}}^{\dot{\div}}=4 .
\end{aligned}
$$


Table 5.1.: Comparison of complexity, $N_{t}=2, K=9, Q=3, M=1$

\begin{tabular}{c|c|c|c}
\hline \multicolumn{2}{c|}{} & CO-DPD & CTC-DPD \\
\hline \hline \multicolumn{2}{c|}{$N^{+}$} & 368 & 206 \\
\hline \multicolumn{2}{c|}{$N^{\times}$} & 480 & 252 \\
\hline \multirow{4}{*}{$N_{\text {Est }}^{+}$} & $N=10^{3}$ & $9.9 \times 10^{6}$ & $5 \times 10^{6}$ \\
\cline { 2 - 4 } & $N=10^{4}$ & $9.9 \times 10^{7}$ & $5 \times 10^{7}$ \\
\cline { 2 - 4 } & $N=10^{5}$ & $9.9 \times 10^{8}$ & $5 \times 10^{8}$ \\
\hline \multirow{4}{*}{$N_{\text {Est }}^{\times}$} & $N=10^{3}$ & $4.5 \times 10^{6}$ & $2.3 \times 10^{6}$ \\
\cline { 2 - 4 } & $N=10^{4}$ & $4.4 \times 10^{7}$ & $2.3 \times 10^{7}$ \\
\cline { 2 - 4 } & $N=10^{5}$ & $4.4 \times 10^{8}$ & $2.3 \times 10^{8}$ \\
\hline \multirow{2}{*}{} & $N_{\text {Est }}^{\dot{*}}$ & 1305 & 645 \\
\hline
\end{tabular}

\subsection{Comparing the complexity of CO-DPD and CTC-DPD}

In this section we compare the computational complexities of CTC-DPD and CODPD for a MIMO tranmistter with $N_{t}$ antennas. The least squares estimation of the CO-DPD coefficients includes the computation of the pseudo-inverse of $\boldsymbol{A}_{y}=$ $\left[\begin{array}{lll}\boldsymbol{A}_{y_{1}} & \boldsymbol{A}_{y_{2}} \ldots & \boldsymbol{A}_{y_{N_{t}}}\end{array}\right]$, an $N \times N_{t} L$ matrix, and the multiplication with $\boldsymbol{x}=\left[\begin{array}{ll}\boldsymbol{x}_{1} & \boldsymbol{x}_{2} \ldots\end{array}\right.$ $\left.\ldots \boldsymbol{x}_{N_{t}}\right]$, an $N \times N_{t}$ matrix. The numbers of complex operations needed are

$$
\begin{gathered}
N_{c, \text { Est }}^{+}=N_{c, \text { pinv }}^{+}\left(N, N_{t} L\right)+N_{t}^{2} L(N-1) \\
N_{c, \text { Est }}^{\times}=N_{c, \text { pinv }}^{\times}\left(N, N_{t} L\right)+N_{t}^{2} L N \\
N_{c, \text { Est }}^{\dot{ }}=N_{c, \text { pinv }}^{\doteqdot}\left(N_{t} L\right) .
\end{gathered}
$$

The identification of parameters for CTC-DPD includes the estimation of two DPD coefficient vectors, and the cross-talk coefficients. The numbers of complex operations needed are

$$
\begin{gathered}
N_{c, \text { Est }}^{+}=N_{t} N_{c, \text { pinv }}^{+}(N, L)+N_{t} L(N-1)+N_{c, \hat{\alpha}}^{+}+N_{c, \text { inv } \alpha}^{+} \\
N_{c, \text { Est }}^{\times}=N_{t} N_{c, \text { pinv }}^{\times}(N, L)+N_{t} L N+N_{c, \hat{\alpha}}^{\times}+N_{c, \text { inv } \alpha}^{\times} \\
N_{c, \text { Est }}^{\dot{\bullet}}=N_{t} N_{c, \text { pinv }}^{\dot{\doteqdot}}(L)+N_{c, \hat{\alpha}}^{\dot{-}}
\end{gathered}
$$

The total number of real operations for CO-DPD and CTC-DPD are obtained using (5.4) and adding the number of real multiplications needed to generatete $\boldsymbol{A}_{y_{1}}, \boldsymbol{A}_{y_{2}} \ldots$ $\boldsymbol{A}_{y_{N_{t}}}$ matrices, i.e. $N_{t} N_{A_{y}}^{\times}$. The numbers of real operations per sample, needed for running the CO-DPD — which contains $N_{t}^{2}$ branch predistorters - are 
Table 5.2.: Complexity ratio (\%), $N_{t}=2, N=10^{3}$

\begin{tabular}{c|c|c|c|c|c|c|c}
\hline \multicolumn{2}{c|}{$\begin{array}{c}\text { Cross-talk } \\
\rightarrow\end{array}$} & \multicolumn{2}{c|}{$\begin{array}{c}\text { Frequency- } \\
\text { flat }(M=1)\end{array}$} & \multicolumn{4}{c}{$\begin{array}{c}\text { Frequency- } \\
\text { selective, } Q=3\end{array}$} \\
\hline \multirow{2}{*}{$\mathrm{K}=3$} & $\mathrm{Q}=1$ & $\mathrm{Q}=3$ & $\mathrm{M}=2$ & $\mathrm{M}=5$ & $\mathrm{M}=10$ & $\mathrm{M}=100$ \\
\cline { 3 - 8 } & $\eta_{\text {run }}$ & 80 & 60 & 75 & 120 & 195 & 1545 \\
\cline { 2 - 8 } & $\eta_{\text {est }}$ & 64.7 & 53.9 & 55.1 & 61.2 & 79.6 & 2397 \\
\hline \multirow{2}{*}{$\mathrm{K}=5$} & $\eta_{\text {run }}$ & 66.7 & 55.6 & 63.9 & 88.9 & 130.6 & 880.5 \\
\cline { 2 - 8 } & $\eta_{\text {est }}$ & 60.1 & 52.9 & 53.5 & 56.3 & 64.8 & 1135.5 \\
\hline \multirow{2}{*}{$\mathrm{K}=7$} & $\eta_{\text {run }}$ & 60.7 & 53.6 & 58.9 & 75 & 101.8 & 583.9 \\
\cline { 2 - 8 } & $\eta_{\text {est }}$ & 58.2 & 52.5 & 52.8 & 54.4 & 59.3 & 673.4 \\
\hline \multirow{2}{*}{$\mathrm{K}=9$} & $\eta_{\text {run }}$ & 57.5 & 52.5 & 56.2 & 67.5 & 86.2 & 423.7 \\
\cline { 2 - 8 } & $\eta_{\text {est }}$ & 57.2 & 52.2 & 52.5 & 53.5 & 56.6 & 454.1 \\
\hline \multirow{2}{*}{$\mathrm{K}=11$} & $\eta_{\text {run }}$ & 55.6 & 51.8 & 54.6 & 62.9 & 76.8 & 326.8 \\
\cline { 2 - 7 } & $\eta_{\text {est }}$ & 56.6 & 52.1 & 52.3 & 52.9 & 55.2 & 333.2 \\
\hline
\end{tabular}

Table 5.3.: Complexity ratio $\eta[\%], M=1, Q=3, N=10^{3}$

\begin{tabular}{c|c|c|c|c|c|c|c|c}
\hline \multicolumn{2}{c|}{$N_{t} \rightarrow$} & 2 & 3 & 4 & 5 & 6 & 7 & 8 \\
\hline \hline \multirow{2}{*}{$\mathrm{K}=3$} & $\eta_{\text {run }}$ & 60 & 43.3 & 35 & 30 & 26.7 & 24.3 & 22.5 \\
\cline { 2 - 9 } & $\eta_{\text {est }}$ & 53.9 & 37.4 & 29.1 & 24.2 & 21.1 & 18.9 & 17.4 \\
\hline \multirow{2}{*}{$\mathrm{K}=5$} & $\eta_{\text {run }}$ & 55.6 & 38.9 & 30.6 & 25.5 & 22.2 & 19.8 & 18.1 \\
\cline { 2 - 9 } & $\eta_{\text {est }}$ & 52.9 & 36.2 & 27.7 & 22.6 & 19.3 & 16.9 & 15.2 \\
\hline \multirow{2}{*}{$\mathrm{K}=7$} & $\eta_{\text {run }}$ & 53.6 & 36.9 & 28.6 & 23.6 & 20.2 & 17.9 & 16.1 \\
\cline { 2 - 9 } & $\eta_{\text {est }}$ & 52.5 & 35.6 & 27.1 & 21.9 & 18.5 & 16.1 & 14.3 \\
\hline \multirow{2}{*}{$\mathrm{K}=9$} & $\eta_{\text {run }}$ & 52.5 & 35.8 & 27.5 & 22.5 & 19.2 & 16.8 & 15 \\
\cline { 2 - 9 } & $\eta_{\text {est }}$ & 52.2 & 35.4 & 26.8 & 21.6 & 18.1 & 15.7 & 13.8 \\
\hline \multirow{2}{*}{$\mathrm{K}=11$} & $\eta_{\text {run }}$ & 51.8 & 35.2 & 26.8 & 21.8 & 18.5 & 16.1 & 14.4 \\
\cline { 2 - 8 } & $\eta_{\text {est }}$ & 52.1 & 35.2 & 26.8 & 21.4 & 17.9 & 15.4 & 13.6
\end{tabular}




$$
\begin{aligned}
& N^{+}=N_{t}^{2} N_{D P D}^{+}+2 N_{t} \\
& N^{\times}=N_{t}^{2} N_{D P D}^{\times} \\
& N^{\doteqdot}=0 .
\end{aligned}
$$

The $2 N_{t}$ extra adders come from the $N_{t}$ complex adders needed to combine the output signals of the branches of CO-DPD. The numbers of real operations per sample, needed for running the CTC-DPD - which contains $N_{t}$ branch predistorters - are

$$
\begin{gathered}
N^{+}=N_{t} N_{D P D}^{+}+N_{\alpha^{-1}}^{+} \\
N^{\times}=N_{t} N_{D P D}^{\times}+N_{\alpha^{-1}}^{\times} \\
N^{\doteqdot}=N_{\alpha^{-1}}^{\doteqdot} .
\end{gathered}
$$

In section 5.3 and section 5.5, the numbers of computations needed for the estimation of coupling coefficients $\left(N_{c, \hat{\alpha}}\right)$, computing its inverse filter coefficients $\left(N_{c, \text { inv } \alpha}\right)$ and cancelling the cross-talk $\left(N_{\alpha^{-1}}\right)$ for CTC-DPD are computed for a two antenna transmitter. These quantities can be computed for any number of antennas in similar ways.

The numbers of real operations corresponding to (5.14 - 5.17) needed for CO-DPD and CTC-DPD for a two antenna transmitter, with a memory polynomial order $K=9$ and memory depth $Q=3$, for frequency flat cross-talk $(M=1)$ are given in Table 5.1. We observe that the number of computations needed for CTC-DPD is approximately half that of CO-DPD. This is expected as the CO-DPD structure uses two times the number of DPD branches compared to CTC-DPD. We can further note that the number of divisions needed is much smaller compared to the number of additions and multiplications. We can assume that the addition operation is much simpler compared to multiplication. So, in order to assess the computational complexities of the MIMO-DPD schemes, it is sufficient to compare the number of multiplications. Now we define two complexity ratios $\eta_{\text {run }}$ and $\eta_{\text {est }}$ as the ratios of the number of multiplications needed for CTC-DPD and CO-DPD.

$$
\begin{aligned}
\eta_{\text {run }} & =\frac{N^{\times}(\mathrm{CTC}-\mathrm{DPD})}{N^{\times}(\mathrm{CO}-\mathrm{DPD})} \times 100 \% \\
\eta_{\text {est }} & =\frac{N_{E s t}^{\times}(\mathrm{CTC}-\mathrm{DPD})}{N_{E s t}^{\times}(\mathrm{CO}-\mathrm{DPD})} \times 100 \% .
\end{aligned}
$$

$\eta_{\text {run }}$ and $\eta_{\text {est }}$ indicate the fraction of computations needed for CTC-DPD compared to CO-DPD. The values of $\eta_{\text {run }}$ and $\eta_{\text {est }}$ for different memory polynomial orders $(K)$ and memory depths $(Q)$ for frequency flat cross-talk and frequency selective cross-talk are given in Table 5.2. CTC-DPD reduces the computational complexity considerably compared to CO-DPD. For larger memory polynomial orders (e.g. $K \geq$ $9), \eta_{\text {run }}$ and $\eta_{\text {est }}$ are in the range of $50-60 \%$. This is expected for a two antenna transmitter, as the CO-DPD is a 4-branch structure, whereas CTC-DPD contains only 2 branches and an additional cross-talk pre-cancellation block. 
Table 5.4.: Comparison of complexity of adaptive MIMO predistortion, $K=9$, $Q=3, M=1$

\begin{tabular}{c|c|c|c|c|c|c}
\hline & \multicolumn{2}{|c|}{$N_{t}=2$} & \multicolumn{2}{c|}{$N_{t}=4$} & \multicolumn{2}{c}{$N_{t}=8$} \\
\hline $\mathrm{DPD} \rightarrow$ & CO & CTC & CO & CTC & CO & CTC \\
\hline \hline$N^{+}$ & 1156 & 639 & 4600 & 1628 & 18376 & 4984 \\
\hline$N^{\times}$ & 1260 & 664 & 5040 & 1500 & 20160 & 3832 \\
\hline$N^{\div}$ & 0 & 3 & 0 & 18 & 0 & 84 \\
\hline
\end{tabular}

Table 5.5.: Complexity ratio $\eta[\%]$, adaptive MIMO predistortion, $N_{t}=2$

\begin{tabular}{c|c|c|c|c|c|c}
\hline $\begin{array}{c}\text { Cross-talk } \\
\rightarrow\end{array}$ & \multicolumn{2}{|c|}{$\begin{array}{c}\text { Frequency- } \\
\text { flat }(\mathrm{M}=1)\end{array}$} & \multicolumn{4}{c}{$\begin{array}{c}\text { Frequency- } \\
\text { selective, Q=3 }\end{array}$} \\
\hline & $\mathrm{Q}=1$ & $\mathrm{Q}=3$ & $\mathrm{M}=2$ & $\mathrm{M}=5$ & $\mathrm{M}=10$ & $\mathrm{M}=100$ \\
\hline $\mathrm{K}=3$ & 78.3 & 59.4 & 70.6 & 116.4 & 226.1 & 9326.1 \\
\hline $\mathrm{K}=5$ & 66.7 & 55.6 & 62.1 & 89.1 & 153.6 & 5506.5 \\
\hline $\mathrm{K}=7$ & 61.2 & 53.7 & 58.1 & 76.2 & 119.5 & 3711.6 \\
\hline $\mathrm{K}=9$ & 58.1 & 52.7 & 55.9 & 68.9 & 100.3 & 2700.3 \\
\hline $\mathrm{K}=11$ & 56.2 & 52.1 & 54.5 & 64.4 & 88.3 & 2066.5 \\
\hline
\end{tabular}

If CO-DPD can provide the same linearization performance with lower order and memory for the cross DPD branches, the complexity of CO-DPD would be reduced and $\eta_{\text {est }}$ and $\eta_{\text {run }}$ would become higher. If the complexity of the least square estimation or the real time operation of the DPDs can be reduced, $\eta_{\text {est }}$ and $\eta_{\text {run }}$ increase respectively, because the complexity reduction in such cases would be higher for CO-DPD. E.g, it is possible to reduce $N_{D P D}^{\times}$using look-up tables [34,75]. The complexity of CTC-DPD for frequency selective cross-talk increases as the number of filter coefficients $(M)$ that model the cross-talk increases. For higher number of transmit paths $\left(N_{t}>2\right), \eta_{\text {est }}$ and $\eta_{\text {run }}$ becomes even lower, as can be seen from Table 5.3.

\subsection{Complexity of adaptive MIMO predistortion}

In this section, we compare the computational complexities of the considered adaptive algorithms for CO-DPD and CTC-DPD for a two-antenna transmitter. We follow the same method and assumptions as used in the previous sections. The number of operations needed for adaptive CO-DPD is counted by adding up the 
Table 5.6.: Complexity Ratio $\eta[\%]$, adaptive MIMO predistortion, $M=1, Q=3$

\begin{tabular}{c|c|c|c|c|c|c|c}
\hline$N_{t} \rightarrow$ & 2 & 3 & 4 & 5 & 6 & 7 & 8 \\
\hline \hline $\mathrm{K}=3$ & 59.4 & 47.8 & 41.7 & 38.4 & 36.7 & 35.7 & 35.3 \\
\hline $\mathrm{K}=5$ & 55.6 & 41.8 & 34.8 & 30.8 & 28.4 & 26.9 & 25.9 \\
\hline $\mathrm{K}=7$ & 53.7 & 39.1 & 31.6 & 27.3 & 24.6 & 22.7 & 21.5 \\
\hline $\mathrm{K}=9$ & 52.7 & 37.5 & 29.8 & 25.3 & 22.4 & 20.4 & 19 \\
\hline $\mathrm{K}=11$ & 52.1 & 36.5 & 28.6 & 24.1 & 21.1 & 18.9 & 17.5 \\
\hline
\end{tabular}

number of operations needed for running the $N_{t}^{2}$ branch predistorters and postdistorters and updating the DPD coefficients using the LMS/RLS algorithms. The number of operations needed for adaptive CTC-DPD is counted by adding up the number of operations needed for running the $N_{t}$ predistorters and post-distorters, updating the DPD coefficients, canceling the cross-talk and updating the estimate of the coupling coefficients. Similar to the previous section, a complexity ratio $\eta$ can be defined as the ratio of the number of multiplications needed for CTC-DPD and CO-DPD

$$
\eta=\frac{N^{\times}(\mathrm{CTC}-\mathrm{DPD})}{N^{\times}(\mathrm{CO}-\mathrm{DPD})} \times 100 \%
$$

The complexity ratio for different memory polynomial orders $(K)$, memory depths $(Q)$ and number of transmit paths $\left(N_{t}\right)$ are given in Table 5.5 and Table 5.6. These values of $\eta$ are derived for LMS adaptive DPD and LMS cross-talk estimation. For RLS adaptive DPD and other cross-talk estimation methods described in chapter 4, the complexity ratio shows similar behavior. 


\section{Chapter 6.}

\section{Conclusions}

In this thesis, we discussed predistortion architectures for PAs in multiple antenna transmitters which consider the cross-talk before the PA. As this cross-talk is passed through the nonlinearity of the PAs this is called non-linear crosstalk. In the presence of such non-linear cross-talk in MIMO transmitters, the performance of the conventional DPD (C-DPD) algorithms degrades considerably. Two architecturesCO-DPD which was proposed in [8] and CTC-DPD which we proposed in [9] - to pre-compensate the non-linear cross-talk and PA non-linearity are considered in this thesis.

The least squares (LS) method of DPD identification can be extended for CO-DPD. We applied the LS method for the identification of the DPD coeffcients and the coupling factors which model the cross-talk in CTC-DPD. The CTC-DPD structure can be extended to pre-cancel the frequency selective cross-talk, while CO-DPD can not. The linearization performance of CTC-DPD is compared with CO- DPD using performance measures such as In-band NMSE, EVM, ACLR and ACEPR. Measurements were carried out in a block-by-block manner, for a two antenna transmitter, using QPSK, 16-QAM, UMTS and LTE signals with transmission bandwidths varying from $1 \mathrm{MHz}$ to $10 \mathrm{MHz}$ and PAPRs varying from $3.4 \mathrm{~dB}$ to $7.2 \mathrm{~dB}$ for two different PA responses. We also applied the recursive least squares (RLS) and least mean squared (LMS) adaptive predistortion algorithms for CTC-DPD. The linearization performance of adaptive algorithms for CTC-DPD is compared with CO-DPD using simulations. The PA responses used in the simulations are estimated from the input and output signal samples obtained from measurements.

The CTC-DPD achieves a slight linearization performance improvement over CODPD at strong frequency flat cross-talk levels, when the PA is operated with high non-linearity with signals having low peak-to-average-power ratios (PAPR), and performs approximately identical to CO-DPD in other cases. It is observed that CTC-DPD achieves $0.6 \%$ improvement in EVM $(1.8 \mathrm{~dB}$ improvement in inband NMSE) at $-15 \mathrm{~dB}$ cross-talk compared to CO-DPD, for signal $\mathrm{A}$, at $\mathrm{PEP}_{\text {in }}=$ $10 \mathrm{dBm}$. In the presence of frequency selective cross-talk, CTC-DPD performs considerably better than CO-DPD which can not model such cross-talk.

The computational complexities of these MIMO-DPD schemes for the block-based and adaptive operations are assessed by counting the number of additions, multi- 
plications and divisions needed for the parameter identification and the real time operation. It is found that the computational complexity is mainly determined by the number of multiplications required. The computational complexity of CTCDPD for a two-antenna transmitter is estimated to be $46 \%$ lesser compared to CODPD, when a $9^{\text {th }}$ order memory polynomial with 3 memory taps was used. This is because the CO-DPD is a 4-branch structure, whereas CTC-DPD contains only 2 branches and an additional cross-talk pre-cancellation block, for a two antenna transmitter. The complexity reduction achieved by CTC-DPD over CO-DPD becomes higher as the number of transmit paths increases. Because of these reasons CTC-DPD becomes the ideal candidate for a predistorter in MIMO transmitters with non-linear RF cross-talk.

The pre-compensation of I/Q imbalance and DC offset along with the cross-talk and PA nonlinearity, in MIMO transmitters are topics for further study. An extension of CO-DPD which also takes in to account the I/Q imbalance and DC offset was presented in [63]. The coupling and other RF impairments present in the feedback receiver paths are expected to affect the predistortion for multiple antenna transmitters. The techniques to mitigate these effects are also interesting topics for further study.

Massive MIMO wireless communications refers to the use of very large number of antennas, and can potentially improve the spectral and energy efficiency by orders of magnitude compared to single antenna systems [76]. Digital signal processing techniques for RF impairment mitigation for the massive MIMO systems would be another interesting area for future research. 



\section{Appendix A.}

\section{Measurement results (Two antenna Tx)}

This section contains the detailed information about the measurement results, which are discussed in chapter 3. We define the differences in the performance measures between CO-DPD and CTC-DPD

$$
\begin{gathered}
\Delta \mathrm{NMSE}_{\mathrm{IB}}=\mathrm{NMSE}_{\mathrm{IB}}(\mathrm{CO})-\mathrm{NMSE}_{\mathrm{IB}}(\mathrm{CTC}) \\
\Delta \mathrm{EVM}=\operatorname{EVM}(\mathrm{CO})-\operatorname{EVM}(\mathrm{CTC}) \\
\Delta \mathrm{ACLR}=\operatorname{ACLR}(\mathrm{CO})-\operatorname{ACLR}(\mathrm{CTC}) \\
\Delta \mathrm{ACEPR}=\operatorname{ACEPR}(\mathrm{CO})-\operatorname{ACEPR}(\mathrm{cTC})
\end{gathered}
$$

These quantities are plotted against an indexing variable $n_{s c}$ in Fig. A.1. $n_{s c}$ jointly represents the transmitted signal and the cross-talk level, and is defined in (3.51). The details of the transmit signals are given in Table 3.2. The performance measures such as NMSE $\mathrm{IB}_{\mathrm{IB}}$ EVM, ACLR, ACEPR are plotted against $n_{s c}$ in Fig. A.2. Fig. A.3 shows $\varepsilon_{L S}, \varepsilon_{\text {Leak }}$, average output power $\left(\mathrm{P}_{\text {out }}\right)$ and peak output power $\left(\mathrm{PEP}_{\text {out }}\right)$ of the PA.

Fig. A.1d and Fig. A.1a show that there is improvement for CTC-DPD in NMSE $\mathrm{IB}_{\mathrm{B}}$ and EVM compared to CO-DPD, with frequency flat cross-talk at $\mathrm{PEP}_{\text {in }}=10 \mathrm{dBm}$, especially at $n_{s c}=1,13,21$ corresponding to signals $\mathrm{A}, \mathrm{D}$ and $\mathrm{F}$ at $-15 \mathrm{~dB}$ cross-talk. $\triangle \mathrm{ACLR}$ and $\triangle \mathrm{ACEPR}$ are negligibly small for all the $n_{s c}$ values at $\mathrm{PEP}_{\text {in }}=10 \mathrm{dBm}$. Figures A.1e, A.1b, A.1h and A.1k show that $\triangle \mathrm{NMSE}_{\mathrm{IB}}, \triangle \mathrm{EVM}, \triangle \mathrm{ACLR}$ and $\triangle \mathrm{ACEPR}$ are negligibly small for all $n_{s}$ with frequency flat cross-talk at $\mathrm{PEP}_{\text {in }}=$ $7 \mathrm{dBm}$. Figures A.1f, A.1c, A.1i and A.1l show noticeable improvement for CTCDPD in NMSE $\mathrm{IB}_{\mathrm{IB}}, \mathrm{EVM}, \mathrm{ACLR}$ and ACEPR with frequency selective cross-talk at $\mathrm{PEP}_{\text {in }}=7 \mathrm{dBm}$. Figures A.3d and A.3e show that $\varepsilon_{L S}$ is the same for CO-DPD and CTC-DPD with frequency flat cross-talk, for all $n_{s c}$ values. Fig. A.3f shows that $\varepsilon_{L S}$ is slightly higher for $n_{s c}$ values corresponding to $-15 \mathrm{~dB}$ frequency selective crosstalk. Figures A.3g and A.3h show that the $\varepsilon_{\text {Leak }}$ values are relatively higher for CO-DPD with frequency flat cross-talk, at $k=2$, especially for $n_{s c}=1,13,21$ corresponding to signals $\mathrm{A}, \mathrm{D}$ and $\mathrm{F}$ at $-15 \mathrm{~dB}$ cross-talk. $\varepsilon_{\text {Leak }}$ is smaller for $\mathrm{PEP}_{\text {in }}=7 \mathrm{dBm}$ compared to $\mathrm{PEP}_{\text {in }}=10 \mathrm{dBm}$. Fig. A.3i shows that $\varepsilon_{\text {Leak }}$ is higher for CO-DPD with frequency selective cross-talk, for all $k$. 
The behavior of these performance measures for CO-DPD and CTC-DPD for different cases, and the reasons are explained in chapter 3 . This section contains the complete set of measrement results, of which only a relevant part is presented in chapter 3 . 


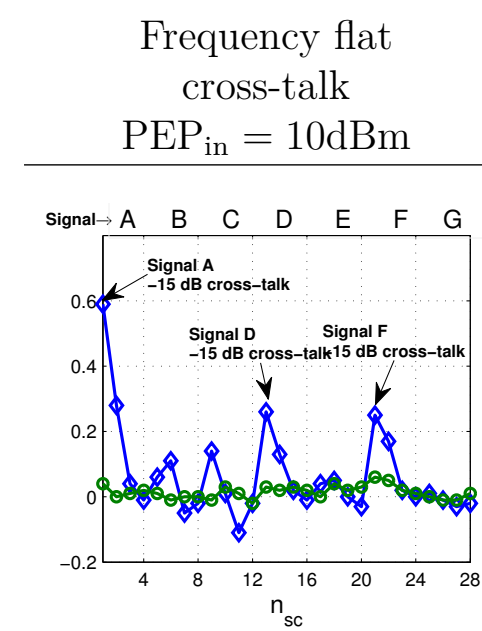

(a) $\Delta \mathrm{EVM}[\%]$

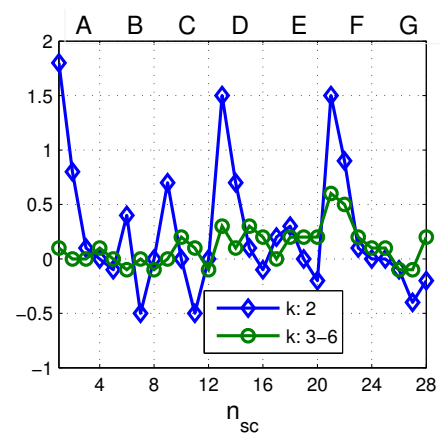

(d) $\Delta \mathrm{NMSE}_{\mathrm{IB}}[\mathrm{dB}]$

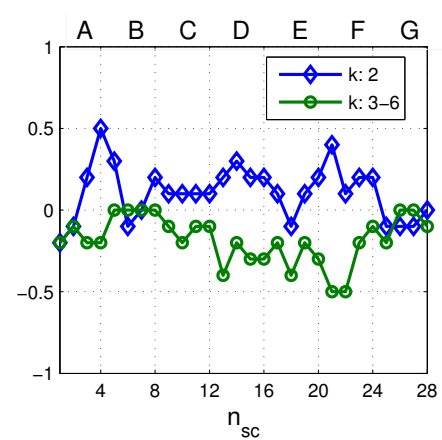

(g) $\triangle \mathrm{ACLR}[\mathrm{dB}]$

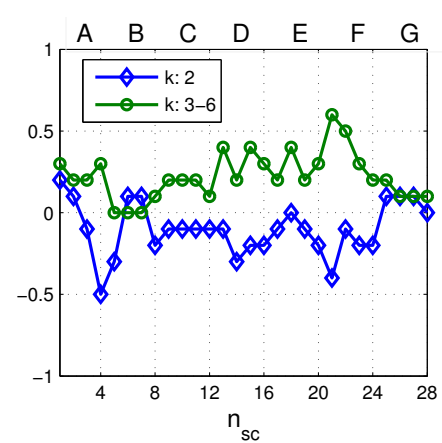

(j) $\triangle \mathrm{ACEPR}[\mathrm{dB}]$
Frequency flat cross-talk

$\mathrm{PEP}_{\text {in }}=7 \mathrm{dBm}$
Frequency selective cross-talk $\mathrm{PEP}_{\text {in }}=7 \mathrm{dBm}$

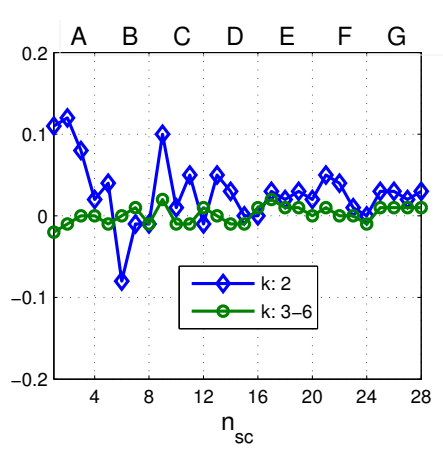

(b) $\Delta \mathrm{EVM}[\%]$

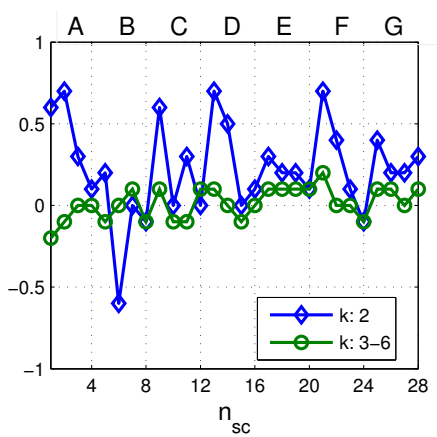

(e) $\Delta \mathrm{NMSE}_{\mathrm{IB}}[\mathrm{dB}]$

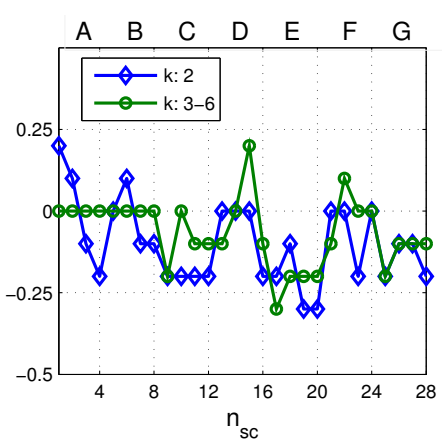

(h) $\triangle \mathrm{ACLR}[\mathrm{dB}]$

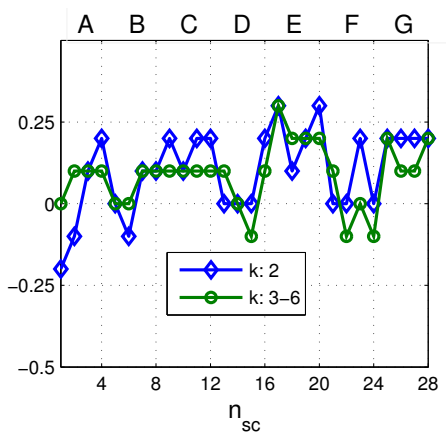

(k) $\triangle \mathrm{ACEPR}[\mathrm{dB}]$

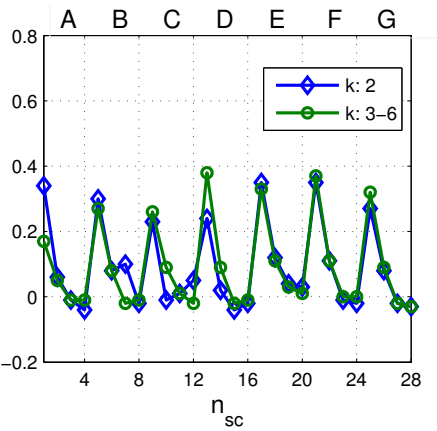

(c) $\triangle \mathrm{EVM}[\%]$

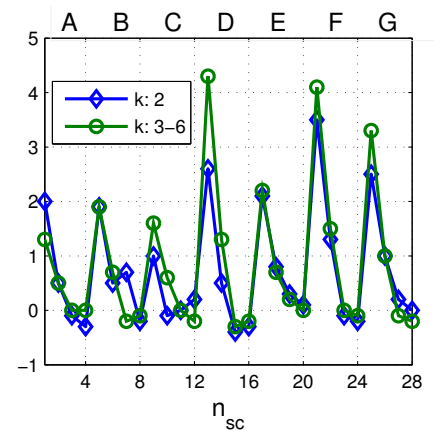

(f) $\Delta \mathrm{NMSE}_{\mathrm{IB}}[\mathrm{dB}]$

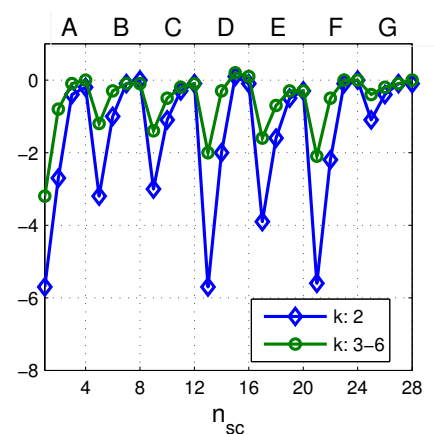

(i) $\triangle \mathrm{ACLR}[\mathrm{dB}]$

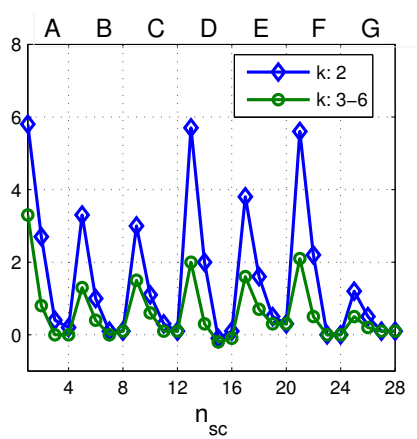

(l) $\triangle \mathrm{ACEPR}[\mathrm{dB}]$

Fig. A.1: Measurement results, $\Delta \mathrm{NMSE}_{\mathrm{IB}}, \Delta \mathrm{EVM}, \Delta \mathrm{ACLR}$ and $\triangle \mathrm{ACEPR}$ 
Frequency flat cross-talk $\mathrm{PEP}_{\text {in }}=10 \mathrm{dBm}$

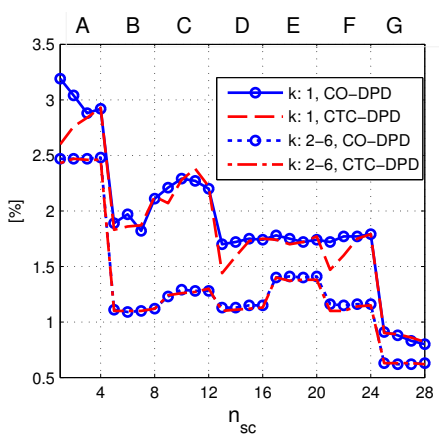

(a) EVM [\%]

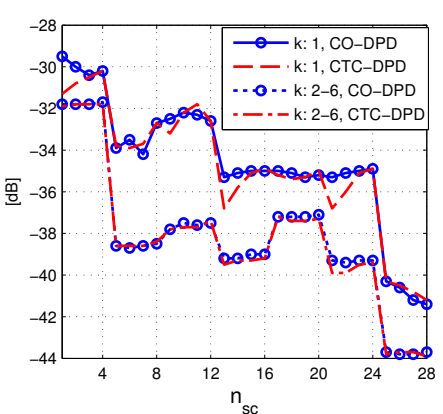

(d) $\mathrm{NMSE}_{\mathrm{IB}}[\mathrm{dB}]$

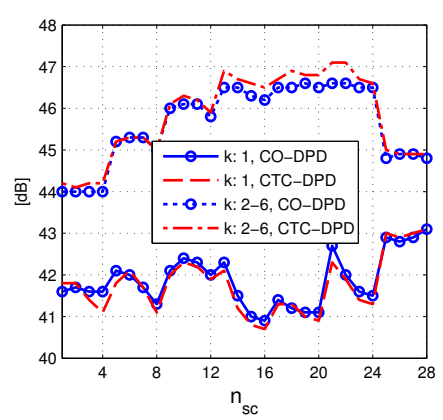

(g) ACLR $[\mathrm{dB}]$

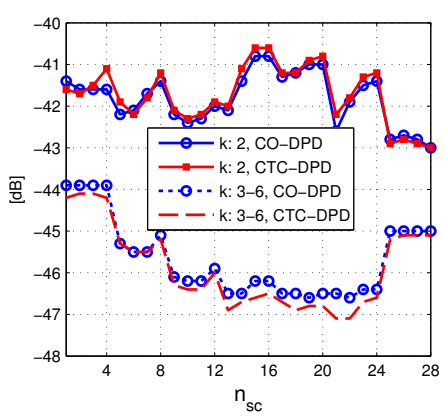

(j) ACEPR $[\mathrm{dB}]$
Frequency flat cross-talk

$\mathrm{PEP}_{\text {in }}=7 \mathrm{dBm}$

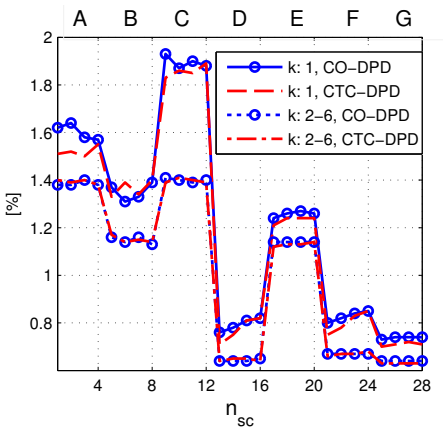

(b) $\operatorname{EVM}[\%]$

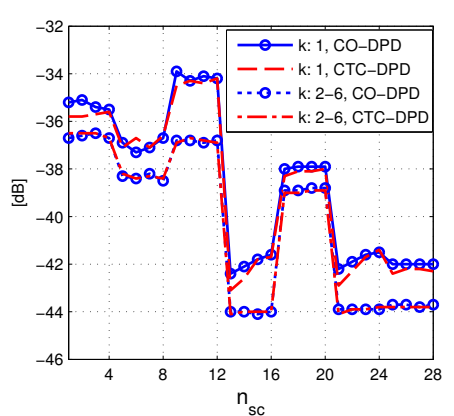

(e) $\mathrm{NMSE}_{\mathrm{IB}}[\mathrm{dB}]$

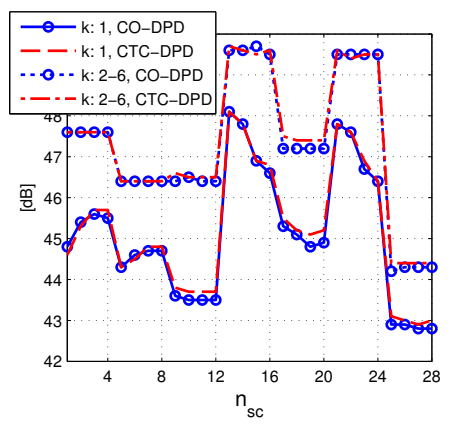

(h) ACLR $[\mathrm{dB}]$

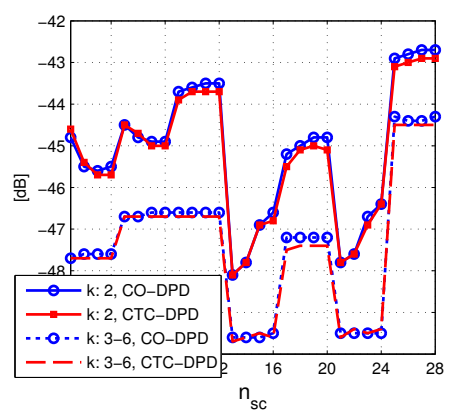

(k) ACEPR $[\mathrm{dB}]$
Frequency selective cross-talk

$\mathrm{PEP}_{\text {in }}=7 \mathrm{dBm}$

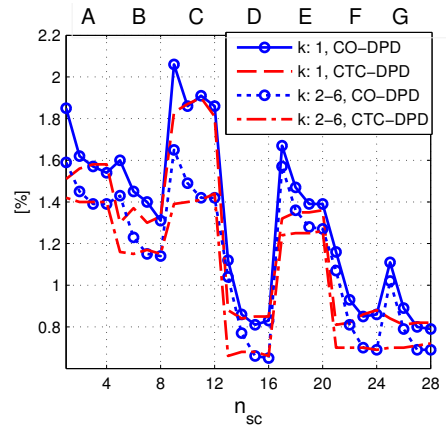

(c) $\operatorname{EVM}[\%]$

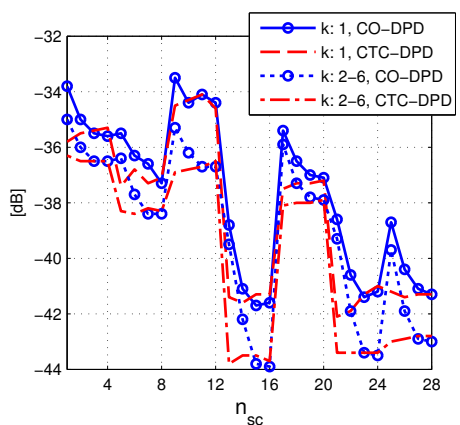

(f) $\operatorname{NMSE}_{\mathrm{IB}}[\mathrm{dB}]$

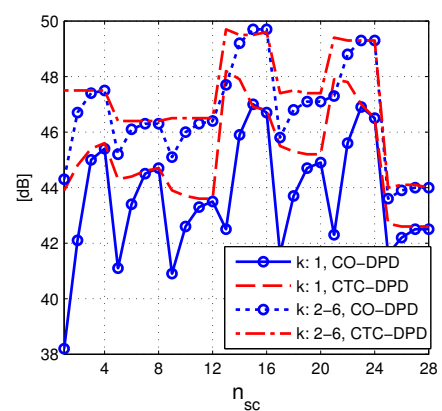

(i) ACLR $[\mathrm{dB}]$

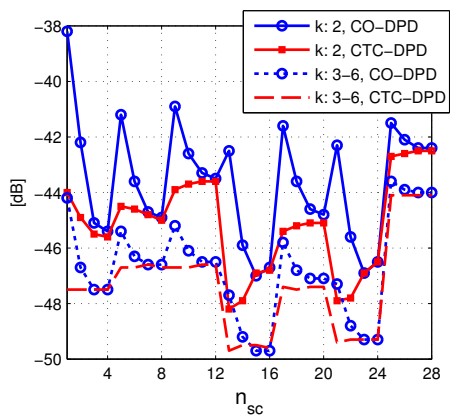

(l) ACEPR $[\mathrm{dB}]$

Fig. A.2: Measurement results, $\mathrm{NMSE}_{\mathrm{IB}}, \mathrm{EVM}, \mathrm{ACLR}$ and ACEPR 
Frequency flat

cross-talk

$\mathrm{PEP}_{\text {in }}=10 \mathrm{dBm}$

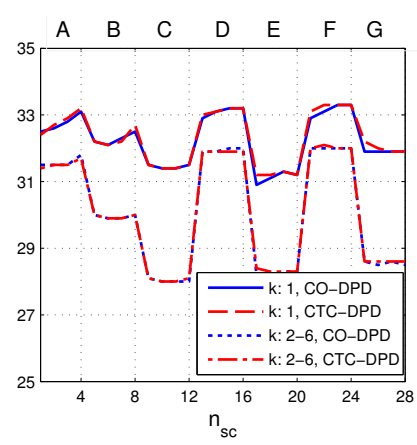

(a) $\mathrm{P}_{\text {out }}[\mathrm{dBm}]$

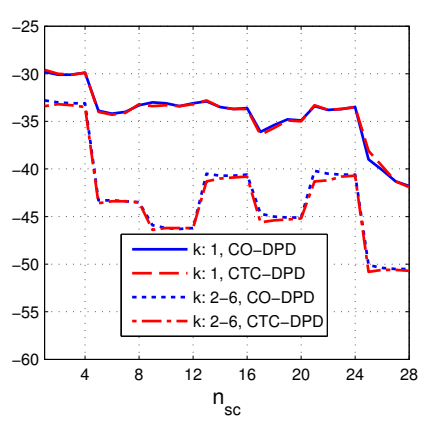

(d) $\varepsilon_{L S}[\mathrm{~dB}]$

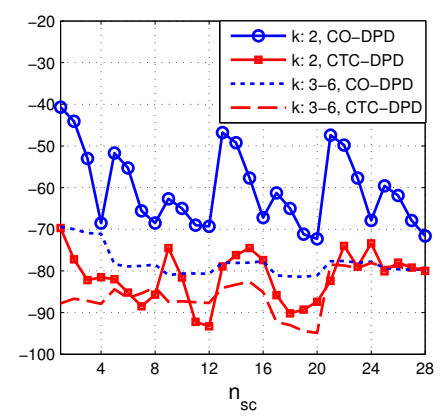

(g) $\varepsilon_{\text {Leak }}[\mathrm{dB}]$

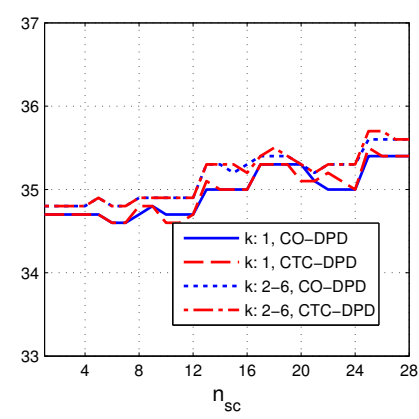

(j) $\mathrm{PEP}_{\text {out }}[\mathrm{dBm}]$
Frequency flat cross-talk

$\mathrm{PEP}_{\text {in }}=7 \mathrm{dBm}$

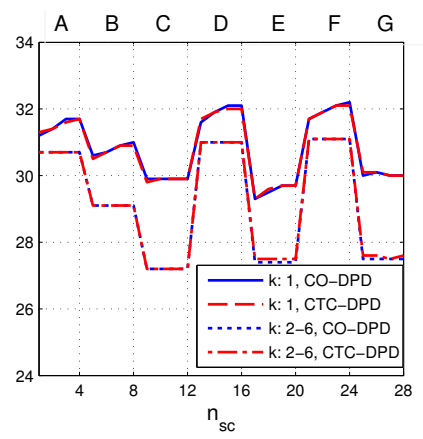

(b) $\mathrm{P}_{\text {out }}[\mathrm{dBm}]$

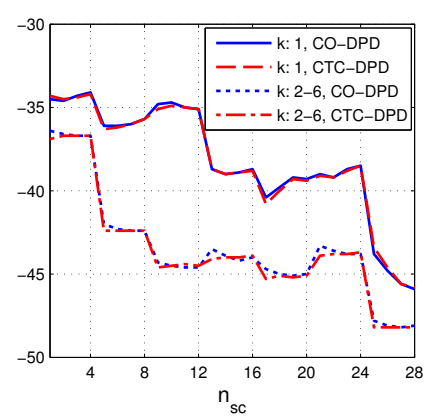

(e) $\varepsilon_{L S}[\mathrm{~dB}]$

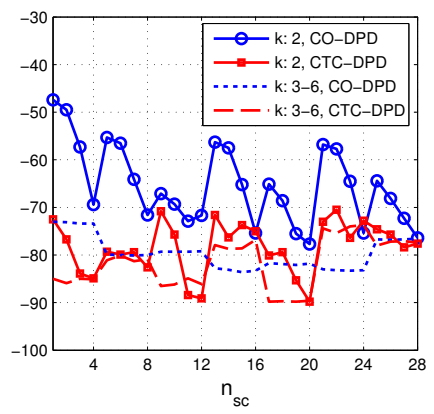

(h) $\varepsilon_{\text {Leak }}[\mathrm{dB}]$

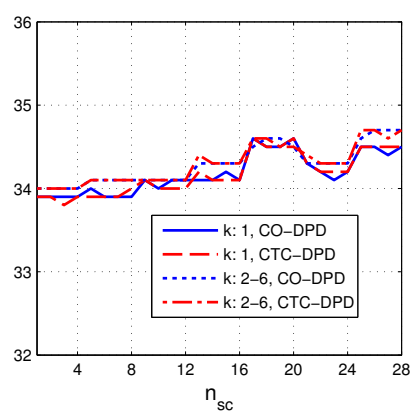

(k) $\mathrm{PEP}$ out $[\mathrm{dBm}]$
Frequency selective

cross-talk

$\mathrm{PEP}_{\text {in }}=7 \mathrm{dBm}$

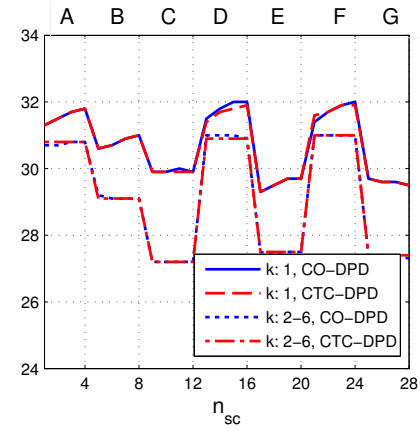

(c) $P_{\text {out }}[\mathrm{dBm}]$

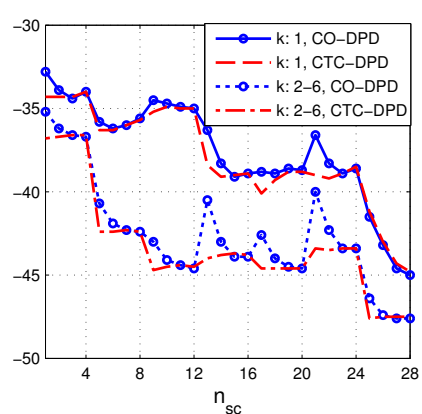

(f) $\varepsilon_{L S}[\mathrm{~dB}]$

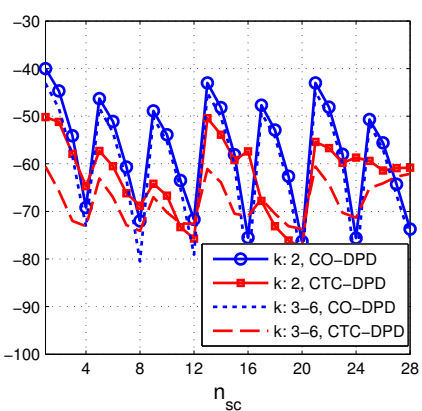

(i) $\varepsilon_{\text {Leak }}[\mathrm{dB}]$

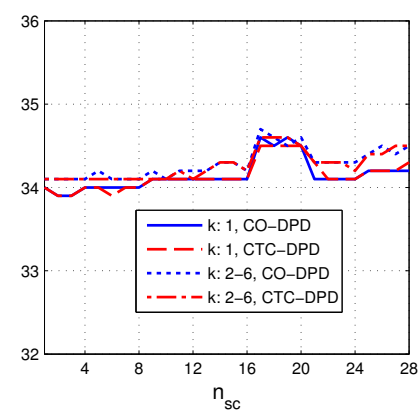

(l) $\mathrm{PEP}_{\text {out }}[\mathrm{dBm}]$

Fig. A.3: Measurement results, $\varepsilon_{L S}, \varepsilon_{\text {Leak }}, \mathrm{P}_{\text {out }}$ and $\mathrm{PEP}_{\text {out }}$ 


\section{Appendix B.}

\section{Measurement results (Single antenna Tx)}

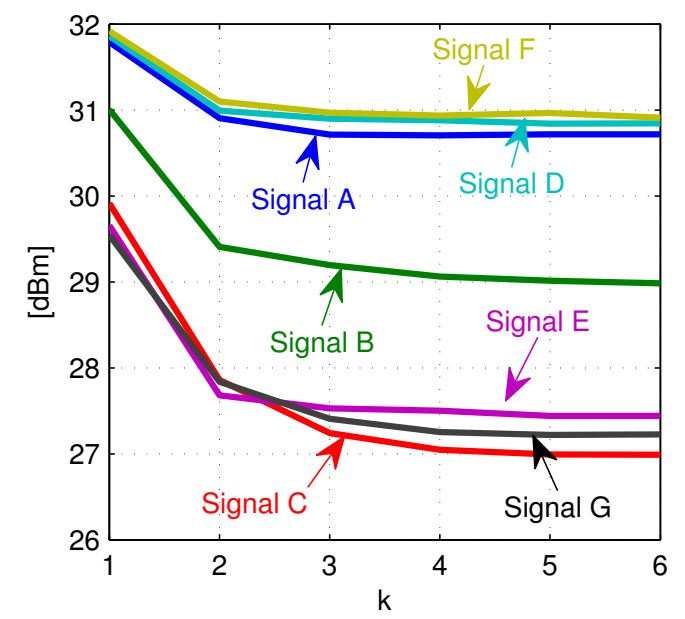

(a) $\mathrm{PEP}_{\text {in }}=7 \mathrm{dBm}$

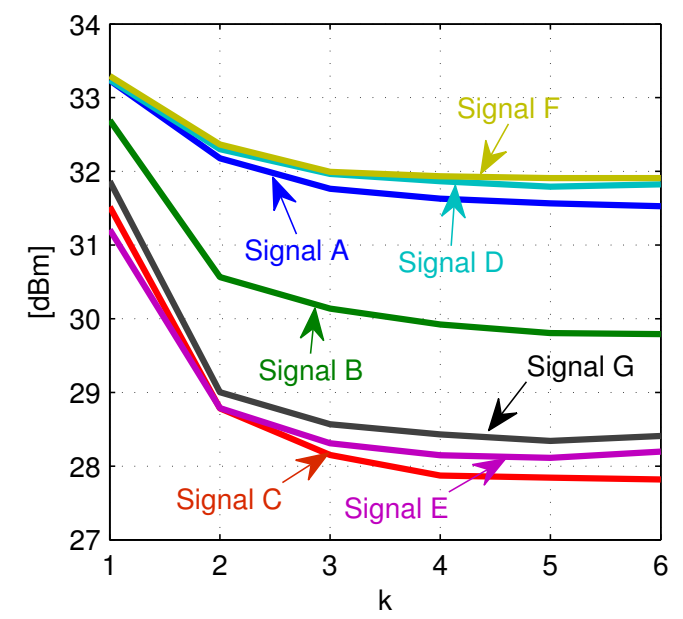

(b) $\mathrm{PEP}_{\text {in }}=10 \mathrm{dBm}$

Fig. B.1: PA output power

In this chapter, we present the measurement results (PA output power, AM-AM and AM-PM characteristics, constellation diagrams, PSD spectra, etc.) for a single antenna transmitter, which are the same as that of a multiple antenna transmitter without cross-talk. These are very important indicators of the PA behavior, but they are not used in the comparison of the linearization performances of C-DPD, CO-DPD and CTC-DPD in chapters 3 and 4 . That is why we present them in this chapter.

Fig. B.1 shows the PA output power for different transmit signals for the two PA responses which we have chosen for our measurements. The details of these signals are given in Table 3.2. The two PA responses correspond to the measurements done by keeping the peak envelope power of the input signal of the PA $\left(\mathrm{PEP}_{\text {in }}\right)$ at $7 \mathrm{dBm}$ and $10 \mathrm{dBm}$. The predistortion is carried out in a block-by-block manner as 
Table B.1.: PA output power $[\mathrm{dBm}]$

\begin{tabular}{|c|c|c|c|c|c|c|c|c|c|}
\hline Signal $\rightarrow$ & A & B & $\mathrm{C}$ & $\mathrm{D}$ & $\mathrm{E}$ & $\mathrm{F}$ & G & $\mathrm{DPD}$ & Power back-off \\
\hline \multirow{2}{*}{ Set 1} & 30.7 & 29 & 27 & 30.8 & 27.4 & 30.9 & 27.2 & No & Yes \\
\hline & \multicolumn{7}{|c|}{ As shown in Fig. B.1a } & Yes & $\mathrm{No}$ \\
\hline \multirow{2}{*}{ Set 2} & 31.5 & 29.8 & 27.8 & 31.8 & 28.1 & 31.9 & 28.4 & No & Yes \\
\hline & \multicolumn{7}{|c|}{ As shown in Fig. B.1b } & Yes & $\mathrm{No}$ \\
\hline
\end{tabular}

described in chapter 3. The index $k$, which is used in all the figures in this chapter denote the block number. Predistorter is applied for the blocks $k=2,3, . .6$, but not for the first block $(k=1)$.

The AM-AM and AM-PM characteristics and PSD spectra are shown in Fig. B.1Fig. B.4. We define two quantities $\varepsilon_{\text {amp }}$ and $\varepsilon_{\text {arg }}$ to quantify the distortion introduced by AM-AM and AM-PM conversions from the PA.

$$
\begin{aligned}
\varepsilon_{\mathrm{amp}} & =\frac{1}{N} \sum_{n=1}^{N}(|y(n)|-|x(n)|)^{2} \\
\varepsilon_{\mathrm{arg}} & =\frac{1}{N} \sum_{n=1}^{N}(\arg (y(n))-\arg (x(n)))^{2}
\end{aligned}
$$

where $x(n)$ and $y(n)$ are the PA input and output signals respectively. Fig. B.5 and Fig. B.6 show the behavior of $\varepsilon_{\text {amp }}$ and $\varepsilon_{\text {arg }}$ for different cases. The constellation diagrams for signal B (QPSK) and signal C (16-QAM) with and without the application of DPD, are illustrated in Fig. B.7 and Fig. B.8. Fig. B.1-Fig. B.8 show that predistortion brings forth a considerable improvement in the linearization performance, and some reduction in the output power with our measurement settings.

The reduction in the output power due to predistortion-which can be seen in Fig. B.1 - raises a question: Reducing the PA output power improves the PA linearity, so, is predistortion better than simply backing off the output power? In general, this depends on the PA operating condition [60]. To make sure that DPD brings an improvement in linearization for the PA responses compared to a power back-off, we compare EVM and ACLR of these two cases (i.e., DPD with no power back-off and no-DPD with power back-off). The backed off power levels are given in Table B.1. We have chosen the power back-off equal to the power reduction caused by DPD, so that the backed off power levels are the same as in Fig. B.1 at $k=6$. We carried out 2 sets of these measurements for the two PA responses used in our measurements. The EVM and ACLR for these cases are shown in Fig. B.9 and Fig. B.10. It can be seen, that predistortion achieves a better linearization performance, compared to power back-off for all the signals considered here. 


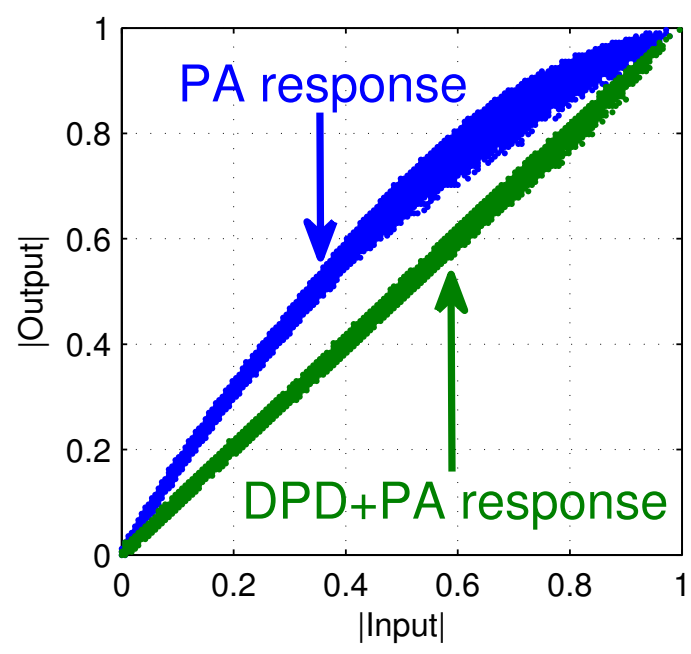

(a) $\mathrm{PEP}_{\text {in }}=7 \mathrm{dBm}$

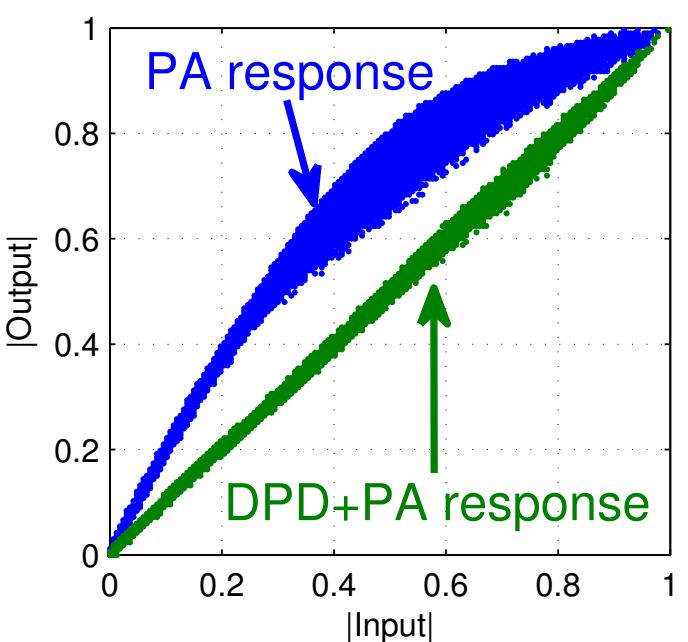

(b) $\mathrm{PEP}_{\text {in }}=10 \mathrm{dBm}$

Fig. B.2: AM-AM plots (signal E), $k=1,6$

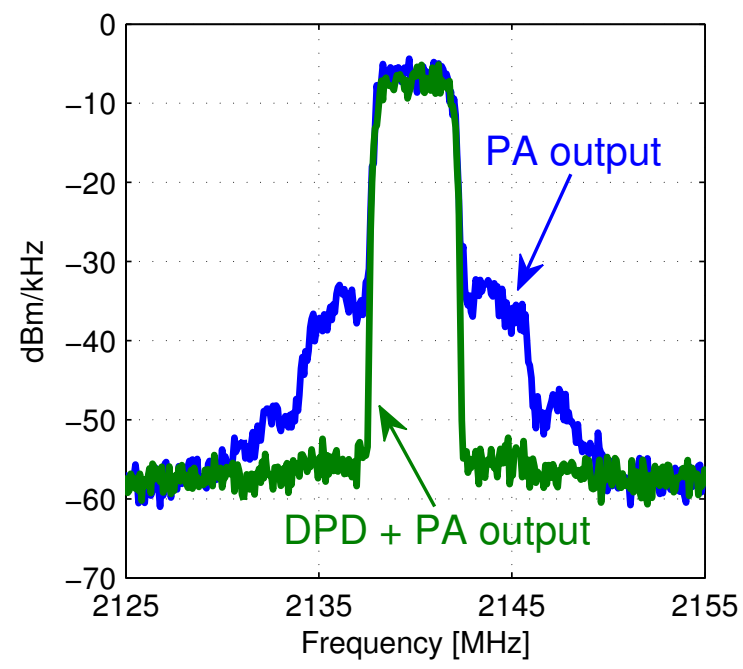

(a) $\mathrm{PEP}_{\text {in }}=7 \mathrm{dBm}$

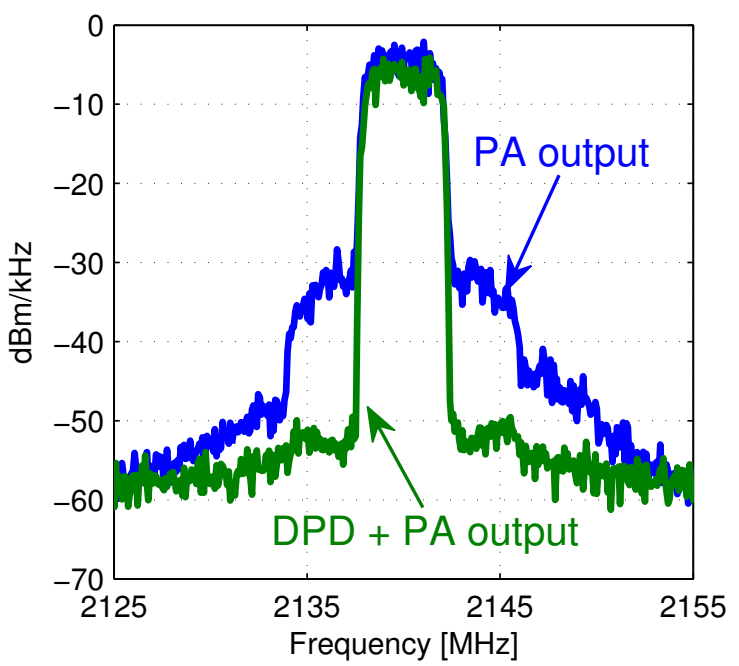

(b) $\mathrm{PEP}_{\text {in }}=10 \mathrm{dBm}$

Fig. B.3: PSD spectra (signal E), $k=1,6$ 


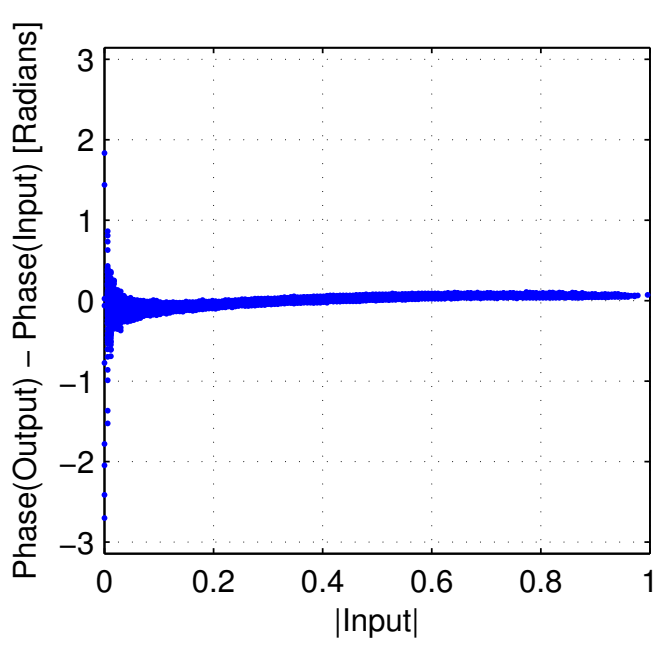

(a) $\mathrm{PEP}_{\text {in }}=7 \mathrm{dBm}, k=1$

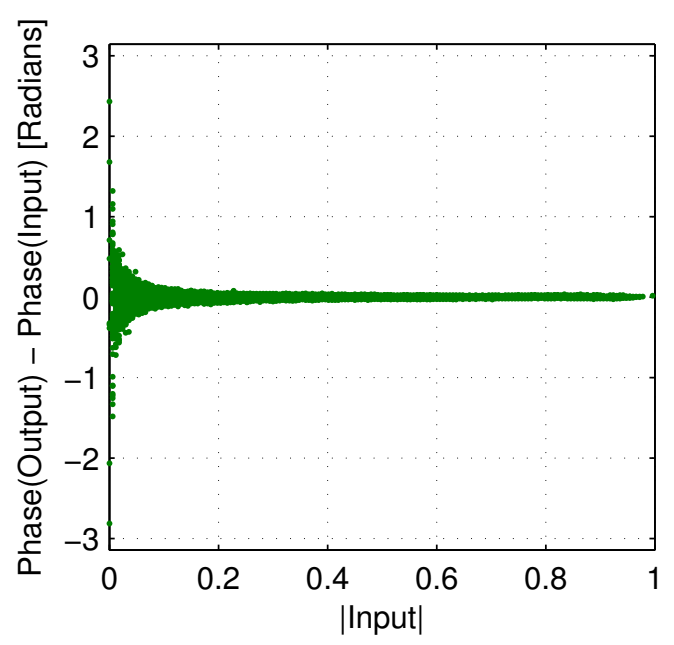

(c) $\mathrm{PEP}_{\text {in }}=7 \mathrm{dBm}, k=6$

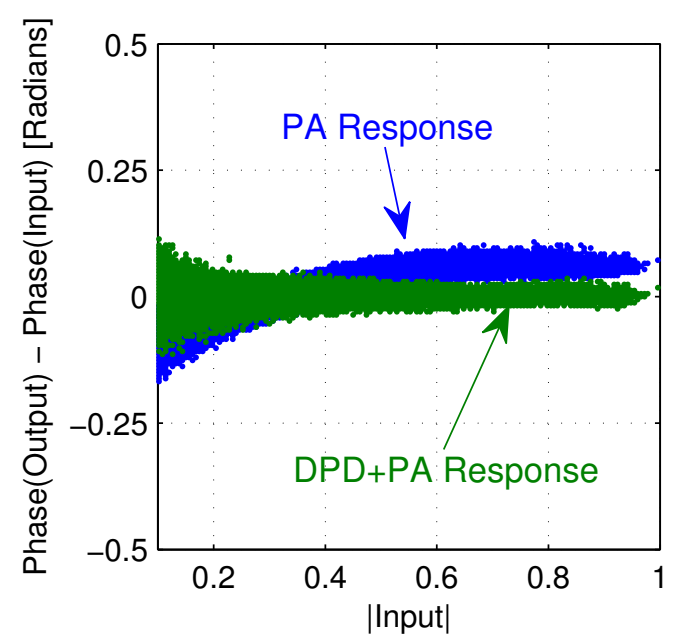

(e) $\mathrm{PEP}_{\text {in }}=7 \mathrm{dBm}, k=1,6$,

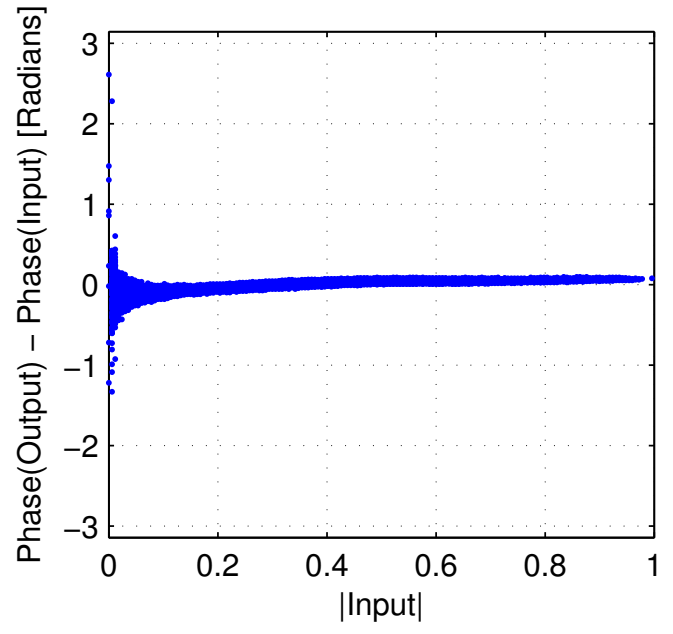

(b) $\mathrm{PEP}_{\text {in }}=10 \mathrm{dBm}, k=1$

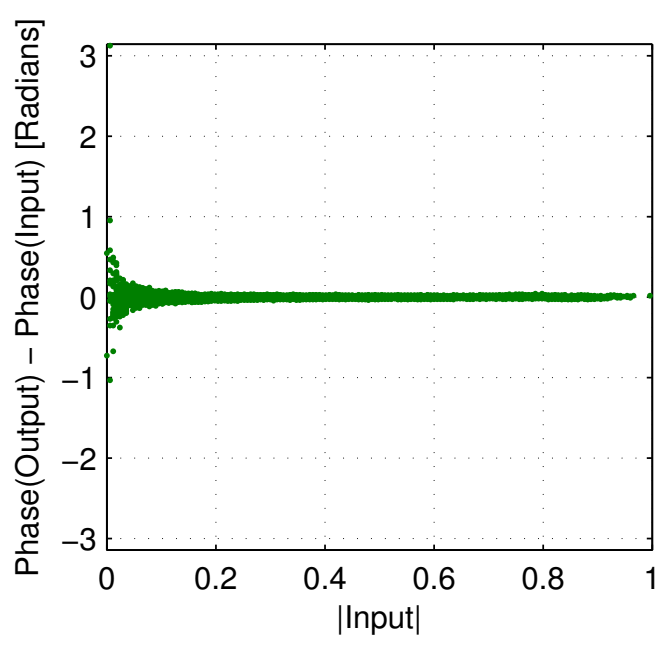

(d) $\mathrm{PEP}_{\text {in }}=10 \mathrm{dBm}, k=6$

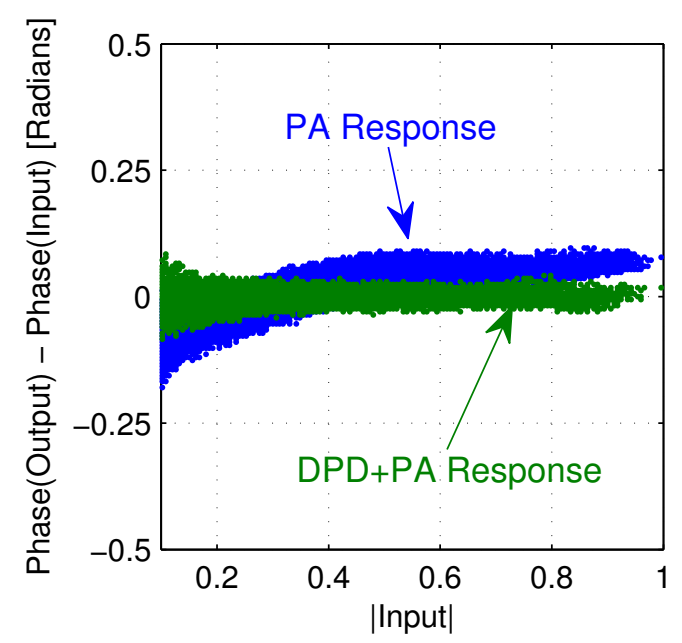

(f) $\mathrm{PEP}_{\text {in }}=10 \mathrm{dBm}, k=1,6$

Fig. B.4: AM-PM plots (signal E) 


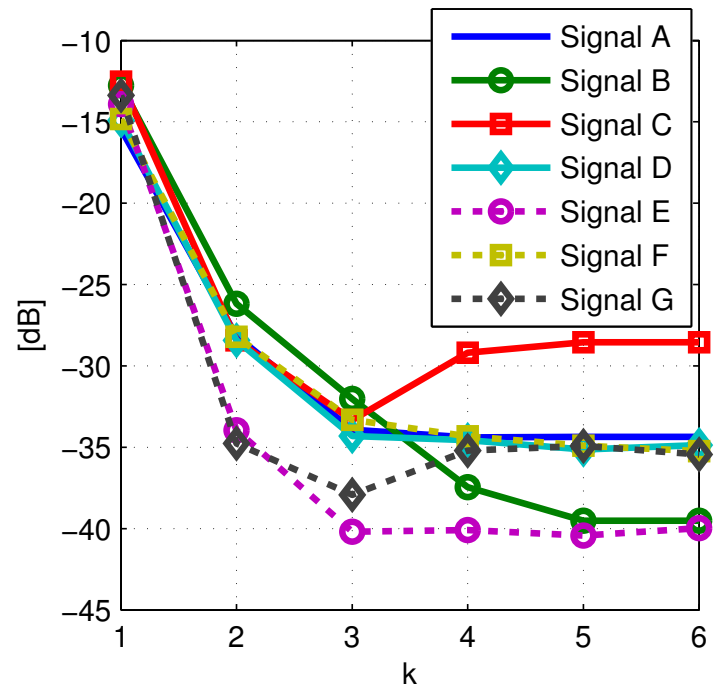

(a) $\mathrm{PEP}_{\text {in }}=7 \mathrm{dBm}$

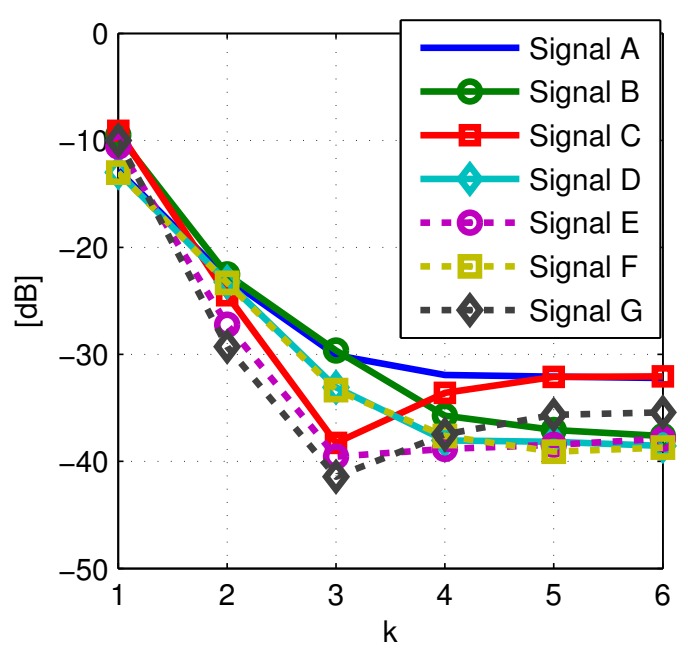

(b) $\mathrm{PEP}_{\text {in }}=10 \mathrm{dBm}$

Fig. B.5: $\varepsilon_{\text {amp }}$

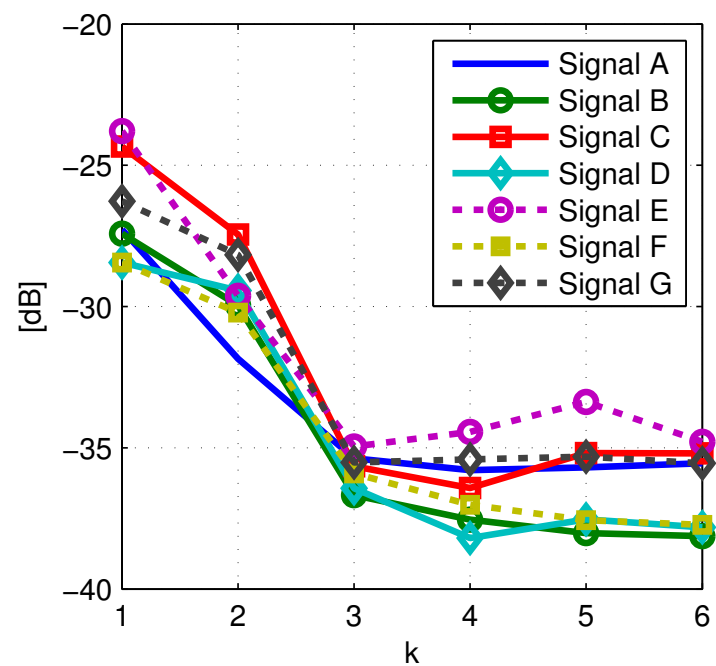

(a) $\mathrm{PEP}_{\text {in }}=7 \mathrm{dBm}$

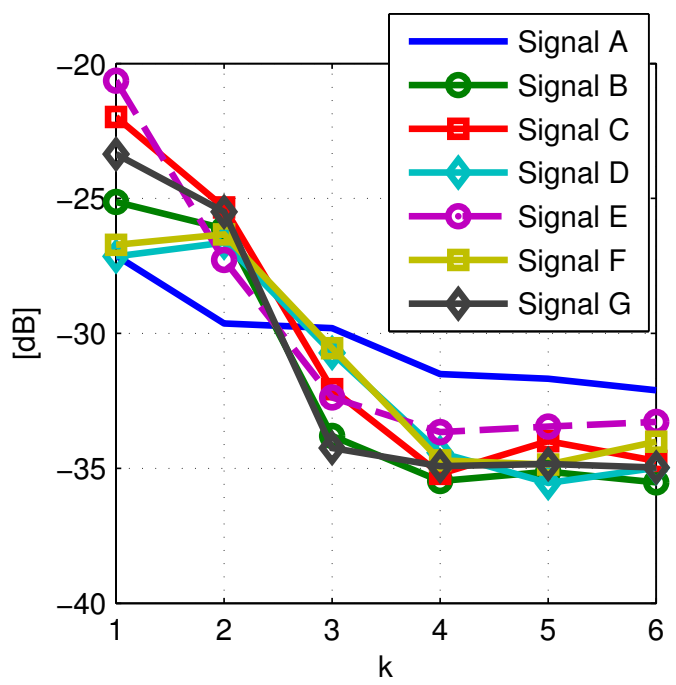

(b) $\mathrm{PEP}_{\text {in }}=10 \mathrm{dBm}$

Fig. B.6: $\varepsilon_{\text {arg }}$ 


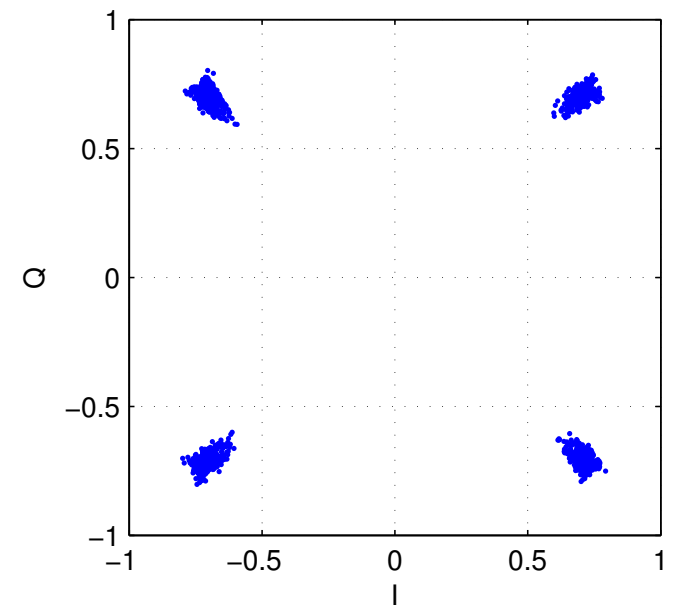

(a) Signal B, no DPD, $k=1$

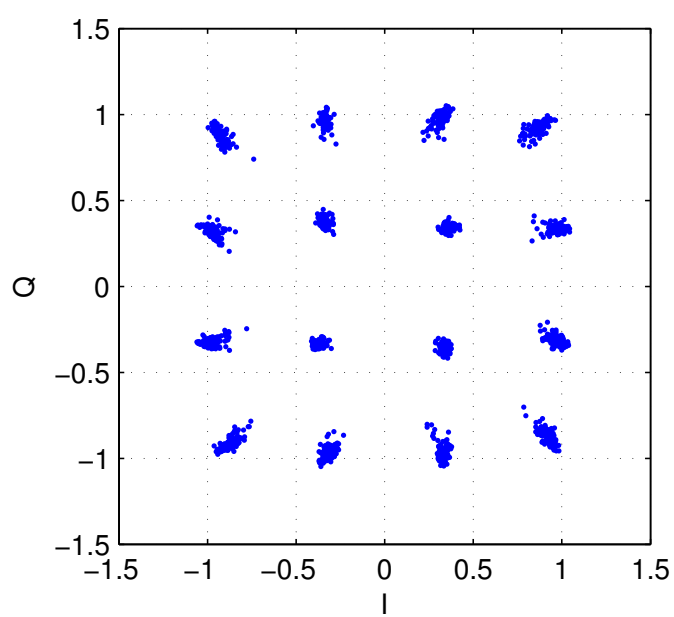

(c) Signal C, no DPD, $k=1$

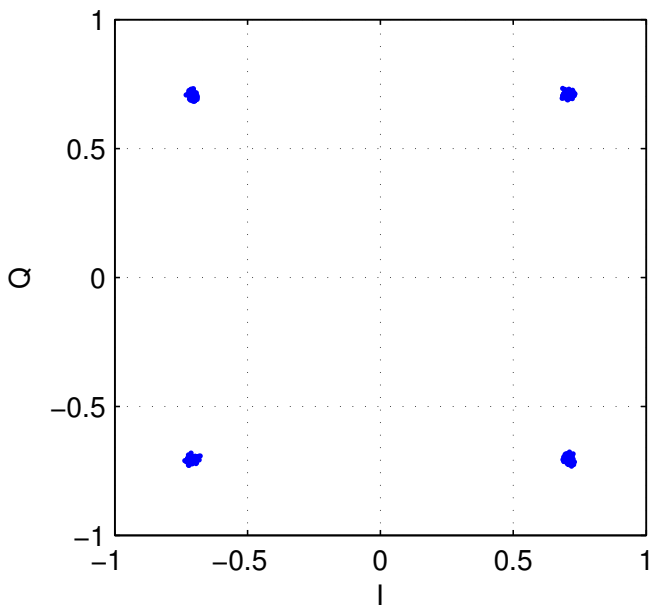

(b) Signal B, with DPD, $k=6$

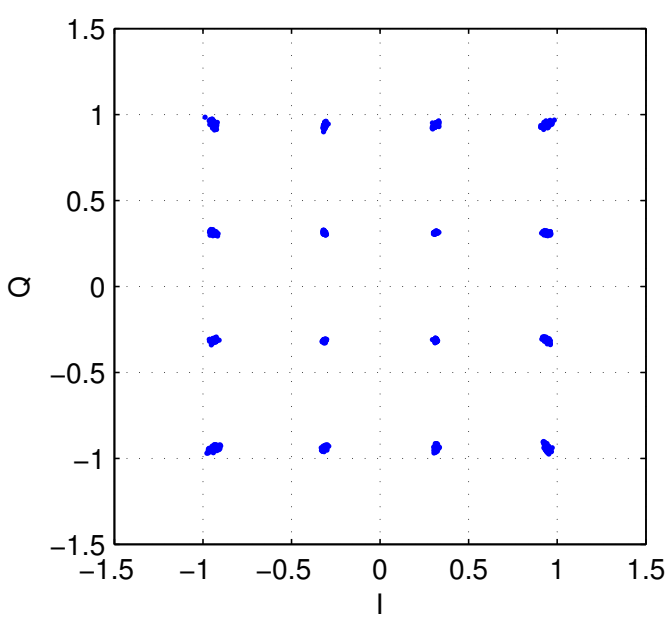

(d) Signal B, with DPD, $k=6$

Fig. B.7: Constellation diagrams, $\mathrm{PEP}_{\mathrm{in}}=7 \mathrm{dBm}$ 


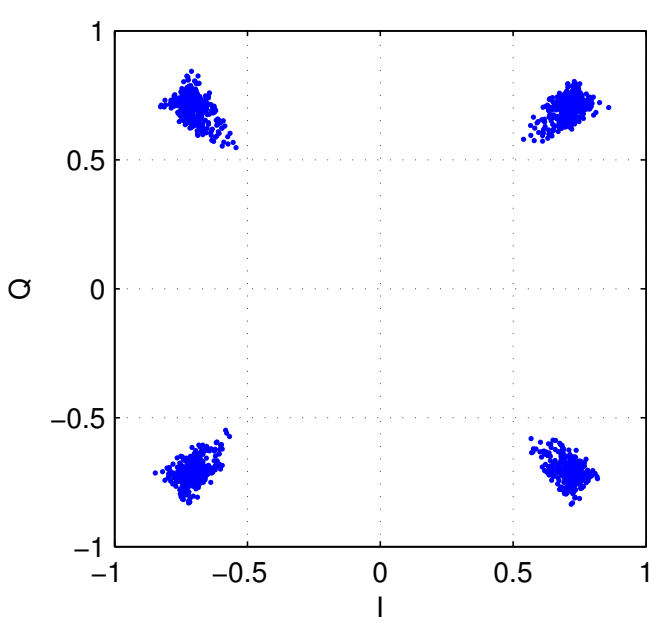

(a) Signal B, no DPD, $k=1$

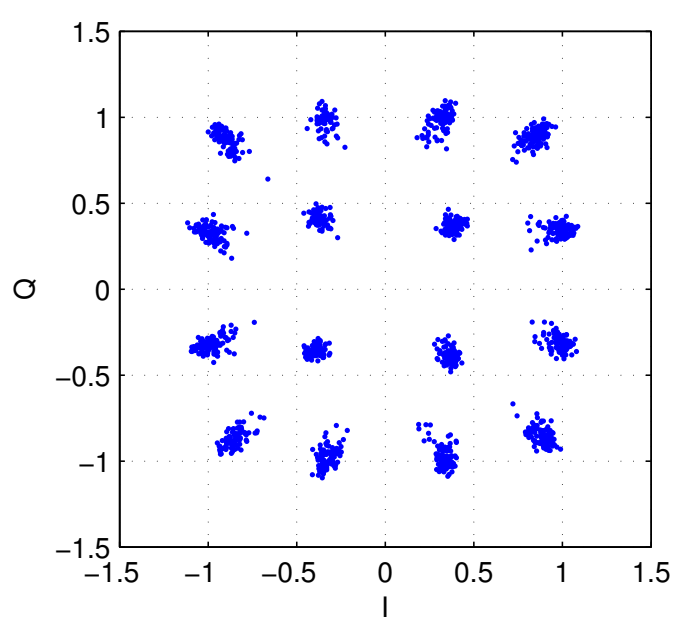

(c) Signal C, no DPD, $k=1$

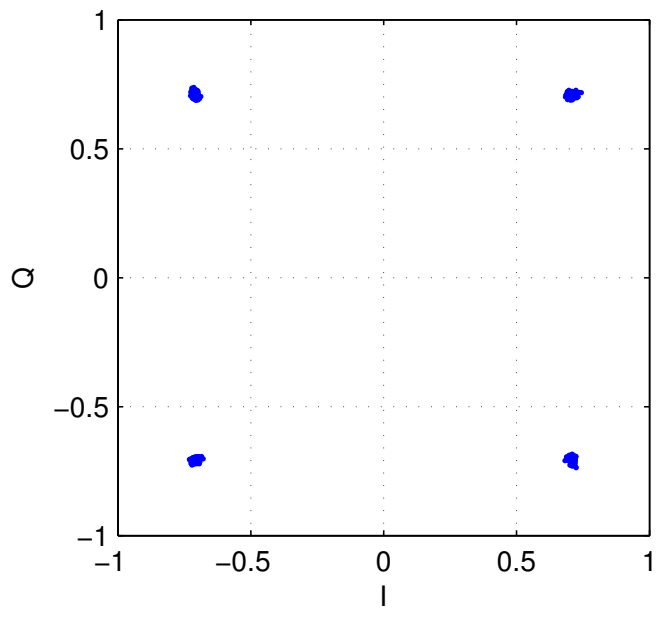

(b) Signal B, with DPD, $k=6$

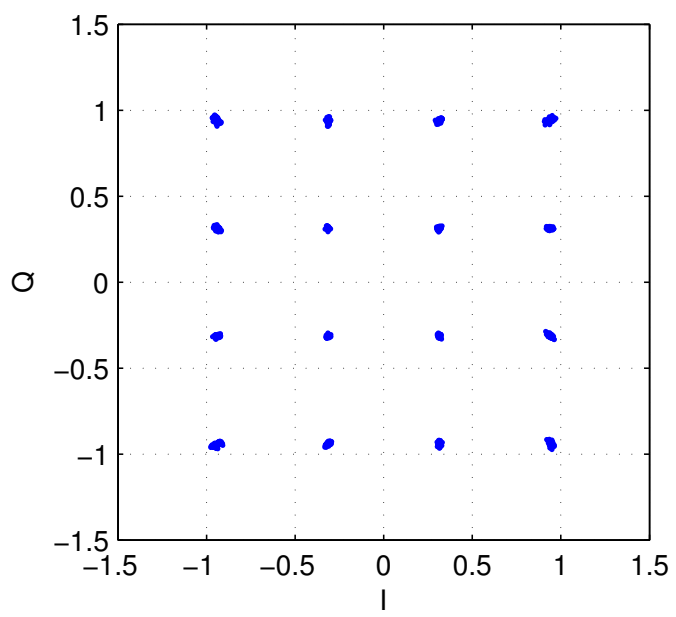

(d) Signal C, with DPD, $k=6$

Fig. B.8: Constellation diagrams, $\mathrm{PEP}_{\text {in }}=10 \mathrm{dBm}$ 


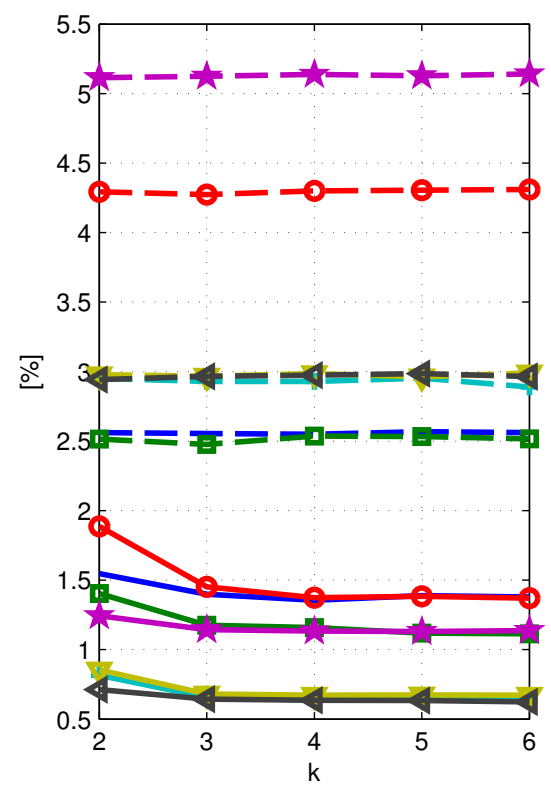

(a) EVM

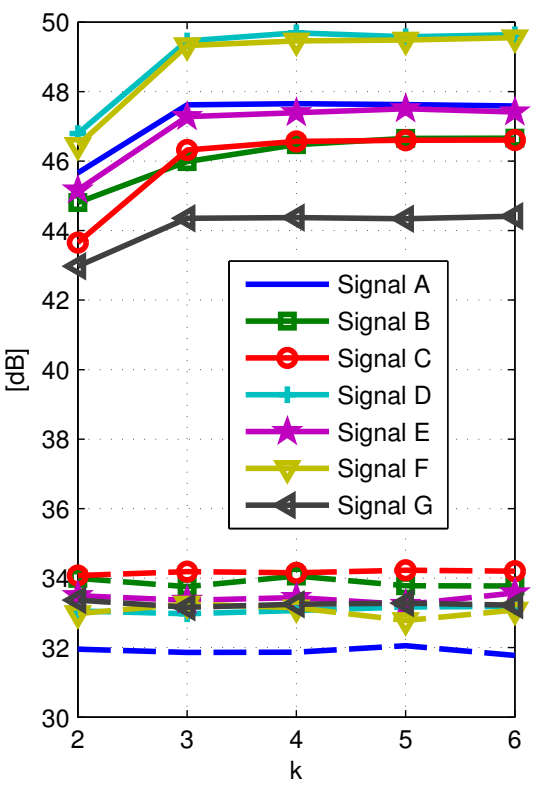

(b) ACLR

- - - No DPD, Power back-off

Fig. B.9: Set 1: EVM \& ACLR

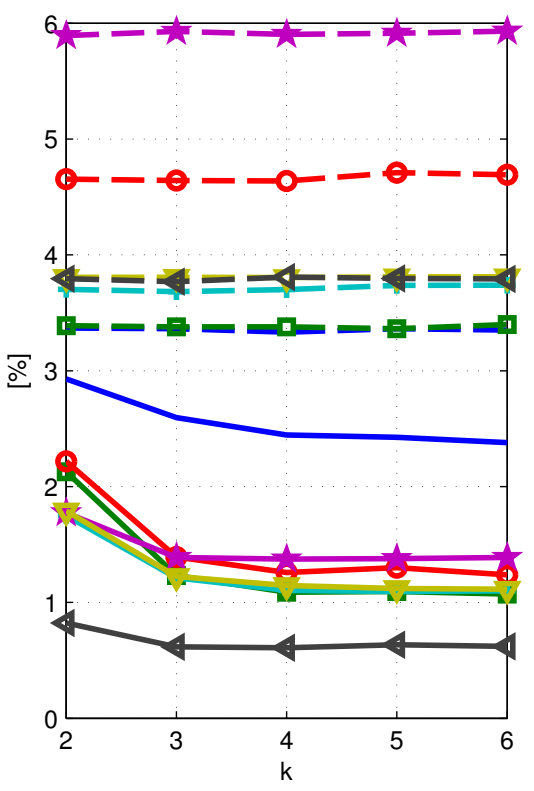

(a) EVM

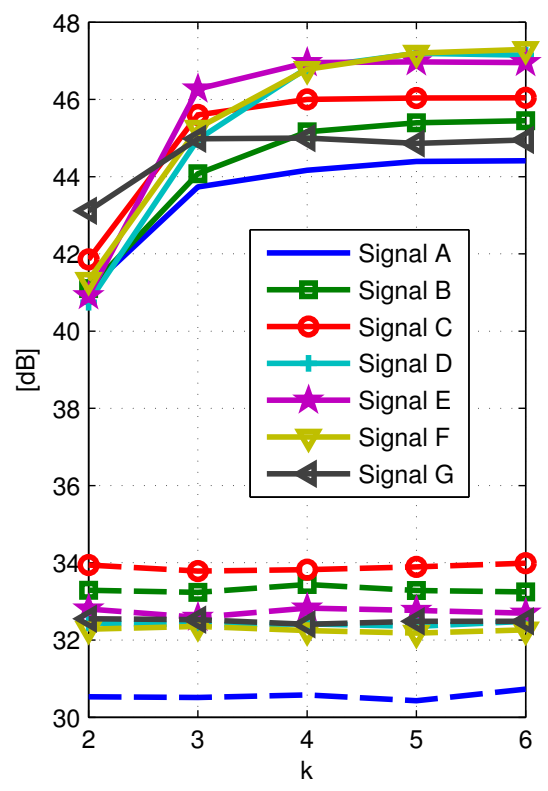

(b) ACLR

- - No DPD, Power back-off

Fig. B.10: Set 2: EVM \& ACLR 


\section{Appendix C.}

\section{Accuracy of cross-talk estimation}

In this section, we discuss the accuracy of the estimation of the coupling factors that model the cross-talk, for CTC-DPD. We define the average error in cross-talk estimation, $\varepsilon_{\alpha}$ for a two antenna transmitter as

$$
\varepsilon_{\alpha}=10 \log \left(\sum_{i=1}^{2}\left\|\hat{\boldsymbol{\alpha}}_{i}-\boldsymbol{\alpha}_{i}\right\|^{2}\right) .
$$

$\boldsymbol{\alpha}_{i}$ is the coupling filter coefficient vector of size $M \times 1$, where $M$ is the number of filter coefficients used to model the cross-talk. $\varepsilon_{\alpha}$ is evaluated for 6 data-blocks of our measurements, which are described in chapter 3. Fig. C.1 shows $\varepsilon_{\alpha}$ for different cases, for signal G. It is found that $\varepsilon_{\alpha}$ is smaller (i.e, better cross-talk estimation) for all other signals compared to signal G. Fig. C.2 shows $\varepsilon_{\alpha}$ (for signal G) for the adaptive MIMO-DPD algorithms with LMS cross-talk estimation, described in chapter 4 . It can be seen that the cross-talk estimation error is below $-62 \mathrm{~dB}$ for frequency flat cross-talk and below $-44 \mathrm{~dB}$ for frequency selective ${ }^{1}$ cross-talk, for block based operation. In the case of sample-by-sample operation, the cross-talk estimation error converges at around $-55 \mathrm{~dB}$ for frequency flat cross-talk and -45 $\mathrm{dB}$ for frequency selective cross-talk.

As the cross-talk coefficients are estimated from the linear section of the PA response, the memory present in the linear section of the PA will degrade the quality of crosstalk estimates. To understand this, we carry out simulations using the PA input signals and artificially introducing memory effects. According to (3.23), the vectors of complex envelopes of the input signals to the PAs are

$$
\begin{aligned}
& \boldsymbol{u}_{1}=\left[\begin{array}{ll}
\boldsymbol{x}_{1} & \boldsymbol{B}_{x_{2}}
\end{array}\right]\left[\begin{array}{c}
1 \\
\boldsymbol{\alpha}_{1}
\end{array}\right] \\
& \boldsymbol{u}_{2}=\left[\begin{array}{ll}
\boldsymbol{x}_{2} & \boldsymbol{B}_{x_{1}}
\end{array}\right]\left[\begin{array}{c}
1 \\
\boldsymbol{\alpha}_{2}
\end{array}\right],
\end{aligned}
$$

(C.2) represents frequency flat $(M=1)$ and frequency selective $(M>1)$ crosstalk. In the case of frequency flat cross-talk, $\boldsymbol{B}_{x_{1}}$ and $\boldsymbol{B}_{x_{2}}$ reduces to $\boldsymbol{x}_{1}$ and $\boldsymbol{x}_{2}$

\footnotetext{
${ }^{1}$ Filter 1a, as shown in Fig. 3.19, is used to model the frequency selective cross-talk, in all the results presented in this chapter.
} 
Frequency flat

cross-talk

$\mathrm{PEP}_{\text {in }}=10 \mathrm{dBm}$
Frequency flat

cross-talk

$\mathrm{PEP}_{\text {in }}=7 \mathrm{dBm}$
Frequency selective

cross-talk

$\mathrm{PEP}_{\text {in }}=7 \mathrm{dBm}$

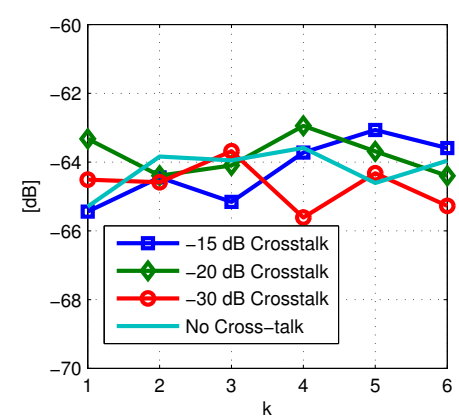

(a)

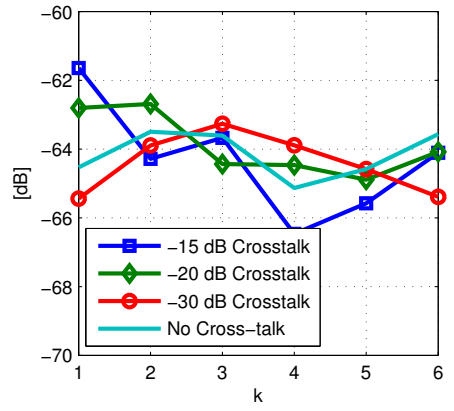

(b)

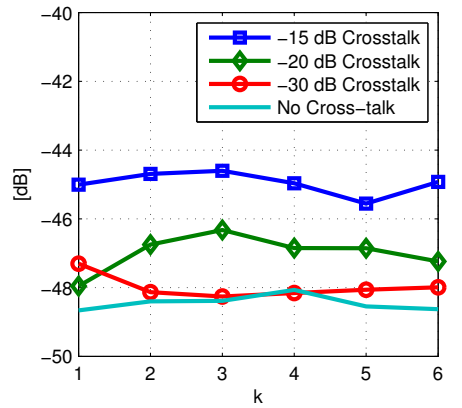

(c)

Fig. C.1: Average error in cross-talk estimation $\left(\varepsilon_{\alpha}\right)$ in block based cross-talk estimation, signal $\mathrm{G}, k$ is the block number.

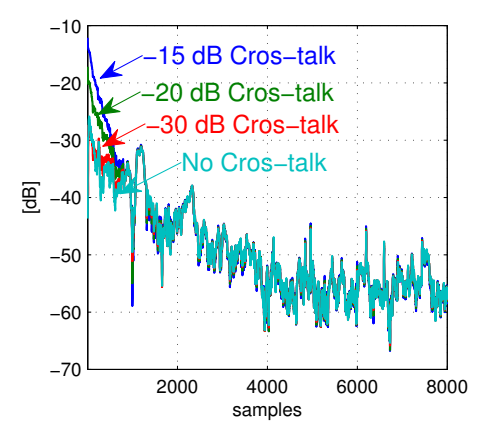

(a)

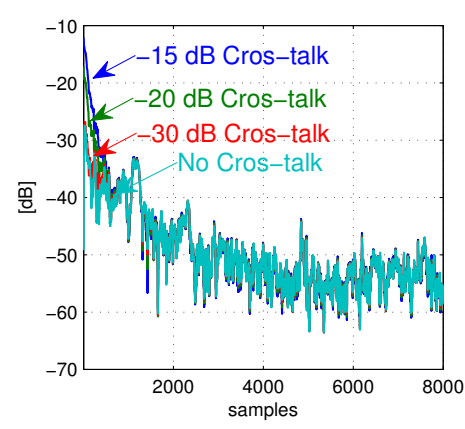

(b)

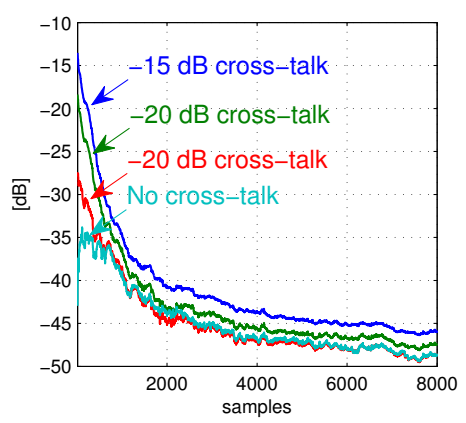

(c)

Fig. C.2: Average error in cross-talk estimation $\left(\varepsilon_{\alpha}\right)$ in sample-by-sample cross-talk estimation (LMS), signal G

respectively, and $\boldsymbol{\alpha}_{1}$ and $\boldsymbol{\alpha}_{2}$ reduces to the scalar values that represent coupling coefficients, $\alpha_{1}$ and $\alpha_{2}$. If $\boldsymbol{u}_{1}$ and $\boldsymbol{u}_{2}$ are available, $\boldsymbol{\alpha}_{1}$ and $\boldsymbol{\alpha}_{2}$ can be estimated perfectly from pinv $\left(\left[\begin{array}{ll}\boldsymbol{x}_{1} & \boldsymbol{B}_{x_{2}}\end{array}\right]\right) \boldsymbol{u}_{1}$ and pinv $\left(\left[\boldsymbol{x}_{2} \boldsymbol{B}_{x_{1}}\right]\right) \boldsymbol{u}_{2}$ respectively. But, accessing $u_{1}(n)$ and $u_{2}(n)$ may not be practically feasible, so we use the linear section of $y_{1}$ and $y_{2}$ for the cross-talk estimation in the measurements, as discussed in section 3.2. Now, we add memory terms to (C.2).

$$
\begin{aligned}
& \boldsymbol{u}_{1}^{\prime}=\left[\begin{array}{ll}
\boldsymbol{x}_{1} & \boldsymbol{B}_{x_{2}}
\end{array}\right]\left[\begin{array}{c}
1 \\
\boldsymbol{\alpha}_{1}
\end{array}\right]+\sum_{d=1}^{D} m_{1}(d)\left[\begin{array}{ll}
\boldsymbol{x}_{1, d} & \boldsymbol{B}_{x_{2, d}}
\end{array}\right]\left[\begin{array}{c}
1 \\
\boldsymbol{\alpha}_{1}
\end{array}\right] \\
& \boldsymbol{u}_{2}^{\prime}=\left[\begin{array}{ll}
\boldsymbol{x}_{2} & \boldsymbol{B}_{x_{1}}
\end{array}\right]\left[\begin{array}{c}
1 \\
\boldsymbol{\alpha}_{2}
\end{array}\right]+\sum_{d=1}^{D} m_{2}(d)\left[\begin{array}{ll}
\boldsymbol{x}_{2, d} & \boldsymbol{B}_{x_{1, d}}
\end{array}\right]\left[\begin{array}{c}
1 \\
\boldsymbol{\alpha}_{2}
\end{array}\right],
\end{aligned}
$$

where $\boldsymbol{x}_{1, d}$ and $\boldsymbol{x}_{2, d}$ are the signal vectors containing the samples of $x_{1, d}(n)$ and 


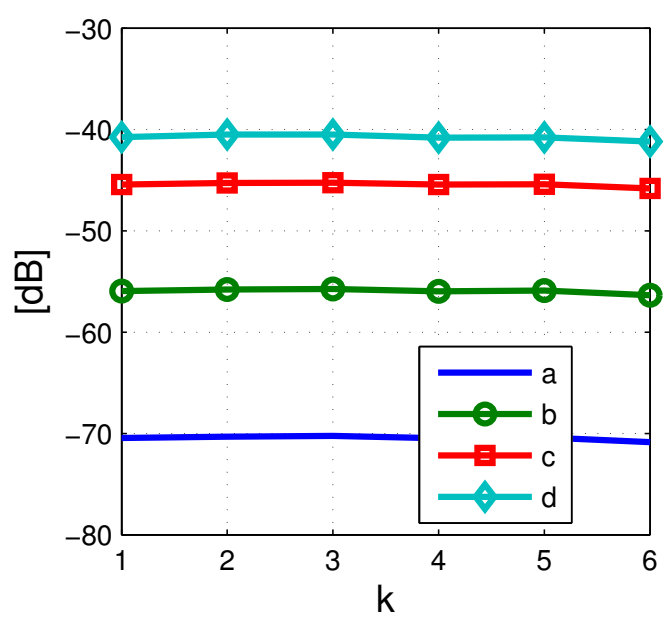

(a) Frequency flat cross-talk

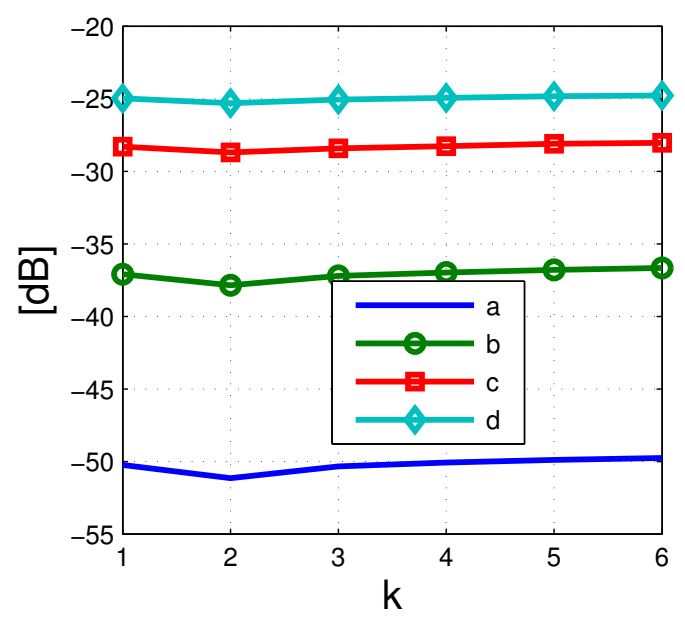

(b) Frequency selective cross-talk

Fig. C.3: Average error in cross-talk estimation $\left(\varepsilon_{\alpha}\right)$ from the simulations as described in (C.4), signal G, $\mathrm{PEP}_{\text {in }}=7 \mathrm{dBm},-20 \mathrm{~dB}$ cross-talk, $k$ is the block number.

$x_{2, d}(n)$ respectively, where $x_{1, d}(n)=x_{1}(n-d)$ and $x_{2, d}(n)=x_{2}(n-d) . \boldsymbol{B}_{x_{1, d}}$ and $\boldsymbol{B}_{x_{2, d}}$ are constructed similar to (3.24). $m_{1}$ and $m_{2}$ are the relative weights of the memory elements with respect to the input signal. We assume that (C.3) will closely resemble the input output relation of the PA linear section, with the memory effect. In (3.18) and (3.26), these memory terms are represented as noise. Now we carry out an analysis to see how our cross-talk estimation method works with $\boldsymbol{u}_{1}^{\prime}$ and $\boldsymbol{u}_{2}^{\prime}$. The cross-talk estimation will operate as

$$
\begin{aligned}
& \tilde{\boldsymbol{\alpha}}_{\mathbf{1}}=\operatorname{pinv}\left(\left[\begin{array}{ll}
\boldsymbol{x}_{1} & \boldsymbol{B}_{x_{2}}
\end{array}\right]\right) \boldsymbol{u}_{1}^{\prime}, \quad \tilde{\boldsymbol{\alpha}}_{\mathbf{2}}=\operatorname{pinv}\left(\left[\begin{array}{ll}
\tilde{\boldsymbol{x}}_{2} & \tilde{\boldsymbol{B}}_{x_{1}}
\end{array}\right]\right) \tilde{\boldsymbol{y}}_{2} \\
& \hat{\boldsymbol{\alpha}}_{1}=\tilde{\boldsymbol{\alpha}}_{1}(2: M+1), \quad \hat{\boldsymbol{\alpha}}_{2}=\tilde{\boldsymbol{\alpha}}_{2}(2: M+1)
\end{aligned}
$$

The resulting perturbation from the memory terms will degrade the cross-talk estimation. To study the effect of the relative strength and the number of the memory taps on cross-talk estimation, we consider 4 cases with different memory profiles named as a, b, c and d.

a) $\boldsymbol{m}_{1}=\boldsymbol{m}_{2}=\left[\begin{array}{lllllllll}0.1 & 0 & 0 & 0 & 0 & 0 & 0 & 0 & 0\end{array}\right]$,

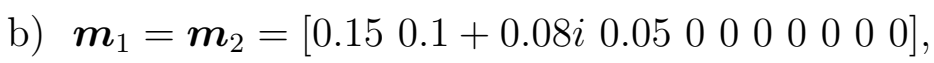

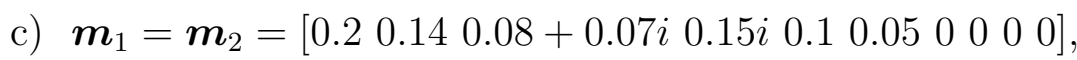

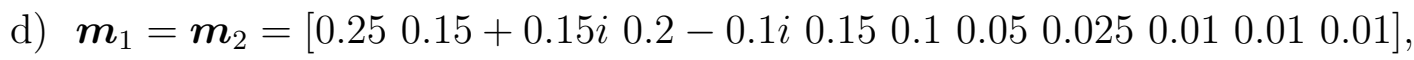

where $\boldsymbol{m}_{1}=\left[m_{1}(1) m_{1}(2) \ldots m_{1}(D)\right]$ and $\boldsymbol{m}_{2}$ is also defined similarly. Note that we model the memory effect in the linear section of the PA with these profiles. The average error, $\varepsilon_{\alpha}$ in Fig. C.3 shows that the quality of cross-talk estimation degrades 
as the memory effect in the linear section of the PA becomes stronger. From Fig. C.1 and Fig. C.3, it can be seen that the average errors in cross-talk estimation from the memory effect in the measurement results which are discussed in chapter 3 , are lesser than that of the memory profile $b$. 



\section{Bibliography}

[1] A. Paulraj, D. Gore, R. Nabar, and H. Bolcskei, "An overview of MIMO communications: A key to gigabit wireless," Proceedings of the IEEE, vol. 92, no. 2, pp. 198-218, Feb. 2004.

[2] "Spectral efficiency comparison table - wikipedia, the free encyclopedia." [Online]. Available: http://en.wikipedia.org/wiki/Template: Spectral_efficiency_comparison_table

[3] Prem Swaroop and Kelvin. G. Gard, "Crest factor reduction through in-band and out-of-band distortion optimizationl," IEEE Radio and wireless symposium., vol. AC-11, pp. 759-762, Jan. 2008.

[4] S. A. Bassam, M. Helaoui, S. Boumaiza, and F. M. Ghannouchi, "Experimental study of the effects of RF front-end imperfection on MIMO transmitter performance," in IEEE MTT-S International Microwave Symposium Digest. IEEE, 2008, pp. 1187-1190.

[5] F. F. D. Yin Shi, "MIMO RFIC transceiver designs for WLAN applications," in International Conference on ASIC, ASICON, 2007, pp. 348 - 351.

[6] Y. Palaskas, A. Ravi, S. Pellerano, B. R. Carlton, M. A. Elmala, R. Bishop, G. Banerjee, R. B. Nicholls, S. K. Ling, N. Dinur, S. S. Taylor, and K. Soumyanath, "A 5-GHz 108-mb/s 2x2 MIMO transceiver RFIC with fully integrated 20.5-dBm p1db power amplifiers in 90-nm CMOS," IEEE Journal of Solid-State Circuits, vol. 41, no. 12, pp. 2746-2756, Dec. 2006.

[7] W.-C. Hua, P.-T. Lin, C.-P. Lin, C.-Y. Lin, H.-L. Chang, C. W. Liu, T.-Y. Yang, and G.-K. Ma, "Coupling effects of dual SiGe power amplifiers for 802.11 n MIMO applications," in IEEE Radio Frequency Integrated Circuits (RFIC) Symposium, 2006, pp. 4-pp.

[8] S. Bassam, M. Helaoui, and F. Ghannouchi, "Crossover digital predistorter for the compensation of crosstalk and nonlinearity in MIMO transmitters," IEEE Transactions on Microwave Theory and Techniques, vol. 57, no. 5, pp. 11191128, May 2009.

[9] P. Suryasarman, M. Hoflehner, and A. Springer, "Digital pre-distortion for multiple antenna transmitters," in IEEE European Microwave Conference (EuMC), 2013, pp. 412-415.

[10] P. Suryasarman and A. Springer, "A comparison of cross-over and cross-talk cancelling digital predistorters for multiple antenna transmitters," Submitted to IEEE Transactions on Microwave Theory and Techniques, 2014. 
[11] M. V. Amiri, M. Helaoui, and F. M. Ghannouchi, "Streamlined MIMO crossover digital predistortion," in IEEE Radio and Wireless Symposium (RWS), 2014, pp. 283-285.

[12] P. Suryasarman and A. Springer, "Adaptive digital pre-distortion for multiple antenna transmitters," in IEEE Global Conference on Signal and Information Processing (GlobalSIP), 2013, pp. 1146-1149.

[13] — - "A comparative analysis of adaptive digital predistortion algorithms for multiple antenna transmitters," Accepted for publication in IEEE Transactions on Circuits and systems I, 2014.

[14] M. M. A. Hossain and R. Jantti, "Impact of efficient power amplifiers in wireless access," in Online Conference on Green Communications (GreenCom), 2011 IEEE. IEEE, 2011, pp. 36-40.

[15] J. C. Pedro, N. B. Carvalho, C. Fager, and J. A. Garcia, "Linearity versus efficiency in mobile handset power amplifiers," Microwave Engineering Europe, 2004 .

[16] E. Aschbacher, "Digital pre-distortion of microwave power amplifiers," Ph.D. dissertation, Technical University, Vienna, 2005.

[17] F. H. Raab, P. Asbeck, S. Cripps, P. B. Kenington, Z. B. Popovic, N. Pothecary, J. F. Sevic, and N. O. Sokal, "Power amplifiers and transmitters for RF and microwave," IEEE Transactions on Microwave Theory and Techniques, vol. 50, no. 3, pp. 814-826, 2002.

[18] L. Ye, "Design and analysis of digitally modulated transmitters for efficiency enhancement," Ph.D. dissertation, 2013.

[19] R. Marsalek, "Contributions to the power amplifier linearization using digital baseband adaptive predistortion," Ph.D. dissertation, Universite de Marne la Valle, 2003.

[20] H. Li, D. Wang, Z. Chen, and N. Liu, "Behavioural modelling of power amplifiers with memory effects based on subband decomposition," IEEE Electronics Letters, vol. 43, no. 5, pp. 270-271, 2007.

[21] N. B. Carvalho and J. C. Pedro, "Two-tone IMD asymmetry in microwave power amplifiers," in IEEE MTT-S International Microwave Symposium Digest, vol. 1, 2000, pp. $445-448$.

[22] E. Ngoya, C. Quindroit, and J. Nebus, "On the continuous-time model for nonlinear-memory modeling of RF power amplifiers," IEEE Transactions on Microwave Theory and Techniques, vol. 57, no. 12, pp. 3278-3292, Dec. 2009.

[23] Kenney,J.S and Fedorenko, P., "Identification of RF power amplifier memory effect origins using third-order intermodulation distortion amplitude and phase asymmetry," in IEEE MTT-S International Microwave Symposium Digest, 2006, pp. 1121 - 1124 . 
[24] J. Pedro and S. Maas, "A comparative overview of microwave and wireless power-amplifier behavioral modeling approaches," IEEE Transactions on Microwave Theory and Techniques, vol. 53, no. 4, pp. 1150-1163, Apr. 2005.

[25] L. C. Nunes, P. M. Cabral, and J. C. Pedro, "A physical model of power amplifiers AM/AM and AM/PM distortions and their internal relationship," in IEEE MTT-S International Microwave Symposium Digest, 2013, pp. 1-4.

[26] Hummels, D. R. and Gitchell R. D., "Equivalent low-pass representations for bandpass volterra systems," IEEE Transactions on Communications, vol. Com 28, no. 1, pp. 140-142, Jan. 1980.

[27] M. Schetzen, The Volterra and Wiener Theories of Nonlinear Systems. New York: Wiley, 1980.

[28] L. Ding, G. Zhou, D. Morgan, Z. Ma, J. Kenney, J. Kim, and C. Giardina, "A robust digital baseband predistorter constructed using memory polynomials," IEEE Transactions on Communications, vol. 52, no. 1, pp. 159-165, Jan. 2004.

[29] P. L. Gilabert, G. Montoro, and E. Bertran, "On the wiener and hammerstein models for power amplifier predistortion," in IEEE Asia-Pacific Microwave Conference (APMC), vol. 2, 2005.

[30] L. Ding, R. Raich, and G. T. Zhou, "A hammerstein predistortion linearization design based on the indirect learning architecture," in IEEE International Conference on Acoustics, Speech, and Signal Processing (ICASSP), vol. 3, 2002, pp. III-2689.

[31] Esref Eskinat, Stanley H. Johnson, and William L. Luyben, "Use of hammerstein models in identification of nonlinear systems," AIChE Journal, vol. 37, no. 2, pp. 255-268, Feb. 1991.

[32] J. Kim and K. Konstantinou, "Digital predistortion of wideband signals based on power amplifier model with memory," IEEE Electronics Letters, vol. 37, no. 23, pp. 1417-1418, 2001.

[33] Hyunchul Ku and J. Kenney, "Behavioral modeling of nonlinear RF power amplifiers considering memory effects," IEEE Transactions on Microwave Theory and Techniques, vol. 51, no. 12, pp. 2495-2504, Dec. 2003.

[34] L. Ding, "Digital predistortion of power amplifiers for wireless applications," Ph.D. dissertation, Georgia Institute of Technology, 2004.

[35] Bai, E. W., "An optimal two stage identification al-gorithm for hammersteinwiener nonlinear systems," in American Control Conference, Jun. 1998, pp. $2756-2760$.

[36] A. Hagenblad,, "Aspects of the identification of wiener models," Ph.D. dissertation, Linkoping university, Sweden, 1999.

[37] Narendra K. S and Gallman P. G, "An iterative method for the identification of nonlinear systems using a hammerstein model," IEEE Trans.Automat. Contr., vol. AC-11, pp. 546-550, Jul. 1966. 
[38] S. C. Cripps, RF power amplifiers for wireless communications. Boston: Artech House, 2006. [Online]. Available: http://site.ebrary.com/id/10160974

[39] M. Hoflehner and A. Springer, "Comparison of RF power amplifier behavioral models with respect to their modeling capabilities in adjacent and alternate bands," in Computer Aided Systems Theory-EUROCAST, 2012, pp. 9-16.

[40] P. Kenington, RF and baseband techniques for software defined radio. London: Artech House, 2005.

[41] H. Paaso and A. Mammela, "Comparison of direct learning and indirect learning predistortion architectures," in IEEE International Symposium on Wireless Communication Systems, 2008, pp. 309-313.

[42] M. Schetzen, "Theory of pth-order inverses of nonlinear systems," IEEE Transactions on Circuits and Systems, vol. 23, no. 5, pp. 285-291, 1976.

[43] C. Eun and E. J. Powers, "A new volterra predistorter based on the indirect learning architecture," IEEE Transactions on Signal Processing, vol. 45, no. 1, pp. 223-227, 1997.

[44] M. Kumar, Y. Tan, and J. K. Sin, "Excellent cross-talk isolation, high-q inductors, and reduced self-heating in a TFSOI technology for system-on-a-chip applications," IEEE Transactions on Electron Devices, vol. 49, no. 4, pp. 584$589,2002$.

[45] Kim, C.S and Park, P, "Deep trench guard technology to suppress coupling between inductors in silicon RF ICs," in IEEE MTT-S International Microwave Symposium Digest, Jun. 2001, pp. 1873-1876.

[46] H.-S. Kim, K. A. Jenkins, and Y.-H. Xie, "Effective crosstalk isolation through $\mathrm{p} / \mathrm{sup}+/$ si substrates with semi-insulating porous si," IEEE Electronic Device Letters, vol. 23, no. 3, pp. 160-162, 2002.

[47] Y. H. Wu, A. Chin, K. H. Shih, C. C. Wu, C. P. Liao, S. C. Pai, and C. C. Chi, "Fabrication of very high resistivity si with low loss and cross talk," IEEE Electronic Device Letters, vol. 21, no. 9, pp. 442-444, 2000.

[48] C. Bai, "Patent US20120313700 - predistorter for a multi-antenna transmitter," Dec. 2012.

[49] S. Amin, P. N. Landin, P. Händel, and D. Ronnow, "Behavioral modeling and linearization of crosstalk and memory effects in RF MIMO transmitters," IEEE Transactions on Microwave Theory and Techniques, vol. 62, no. 4, pp. 810-823, Apr. 2014.

[50] R. D. DeGroat and E. M. Dowling, "The data least squares problem and channel equalization," IEEE Transactions on Signal Processing, vol. 41, no. 1, p. 407, 1993.

[51] D. Saffar, N. Boulejfen, F. M. Ghannouchi, A. Gharsallah, and M. Helaoui, "Behavioral modeling of MIMO nonlinear systems with multivariable polynomials," 
IEEE Transactions on Microwave Theory and Techniques, vol. 59, no. 11, pp. 2994-3003, Nov. 2011.

[52] M. A. Hussein and O. Venard, "Subband digital predistorsion based on indirect learning architecture," in IEEE International Conference on Acoustics, Speech and Signal Processing (ICASSP), 2014, pp. 7974-7978.

[53] A. Zhu, P. Draxler, J. Yan, T. Brazil, D. Kimball, and P. Asbeck, "Open-loop digital predistorter for $\mathrm{RF}$ power amplifiers using dynamic deviation reductionbased volterra series," IEEE Transactions on Microwave Theory and Techniques, vol. 56, no. 7, pp. 1524-1534, Jul. 2008.

[54] 3GPP-TS:25.213, "3rd generation partnership project TS 25.213, technical specification group radio access network; spreading and modulation (FDD)," 2004. [Online]. Available: www.3gpp.org

[55] 3GPP-TS:36.211, "3rd generation partnership project TS 36.211, technical specification group radio access network; evolved universal terrestrial radio access (e-UTRA); physical channels and modulation," 2007. [Online]. Available: http://www.3gpp.org/

[56] P. Landin, M. Isaksson, and P. Händel, "Comparison of evaluation criteria for power amplifier behavioral modeling," in IEEE MTT-S International Microwave Symposium Digest, 2008, 2008, pp. 1441-1444.

[57] 3GPP-TS:25.141, "3rd generation partnership project; TS 25.141, technical specification group radio access network; base station conformance testing (FDD)," 2003. [Online]. Available: http://www.3gpp.org/

[58] 3GPP-TS:36.101, "3rd generation partnership project; TS 36.101, technical specification group radio access network; evolved universal terrestrial radio access (e-UTRA); user equipment (UE) radio transmission and reception (release 8)." [Online]. Available: http://www.3gpp.org/

[59] O. Hammi, S. Boumaiza, B. Vassilakis, and F. M. Ghannouchi, "On the effects of the average power of training sequences used to synthesize memory digital predistorters in wcdma transmitters," in in Proc. European Microwave Conference., 2007.

[60] P. Suryasarman, L. Peng, and A. Springer, "Optimizing the identification of digital predistorters for improved power amplifier linearization performance," IEEE Transactions on Circuits and Systems II, vol. 61, no. 9, pp. 671-675, Sep. 2014.

[61] J. K. Cavers, "Amplifier linearization using a digital predistorter with fast adaptation and low memory requirements," IEEE Transactions on Vehicular Technology, vol. 39, no. 4, pp. 374-382, 1990.

[62] R. Santucci and A. Spanias, "A block adaptive predistortion algorithm for transceivers with long transmit-receive latency," in IEEE International Sympo- 
sium on Communications, Control and Signal Processing (ISCCSP), 2010, pp. $1-6$.

[63] F. Gregorio, J. Cousseau, S. Werner, T. Riihonen, and R. Wichman, "Power amplifier linearization technique with IQ imbalance and crosstalk compensation for broadband MIMO-OFDM transmitters," EURASIP Journal on Advances in Signal Processing, vol. 2011, no. 1, pp. 1-15, 2011.

[64] N. Zheng and C. Yuelin, "Digital predistortion based on qrd-rls algorithm and its implementation using fpga," in 1st International Conference on Information Science and Engineering (ICISE). IEEE, 2009, pp. 200-203.

[65] L. You and Z. Xiaolin, "Adaptive digital predistortion based on mc-fqrd-rls algorithm using indirect learning architecture," in International Conference on Advanced Computer Control (ICACC). IEEE, 2010, pp. 240-242.

[66] S. Mushtaq and M. John, "Qr-decomposition based algorithms for adaptive volterra filtering," IEEE Transactions on circuits and systems 1, vol. 40, no. 6, pp. 372-382, 1993.

[67] J. Wei and Y. Chongxiu, "Ofdm adaptive digital predistortion method combines rls and lms algorithm," in 4th IEEE Conference on Industrial Electronics and Applications, ICIEA ., 2009, pp. 3900-3903.

[68] H. Garcia and G. Prieto, "Digital predistorter based on volterra series for nonlinear power amplifier applied to ofdm systems using adaptive algorithms," in International Meeting of Electrical Engineering Research, 2012, pp. 118-125.

[69] S. Haykin, Adaptive Filter Theory, 4th ed. Pearson Education, 2007.

[70] M. E. Hoff and B. Widrow, "Adaptive switching circuits," WESCON Conv. Rec, vol. 4, pp. 96-104, Aug. 1960.

[71] R. L. Plackett, "Some theorems in least squares," Biometrika, vol. 37, no. 1/2, p. 149, Jun. 1950.

[72] H. A. Jan Leeuwen, "The complexity of basic complex operations," University of Utrecht, Netherlands, Technical Report RUU-CS-79-4, Jun. 1979.

[73] Gaussian-elimination, "Wikipedia, the free encyclopedia." [Online]. Available: http://en.wikipedia.org/wiki/Gaussian_elimination

[74] Peter Danziger, "Complexity of the gaussian algorithm." [Online]. Available: http://www.math.ryerson.ca/ danziger/professor/MTH108/Handouts/ gauss-complexity.pdf

[75] L. D. Zhengxiang Ma, "Patent US20030223508 - system and method for predistorting a signal using current and past signal," May 2002.

[76] L. Lu, L. Geoffrey, and S. Lee, "An overview of massive mimo:benefits and challenges," IEEE Journal Of Selected Topics In Signal Processing, vol. 8, no. 5, pp. 742-758, Oct. 2014. 


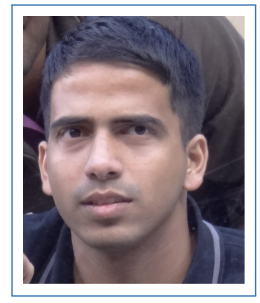

Padmanabhan Suryasarman

Curriculum Vitae

\title{
Summary
}

○ 8 years of industrial and research experience (including M.Tech and Doctoral studies) in the area of Wireless Communication, Signal Processing, and RF-systems.

- Co-author of 7 international peer-reviewed publications and 4 US patents.

\section{Education}

2011-2015 Doktor der Technischen Wissenschaften (Dr.techn.), Johannes (Expected) Kepler University, Linz, Austria.

Currently working towards the doctoral degree at the Institute for Communications Engineering and RF-Systems (NTHFS). Expecting to complete in April 2015.

2006-2008 Master of Technology (M.Tech), Indian Institute of Technology Madras, Chennai, India.

M.Tech degree in Communication Engineering from the Electrical Engineering Dept, CGPA: 8.94/10. Major project: Channel Estimation and Tracking for IEEE 802.16e standard, and MIMO MMSE DFE for SC-FDMA, with Prof. K.Giridhar.

2001-2005 Bachelor of Technology (B.Tech), National Institute of Technology, Calicut, India.

B.Tech in Electronics and Communication Engineering, GPA: $70.8 \%$

\section{Doctoral Thesis}

Title Digital pre-distortion for multiple antenna transmitters.

Supervisor Prof. Andreas Springer

\author{
Altenberger Str 69 - 4040 Linz - Austria \\ (8) $+43(0) 68120527528 \bullet \mathbf{0}+43(0) 73224686411$ \\ FAX $+43(0) 73224686374$ \\ $\bowtie$ padmanabhan.ms@gmail.com,p.madampu@nthfs.jku.at
}


Description RF cross-talk before the Power amplifiers (PA) in multiple antenna transmitters (MIMO Tx) adversely affects the performance of digital predistortion (DPD). A cross-talk canceling DPD (CTC-DPD) is proposed, which performs better and computationally simpler compared to the state-of-the-art Cross-over DPD (CO-DPD).

\title{
Experience
}

2011-Present Student Employee, System Engineering, Danube Mobile Commu(3 years and nications Engineering (Intel, Linz), Linz, Austria.

8 months) Worked in the Uplink Tx MIMO team, on several issues regarding MIMO and carrier aggregation for HSUPA and 3GPP-LTE, such as remodulation, intermodulation, RF-leakage etc. Worked in the SACRA project in the development of a flexible RF transceiver front-end that supports MIMO and carrier aggregation.

2008-2011 Reasearch Engineer, Centre of Excellence in Wireless Technology

(2 years and (CEWiT), Chennai, India.

8 months) The reaserch focus was on enhancing the cell-edge coverage and increasing the data rate for cell-edge users in the future broadband wireless systems such as IEEE $802.16 \mathrm{~m}$ and 3GPP-LTE. Worked in the development of link-level and system level simulators for $802.16 \mathrm{~m}$ and LTE. Studied the performance of various MIMO schemes, receiver combining (especially MMSE-IRC) algorithms and pilot design. Worked on Conjugate Data Repetition (CDR) and Collision Free Interlaced Pilots (CoFIP). IEEE 802.16m standard has adopted CDR and CoFIP. Attended several IEEE $802.16 \mathrm{~m}$ standardization meetings in the Republic of Korea and the United States.

2005-2006 Software Engineer, IBM, Bengaluru, India.

(1 year) Worked in IBM Mainframe systems and DEC/EDI.

Computer skills

OS Linux, Unix, Windows.

Programming $\mathrm{C} / \mathrm{C}++/ \mathrm{IT}++$, Matlab,VHDL.

\section{Interests}

Signal processing for communication systems, Wireless communications, MIMO technology, Software defined radio and Power amplifier predistortion.

\section{Publications}

[1] P. Suryasarman and A. Springer, "A Comparative Analysis of Adaptive Digital Predistortion Algorithms for Multiple Antenna Transmitters," Accepted for publication in IEEE Trans. on Circuits systems I, 2015.

\author{
Altenberger Str 69 - 4040 Linz - Austria \\ (8) $+43(0) 68120527528 \bullet \mathbf{0}+43(0) 73224686411$ \\ FAX $+43(0) 73224686374$ \\ 凶padmanabhan.ms@gmail.com,p.madampu@nthfs.jku.at
}


[2] P. Suryasarman and A. Springer, "A Comparison of Cross-over and Crosstalk Cancelling Digital Predistorters for Multiple Antenna Transmitters," Submitted to IEEE Trans. on Microwave Theory and Techniques, 2015.

[3] P. Suryasarman, P. Liu, and A. Springer, "Optimizing the identification of digital predistorters for improved power amplifier linearization performance," IEEE Trans. on Circuits and systems II, Exp. Brief, early access, 2014.

[4] P. Suryasarman and A. Springer, "Adaptive digital pre-distortion for multiple antenna transmitters," in IEEE Global Conference on Signal and Information Processing (GlobalSIP) 2013, pp. 1146-1149.

[5] P. Suryasarman, M. Hoflehner, and A. Springer, "Digital pre-distortion for multiple antenna transmitters," in IEEE European Microwave Conference (EuMC), 2013, p. 412-415.

[6] P. Karunakaran, P.Suryasarman, V.Ramaswamy, K.Kuchi, J.K.Milleth, B.Ramamurthi, "On Pilot Design for Interference Limited OFDM Systems", International Symposium on Wireless Communication Systems, Nov 2011.

[7] K.Kuchi, R.Vinod, M.K.Dileep, M.S.Padmanabhan, R.Dhivagar, "Interference Mitigation Using Conjugate Data Repetition", International Conference on Communication, Jun 2009.

[8] M.S.Padmanabhan, R.Vinod, K.Kuchi, K.Giridhar "MMSE DFE for MIMO DFT spread OFDMA", National Conference on Communication, Jan 2009.

\section{Patents}

[1] K. Kuchi, D. Jeniston, V. Ramaswamy, B. Dhivagar, K. Giridhar, B. Ramamurthi, D. Kalathil, P. Suryasarman, "Precoding for Multiple Transmission Streams in Multiple Antenna Systems", US patent, US8699446 B2, 2014.

[2] B. Dhivagar, G. George, K. Giridhar, D. Kalathil, P. Karunakaran, J. Milleth, K. Kuchi, B. Ramamurthi, V. Ramaswamy, Y. Reddy, P. Suryasarman, "Pilot Aided Data Transmission and Reception with Interference Mitigation in Wireless Systems", US Patent App, 13/254,849, 2010 .

[3] K. Kuchi, D. Jeniston, V. Ramaswamy, B. Dhivagar, K. Giridhar, B. Ramamurthi, D. Kalathil, P. Suryasarman, "Precoding for Single Transmission Streams in Multiple Antenna Systems", US Patent App, 12/999,894, 2009 .

[4] B. Dhivagar, K. Giridhar, D. Jeniston, D. Kalathil, K. Kuchi, B. Ramamurthi, V. Ramaswamy, P. Suryasarman, "Methods and Systems for Interference Mitigation", US Patent App, 12/999,826, 2009.

$$
\begin{gathered}
\text { Altenberger Str 69-4040 Linz - Austria } \\
+43(0) 68120527528 \bullet \mathbf{0}+43(0) 73224686411 \\
\text { [FAX +43(0)73224686374 } \\
\text { 凶padmanabhan.ms@gmail.com,p.madampu@nthfs.jku.at }
\end{gathered}
$$

